\title{
A CRITICAL EXPLORATION OF PROFESSIONAL PERCEPTIONS OF HARM REDUCTION POLICY AND PRACTICE IN VIETNAM
}

\author{
By
}

TUAN DUNG TRUONG

A thesis submitted to Victoria University of Wellington in fulfilment of the requirements for the degree of Doctor of Philosophy

Victoria University of Wellington

2019 



\begin{abstract}
Throughout the 1990s, Vietnam experienced a dramatic rise in the prevalence of HIV among people who use drugs. In response, Vietnam's Ministry of Health implemented several legal and policy interventions in the name of harm reduction. However, perceptions about drug use, people who use drugs, addiction and the nature of official interventions are contested. For many Vietnamese officials, abstinence remains the dominant philosophy. Drug use is considered a 'social evil' in Vietnam and people who use drugs face draconian controls and incarceration in the name of treatment and crime prevention.
\end{abstract}

Against this background, this thesis explores how key stakeholders perceive harm reduction philosophy and how they apply it in policy and practice. Based on qualitative and quantitative methods, it presents findings from a survey with 250 respondents and 26 semi-structured interviews, all with professionals involved in responding to drug use in Vietnam. The thesis illustrates that these professionals prefer abstinence approaches, and often see addiction as the result of moral failings and brain diseases.

While some interventions in the name of harm reduction are accepted, they are firmly rooted within a narrow public health perspective. Professional misperceptions about the key principles and practices of 'authentic' harm reduction are widespread. Many professionals believe, for example, that harms can only be limited through reductions in the demand and supply of drugs, or that detaining people who use drugs in compulsory treatment centres is a form of harm reduction.

These rationales have resulted in continuing police crackdowns, and the use of 'pseudo' harm reduction strategies to control and punish people who use drugs. Meanwhile, there are limited official attempts to address problems experienced by people who use drugs, like social isolation, stigma, discrimination, human rights violations, or problems of community reintegration. In conclusion, while a harm reduction rhetoric is regularly employed in Vietnam, 'pseudo' harm reduction strategies are carried out. 


\section{Acknowledgements}

This study has been a long time in the making. Many people and organisations have generously helped me over four years in my PhD journey. First and foremost, I would like to express my respectful gratitude to my primary and second supervisors, Professor Elizabeth Stanley and Dr Fiona Hutton for their tremendous support and encouragement throughout my study process. I am especially indebted to my former supervisor, Associate Professor Julian Buchanan who has been an endless source of strength and inspiration that nurtures my dream of becoming a harm reductionist. Additionally, I would also like to express my profound gratitude to Kirsten and Deborah from Student Learning for their helpful feedback and invaluable recommendations during my study.

I would like to express particular thanks to the Ministry of Public Security, the 911 Vietnamese Project and Victoria University of Wellington for funding my PhD study. My appreciation also goes to the Faculty of Graduate Research for the financial support provided to me to do fieldwork and attend conferences. Thanks also must go to Head of School Professor Brigitte Bonisch-Brednich, administration staff and the Student Learning team for their enthusiastic support throughout my studies.

I would like to thank all the study participants, who gave up valuable time to be interviewed. Thanks also must go to my colleagues at Police College and my leaders, Professor Khien, $\mathrm{Mr}$ Khoi, Mr Tao and Dr Em, for tremendous support and useful assistance. Thanks to my friends, Brent, Max, Sarah and Reena, who share the same PhD endeavour, for their psychological support and encouragement. I particularly thank my friend, Hoe, for his inspiration that fosters me to research harm reduction in Vietnam. Also, I would like to thank other friends, Dr Hai, Dr Thu Vuong and Dr Tinh for their academic support. I would like to thank Madeleine for proofreading my thesis.

Last, but not least, this PhD is sincerely dedicated to my parents, my brother and other family members. Their wholehearted support encouraged me to pursue my goal and accomplish this thesis. 


\section{Table of Contents}

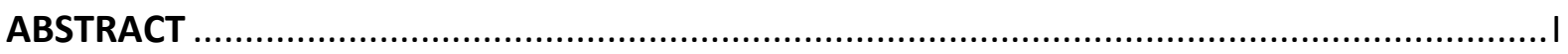

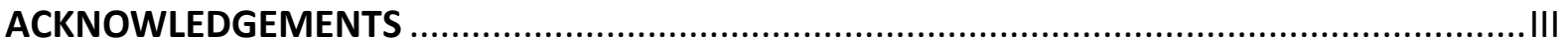

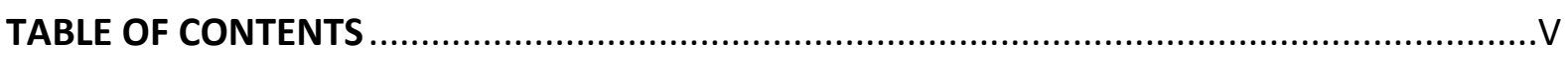

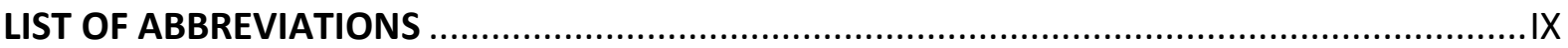

LIST OF TABLES

LIST OF FIGURES

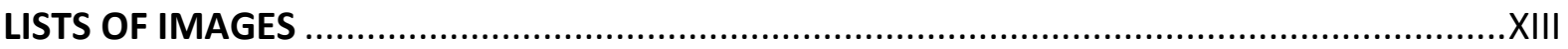

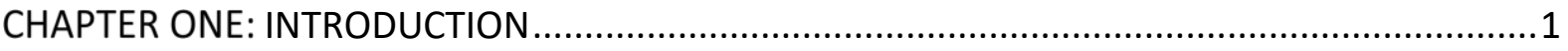

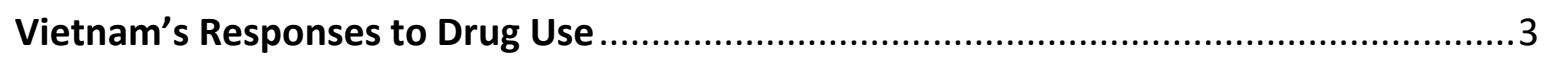

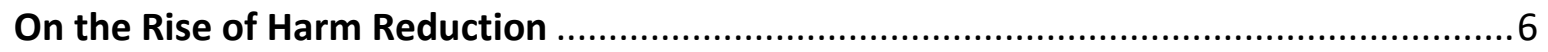

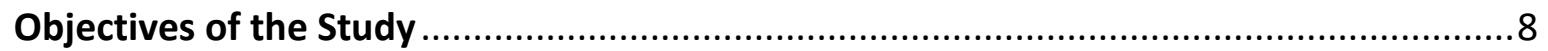

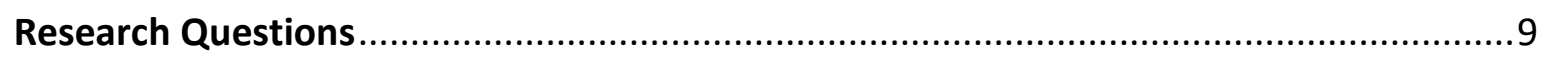

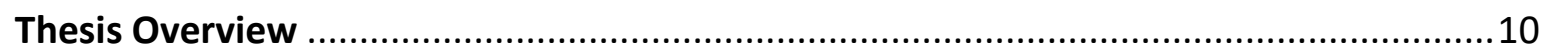

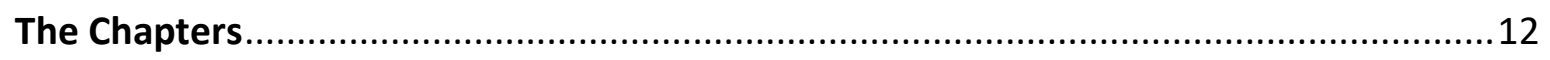

CHAPTER TWO: ABSTINENCE: A GLOBAL RESPONSE TO DRUG USE ..................................17

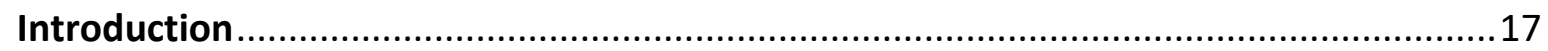

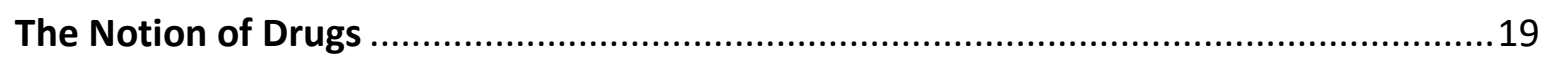

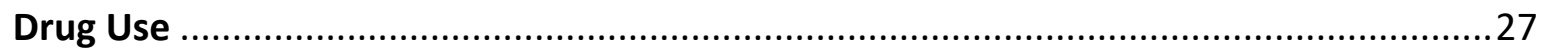

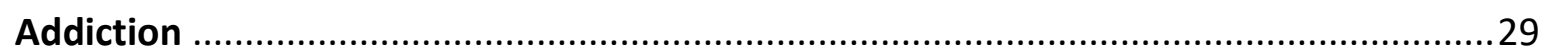

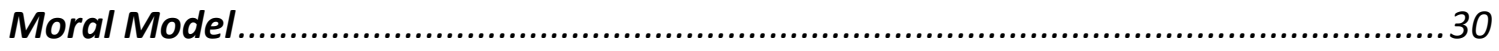

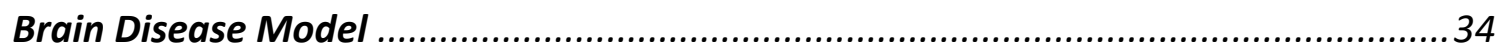

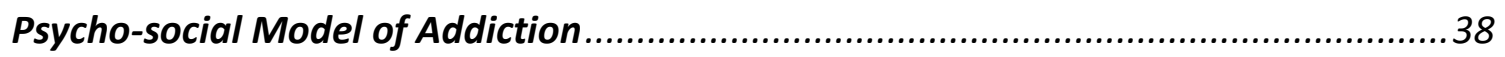

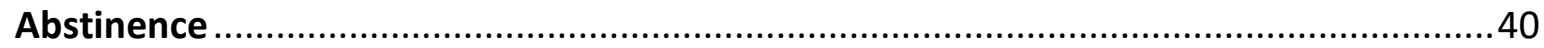

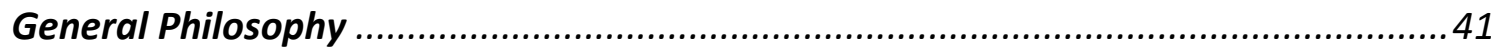

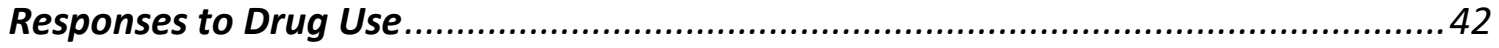

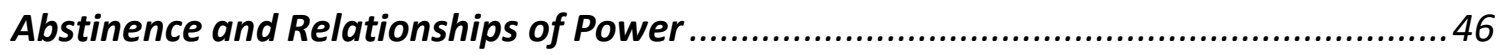

Counterproductive Effects of Enforced Abstinence ...................................................4 47

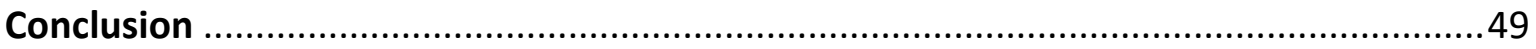

CHAPTER THREE: FROM ABSTINENCE TO HARM REDUCTION ............................................51

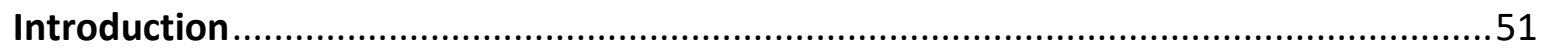

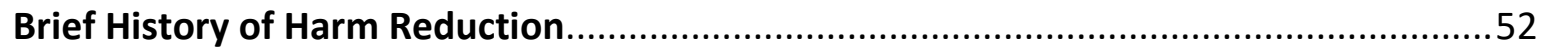

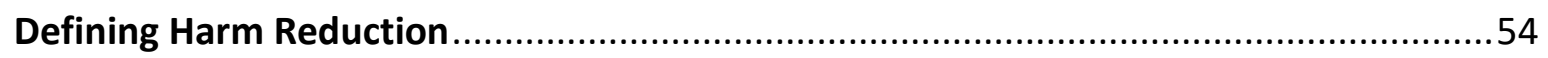

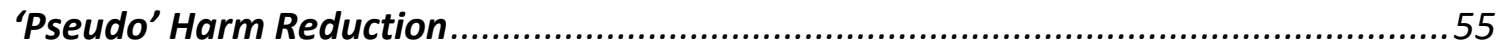

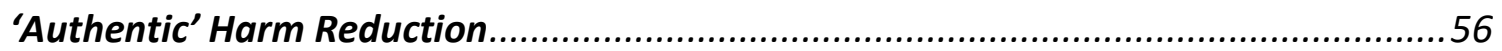

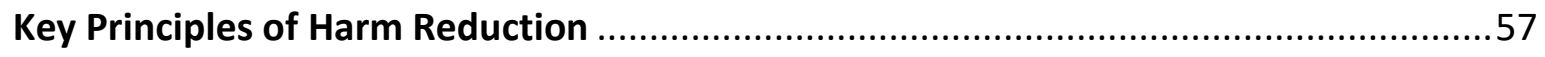




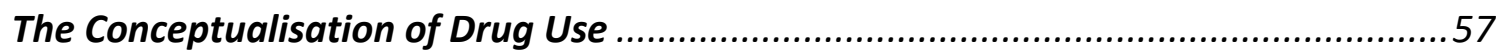

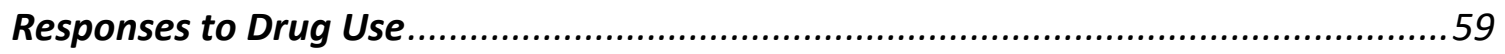

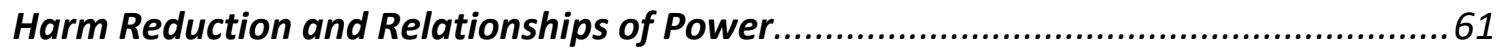

Harm Reduction, Human Rights and Drug Policy Reform ...........................................62

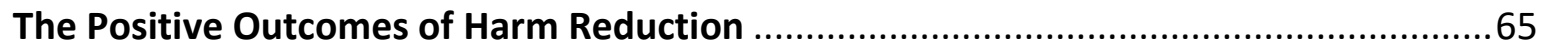

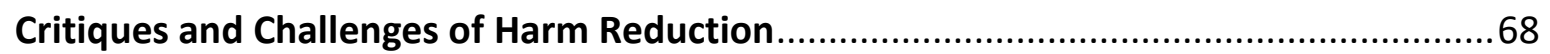

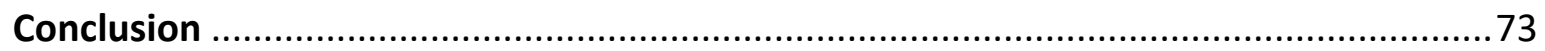

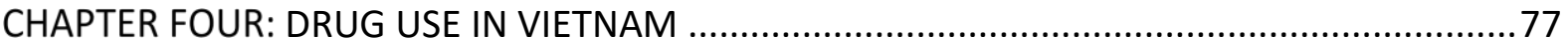

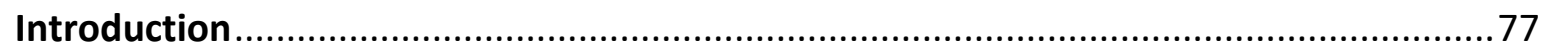

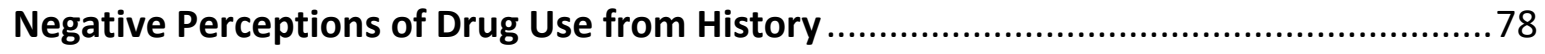

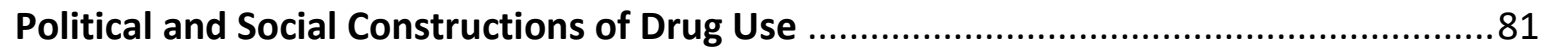

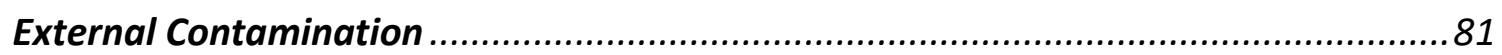

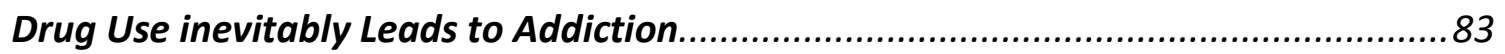

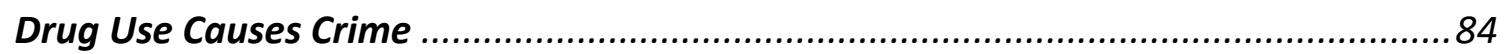

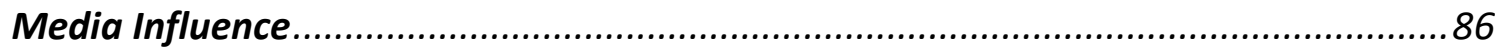

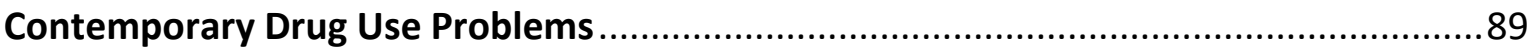

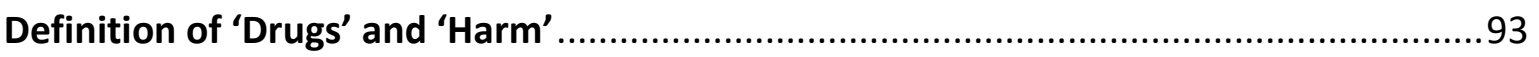

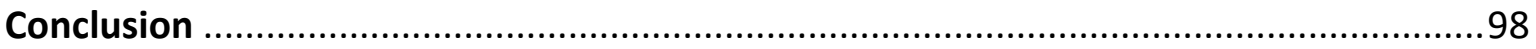

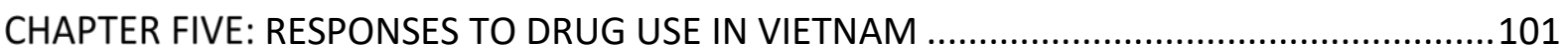

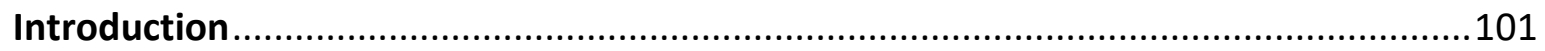

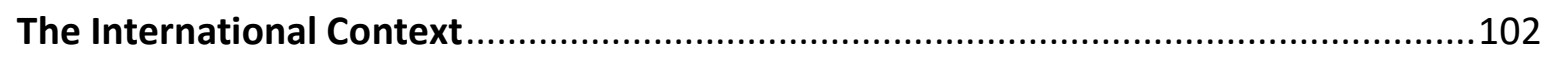

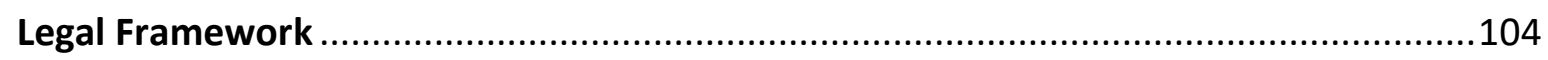

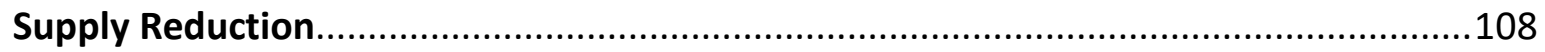

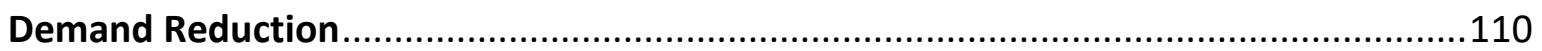

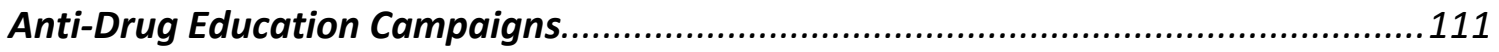

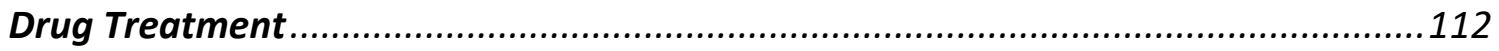

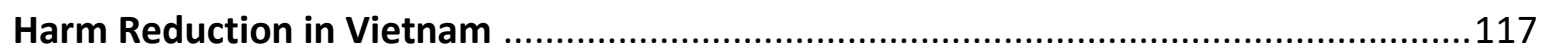

A Chronology of Harm Reduction in Vietnam ..................................................... 118

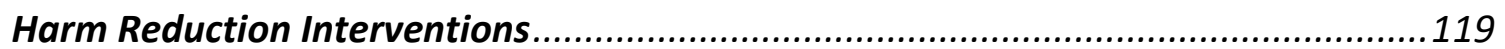

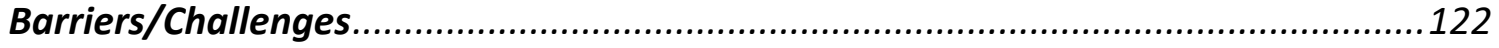

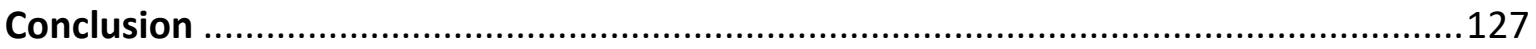

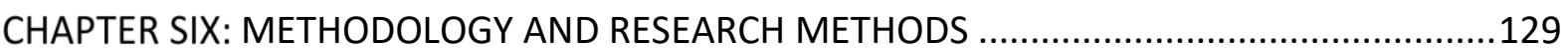

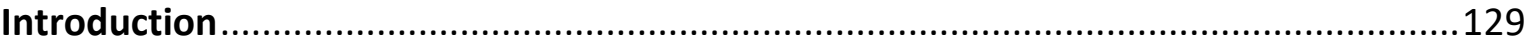

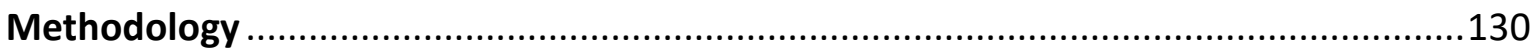

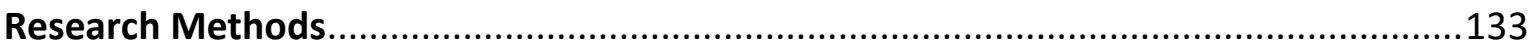

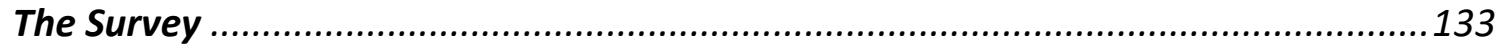

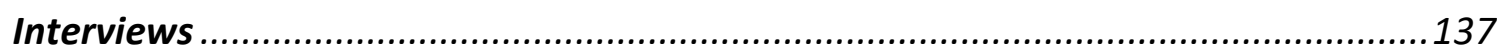

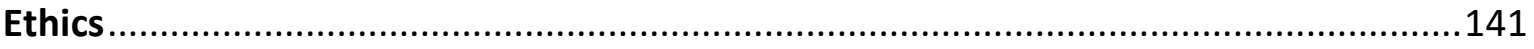

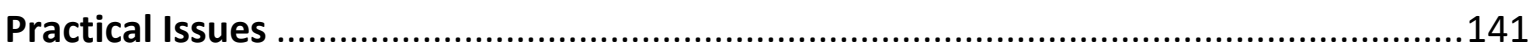

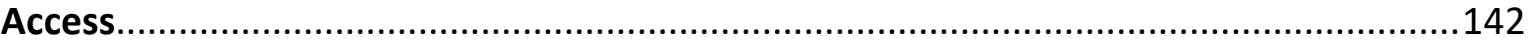

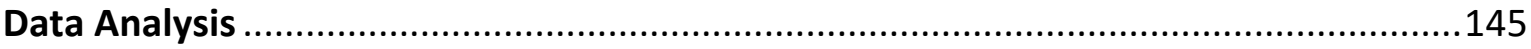

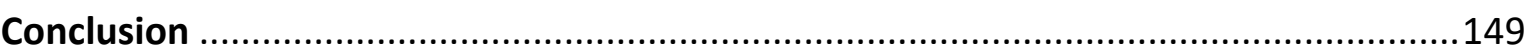




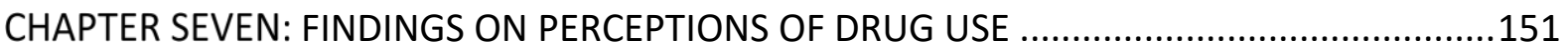

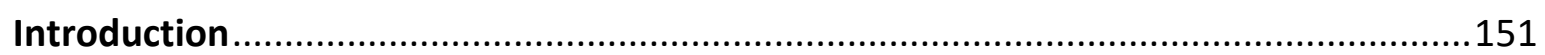

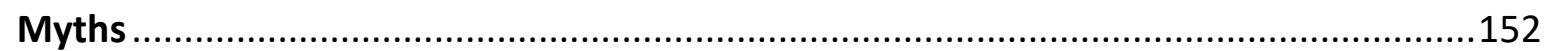

Myth One: Illicit Drugs Are the Most Harmful ....................................................... 152

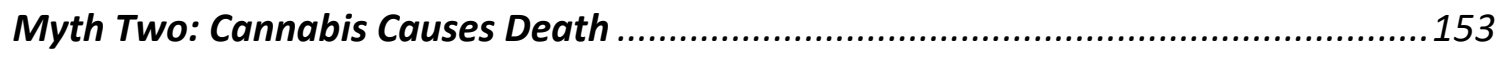

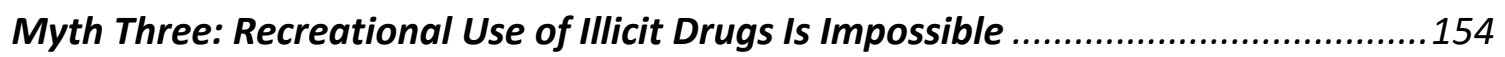

Myth Four: Illicit Drug Use Inevitably Leads to Addiction .........................................156

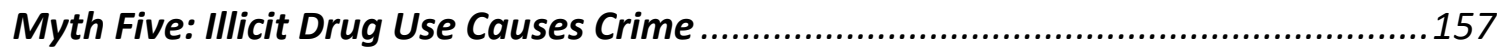

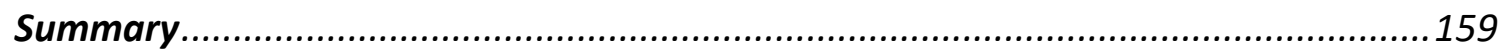

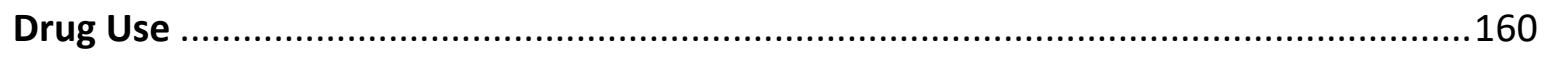

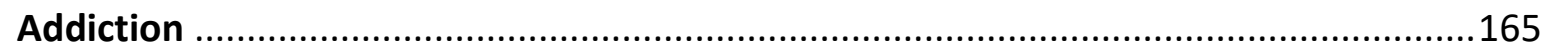

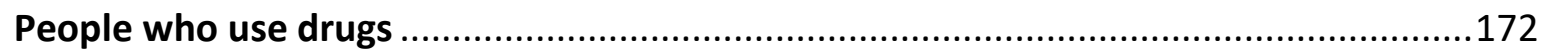

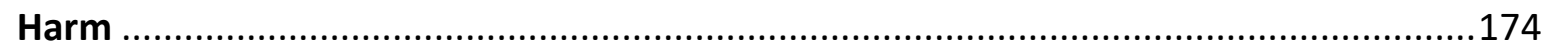

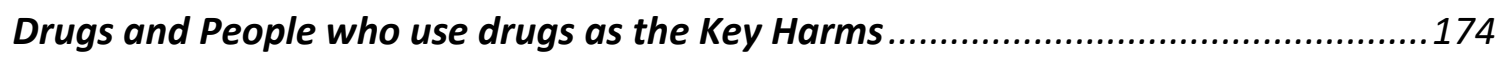

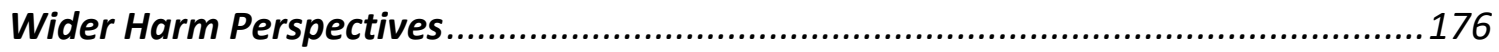

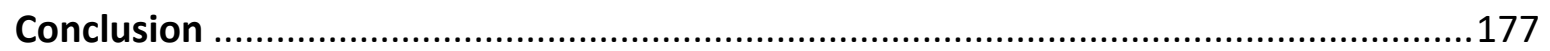

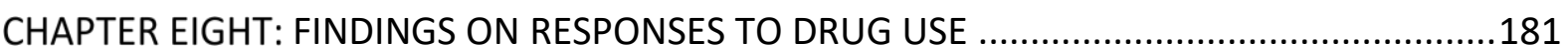

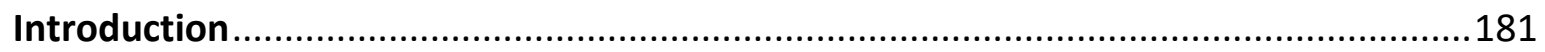

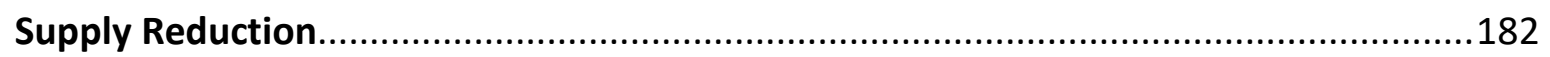

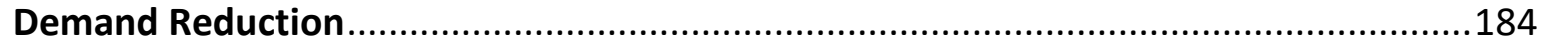

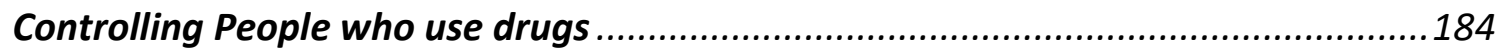

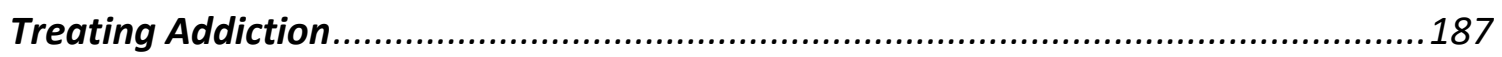

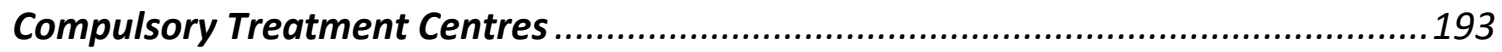

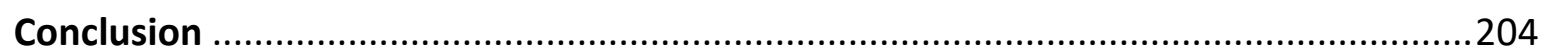

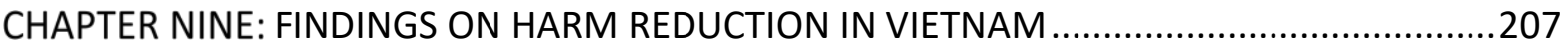

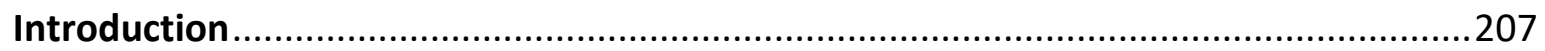

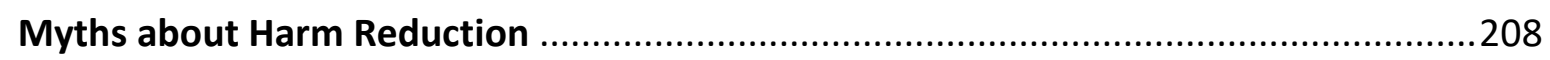

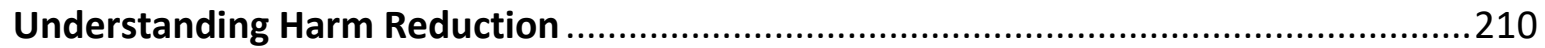

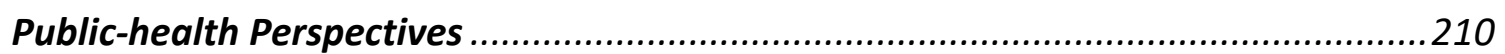

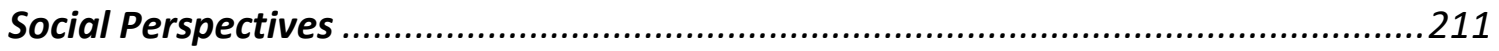

Attitudes towards Practical Interventions of Harm Reduction ...................................213

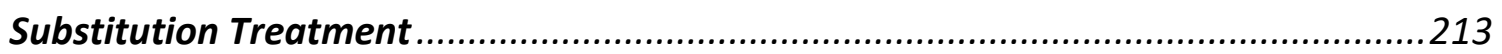

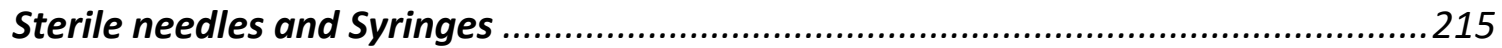

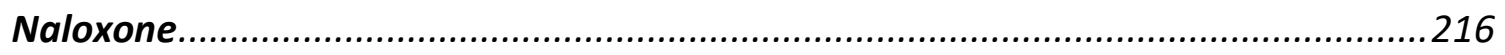

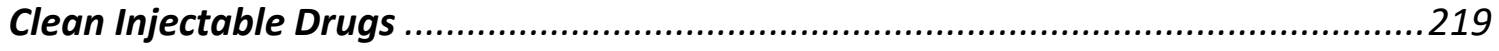

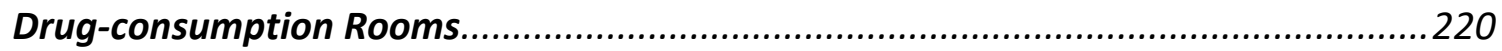

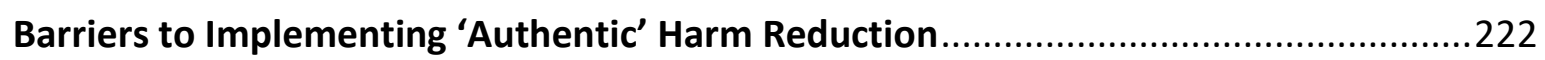

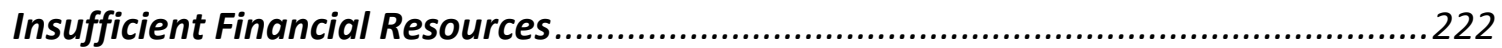

Limited Understanding of 'Authentic' Harm Reduction ...........................................224

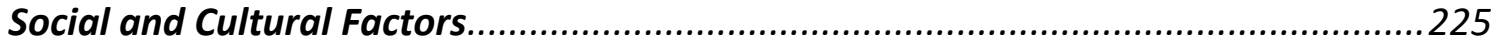

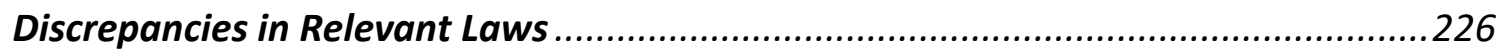

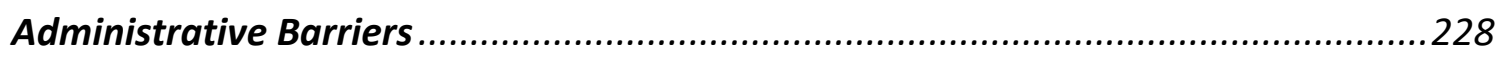

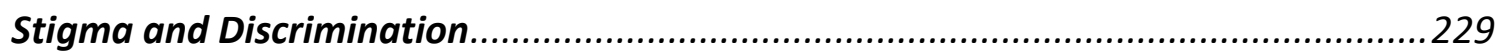




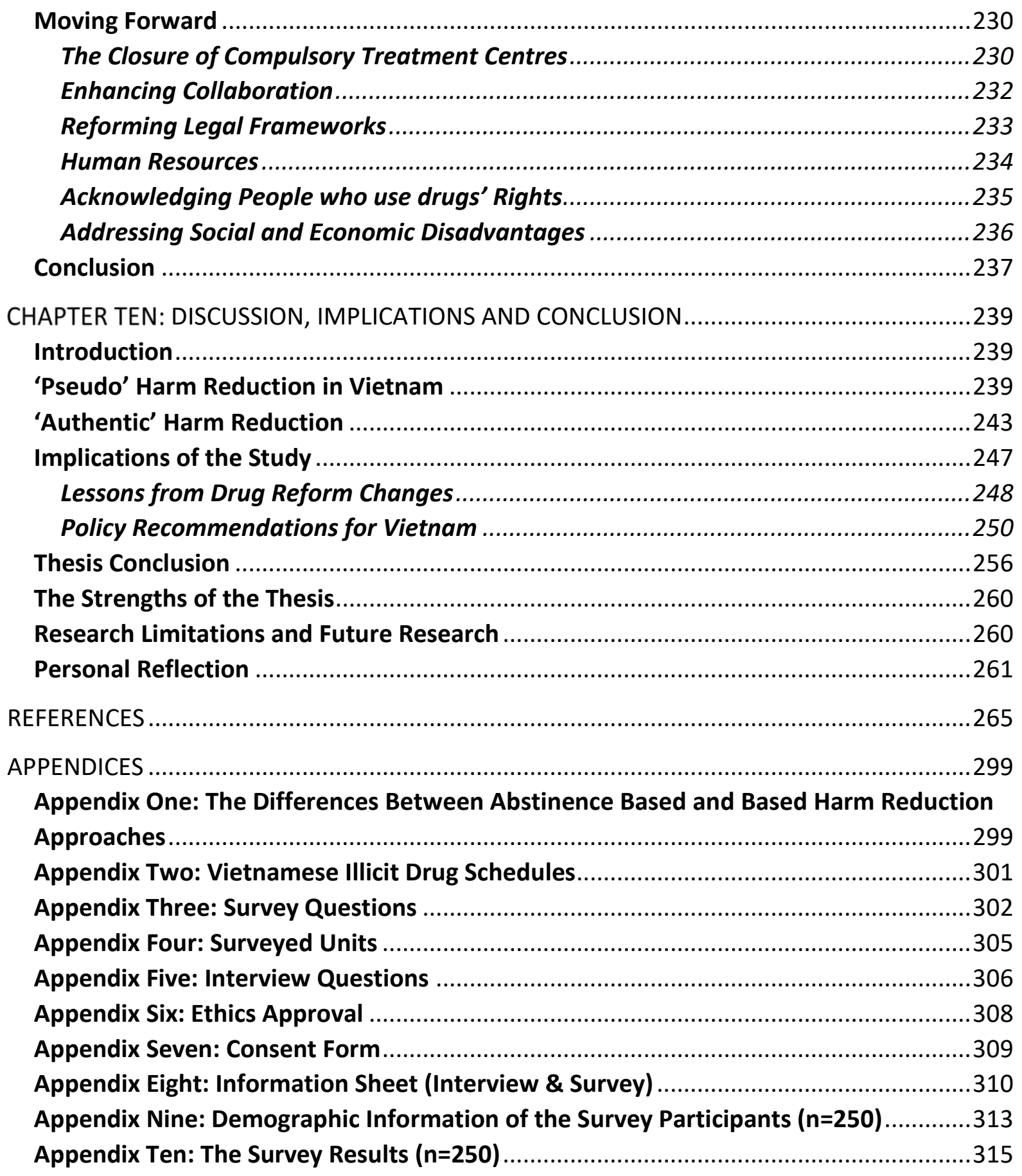




\section{List of Abbreviations}

ASEAN

ATS

ART

NSDPs

$\mathrm{MOH}$

MMT

MOLISA

MPS

NGOs

PWID

PWUD

UN

UNAIDS

UNODC

WB

WHO
The Association of Southeast Asian Nations

Amphetamine-type Stimulants

Antiretroviral treatment

Needles and Syringes Distribution Programmes

Ministry of Health

Methadone Maintenance Treatment

Ministry of Labour, War Invalids and Social Affairs

Ministry of Public Security

Non-Government Organisations

People who inject drugs

People who use drugs

United Nations

Joint United Nations Program on HIV/AIDS

United Nations Office on Drugs and Crime

World Bank

World Health Organisation 


\section{List of Tables}

Table 4.1: Trend in use of selected drugs in Vietnam, 2011-2015 (UNODC, 2017, p. 50) ......91

Table 4.2: Seizures of selected drugs in Vietnam, 2011-2015 (UNODC, 2017, p. 51)............91

Table 6.1: The work setting, gender and work location of survey respondents $(n=250) \ldots . . .136$

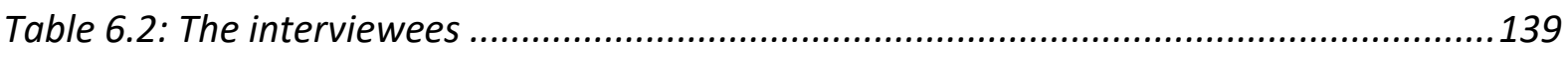

Table 6.3: The initial and changed questions on compulsory treatment centres.................145 


\section{List of Figures}

Figure 4.1: Drugs ordered by their overall harm scores (Nutt et al., 2010; Nutt, King,

Saulsbury, \& Blakemore, 2007) 96

Figure 5.1 The development of Vietnam's drug policies. 107

Figure 5.2 Estimated cultivation of opium (1990 to 2016) (Luong, 2017; MPS, 2015)....... 109

Figure 5.3 The number of methadone patients in Vietnam (2008-2017) 121

Figure 7.1: Some illegal drugs are less harmful than alcohol and tobacco 153

Figure 7.2: Cannabis overdose deaths are a problem 154

Figure 7.3: Most people who take drugs do so recreationally and rationally 155

Figure 7.4: Taking drugs will inevitably cause drug dependence 157

Figure 7.5: Drug use is a crime problem 158

Figure 7.6: Drug use is a public health issue 161

Figure 7.7: Drug use is a normal human behaviour. 165

Figure 7.8: People who use drugs need to quit all drugs and stay drug-free..... 166

Figure 7.9: Drug relapse should be viewed as a learning experience 170

Figure 8.1: We can protect society by eradicating the supply of drugs. 183

Figure 8.2: We should create a society free from drugs 183

Figure 8.3: We can protect society by punishing drug possession 185

Figure 8.4: People who use drugs need to be forced to get treatment..... 187

Figure 8.5: People who use drugs need to quit all drugs and stay drug-free...... 190

Figure 8.6: Compulsory treatment is effective in preventing drug relapse 195

Figure 8.7: People who use drugs should play a central role in deciding their treatment goals

Figure 8.8: Abstaining from drugs is the only effective treatment for people who use drugs

Figure 9.1: Oral methadone is effective treatment for people who use drugs.... .214 
Figure 9.2: People who use drugs should be provided with sterile needles and syringes .....215

Figure 9.3: People who use drugs should be provided with naloxone (an opioid antidote) to

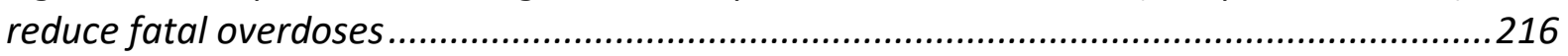

Figure 9.4: Medically supervised rooms (drug-consumption rooms) should be available ....221 


\section{Lists of Images}

Image 1.1 Drug flows to Vietnam and the rest of the Southeast Asia region from the Golden Triangle area (Luong, 2017).

Image 4.1 (Left): Young people say no to illicit drugs (The National Committee on AIDS Drugs and Prostitution Prevention and Control 2011).... .86

Image 4.2 (Right): Do not let drug use and HIV destroy your life (The National Committee on AIDS Drugs and Prostitution Prevention and Control 2011)

Image 5.1 (Left): Saving human's lives from narcotics (illicit drugs) (The National Committee on AIDS Drugs and Prostitution Prevention and Control 2011)

Image 5.2 (Right): Students say no to narcotics (The National Committee on AIDS Drugs and Prostitution Prevention and Control 2011). 


\section{Chapter One: Introduction}

Situated close to the 'Golden Triangle' region, and lying across important South-East Asian region traffic routes (See Image 1.1), Vietnam has a long history of producing and consuming drugs. The most common drug is opium, which was first introduced into Vietnam around the 1600s by migrant minorities from China (Luong, 2017), and consumed among ethnic minority groups in the northern mountainous areas. The level of opium production and consumption remained fairly limited, before demand and supply gradually increased during the $19^{\text {th }}$ and $20^{\text {th }}$ centuries, especially after the annexation of Vietnam by France in 1858 (Windle, 2012). Opium use then spread into other highland areas. In the early $20^{\text {th }}$ century, the newly installed colonial government promoted the production and sale of opium in Vietnam for financial purposes, and for population control (Edington \& Bayer, 2013). The negative attitudes among Vietnamese people towards drug use have emerged from the historical actions of colonial governments.

Drug use was first seen as a 'national enemy' because the French used opium as means to control, and even poison Vietnamese people (Rapin, Dao, and Pham (2003). Following Vietnam's independence in 1975 (after the first and second Indochina Wars ${ }^{1}$ ), the country had to deal with a great number of citizens who remained dependent on drugs (roughly 200,000 drug users) (Pham et al., 2010). Drug use was then seen as a leftover of the old regime and a form of external contamination from the west (Edington, 2016). Contemporary drug use problems with the increased prevalence of heroin, cannabis and synthetic drugs (since the 1990s) have further consolidated negative attitudes towards drug use in Vietnam (Hong, Nguyen, \& Ogden, 2004). Consequently, authorities have begun to chart increasing numbers of people who use drugs (PWUD) and the development of new popular drugs. Following regional trends, opium smoking has been replaced by injecting heroin, and this has arguably triggered increasing HIV infection rates among people who inject drugs (PWID) and the

\footnotetext{
${ }^{1}$ During this war, the Democratic Republic of Vietnam controlled the North of Vietnam, while the Republic Vietnamese Government backed by the USA took control of the South. This division led to differences in the drug situation and drug policy between two parts of the country. In the north, the government continued to deploy selective drug policies between lowland and highland regions. In the south, although the government prohibited drugs on paper, drug consumption and production were generally tolerated.
} 
Vietnamese population at large (Windle, 2015). These conditions have further entrenched drug use as a serious social problem.

Image 1.1 Drug flows to Vietnam and the rest of the Southeast Asia region from the Golden Triangle area (Luong, 2017). ${ }^{2}$

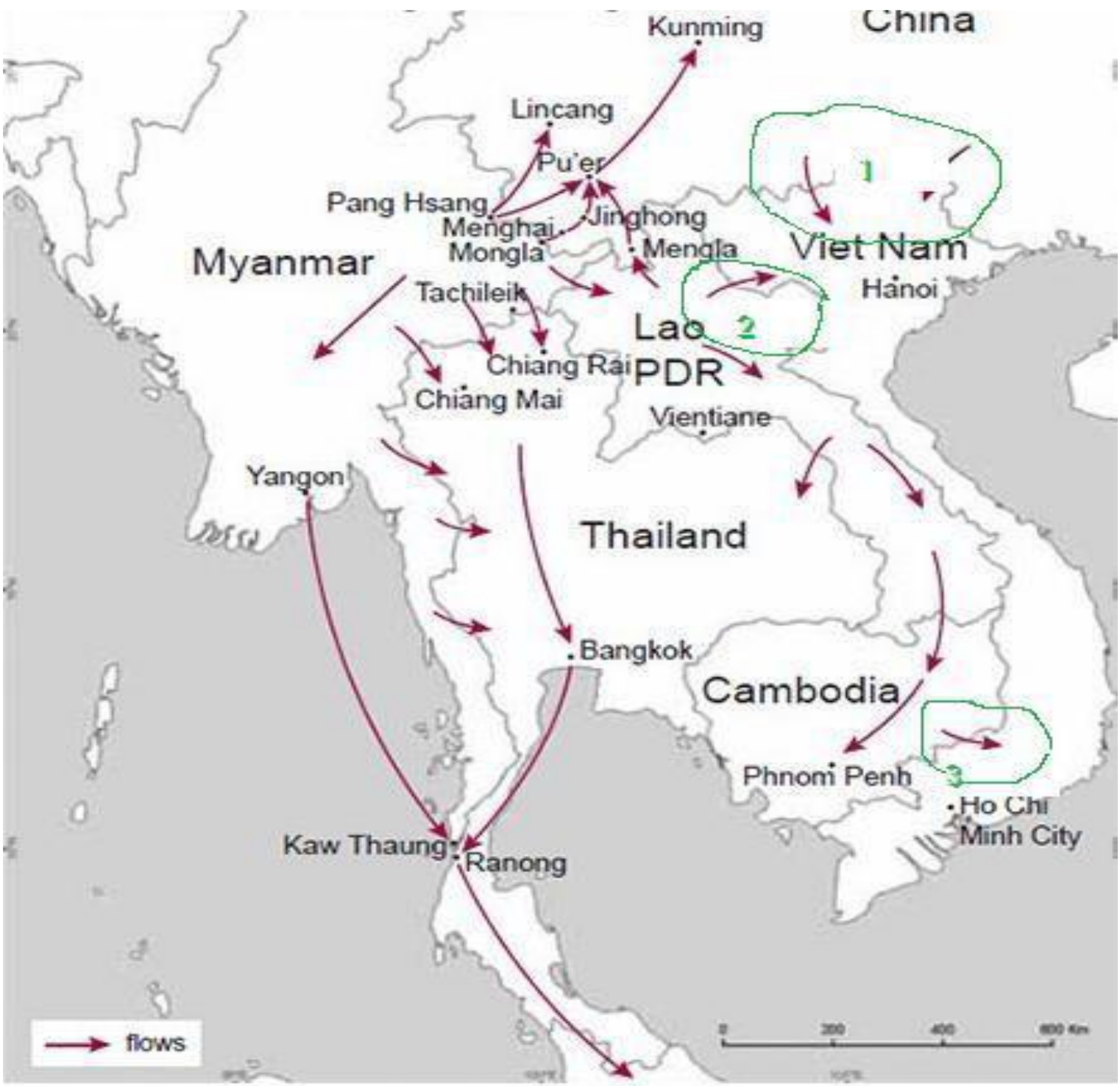

\footnotetext{
${ }^{2}$ The map illustrates the scope and direction of drug movements from other countries to Vietnam. The 'Golden Triangle' (consisting of Laos, Myanmar and Thailand) is a significant site of opiate production, particularly of heroin. This affects illicit drug production and consumption in Vietnam. Illicit drugs produced in the Golden Triangle are trafficked into Vietnam through Laos and Cambodia. Also, illicit drugs transit through Vietnam before being smuggled into other countries like China and other Asian countries. This creates the complex challenge of drug trafficking, consumption and production across Vietnam and the region.
} 


\section{Vietnam's Responses to Drug Use}

Like other Asian countries, Vietnam's drug policy has historically focused on supply and demand reduction measures (abstinence-based approaches), which aim to prevent drug use and seek a society free from drugs. Since the early 1990s, drug use has been cast as a 'social evil', ${ }^{3}$ and people who use drugs (PWUD) have encountered intense police crackdowns and internment in compulsory rehabilitation centres (known as 06 centres). Specifically, in 1992, Article 61 of the 1992 Vietnamese Constitution declared drug use a 'dangerous social disease' and a social evil that needed to be eradicated immediately (Windle, 2015). By and large, this legal foundation considered drug use from a moral perspective. PWUD were viewed as having lost control over their drug use behaviour, so the only hope to overcome it and regain control was to stay completely away from illegal drugs. As a result, the Vietnamese government conducted an intensive campaign against drug use in which law enforcement and punitive approaches were deemed the most effective methods.

Since the 2000s, under the influence of American knowledge related to drug use and addiction, Vietnam has changed its approach on paper, from viewing addiction as a criminal issue to viewing it as a public health one (Pham et al., 2010). That is, drug addiction is now regarded as a brain disease, that results from biological or psychological defects. Accordingly, solutions or interventions against addiction in Vietnam are generally placed at the level of the individual by repairing their biological or psychological defects, rather than addressing the difficult social and environmental circumstances in which they live (Windle, 2012). It is important to note that, although Vietnam has changed how it views addiction on paper, the moral and brain disease models of addiction exist simultaneously and establish a strong foundation for the prevalence of abstinence-oriented responses or prohibition on drug use in the country (USAID, 2011). Therefore, similar to global responses, Vietnam has continued to implement a wide range of prohibitionist measures to prevent drug use (Vuong, Ali, Baldwin, \& Mills, 2012; Windle, 2015).

\footnotetext{
${ }^{3}$ In Vietnam, 'social evil' is a popular term that relates to social phenomena that are opposed to the moral tradition, social conventions and the culture of the state. 'Social evils' are seen to induce adverse consequences in society, and the term is applied to activities such as drug addiction, gambling, prostitution, or alcohol abuse.
} 
Supply reduction is considered an essential component of drug control policy in Vietnam (Duc, 2012). It is commonly believed that the root causes of drug use problems can be addressed through stricter regulations and law enforcement (Khuat et al., 2012). In fact, Vietnam is one of only a handful of states that have successfully suppressed illicit opium production, achieving a decline of $98 \%$ in production (shown in Chapter Five) from the 1980 s to the 2000 s (Windle, 2012). However, other than that, Vietnam has failed to reduce the supply of illicit drugs. According to the United Nations Office on Drugs and Crime (UNODC, 2017), drug crimes like drug trafficking and smuggling have significantly risen since the 1990s in Vietnam. The reasons for this increase include the increases in policing or law enforcement activities ${ }^{4}$, and a higher demand for drug use in Vietnam (Windle, 2012). Alongside supply reduction measures, the Vietnamese government has launched large-scale propaganda campaigns to prevent 'social evils', and agencies continue to develop a system of forced compulsory treatment (Edington, 2016).

Nevertheless, as Windle (2015) argues, these enforced abstinence-based approaches (prohibition, criminalisation, punishment, incarceration or rehabilitation) have had limited effectiveness, and they cause social harm to PWUD. PWUDs are isolated from their families and communities and detained in compulsory treatment centres for treatment purposes. The Vietnamese government tends to believe that sending users to drug treatment centres or 06 centres will reduce the harm caused by drug use, so the 06 centres have been expanded nationwide, increasing from 56 centres in 2000, to 109 centres in 2009, to 123 centres in 2017 (Edington \& Bayer, 2013; Ha et al., 2010; Ministry of Health, 2018b; Reid \& Higgs, 2011). At these centres PWUD are detained as a form of 'administrative sanction'5 and they can be detained in the name of treatment for up to two years for the first relapse and between two and five years for 'a second relapse' ${ }^{6}$ The government also argues that sending PWUD to 06

\footnotetext{
${ }^{4}$ This means that higher levels of law enforcement have ensured detection of higher numbers of drug crimes by police and other relevant agencies

${ }^{5}$ The 2012 Administrative Violation Law categorises drug use as an administrative violation, and people who use drugs can be subjected to a warning, fines or administrative detention for up to two years.

${ }^{6}$ Second time apprehension for using a banned drug.
} 
centres is not only better for PWUD (Reid \& Higgs, 2011), but also better for users' families and communities (Khuat et al., 2012).

However, these centres are not effective in preventing relapse. Despite their intensive and lengthy stays at 06 centres, $90 \%$ of clients fail to abstain from drugs following their release (Windle, 2015). The centres are also heavily criticised for being costly, increasing stigma and discrimination, and violating human rights (Edington, 2016; International Network of People Who Use Drugs, 2014; UNAIDS, 2016; WHO, 2009). On release, PWUD often relapse, are unable to find employment and struggle to reintegrate into society. While abstinence may be an option for some PWUD, the presumed effectiveness of forced abstinence has been demonstrated to be driven more by flawed assumptions and political populism, rather than evidence and rationality (Coomber, McElrath, Measham, \& Moore, 2013; Hutton, 2017; Taylor, Buchanan, \& Ayres, 2016). Forced abstinence produces and exacerbates drug-related harms and remains among the most significant contributor to the harms associated with illicit drug use, like the isolation, stigma or discrimination against those taking drugs (Levy, 2014).

A recent study conducted by Reid and Higgs (2011) highlighted the misunderstandings of Vietnamese stakeholders. The researchers explored how Vietnam adopts harm reduction with the participation of 22 key stakeholders from the government, public health, public security sectors, and international sectors. Their results showed that many participants thought that sending PWUD to detoxification and compulsory drug rehabilitation centres, (06 centres), was a form of harm reduction. For instance, a government worker explained:

... 06 centre is a kind of harm reduction intervention. To PWUD, it brings benefits such as access to detoxification services, health care, nutrition, rehabilitation, behavioural change and education. It can be said that lives are saved and changed in the 06 centre (Member of Provincial AIDS Committee of Ho Chi Minh City, cited in Reid and Higgs (2011, p. 170).

Further, in Edington and Bayer's (2013) Vietnam based study, respondents stated that PWUD would be detoxified and morally re-educated in 06 centres, and that this was a way to resist the 'social evils' and minimise the harm of drugs. Many police officers claim that harm 
reduction interventions like providing needles and syringes to PWUD is 'showing the path to deer to run away', in other words, such provisions foster drug use.

These fundamental misperceptions in the interpretation of 'harm reduction' have led to a series of misleading interventions in practice. Harm reduction is misunderstood and seen as a mechanism through which the government controls drug use and PWUD to protect the wider community (Windle, 2015). Such a focus on law enforcement and forced detoxification is contrary to Vietnam's commitment to 'decriminalise ${ }^{77}$ drug use, violates PWUD' rights and exacerbates their problems (having been recorded as compulsory detainees, PWUD inevitably struggle with community integration). Therefore, although a harm reduction rhetoric has been adopted, it is reluctantly embraced to reduce medical problems and protect the wider society. ${ }^{8}$

\section{On the Rise of Harm Reduction ${ }^{9}$}

'Authentic' harm reduction, an approach that emerged from the 1980s when the HIV/AIDS epidemic became a serious problem, is seen by some governments, such as the United Kingdom, Switzerland or the Netherlands, as an effective approach to address the complexity

\footnotetext{
7 According to the 1999 Vietnamese Criminal Law, drug possession for personal use is decriminalised and drug use is seen as a public health matter, not a criminal one. This will be further discussed in Chapter Five.

${ }^{8}$ Harm reduction is defined in the 2006 HIV Law (Article 2, clause 15) as 'a method that encourages the use of condoms, sterile needles and syringes as well as treatment for people who use drugs by using substitute substances and other interventions with the aim of minimising risk behaviours that can cause HIV infection'. It is evident that the definition of harm reduction is based on a narrow public health perspective concerned with protecting society from HIV infection. It does not refer to reducing other harms associated with illicit drug use, such as harm to society, to the economy or to people who use drugs.

${ }^{9}$ In line with the deployment of demand and supply reduction measures, Vietnam has implemented some medical interventions in the name of harm reduction as a response to the HIV epidemic since the 2000s. The shift from drug smoking to injecting has been regarded as the principal driver of the HIV epidemic in Vietnam. The first case of HIV infection was reported in Ho Chi Minh City in 1990 (Maher, Coupland, \& Musson, 2007; Nguyen \& Wolffers, 1994). During the 1990s, Vietnam experienced a rapid spread of HIV infection among both people who use drugs and the general Vietnamese population. By 1999, HIV infection had been recorded in all provinces and cities (VAAC, 2011). However, since 2006, the rate of new HIV infections has declined because Vietnam has implemented some harm-reduction interventions like needle exchange and condom distribution programmes (Ministry of Health, 2018b; VAAC, 2011).
} 
of drug use behaviours and meet the needs of PWUD (Ritter \& Cameron, 2006). Authentic harm reduction seeks to mitigate the harms caused by drug use within a framework that promotes human rights and humanistic values towards PWUD rather than prevent drug use (Hunt et al., 2003; Wodak, 1998). Authentic harm reduction is based on three main principles, including (i) viewing drug use as a reality of human nature; (ii) treating PWUD with nonjudgemental attitudes and no punishments for drug use; (iii) placing PWUD at the centre of addressing their problems. Importantly, it also aims to reduce the harms caused by prohibitionist drug policies.

However, authentic harm reduction is often misguidedly seen by governments and leaders as any programme and policy that is intended to reduce harm associated with drug use and problem behaviour (Riley et al., 1999). This perception leads to 'pseudo' harm reduction in practice, meaning that they (governments/politicians) pursue and consider punitive prohibition (punishment, incarceration or coercive treatment) to reduce harm caused by drug use (Ritter, Lancaster, Grech, \& Reuter, 2011). Such misperceptions of authentic harm reduction are common but dangerous, because they lead to human rights violations and social injustices, such as the use of indefinite detention for those who use illicit drugs (Buchanan, 2016a). Unfortunately, this misconception is common in Vietnam, where so-called harm reduction is implemented within a narrow public health perspective, and misunderstandings about key elements of authentic harm reduction are widespread.

In Vietnam, so-called harm reduction interventions are mainly implemented by the Ministry of Health $(\mathrm{MOH})$, with the support of the Ministry of Labour, Invalids and Social Affairs (MOLISA) and the Ministry of Public Security (MPS). In practice, some studies conducted by Reid and Higgs (2011) and Edington and Bayer (2013) show that MOLISA and MPS do not properly understand authentic harm reduction, and they tend to use harm reduction as a means to control PWUD. In short, they conduct pseudo harm reduction. In addition, as noted above, the Vietnamese form of harm reduction emerged as a response to the HIV epidemic, and external funding for harm reduction was used for HIV prevention, and more recently for addiction treatment. Further, although Vietnam has conducted some interventions in the name of harm reduction, they used harm reduction as a mechanism to manage and control PWUD, rather than providing them with the necessary assistance (further discussed in 
Chapter Five). In general, misunderstanding about harm reduction philosophies and its principles is a significant issue in Vietnam.

The fundamental principles of harm reduction seem to be rarely articulated in policy and practice in Vietnam, and misconceptions are common. As Reid and Higgs (2011, p. 5) highlight, 'the conceptual understanding of harm reduction for many people from policymakers to those involved in the implementation of the programs is poor'. Moreover, as Edington and Bayer $(2013$, p. 78$)$ concluded from their research, 'local misunderstandings were evident in the confusion over harm reduction's most basic principles as well as the failure to grasp its more far-reaching dimensions'. This is the first piece of work that takes a mixed approach to conduct interview and survey professionals from a wide range of Vietnamese and international agencies related to the drug field. Although there were some small studies that look up some aspects of drug use, user behaviours or drug policy in Vietnam, this is the first study that approaches different agencies and explores their views on much wider perspectives.

This study allows us to find out how professionals perceive drug use, those taking drugs, corresponding responses, and to see what their philosophy is. Importantly, the study points out the differences between what is presented on paper and what professionals do and think practically in relation to harm reduction strategies in Vietnam. This study demonstrates that although Vietnam has adopted harm reduction in theory, it has not yet applied many important elements of authentic harm reduction in practice. This has led to pseudo harm reduction measures, which use punishment, incarceration or coercive treatment as forms to reduce harm. These forms of pseudo harm reduction actually increase and exacerbate the harms from illicit drug use rather than reducing them.

\section{Objectives of the Study}

The purpose of this thesis is to provide a systematic and critical review of harm reduction philosophies and strategies in Vietnam. That is, this thesis compares the critical components of authentic harm reduction (how to conceptualise drug use, how to respond to drug use, and how to treat PWUD) with how 'harm reduction' is perceived and practised in Vietnam. This comparison contributes to identifying the differences, conflicts, gaps, and commonalities in 
the understanding of key stakeholders who work in drug use related fields in Vietnam about harm reduction.

In reality, Vietnam does not really consider that people can take drugs experimentally or recreationally, so harm reduction is applied to all PWUD, who are widely labelled as 'addicts' among Vietnamese people. While acknowledging the major prevalence of recreational drug use (that most users engage with drugs in controlled and recreational ways), this study focuses on problematic PWUD because they especially need help and harm reduction services.

In order to promote authentic harm reduction in Vietnam, this study uses mixed qualitative and quantitative approaches to find out drug use related perceptions among professionals, practical challenges, conflicts, and barriers of current laws, policies, practices, and understanding about implementing harm reduction strategies. Through the findings, this study proposes recommendations as to what Vietnam should do to implement authentic harm reduction strategies in the near future.

\section{Research Questions}

This thesis concentrates on how professionals perceive drug use, drug users, official responses, and harm reduction in conjunction with harm reduction philosophies in Vietnam. Vietnam has embraced harm reduction since the 1990s, but the effectiveness of harm reduction policies has so far been limited. In order to explore what is happening in Vietnam in terms of understandings about and implementation of harm reduction among professionals, a key research question is:

How do key stakeholders perceive and implement harm reduction in Vietnam?

Answering this key research question will uncover whether or not professionals in the medical, social policy and law enforcement fields are implementing harm reduction in Vietnam from adequate understandings, particularly with regard to conceptualising drug use, responding to it, and treating PWUD.

In order to properly explore harm reduction in Vietnam, two further research questions are addressed: 
- What are the limitations of current perceptions on harm reduction, and how does this impact on the implementation of harm reduction strategies?

- What philosophical or practical changes might facilitate improvements in the delivery of harm reduction strategies in Vietnam?

As noted previously, the fundamental principles of harm reduction seem to be rarely articulated in policy and practice in Vietnam, and misconceptions are common. Further, although Vietnam has implemented harm reduction programmes for nearly three decades, but the programmes have produced limited outcomes and encountered many barriers in practice. Therefore, addressing these secondary research questions will contribute to identifying significant challenges and barriers to effective harm reduction, and importantly aid in proposing practical solutions for enhancing authentic harm reduction in Vietnam.

\section{Thesis Overview}

My decision to specifically focus on harm reduction in this study was informed by the following major questions: Why has my country, Vietnam, always struggled to address drug use problems? What are the current problems in Vietnamese drug policy, and how can we explain these problems? As a police officer, I am tasked with preventing illicit drugs and drug use at any cost. Nevertheless, during my PhD journey, three major issues became evident. Firstly, abstinence, which Vietnam follows, does not work in practice. It is mostly based on myths and driven more by political, cultural and moral factors rather than factual pharmacological or scientific evidence. Under an abstinence model, illicit drug use is punished, and users have to make a life-long commitment to be drug-free to combat their addiction. These ineffective responses have had no positive impact on the actual rates or nature of drug use and have created significant harms towards PWUD and society.

Secondly, harm reduction has been regarded as a pragmatic and effective measure for resolving fundamental drug use problems in many countries, like Canada, Netherlands, Switzerland or England. Its principles are based on the fact that drug use is very commonplace in society and is recorded throughout human history (Gossop, 2012). Therefore, it is unrealistic to stop people from using drugs; instead, finding ways to live with all drugs and reducing their harms is more practical (Hofschulte, 2012; McNeece, 2003; Stevens, Stöver, \& 
Brentari, 2010). From harm reductionists' perspectives, addiction and other harms caused by drug use are addressed if the government respects human rights and considers biological, social and psychological factors (the settings) as it treats addiction and PWUD. Harm reduction interventions have generally succeeded in reducing HIV or Hepatitis C infection and overdose (Cavalieri \& Riley, 2012; Crofts \& Azim, 2015). They are also effective in building relationships and understanding between service providers and PWUD (Allman et al., 2007). Harm reduction has gradually integrated into drug policy reform in many countries as a primary response to drug use (International Harm Reduction Association, 2016). As this thesis demonstrates, harm reduction is a better approach to PWUD and the wider society.

Thirdly, like other countries, Vietnam has adopted some interventions in the name of harm reduction due to the HIV epidemic during the 1990s. Yet it also has become clear to me that Vietnam has not yet embraced an authentic harm reduction philosophy. As the study findings show, some so-called harm reduction interventions are firmly rooted in a narrow public health perspective, and confusion about harm reduction principles are widespread. Generally, Vietnamese people have implemented so-called harm reduction under an abstinence paradigm. They believe that harms can be limited through the reduced demand and supply of illicit drugs. These misperceptions have led to intensive incarceration, punishment, and rehabilitation towards PWUD, all seemingly under the guise of harm reduction. Wherever harm reduction is in operation, the Vietnamese government uses it as a mechanism to supervise and control PWUD, in order to force them to join mandatory drug treatment rather than helping them to overcome their economic, social and psychological problems. The Vietnamese version of harm reduction serves political motives and emphasises the protection of the wider community over those who take drugs. In short, I would argue that Vietnam has conducted what could be called pseudo harm reduction.

This thesis is concerned, therefore, with the Vietnamese context. Taking this focus, it seeks to reflect on how illicit drugs, drug use, and PWUD are perceived through historical, social and cultural perspectives. These contextual approaches contribute to an understanding of why abstinence-based approaches are dominant in Vietnam. Some interventions in the name of harm reduction have been adopted in responding to the HIV epidemic during the 1990s, but the prevalence of abstinence-based mind-sets, which consider drug use and users from moral and disease perspectives, has driven Vietnam to implement pseudo harm reduction. This 
study confirms this fact, yet it also shows some opportunities for Vietnam to develop harm reduction in ways that are parallel to an authentic harm reduction philosophy.

\section{The Chapters}

To develop the above ideas, the following Chapter Two discusses the historical and social context of drugs, the notion of drugs, and drug use from global perspectives. By providing the historical and social contexts of drug use, the thesis illustrates that drug use is an indispensable part of people's lives. Yet drug use is widely viewed as a moral and disease problem, instead of a social and psychological one, leading to the prevalence of moral and brain disease models of addiction that overlook the importance of socio-psychological responses. These models, which consider addiction from moral and disease perspectives, have influenced how drug use and addiction have been treated worldwide, which focuses

solely on abstinence. Abstinence-based approaches require PWUD to stay away from drug use completely, so drug use behaviours are punished. Yet this chapter argues that illicit drugs and drug use are socially and politically constructed and these constructions lack any pharmacological clarity, science, and evidence. The chapter also argues that stringent abstinence-based responses have been ineffective and have failed to demonstrate any significant reduction in the harms of drug use. These responses have arguably caused societal harms far greater than the harm caused by illicit drug use.

Chapter Three illustrates the historical and social conditions in which authentic harm reduction is used as a new response to drug use. It details the reasons why some ways of implementing harm reduction in practice remain focused through a narrow public health perspective, and why the confusion and misperceptions about harm reduction are still widespread, including in Vietnam. In particular, this chapter argues that harm reduction is more effective than abstinence in responding to drug use and addressing the long-term problems related to PWUD. It also presents guidelines on how harm reduction should be philosophically defined and understood and practically implemented. These philosophical and practical guidelines are essential because many countries, including Vietnam, have mistakenly interpreted the authentic harm reduction philosophy, leading to pseudo harm reduction in practice. 
Chapter Four illustrates the broader historical and sociocultural contexts of drug use in Vietnam. These illustrations are important to understand why Vietnamese people have negative attitudes towards drug use, leading to punitive responses to users. The chapter further examines how drugs and drug use are socially, culturally and politically constructed in Vietnam. Drug use is viewed as an external contamination, and widely perceived by government and media as the cause of addiction and crime. Given these constructions of drugs and drug use, this chapter shows that Vietnam has developed deeply punitive and abstinence-focused drug policies, leading to pseudo harm reduction strategies.

The social, political and legal constructions of drugs and drug use are highlighted in Chapter Five to further illustrate the prevalence of abstinence-based responses in Vietnam. Vietnamese drug laws and policies mainly focus on two main domains: supply and demand reduction. Yet the chapter argues that Vietnamese abstinence-based approaches have had limited effectiveness in reducing supply and demand, and the harms associated with illicit drugs. These approaches have caused negative consequences for PWUD, especially stigma, discrimination, and human rights violations. Although Vietnam has implemented some interventions in the name of harm reduction, the current understanding of harm reduction is firmly rooted in a narrow medical perspective, and misperceptions and misapplications are widespread. This chapter argues that the current understanding and official implementation of harm reduction in Vietnam is not authentic harm reduction at all.

Chapter Six outlines the critical methodological approaches used for this research on harm reduction in Vietnam. It highlights the importance of the positivist and interpretivist methodological approaches for this study and establishes the nature of my practical methods. It also illustrates the difficulties of doing research in Vietnam and accessing study participants (police, local authorities, social workers, public health workers, and international officials). These difficulties partly contribute to explaining why harm reduction faces strong opposition from police and workers working for the MOLISA in practice. The chapter also illustrates my personal reflections on conducting the research and how I have changed my mind-set and approaches to drug use during my PhD journey.

The findings of this study are presented from Chapter Seven with details about how survey respondents and interviewees perceived drug use, addiction and PWUD. This chapter shows that, while drug use is regarded as a public health matter on paper, the majority of survey 
and interview participants view it as a social evil and they hold many myths about drug use and addiction. Their mythical perceptions encompass: (i) illicit drugs are the most harmful, (ii) cannabis causes death, (iii) recreational use of illicit drugs is impossible, (iv) illicit drug use inevitably leads to addiction, and (v) illicit drug use causes crime. These findings reaffirm the fact that abstinence-based mind-sets and judgemental attitudes remain popular among Vietnamese professionals. As a result, Vietnamese responses to drug use are generally punitive, to which the next chapter turns.

Chapter Eight illustrates what study participants thought Vietnam should do to respond to drug use. Working from problematic understandings about drug use, many professionals tended to promote punitive control measures to reduce the harms associated with drug use. They believed that reducing harm means eradicating the supply, and controlling and punishing PWUD by sending them to forced treatment. Yet the chapter argues, while the Vietnamese government has made great efforts to prevent drug use and promote coercive treatment, it has failed to curb the drug use situation in the country. Particularly, its punitive approaches have caused many social harms to PWUD, such as unemployment, blood-borne disease transmission, social dislocation or stigma, and discrimination. Fortunately, some interviewees had progressive thinking, as they considered psychological and social factors in treatment and promoted PWUD' rights in deciding their treatment. These perceptions are significant, as they can pave the way for promoting 'authentic' harm reduction strategies in Vietnam.

Chapter Nine evaluates how the study participants perceived a harm reduction philosophy and how they applied it in practice. By and large, study participants often misunderstood the harm reduction philosophy. Harm reduction, for them, refers to any measure that can reduce drug use and drug crimes. It is also clear that, while most international and public health workers supported harm reduction as a means to reduce HIV transmission and to treat addiction, police and MOLISA workers remained sceptical of the benefits of harm reduction. These sceptical perceptions become clear when police and MOLISA workers were asked about drug consumption rooms, clean injectable drugs, and naloxone. In addition, the chapter illustrates other practical barriers that hamper harm reduction from moving forward. This chapter ends with recommendations made by interviewees on how Vietnam should reform its harm reduction approaches and drug policies. 
Chapter Ten concludes with a discussion of how harm reduction was perceived and implemented in Vietnam. By using the current practice of harm reduction in Vietnam and findings from this thesis, the chapter argues that Vietnam has embraced the wording of harm reduction, but its understanding and implementation of harm reduction are driven by abstinence-based mind-sets. This has resulted in pseudo harm reduction that is used as a mechanism to prevent drug use and to control PWUD. The Vietnamese model of harm reduction is generally not parallel with authentic harm reduction strategies. In response, the chapter reemphasises some key components of authentic harm reduction and why they are so vital. It also raises some successful international examples from other countries, where authentic harm reduction is implemented properly. These examples provide good models for Vietnam to follow and reform its drug policy, as well as confidence in the effectiveness of authentic harm reduction approaches. Finally, the chapter suggests recommendations that might contribute to the receptivity of the Vietnamese policy-makers to the findings of this study and promote authentic harm reduction. Importantly, the chapter highlights how Vietnam can move forward to be a country that practises authentic harm reduction. 


\section{Chapter Two: Abstinence: A Global Response to Drug Use}

\section{Introduction}

Since the beginning of the $20^{\text {th }}$ century, global responses to drug use have tended to engage with abstinence-based perspectives (Levy, 2014). Abstinence is an approach that aims to prevent drug use and seeks a society free from drugs (Inciardi \& Harrison, 1999). According to abstentionists, it is seen as the most effective method to resolve drug use problems, such as health damage or offending (Dolan, Larney, \& Wodak, 2007; Wodak, 2011). Globally, enforced abstinence-based approaches (like criminalisation and punitive responses to drug use) continue to be implemented, despite the fact that there is little scientific evidence to demonstrate that they have yielded positive outcomes (Global Commission on Drug Policy, 2014). It is important to note that the popularity and prevalence of abstinence seems to largely rest on how addiction is popularly perceived and theorised.

Abstinence-oriented approaches are derived from models of addiction that emphasise moral codes and also brain disease. The former regards drug use as the violation of social rules and norms that lead users to lose control, so for many governments punishment and incarceration are seen as the best methods to help PWUD to regain control over their behaviours (Bauer, Eppler, \& Wolf, 2007). The moral model arguably has limited value for addiction treatment because it overemphasises punitive methods and mainly serves political rationales rather than adhering to the evidence. The latter model of addiction highlights that brain and genetic abnormalities lead people to become addicted to drugs, and therefore for abstentionists, abstaining from all drug consumption or ensuring a life-long commitment to be drug-free is seen as among the most effective addiction treatment methods (West \& Brown, 2013). The brain disease model mainly focuses on individualising and medicalising addiction while the social and environmental factors that shape addictive behaviours seem to be overlooked in treatment, particularly in Asian countries, Vietnam included (Clark, 2011).

Understanding those dominant models is important because it paves the way for recognising why abstinence and punitive based approaches are widespread in most countries, including Vietnam. In fact, abstinence can be an option for some drug users, but forced abstinence has also been demonstrated as being ineffective and having failed to demonstrate any significant 
reduction in drug use, addiction, drug supply or the harms of drugs (Buchanan, 2016b; Count the Costs, 2010; Fedotov, 2010; Wodak, 2011, 2014). Indeed, these forced abstinence responses have arguably caused societal harms far greater than the harm caused by illicit drugs in the first place (Rolles, 2012).

This chapter will first review the historical and social context of drugs and the notion of drugs, which help to understand why some drugs are categorised as legal while others are illegal and seen as negative things. Next, the chapter explores the drug use phenomenon, and its role in shaping people's perceptions about drug use, addiction and PWUD. In fact, the way people acknowledge and perceive drug use will widely affect the way they define addiction and how they respond to those using drugs. Therefore, the chapter will also examine the two current popular models of addiction (particularly in Asian, Vietnam included), namely the moral and the brain disease models of addiction, which have a strong influence on how governments respond to drug use (Clark, 2011). These models have also, via US-involvement, influenced Vietnam's drug policy both in philosophy and practice (further discussed in Chapter Four). This is because, within a Vietnamese context, drug use is regarded as a 'social evil' while addiction is documented as a brain disease model (Nguyen \& Scannapieco, 2008; Vuong et al., 2012; Vuong et al., 2016). The chapter also examines a psychological and sociological model of addiction that considers addiction from wider perspectives, but this model seems to be ignored across many countries, including in Vietnam. The rhetoric of drug use and addiction in Vietnam will be critically discussed in later thesis chapters. However, understanding the history and the critical principles of drug use and each addiction model will provide a basis for exploring why abstinence remains the dominant paradigm in spite of its ineffectiveness and poor record in solving drug use related issues (Levy, 2014).

This chapter argues that the classifications of illicit drugs are based on social and political constructions rather than evidence. In fact, it is important to note that drug use does not inevitably lead to addiction or crime, and only a minority of PWUD can be called 'problematic' (UNODC, 2016b). The misperceptions of drugs and drug use result from the prevalence of the moral and brain disease models of addiction, and they result in a raft of unintended consequences. 


\section{The Notion of Drugs}

Many drugs (currently seen as illicit drugs) play an important part in society and in the lives of many people (Gossop, 2012). In fact, the use of drugs for recreational, medical and social purposes is recorded throughout human history. Archaeological evidence showed that Stone Age peoples consumed hallucinogenic mushrooms more than 12,000 years ago (McKenna, 1999), while opium has been used for 4,500 years, for pleasure and medical purposes (Rudgley, 1994). In the Bronze Age, the opium poppy was consumed widely as a balm for the pains of childbirth and of disease (Lindesmith, 1965). Cannabis or hemp was grown in China and also in Neolithic Europe almost 4,500 years ago for local rituals (Rudgley, 1994). Human ancestors consumed drugs not only to seek altered mental states and for medication, but also for survival and for adapting to harsh environmental conditions. Drugs even became important sources of nutrition and energy (Thomas, 2002). Historically, opium from the immature fruits of the opium poppy, cannabis from the Indian hemp plant or cocaine from the leaves of the South American coca plant were extracted and used as local vegetables. This historical evidence demonstrates that drugs have been used and enjoyed throughout the ages.

Internationally, the 'United Nations Single Conventions on Drugs' - like the 'Single Convention on Narcotic Drugs 1961', the 'Convention on Psychotropic Substances 1971' and the 'Convention against the Illicit Traffic in Narcotic Drugs and Psychotropic Substances 1988' have changed the landscape of drug use (Taylor et al., 2016). These Conventions list narcotic and psychotropic substances that require strict legal controls for medical or research purposes due to their perceived harmful effects on human health (Roberts, Klein, \& Trace, 2004). In order to justify these Conventions, the United Nations (UN) formally stated that illicit drugs are a grave threat to the health and wellbeing of all people, they destroy lives and communities, undermine sustainable human development and generate crime (Gurule, 1998). Such emotional language has emerged through individual Conventions. For example, the 1961 Convention asserts that 'narcotic drugs have no place in society and must be restricted to medical and scientific purposes' (Taylor et al., 2016, p. 3). This Convention also refers to drugs as: 
A serious evil for the individual, and a threat which the international community has a duty to combat because it is fraught with social and economic danger to mankind (United Nations, 1961, p. 1).

The use of such language has created the mark of social disgrace by presenting PWUD as a threat to society (Count the Costs, 2010). The Conventions foster (and to some extent require) criminal sanctions to be put in place at the national level. For instance, Article 3 of the 'Convention against the Illicit Traffic in Narcotic Drugs and Psychotropic Substances 1988' states that:

Each Party shall adopt such measures as may be necessary [such as treatment, education, after-care, rehabilitation and social reintegration] to establish as criminal offenses under its domestic law [pertaining to possession and purchase of drugs] subject to [the nation's] constitutional principles and the basic concepts of its legal system (United Nations, 1988, p. 3).

It is evident that these Conventions have contributed to a significant shift away from drug accommodation and regulation towards a more punitive approach (Bewley-Taylor \& Jelsma, 2012), paving the way for the legislative foundation for contemporary global responses to illicit drugs (Taylor et al., 2016). Based on those assumptions about the negative consequences of illicit drugs as unsafe, extremely harmful, leading to addiction or causing crime, the UN Conventions have promoted a prohibitionist framework and drug-free philosophy (Coomber, 1998). In their global reach, these Conventions have also underpinned the formulation and development of 'local' drug policy of member states, Vietnam included. Although relatively little has changed for almost five decades, recent years have witnessed 'a wave of alternative drug policies, including models of decriminalisation (e.g. Portugal, Spain, Jamaica, Netherlands), legalisation (e.g. Uruguay, US States) and regulation (e.g. New Zealand)' (Taylor et al., 2016, p. 4). Nevertheless, there have been no countries that have wholly decoupled themselves from prohibition of all illegal drugs (Buchanan, 2015b). Instead, they (UN Convention members) continue to categorise some drugs as legal while prohibiting other drugs. 
Legal drugs in the form of cigarettes, beer, tea, coffee or chocolate are socially acceptable during times of stress or celebration, but the same cannot be said for illegal drugs (Boland, 2008). As Gossop (2012) argues, legal drugs (particularly so for alcohol and tobacco, which are among the most unhealthy drugs) are generally accepted because they are seen to be (relatively) safe and, importantly, they are good businesses as they contribute a significant amount of tax revenue for the state. Meanwhile, illegal drugs are condemned for leading to addiction and health problems; their medical and recreational benefits are overlooked (Boland, 2008). In fact, within much official and social discourse, there is an 'iron law' that using illicit drugs leads to addiction (Frey, 1997). As Taylor et al. (2016, p. 11) explain, the reason for the common perception 'illicit drugs lead to addiction' is that the use of any prohibited drug is portrayed as dangerous and little distinction is made between use (that is mostly recreational) and addiction. It is also common to see that most doctors, scientists and journalists tend to emphasise the health risks posed by illegal drugs and claim that illegal drugs are chemically harmful and highly addictive (Boland, 2008). Yet an experiment conducted by Professor Alexander Bruce called 'Rat Park' ${ }^{10}$ demonstrated that arguing that addiction is a problem caused by an addictive drug or a person is far too simple. He argues

${ }^{10}$ Rat Park was a study about drug addiction conducted by Canadian psychologist Bruce K. Alexander and his colleagues at Simon Fraser University in British Columbia, Canada, in the late 1970s. Alexander and his colleagues implemented many experiments to test the reasons for rats' willingness to consume morphine. The experiments involved two main groups of rats. The first group was isolated in laboratory cages while the second was housed in Rat Park (an area that had walls with scenes of woodlands, natural environments, and, importantly, in this area the rats could play, mate or interact with other rats as in their natural environments). In this experiment, the rats could drink a fluid from one of two drop dispensers. The first dispenser contained a morphine solution while the second one plain tap water. The team recorded how much each rat group drank. The findings of the experiments were noticeable. The caged rats consumed the morphine and then became dependent on morphine, while the rats in Rat Park did not drink the morphine water. His hypothesis was that drugs do not cause addiction; he argues that addiction is attributable to their living conditions (setting), and not to any addictive property of the drug itself, or to personality (set) (Levine, 2009). The Rat Part study is valuable, although it's criticisms should be acknowledged. For instance, MacBride (2017) pointed out that rats are not human and thus behave differently than humans do, or morphine (an opioid) was the only type of drug used in the experiment, so it is hard to conclude what happens with other drugs. Some researchers tried to duplicate the experiments and they received another result, concluding that the results of 'rat park' experiments may also depend in part on the breed of rat used (MacBride, 2017). Yet the role of environmental factors is undeniable, as demonstrated by the experiences of US soldiers in Vietnam, and this is widely accepted by the majority of researchers. 
that the majority of people become addicted to drugs because they use drugs as a way of coping with their dislocation or as an escape, a painkiller, or a kind of substitute for a full life (Buchanan, 2015b).

Similarly, Zinberg (1986) argues that to understand why people become addicted to drugs, the drug, the set and the setting are three important factors. The drug relates to the pharmacological effects of the substance, the set is the user's personality and attitudes, while the setting relates to the social, psychological and environmental contexts in which drug use occurs. His study demonstrated that although psychopharmacological properties of some drugs can make compulsive use more likely, the role of setting is of crucial importance for drug use and addiction. Zinberg uses the 'Vietnam War' to exemplify the importance of social setting. About $20 \%$ of American soldiers who served in the Vietnam War were addicted to heroin (they used heroin to overcome the constant fear of guerrilla attack), but the overwhelming majority (nearly 90\%) desisted from heroin use after they returned home.

However, the environmental, cultural and contextual issues related to illicit drug use should not be viewed as the only determining factors of addiction. The role of the drug is important, and this is argued by the American Psychiatric Association (APA) (Hammer et al., 2013). Some drugs have higher risks of causing addiction than others (e.g. heroin is highly addictive compared to cannabis) (Nutt, King, Saulsbury, \& Blakemore, 2007). Regarding the personality, based on the 'DSM-5'11, the APA asserts that people are not all automatically or equally vulnerable to developing addiction and that some people are less likely to control their drug use, that makes them more likely to become addicted to drugs than others (Hartney, 2019). Further, it is important to note that arguing only illicit drugs as causing addiction seems to too simple as there is a vast literature and research on addiction to legal drugs like alcohol and tobacco (Bancroft, 2009; Heyman, 2009). For example, the DSM-5 notes that addiction results from the use of ten separate classes of substance, namely alcohol, caffeine, cannabis, hallucinogens, inhalants, opioids, sedatives and anxiolytics, stimulants, tobacco, and different

${ }^{11}$ The Diagnostic and Statistical Manual of Mental Disorders, Fifth Edition, often called the DSM-V or DSM 5, is the latest version of the American Psychiatric Association's gold-standard text on the names, symptoms, and diagnostic features of every recognized mental illnessincluding addictions (Cited from https://www.verywellmind.com/dsm-5-criteria-forsubstance-use-disorders-21926). 
substances can form different types of addiction (Saunders, 2017). Addiction is a complex issue that is influenced by many factors (Alexander, 2010). Although the role of the drug and personality are important when it comes to addiction, but they are not only sources that lead to addiction. This is because other social, environmental and psychological also have strong impacts on addiction (these issues will be further discussed later in this thesis).

In fact, as Taylor et al. (2016) argue, attributing illicit drugs (the drug) and users (the set) to addiction has linked to the disease and moral models of addiction. These models tend to overemphasise the importance of illegal drugs and PWUD for developing addiction (Clark, 2011). As a result, treatment is widely focused on reducing drug consumption and reforming PWUD. Crucially, this discourse and assumption generally provide legitimacy for prohibition. Therefore, in order to reduce the harms caused by illicit drug use, the use of these drugs needs to be controlled and eradicated (Coomber, Hunt, \& Milhet, 2016). This fear of drug use and the perceived inevitability of drug use leading to addiction is also used to justify the drug control policy and the promotion of compulsory treatment centres (Wu, 2013), particularly in Vietnam. Yet drug controls and coercive treatment have been demonstrated as being ineffective and having counter-effects (Levy, 2014) (these issues will be further discussed later).

In addition to blaming illicit drugs for causing addiction, the UN and its member states differentiate between legal and illegal drugs because they argue that illegal drugs cause crime, especially violent crime and social disorder (Boland, 2008); yet, their arguments are contested. The argument related to the drugs and crime connection can be seen across different countries. For example, a 1997 British academic study (conducted for the government) showed that a large proportion of arrestees' criminal activity was attributable to problematic illegal drug use (Bennett, 1998). Accordingly, the UK government warned citizens that illegal drugs 'turn law-abiding citizens into thieves', and that illegal drugs 'contribute dramatically to the volume of crime', and thereby posed a threat to social order and community safety (Boland, 2008, p. 173). Similarly, in 2004, the DUMA (Drug Use Monitoring in Australia) programme revealed that $62 \%$ of arrestees tested positive for illegal drugs (Bennett \& Holloway, 2007). At a global level, the United Nations Office on Drugs and Crime (UNODC, 2007) has also stated that there are direct links between illegal drugs and 
crime. The ASEAN Inter-Parliamentary Assembly (2015) has asserted that drug use has negative impacts on the wider community, resulting in crime, prostitution, neighbourhood unease and anti-social behaviour. The arguments and evidence for the connection between illicit drugs and crime are used to buttress prohibition (Boland, 2008). As Stevens et al. (2010) argue, for many policy-makers, the best way to address drug use problems is to reduce the prevalence of illicit drugs or apply prohibition.

The perceived connections between 'drugs' and 'crime' are such that people are regularly sent messages from leaders, politicians, teachers, public health specialists or social scientists that illicit drugs are evil, or that drugs will cause certain offences (Bancroft, 2009). People are also bombarded with daily sensational headlines associating illicit drug use with serious crimes, such as drug trafficking, acquisitive crimes, human trafficking, sex slaves or money laundering (Boland, 2008). These media representations feed an orthodox attitude in which drug use is regarded to be a major threat to peoples' lives, the safety of society, and to social order (Boland, 2008). In turn, common discourses around those who consume drugs often focus on their deviant or criminal nature.

However, the drug-crime nexus is complex, and little support can be found for a single, specific, and direct causal connection (Bean, 2014; Buchanan, 2008, 2015a; Riordan, 2017). For example, Bennett (1998), Hammersley (2008) and Bean (2014) argue that PWUD who are arrested and test positive with drugs are not always problematic PWUD and their offences have not necessarily been 'caused' by drugs. One study conducted by Wilkins et al. (2015), from the New Zealand Arrestee Drug Use Monitoring data, revealed that in 2015, $17 \%$ of the detainees self-reported that they had shoplifted in the previous month, $15 \%$ of the detainees committed a property crime, the figures for drug trading and a violent crime are $21 \%$ and $18 \%$ respectively. The study also showed that $51 \%$ of the detainees were unemployed or on a sickness benefit, $42 \%$ were employed (12\% part-time and $30 \%$ full-time), and $7 \%$ were students. Further, according to this study, drug related offences accounted for around $16 \%$ of the total NZ crime rates during 2010-2014. The results of the study generally demonstrate that taking drugs does not always result in or is directly associated with criminal behaviour. In fact, there are a great number of illicit drug users who do not have a drug problem and do not commit crime. 
In reality, the complicated links between drugs and crime are contested and depend on many factors (Buchanan, 2015b; Stevens, 2010; Walters, 1994). For instance, Goldstein's (1985) tripartite conceptual framework to explain the drug-crime nexus offered not just one explanation of the drug-crime link, but three: the psychopharmacological, economic compulsive, and systemic violence models (cited in Stevens, 2010). The psychopharmacological model proposes that some people may become excitable, irrational and may exhibit violent behaviour after they consume illicit drugs or/and legal drugs, such as opioids, cannabis or/and alcohol. In other words, the effects of drug use (either legal or illegal) lead to certain types of criminal behaviour like violent crimes. However, people may take different types of illegal and legal drugs, so it is difficult to confirm that only illicit drugs are drivers of crime (Horvath \& LeBoutillier, 2014). The economic compulsive model suggests that some PWUD conduct economic related crime to fund their drug habit. They may commit violent crimes like robbery, or nonviolent crimes such as burglary and/or shoplifting. Yet as Buchanan (2015a) argues, if drugs are legalised, users could afford them and they would not commit crime. Meanwhile, the systemic model explains that the world of the illicit drug market is inherently violent. Traffickers, dealers and users do not base their activities on the legal system, so they tend to regulate their affairs by using violence. Notably, recent research conducted by Riordan (2017, p. 58) on the drug-crime connection reveals that:

... it is no longer accepted within the empirical research literature that the drugs-crime relationship is a simplistic direct causal relationship ... that is complex and multifaceted with indirect causal factors that operate in an idiosyncratic nature over time, across individuals, environments and culture.

Importantly, many researchers like Bean (2014), Riordan (2017), or Stevens (2007) demonstrate that most crimes do not involve drugs connection, and that drugs (even if taken) are not the only reason for crimes; yet they are associated with deeper underlying issues, such as inequality, a lack of social capital and social exclusion. In other words, extreme poverty, blocked futures, exclusion and long-term unemployment are often the conditions that lead some people to addiction. In these communities, crime might be the only way of accessing material possessions, paying bills or survival. As Stevens (2010, p. 35) argues, 'being male, unemployed, an early school leaver and homeless is more important in predicting the frequency of various types of offending than use of any specific drug'. In general, inequality, 
poverty, social disorganisation or dislocation are the drivers of crime as opposed to illicit drug use being drivers of crime.

Furthermore, as Bennett and Holloway (2007), Taylor et al. (2016) and Buchanan (2006) argue, to some extent, there is a drug-crime connection established by the 'prohibition' of illicit drugs (Taylor et al., 2016, p. 460). Similarly, Rolles (2012, p. 10) states that:

Crime is not driven by any pharmacological impact of the drug, but fuelled by the process of tough law enforcement upon outlawed substances: using drug-related crime as a justification for the war on drugs is unsustainable given the key role of enforcement in fuelling the illegal trade and related criminality.

In addition, Kerr, Small, and Wood (2005), Stevens, Bewley-Taylor, and Dreyfus (2009) and Werb et al. (2011) argue that tough enforcement measures and prohibition partly contribute to spawning a 'drug underworld' or 'illegal drug market' in which crime, violence and deviant behaviour emerge as responses to enforcement measures. Buchanan (2015a) also states that prohibition can breed gangsters, violence and money laundering. In general, crimes not only have some connections with the illicit drug, but they are associated with prohibition as well. In other words, these are systemic crimes under Goldstein's tripartite conceptual framework. Therefore, arguing that illicit drugs are a driving force of crime is not always the case; the drugs-crime nexus is complicated and needs to be thoroughly considered from different angles as noted above.

In short, narcotics or controlled drugs are referred to as illegal simply because those drugs are listed in the UN conventions, which are based on the social and cultural contexts of the mid- $20^{\text {th }}$ century, rather than updated pharmacological or scientific evidence. In reality, both legal and illegal drugs have existed and used for recreational, medical and social purposes throughout human history. As with legal drugs, illicit drugs have both benefits and drawbacks, but illicit drugs have been treated differently. They are blamed for causing addiction and crime. Yet, as demonstrated above, there are many factors that lead people to addiction and criminal behaviour. The setting (environment) or psychological and social factors are more important in leading someone to use drugs and commit crime. Addiction and crime are socially and psychologically complex issues, and ones that are influenced by many wider 
social, cultural, economic and political factors. Therefore, it can be argued that the current distinctions between illegal and legal drugs are driven by political factors rather than evidence and the facts about drug use.

\section{Drug Use}

Consuming drugs is an almost universal phenomenon in modern life and the reasons for taking substances are generally similar, for both legal and illegal drugs (Gossop, 2012). On a daily basis, people feel the need to start a new day with a shot of coffee, tea or cigarettes (Bancroft, 2009). Similarly, when people go to bars, clubs or restaurants, they often feel the need to start their conversation with some cigarettes, glasses of wine or a few bottles of beer (Buchanan, 2008). They might repeat these habits daily, because it helps them to unwind (Buchanan, 2015b). People have always used drugs, either legal or illegal, in daily life. As Bancroft (2009) argues, drug use has been closely integrated into human life as a social practice and cultural ritual. In reality, alcohol and tobacco are the most commonly used psychoactive drugs and the reasons for using them mirror the use of other drugs, including illegal substances (Goldberg, 1999). Like cannabis, heroin, cocaine or other synthetic drugs, alcohol and tobacco are taken to relax, unwind or celebrate an occasion, but they also have the potential to lead to addiction and problematic use (Buchanan, 2009).

According to Nutt (2012), people take drugs for two main reasons: to experience pleasure and to relieve suffering, or to put it another way: for recreational and medical purposes. For example, cannabis is widely used for recreation; however, other people can use cannabis to help them reduce suffering (Gossop, 2012; Nutt, 2012). Alcohol is consumed for similar reasons. For instance, Goldberg (1999) highlights that people who suffer from long-term serious anxiety may drink alcohol on a daily basis not only for obtaining pleasure but also, importantly, as an attempt to subdue their suffering. The evidence shows that people vary their drug use, taking drugs according to the context, and that drug use generally intertwines with social contexts (Gossop, 2012). For example, Buchanan (2009) indicates that drug taking can be seen as a social phenomenon, a decision influenced by factors such as choice, peer pressure, boredom, hedonism and social networks. This is particularly the case in Vietnam. Several studies have demonstrated that Vietnamese people, especially younger populations, 
take drugs to impress their peers and to be included within their social networks (Duc, 2012; Hoe, 2013; Thien, 2002).

Although people take legal and illegal drugs for similar reasons, illegal drug use has been treated differently. As noted previously, illicit drug use is argued as causing addiction and crime. However, arguing that drug use causes addiction and generates criminal offences is contested (Boland, 2008; Buchanan, 2015b; Taylor et al., 2016). Firstly, it must be acknowledged that drug use does not often lead to addiction and crime. As Taylor et al. (2016) argue, the majority of PWUD are recreational and sensible drug users, not problematic drug users $^{12}$. For instance, a study on cannabis use in New Zealand conducted by Fergusson and Boden (2011) showed that only $10 \%$ of young people developed a pattern of heavy dependent use. Similarly, Shewan and Dalgarno (2005) conducted a study on heroin users in Glasgow (Scotland) and they revealed that about $75 \%$ of heroin users had normal employment, maintained positive social, friend and family relationships, were not dependent on heroin and did not cause any crime problems to society. In 2015, the UNODC also reported that only around one out of ten PWUD was a 'problematic' user, suffering from drug use disorders or drug dependence (ASEAN Inter-Parliamentary Assembly, 2015, p. ix). Further, it is important to note that there is a substantial literature on addiction to legal drugs (e.g. alcohol or tobacco) and on the relationship between legal drugs and crime, particularly violent crime (Bean, 2014). Therefore, arguing that only illicit drugs to cause crime and addiction is biased and socially constructed. In sum, although taking drugs will have some psychopharmacological effects that can lead criminal behaviour (according to tripartite conceptual framework), there is no rule that regular drug use inevitably leads to addiction or problematic drug use (Hart (2013). Instead, as Zinberg (1986) argues, the set (person) and setting (the environment) are more influential as risk factors than the drug in relation to addiction, as discussed above. In other words, factors like socioeconomic disadvantages, personal trauma, social exclusion, stigma or discrimination precede addiction (Stevens, 2010).

\footnotetext{
12 Problematic drug users are defined as people who are dependent socially, psychologically and/or physically upon a substance or substances, to the extent that they experience problems and/or present problems to others (Buchanan \& Young, 2000, p. 414).
} 
In fact, those who consume illegal drugs are treated differently because they take substances that are argued to cause crime, social problems and addiction, and offer limited benefits to users and others (Taylor et al., 2016). Although the perceptions of illicit drugs and drug use are often socially constructed, and lack credibility or evidence, these misperceptions still remain popular in present drug policy. By and large, this is because misperceptions of addiction are prevalent. In fact, not all drug use leads to addiction and not all drug users are addicted to drugs (Davies, 2013). This means that some people do successfully use drugs recreationally without significant problems from their use. The connection between drug use and addiction is complex (Kuzmarov, 2013). For example, based on the US national epidemiological survey for alcohol and other drugs and the DSM-V, Martin, Chung, and Langenbucher (2008) highlight that it is important to consider how the percentage of individuals who 'ever' try a drug meet the diagnostic criteria for addiction; how many regular users meet these criteria; and what proportion of drug using episodes are perpetrated by problem drug users. Furthermore, as Durrant and Thakker (2003) and Nutt $(2007,2010)$ argue, the proportion of dependent users among all users varies by drug type (e.g. very high for nicotine, high for heroin, much lower for cannabis). Addiction is a complex and multifaceted issue, so understanding it comprehensively is vital for having proper responses or treatment. The next section will explore the two common models of addiction (in Asian countries and Vietnam), namely the 'moral model' and the 'brain disease model', which paves the way for understanding why illicit drug use and people who use drugs are treated unfairly and why punitive approaches to them are widespread. The next section also discusses the psycho-social model of addiction, which considers addiction from broader social and psychological perspectives.

\section{Addiction}

Drug addiction is a complex and contested concept that is influenced by many factors. Therefore, acknowledging the contested nature of addiction is essential to thinking about responses to drug use problems. This is because how addiction is perceived will impact on how people who use drugs are responded to (Peele, 2000). Historically, addiction has been viewed as either a moral problem or a disease and, more recently, as a brain disease. In this section, the moral model and brain disease model, which are the most popular models of 
addiction, are critically analysed. Understanding these models of addiction is important, because they provide a basis to comprehend the current and global perceptions of addiction and how the addicted person is framed (Clark, 2011). Specifically, this section presents a brief history of the topic, before exploring the main perspectives of each model and their key approaches to treating addiction. Both models advocate and promote abstinence. Nevertheless, the history and the benefits of each model demonstrate that, while they can serve political rationales for punishment and prohibition, they have limited value for addiction treatment or to address drug-use related problems (Davies, 2013). Therefore, a psychological and social model of addiction is worth considering.

\section{Moral Model}

The moral model of addiction is the oldest model used to explain addiction. This model ${ }^{13}$ was greatly affected by the Harrison Act (1914), which was a response to the increase in drug use in the United States in the early 1900s (MacCoun \& Reuter, 2001). The Harrison Act became the standard and the basis of narcotic regulation in the United States for the next 50 years (Harrison, Backenheimer, \& Inciardi, 1995). Since 1914, drugs have been controlled more strictly by the law when drugs, especially cannabis, opium and cocaine, became widespread in the United States of America (Siegler \& Osmond, 1968). The Harrison Act ultimately served to make illegal the nonmedical use of morphine and cocaine and required all people who imported, manufactured, produced, compounded, sold, dispensed, or otherwise distributed cocaine and opioid drugs to register with the Treasury Department, pay special taxes and keep records of all transactions (Inciardi, 1991). When the Harrison Act was passed, it was seen not just as a simple regulation, but as a moral breakthrough - a shield against the foreign menaces that threatened American society (Ramaswamy, 2013). The law meant that the use of nonmedical narcotics was wrong, a menace both physically and morally to society (BewleyTaylor \& Jelsma, 2012). The Harrison Act was used as a powerful tool for federal authorities to regulate, and ultimately prohibit drug use (KCBA, 2005).

\footnotetext{
13 The moral model has its origins in theories of alcohol dependence from the 18th century and were influential in temperance movements prominent in the 19th century in the United States and (especially northern) Europe (Harding, 1986).
} 
Before the Harrison Act came into effect, physicians viewed addicted persons as patients, but from 1914 they started to see people who use drugs as immoral persons (Lindesmith, 1965). This Act established the moral model of addiction and prohibition became a dominant theme in the US (Bewley-Taylor \& Jelsma, 2012). As a result of this legislation, addiction became a matter for the police rather than the medical profession. According to Siegler and Osmond (1968), the moral model is also characterised by a religious perspective. That is, addictive behaviours are seen to stem from a drug user's lack of spiritual understanding, which ultimately leads to their 'sin' of drug use. This perspective regards people who engage in drug use as having low moral standards or as being irresponsible individuals (Clark, 2011). Harding $(1986$, p. 82) for instance argues that, 'the addict could be cast, not as a victim of opium, but as an irresponsible individual wilfully adopting a course of self-destruction - fully aware of the probable consequences, but unable to resist the drug-induced craving'. Therefore, people who use drugs are seen as morally weak.

In addition, the moral model was incorporated in the '1930s AA 12 steps', ${ }^{14}$ which is to help people overcome addiction. The UN was also involved in developing the moral model's approach to drug use. The 1961 UN Single Convention on Narcotic Drugs - the legal bedrock of punitive approaches to drug use - refers to drug addiction as 'a serious evil for the individual' and a 'threat which the international community has a duty to combat' (Count the Costs, 2010, p. 2). Therefore, it is understandable why people who use drugs are often portrayed by the media, politicians and other important commentators as those who are morally impaired, who deny their own morality, and then become sick, promiscuous or dangerous 'outsiders' (Muncie, 2010, p. 143). Increasing surveillance and arrests of people who use drugs then become common trends among police under pressure from media and

\footnotetext{
${ }^{14}$ The 12 steps were first developed by an organisation called Alcoholics Anonymous, or AA. This mutual self-help group was founded in Akron, Ohio, in the mid-1930s. The 12 steps to overcome addiction were developed by Bill Wilson and Dr Bob Smith, who were mistakenly argued to have struggled with the disease for years before they applied the 12 steps of abstinence to treat addiction (see more at http://axistreatment.com/12-stepprograms/history/). Step No. 4 requires the person to commit to 'a searching and fearless moral inventory of ourselves' and Step No. 6 shows 'We're entirely ready to have God remove all these defects of character'.
} 
public (Muncie, 2010). Arresting people who use drugs and sending them to prison or detention centres in the name of treatment can gain strong public support.

Under the moral model, addictive behaviours are also considered as violating social rules and norms (Bauer et al., 2007). Accordingly, punishment and rehabilitation are considered to be solutions for drug addiction and are promoted as a form of help to people who use drugs, to assist them in both physical and moral recovery (Schaler, 1991). Recovery and rehabilitation consist of strengthening one's will or motivation to behave in an upright manner. Orford (2001) describes the moral model as viewing people as just needing to strengthen their willpower and to resist temptation. Yet as Buchanan (2015b) argues, people become addicted to drugs mainly because of environmental reasons and living conditions, and drug use is used as a coping mechanism in the era of neoliberalism. Specifically, Alexander (2010) explains, neoliberalism breaks the social links that give people a sense of belonging, meaning, and identity, so many people, especially people who use drugs encounter long-term dislocation from their community. As a result, dislocated people face depression, anxiety, or stress, and they take drugs to overcome these feelings and gradually drug taking becomes a coping mechanism (Peele, 2000). In addition, according to Orford (2001), the criminal justice system also approaches addiction from a moral perspective because the system intends to motivate people to behave better. Further, the criminal justice system tends to focus on those who are economically disadvantaged/powerless within societies, particularly people who use drugs. In fact, prisons are indicators of disadvantage within neoliberal systems. Nevertheless, punitive approaches only drive those who take drugs underground, further isolating them from normative society and thus exacerbating harm to users, especially from their stigma and discrimination (Global Commission on Drug Policy, 2014). Emphasising the immorality and individual weakness of spirit does not help people who use drugs to overcome their addiction.

Ironically, this moral model approach gains strong support from the general public because drug use is argued to cause addiction and crime (Akers, 1991; Walters, 1994). In other words, as shown above, there is seen to be a strong connection between drug use and addiction and crime, so implementing the moral model is seen to have dual advantages, of ensuring both justice and public order (Bauer et al., 2007; Hammersley, 2008). In Vietnam, for instance, Vietnamese people often perceive that detaining people who use drugs in compulsory 
treatment centres for physical and moral rehabilitation is a positive sign for society as it may result in a decrease in drug use, addiction and crimes (Reid \& Higgs, 2011).

The moral model of addiction is an unscientific perspective that is rooted in religion and based on classical thought, which emphasises deterrence against drug use (Clark, 2011; Ngo, 2008; Siegler \& Osmond, 1968). According to the moral model, anyone using drugs is implied to be addicted and their consumption needs to be reformed (regardless of whether they are actually addicted or not) (Siegler \& Osmond, 1968). But, as Stevens (2012) argues, forcing those who are not addicted into treatment is unethical. Further, the majority of people who use drugs are recreational users, so applying rehabilitative-based punishment upon them is generally counterproductive (Buchanan, 2015b; Levy, 2014). As Buchanan and Young (2000, p. 11) assert, punishment reinforces 'isolation and discrimination towards people who develop illicit drug problems and tends to ghettoise them within drug sub-cultures with few exit routes into 'mainstream' society'. As a result, people who use drugs find themselves trapped in a process of stigmatisation, marginalisation and social exclusion (Buchanan \& Young, 2000). For instance, many people who use drugs who are criminalised for their drug consumption encounter long-term unemployment, the restriction of social welfare like public housing, federal loans or financial aid (Levi \& Appel, 2003). These facts further marginalise and socially exclude people who use drugs from society, and inevitably exacerbate their addiction. Therefore, the moral model has little therapeutic value, and only pushes people who use drugs further away from society (Clark, 2011).

Despite these criticisms, this counter-productive moral model remains strongly influential in global drug policy. In Vietnam, for example, addiction is widely seen as a social problem and those addicted to drugs are regarded as psychologically weak people who fail to control their willpower and actions (Thien, 2002). As a result, Vietnam pursues policies that are focused on forcing people who use drugs to abstain, through the use of compulsory treatment centres where detoxification and rehabilitation changes are demanded (this will be further discussed in Chapter Five) (Windle, 2015). This moral model has, however, begun to lose influence (but the immorality of drug use still has credence) as physicians, doctors, practitioners, and policymakers have increasingly viewed addiction as a brain disease by virtue of medical breakthroughs in researching the human brain and its relationship with drug use (Wilbanks, 
1989). The moral model now combines with an alternative 'brain disease' model, to the extent that that, while addiction is now viewed as a brain disease, people who use drugs are treated as criminals and outsiders (this combination will be further illustrated below). The brain disease model has actually become a dominant model in UNODC, the US, and many other countries, including within Vietnam.

\section{Brain Disease Model}

The brain disease model was derived from a medical/disease model of addiction. The disease model looks at addiction from biological, neurological, and genetic perspectives (McLellan, Lewis, O'Brien, \& Kleber, 2000). According to this model, the use of the drug results in physical changes to the user's body and mind, which leads to significantly impaired capacity to control drug use. Accordingly, to treat addiction, people who use drugs are required to abstain from all addictive substances and activities. Nonetheless, dramatic advances during the 1970s, in both the neurosciences and behavioural sciences, revolutionised the understanding of drug addiction (Leshner, 1997). Many researchers began to reveal differences between the brains of 'addicts' and 'non-addicted' individuals, and this established the foundation for a brain disease model of addiction (Leshner, 1997). This model was first developed by Snyder and Pert at Johns Hopkins University in 1973 (Clark, 2011). They argued that biological processes are necessarily involved in addictive behaviour and that addiction is a chronic relapsing illness, characterised by compulsive drug seeking and use (Leshner, 1997; Vrecko, 2010). The brain disease model has been highly influential worldwide, from the late 1990s, after being advocated by the US National Institute on Drug Abuse (NIDA) (Trujols, 2015). This model views addiction as a consequence of a person's altered physical and psychological state after consuming drugs. In other words, chronic drug use results in dysfunction in the nervous system of 'addicts', preventing them from stopping using drugs, and leading to compulsive drug seeking and use (Leshner, 1997; Trujols, 2015).

This model tends to emphasise the significance of medicalising addiction because addiction causes a medical disorder - an abnormality of structure or function that leads to brain impairment (Gelkopf, Levitt, \& Bleich, 2002). Another perspective to explain the brain disease model is highlighted in the significance of genetics and the biological elements that shape an individual's addictive behaviour (Clark, 2011; Hammer et al., 2013; West, 2001). This means 
that brain and genetic abnormalities will significantly cause addiction. Therefore, the only way to treat addiction is to cease all drug consumption and ensure a life-long commitment to abstinence $^{15}$ (Leshner, 1997; Volkow, 2007).

Addiction treatment based on a brain disease model is a process that will not accommodate any level of drug use and it insists on abstinence and lifelong abstinence. For instance, Leshner (1997) explains that drug-taking causes fundamental changes in brain function, so a major goal of treatment must be either to reverse or to compensate for those brain changes. These goals can be accomplished through either medication and/or behavioural treatments (Leshner, 1997). Accordingly, the abstinence-based 12 steps of Narcotic Anonymous ${ }^{16}$ or the 12-step recovery programme and residential rehabilitation centres are identified as the most effective options for people who use drugs. During treatment, medication can assist clients in preventing relapse while the brain is healing and normal emotional and decision-making capacities are being restored (Volkow, Koob, \& McLellan, 2016). Also, for patients with opioiduse disorder, maintenance therapy with 'methadone'17 or buprenorphine are commonly used to help users to overcome the symptoms of withdrawal and cravings (Volkow et al., 2016). In general, the prevalence of the brain disease model is one of the key reasons for explaining why abstinence-based drug treatment remains dominant, albeit it is contested and shows little effectiveness (Clark, 2011).

The brain disease model of addiction has been globally influential. Together with the NIDA, recent American Presidents, such as Bill Clinton, George W. Bush and Barack Obama, have all endorsed the brain-disease framework at one time or another (Satel \& Lilienfeld, 2013). The

\footnotetext{
${ }^{15}$ It is important to note that Leshner was the director of NIDA, so research focusing on this model was commonly funded. Meanwhile, research that contradicts the brain disease model finds it much harder to receive funding (Hall, Carter, \& Forlini, 2015).

${ }^{16}$ Narcotic Anonymous aim to help people who have problems with drug use, through 12 steps. This model is derived from AA (Alcoholic Anonymous) programmes (see footnote 11). This approach has been adapted for the treatment of many other addictions such as CA (Cocaine Anonymous).

See more: http://www.addictionrecoveryguide.org/resources/recovery/

${ }^{17}$ After many decades of opposing substitute opioid drugs to someone heavily dependent on heroin, pragmatically in response to high fatal opioid overdose rates, the NIDA eventually altered their position and supported Methadone Maintenance. In reality, methadone is often regarded as a harm-reduction intervention, which is to mitigate harm caused by drug use, by replacing a dangerous drug with a legally prescribed substance.
} 
brain model is also a staple of medical school education and drug counsellor training. It appears in anti-drug lectures given to high-school students, and people who use drugs and 'rehab' patients are advised that they have a chronic brain disease (Koop, 2003). The model is promoted because it is believed to reduce stigma by presenting addiction as a disease rather than the result of moral failing, personal weakness or bad choices (Hammer et al., 2013). However, the model overemphasises the causes and solutions as residing with the individual. It overlooks the underlying social and psychological reasons that cause addiction, and it only increases stigma and discrimination (Satel \& Lilienfeld, 2013). In reality, addiction is more prevalent among disadvantaged, excluded and marginalised groups (Peele, 2012b). If addiction was caused by a brain disease, it is expected that addiction would be evenly spread across all sections of society.

Arguably, addiction is complex, variable, and multifactorial and it is, therefore, crucial to consider broader factors. In fact, physical changes in the brain are part of daily life: whenever people practise and do something or simply if people change their thoughts, there are changes in the brain (Satel \& Lilienfeld, 2013). Even though neuroimaging techniques can show the brain changes of people who use drugs, it is very difficult to interpret the images produced because people use many drugs in their daily routines and are affected by other confounding variables. This means that the changes detected in people who use drugs' brains are not just linked to the specific drugs that they use (Nutt, 2012). Further, as Satel and Lilienfeld (2010) argue, relying on the brain to predict or understand everything important about human subjectivity and behaviour is impossible, as it ignores the social, cultural, environmental and psychological factors that shape human temperament. The notion that addiction is defined as compulsive drug-seeking, for example, is not scientifically well demonstrated (Heyman, 2013). Many people use drugs in a controlled and relaxed manner and for those who do become addicted, most are able to regain control without professional help (Buchanan, 2008; Heyman, 2013; Levine, 2009). Arguably, if addiction is regarded as a disease, those who are addicted to drugs need to be treated with medical treatments without moral judgement and in the same manner like other diseases.

However, as Trujols (2015) argues, regarding addiction as a pathology of the 'weak-brained' or 'weak-gene bearers' only promotes stigma and creates marginalised groups. As a consequence, people who use drugs cannot choose, control their lives, or be held accountable 
for their addiction; instead, they are required to attend abstinence therapies to avoid relapse, which can lead to more social isolation and stigma. Similarly, Hart (2017) states that viewing addiction as a brain disease promotes societal injustice. He explains that if the real problem with drug addiction is the interaction between drugs and people's brains, then the sole solution for this would be removing drugs entirely from society via law enforcement measures. As a result of this common thinking, people who use drugs are commonly incarcerated in prison or compulsory treatment centres. However, a study conducted by Tomori et al. (2014) show that many people who participated in abstinence-based treatment in compulsory treatment centres have reported that they find it difficult to re-integrate back into society, find work and access other social and health-care services because of being labelled as compulsory treatment centre inmates. Although the disease model does not try to blame people who use drugs, as it focuses on factors beyond the control of users such as family history, genetics, and biological vulnerability, there is still a coercive element to treatments and official responses (Marlatt \& Witkiewitz, 2010). Arguably, this illustrates that the disease model is a layer on top of the moral failing model, so the moral judgements are still existing and are embedded within the brain disease model.

As the brain model tends to focus on human brain solutions and medical interventions, it overlooks the person-behaviour-environment interactions or set and settings of drug use as central concerns of addiction (Buchanan, 2004b, 2015b; Clark, 2011; Peele, 2012a; Rastegar \& Fingerhood, 2005; Uchtenhagen, 2010). Nonetheless, this approach has been favoured by many international organisations and UN agencies. The World Health Organization (WHO (2003, p. 7), for instance, states that:

Addiction is a chronic relapsing disorder with a biological and genetic basis, and is not simply due to a lack of will or desire to quit ... [it] is as much a disorder of the brain as any neurological or psychiatric illness.

In recent years, the brain disease model has faced criticisms. It has been argued that this model is lacking in considering genetic, environmental, and developmental factors when it comes to addiction (Becoña, 2018). Further, many scholars have proposed that addiction has, in part, a neurobiological basis (along with relevant psychological and social factors) (Hall et al., 2015). In other words, genetic/biological, psychological, and sociocultural factors are 
important for developing an addiction (Becoña, 2018). For example, Farisco, Evers, and Changeux (2018) argue that the neurochemical bases of consciousness influence addiction, and socio-economic and ecological contexts have a significant impact on brain processes. They deem that the brain and its living contexts have a strong connection that affects how people are addicted to drugs. As a result, addiction is seen as a medical and mental disorder, as well as a social disorder (Farisco et al., 2018), so treatment still requires a reduction in drug intake or lifelong abstinence, which is not always a preferred option among many drug users.

However, in reality, many countries, Vietnam included, have been strongly influenced by the Joint United Nations Programme on HIV/AIDS (UNAIDS) and WHO, which view addiction as a brain disease. Therefore, Vietnamese government documents and legislation endorse the view that addiction is a brain disease (Ahmed, Long, Huong, \& Stewart, 2014; Ha et al., 2010; Khuat et al., 2012; Nguyen \& Scannapieco, 2008; Peele, 2012a; The National Committee on AIDS Drugs and Prostitution Prevention and Control, 2011; Thien, 2002). For example, according to the 2006 HIV Prevention Law, addiction is a brain disease that is naturally chronic and relapsing, which is characterised by compulsive drug seeking and use, despite harmful consequences (VAAC, 2011). In general, with the strong support of UN agencies as well as US political leaders, the brain disease model, which promotes an abstinence-based approach to address drug use and treat addiction, has been dominant worldwide, Vietnam included. Although the brain disease model has become dominant, it is not to say that approaches that conform to the moral model have disappeared. Actually, these addiction models exist simultaneously and establish a strong foundation for the prevalence of abstinence-oriented responses or prohibition to drug use in many countries (Clark, 2011), Vietnam included. This narrow and problematic view of addiction is what informs responses to drug users in Vietnam, leading to the prevalence of 06 centres and of punitive approaches. Nevertheless, as noted above, drug addiction is a complex and contested concept and it is influenced by wider social and psychological factors (Hammer et al., 2013). Therefore, a psychosocial model of addiction should be considered.

\section{Psycho-social Model of Addiction}

Since the end of the 1970s, psychological and sociological factors of individual addiction have been more widely considered (Clark, 2011). According to Hendin (1980), the interrelation 
between social and psychological variables are important for producing adaptive and maladaptive behaviours among people who use drugs. Also, Peele (2000) claims that people who use drugs learn to be addicted through observed and learned behaviour or subjective experience. He argues that individual, situational or cultural outlooks, alongside cognitive factors, are essential in shaping addiction behaviour. In fact, people take drugs because they want to acquire certain desired results, such as coping with stressful or aversive environments, reducing depression, gaining pleasure, or relieving emotional, physical and psychological trauma (Peele, 2000). In contrast to the moral and brain disease model of addiction, which argues that addiction is a problem caused by addictive drugs, social and psychological models of addiction posit that addiction is a problem caused by wider social and environmental factors (Buchanan, 2015b). Bruce Alexander's Rat Park, and work by Zinberg (1986), as noted in Chapter Two, ${ }^{18}$ demonstrates that the setting is more important than the substance or the personality of the user. Their work undermines the moral and brain disease models that see set and substance as the vital components of addiction.

Similarly, Gabor (2010) argues that addiction is influenced by the environment in which people live and interact. He claims that many problematic drug users face an inner emptiness, which is caused by societal dislocation, such as broken relationships within the family and the community, childhood trauma, social abandonment, abuse or a lack of communication between parents and their children. As a result, addiction is not the primary problem; rather addictive drug use is a response to soothe these pains. In other words, people become dependent on drugs because they want to relieve their social and psychological problems such as anxiety, depression, fear, poverty, exclusion, abuse, dislocation and/or hopelessness, which treatment and coercive treatment do not address (Buchanan, 2004b, 2015b; Peele, 2012a; Rastegar \& Fingerhood, 2005; Uchtenhagen, 2010). Therefore, many people who use drugs find themselves unable to overcome addiction because of the debilitating impact of their previous experiences, or because of their poverty, social discrimination, stigma, or sense of alienation within the community (Lewis, 2015). These strong associations between

\footnotetext{
${ }^{18}$ Alexander and Zinberg both confirm that arguing for addiction as a problem caused by an addictive drug or a person is far too simple. They demonstrate that the majority of people become addicted to drugs because of the setting. Users take drugs as a mechanism to address their social and environmental issues.
} 
addiction and the setting continue to cast serious doubt on brain disease and a moral failure in relation to addiction, which highlights the role of the drug and the set.

Nevertheless, not all countries view addiction from psychosocial perspectives. Vietnam is a typical example. In Vietnam, laws and government documents prioritise the moral and brain disease models of addiction and insist on abstinence. As a result, practical interventions mainly focus on protecting the wider community by law enforcement and disregard the importance of addressing societal issues associated with people who use drugs like social isolation, stigma, discrimination, or human rights (Edington, 2016). In other words, abstinence is seen as the key method for addressing drug use related problems in Vietnam. The next section explores key components of an abstinence-based approach, its responses to drug use, and the issues surrounding this concept.

\section{Abstinence}

As noted above, abstinence approaches have been heavily shaped and influenced by prohibition or the 'Drug Wars' that outlawed and demonised particular substances as dangerous. Therefore, any philosophy or treatment of people who used them had to be built around abstinence. Also, as noted above, due to the prevalence of the moral and brain disease models of addiction, abstinence is regarded by many governments as the most effective means of overcoming addiction and preventing drug-related harm (Dolan et al., 2007; Wodak, 2011). People who use drugs are argued to have lost control over their drug use behaviour (Goldberg, 1999). In response, people see that the only hope to overcome addiction and regain control is to stay completely away from illegal drugs and ensure longterm abstinence (Buchanan, 2008; Thompson, 2007).

This section illustrates key components of abstinence in regard to general philosophy, responses to drug use, and relationships of power. The key arguments of abstinence-based approaches are that illicit drug use is harmful, causing addiction and many social problems, so it is unacceptable in society. Responses to drug use are therefore based upon prohibition, criminalisation and a long-term period of forced abstinence and rehabilitation. In addition, people who use drugs generally have no role to play in addressing their problems because they are seen as having lost control. This section argues that abstinence is an impractical 
approach because it purposely seeks the elimination of all illicit drug use from society (Wodak, 2011). Also, by imposing unrealistic and unachievable expectations upon all people who use drugs, abstinence-based approaches generally have made some users more damaged, isolated and at risk (Global Commission on Drug Policy, 2014).

\section{General Philosophy}

Abstinence refers to approaches that aim to prevent all illicit drug use and help people stop using drugs for the rest of their lives (Chang \& Lin, 2015). Any approach to drug problems, therefore, should not accommodate ongoing drug use. Currently, due to the prominence of the moral and brain disease theory of addictions, total lifelong abstinence and recovery are seen to be required for the purpose of eliminating all drug use from society (Goldberg, 1999). Abstentionists tend to assert that people who use drugs have low moral standards, make bad choices for their lives and are genetically/biologically weak, so people who use drugs are labelled as threats to society, 'addicts', criminals and diseased persons (Stevens et al., 2010). They also assume that all drug use is bad and that use inevitably leads to addiction (Buchanan, 2006). Importantly, abstentionists believe that abstinence and prohibition can reduce drug consumption (Hilton, Thompson, Moore-Dempsey, \& Janzen, 2001). By increasing punishment, employing tougher law enforcement and treatment (including compulsory treatment), abstentionists deem that they can mitigate the harms caused by drugs (Levy, 2014).

Abstinence seeks to reduce drug-related crimes (such as crimes committed while someone is intoxicated, or crimes to fund a drug habit) by destroying the relationship between drugs and crimes (Holloway, Bennett, \& Farrington, 2005). Therefore, the overarching goal of abstinence is to quit drug use and to keep people who use drugs 'clean' to stave off the 'disease' of addiction as well as promoting life choices that never involve drug use in order to reduce drug-related crime (Buchanan, 2008; Goldberg, 1999). While it should be acknowledged that drug addiction needs to be addressed and reduced, addiction only affects a small percentage of people who use drugs (the rest use drugs in a controlled or recreational manner).

In fact, many studies show that despite the growth of abstinence-based approaches and strategies, drug use and drug crimes have not declined (Levy, 2014). For example, according to a recent UNODC (2016b) report, there was a significant increase in the number of people 
using illicit drugs worldwide. There were about 180 million people using illicit drugs in 1998 and the number increased to nearly 203 million in 2008, while the estimated figure for 2015 was around 255 million people. Apparently, an ever-increasing number of people continue to use illegal drugs despite the great efforts to tighten abstinence-based strategies such as prohibition, criminalisation or punishment. For instance, according to UNODC (2016b), from 2005 to 2014, the amount of cannabis and synthetic drugs seized by police of UN member states increased year after year, while other drugs like opioids, heroin or cocaine did not show any downward trend. Similarly, although the Vietnam government further promoted and strengthened their drug law enforcement, the number of drug cases detected and prosecuted by police still increased between 2011 to 2014 (MPS, 2015). In general, as Hughes and Stevens (2010) indicated, tightening up on abstinence-based approaches (like demanding harsher punishment) has limited effect on the rates of drug use, and people still continue using drugs for different reasons. Yet these 'drug-free' approaches have still dominated worldwide when it comes to responding to drug use problems.

\section{Responses to Drug Use}

As detailed above, illicit drug use is commonly argued to be harmful, causing crime and addiction, so corresponding responses to people who use drugs are usually punitive and based on abstinence. These responses are influenced by many factors rather than evidence, science or rationality (Stevens \& Measham, 2014). As Hutton (2016) argues, societal and official responses to drugs and people who use drugs among abstentionists are often based on fears about particular groups. The history of drug control demonstrates why punitive drug controls were introduced in response to fears about particular groups. For instance, the use of punitive legislation in New Zealand in the 1900s was aimed mainly at the Chinese ${ }^{19}$ communities who were blamed for bringing opium into New Zealand and spreading it throughout the country (Eldred-Grigg, 1984). A similar situation also took place in the USA. At the beginning of the $20^{\text {th }}$ century, a punitive drug policy was established to control and punish

19 China tried to control its commercial markets from the exploitation of the British, particularly opium in the early 1800s (Carter, 1990). Unfortunately, Britain refused to stop trading opium, so China fought, but it lost. Later, Britain took Hong Kong as a result of the Chinese failure. 
Chinese people who use drugs and Chinese opium farmers (Epstein, 1990), while the control of marijuana and cocaine consumption was often associated with Mexicans and Black Americans respectively (Hutton, 2016). Similarly, in Vietnam, drugs like opium or cannabis were first introduced in the $17^{\text {th }}$ century, and initial drug control policies were also targeted at opium and cannabis users (Duc, 2012). The key assumption for targeting those people was that, as noted previously, they were perceived as a threat to society; they were presented as an enemy of society, and the cause of community despair $(\mathrm{Vu}, 2000)$. By punishing those particular groups, the authorities believed that drug use would be curbed and society would be protected. Yet these government's arguments were racist and designed to exclude these particular groups (people who use drugs) rather than curbing drugs.

Fears about particular groups have often been reinforced by sensationalised media coverage, which has a strong influence on drug policymakers (Coomber et al., 2013; Hutton, 2017; Taylor et al., 2016). In reality, news media and governmental beliefs mirror each other and both adopt the common perception that drug use is dangerous (Taylor, 2008). For example, it is common to see dubious claims in news media that 'cannabis use leads to psychosis and violence, or crack cocaine taken during pregnancy led to mentally damaged babies that would struggle to function normally' (Buchanan, 2015b, p. 62). Accordingly, the negative and stereotypical representations of those who take drugs as criminal outsiders and a threat to society are also normalised (Taylor, 2008). As Boland (2008) notes:

These views dominate media coverage and are embedded in the public mind-set to the extent that illegal drugs users are treated like social pariahs and blamed for all manner of today's social ills (Boland, 2008, p. 173).

This is also the case in Vietnam where media (controlled by government) and the government often emphasise that drug use and users cause social disorder and crime. For instance, ANTV, the most popular television channel of Vietnam, which focuses on social order and public security, often bombards people with sensational headlines like 'drug use and addiction is the shortest path leading to criminal behaviours' (Dinh \& Duc, 2014, p. 1). This reinforces the common mind-set that there is an inevitable link between drug use and criminal behaviour, and this justifies and sustains the use of punitive responses to drug use and people who use drugs within Vietnamese society. However, the fears of particular social groups are arguably 
based on myths and misconceptions rather than evidence, and these fears are driven by a political purpose (Chomsky, 1998; Coomber et al., 2016; Coomber et al., 2013). As detailed earlier, the majority of people who use drugs are recreational users. Nevertheless, as Buchanan (2015b, p. 59) explains, many governments still conduct punitive responses to drug use because:

It is an effort to stimulate fear of dangerous people from who we have to protect ourselves. It is also a direct form of control of what are called "dangerous classes," those superfluous people who don't really have a function contributing to profitmaking and wealth. They have to be somehow taken care of.

In considering the widespread abstinence and punitive approaches to drug use and people who use drugs, the concept of 'moral populism' is significant within this debate (Hutton, 2017). This concept partly refers to the idea that drug policies are wedded to outdated notions of both drug-related harm and people who use drugs (Hutton, 2016), a point discussed above in relation to UN Conventions. These Conventions, as Nutt, King, and Philips (2010) argue, are outdated and ignore the overwhelming evidence in relation to the benefits of drugs. For example, opium can be used for severe pain management, while cannabis and MDMA can support the treatment of epilepsy or Post-Traumatic Stress Disorder (Buchanan, 2016a). One of the reasons for the dominant influence of 'moral populism' is that the rise of populist politics has arguably influenced political agendas (Hutton, 2017). For instance, Bottoms (1995, p. 40) explains that politicians tend to prefer punitive approaches because many of them believe that they will be popular with 'the voting public'.

Importantly, as Chermak (1997) argues, raising fears of particular social groups is seen as a tool that many politicians, police or prosecutors use to receive public attention and create political success. Punitive populism is therefore seen as one of the key drivers of drug policymaking and of increasingly harsh punishments towards drug use and people who use drugs in recent times (Wood, 2014). This is also the case in Vietnam. For example, a study conducted by Edington and Bayer (2013) on Vietnamese drug policy revealed that many politicians gain popular support by targeting people who use drugs and getting users off the street (further detailed below). As a result of the misperceptions and fears of those who taking illicit drugs, abstentionists tend to place an emphasis on controlling people who use drugs. 
In order to control people who use drugs, abstentionists distinguish between legal and illegal drugs and engage in arguments that illegal drugs lead to addiction, health and crime problems. By separating legal and illegal drugs, abstentionists believe that they can control those taking illicit drugs and that, by doing so, the harms caused by illicit drugs will be reduced (Goldberg, 1999). Accordingly, abstentionists tend not to differentiate between different types of illegal people who use drugs, meaning that experimental or recreational users who may use drugs sensibly or responsibly are regarded as the same as problematic users who struggle with addiction or cause harm to themselves or others (Edington \& Bayer, 2013). In addition, abstentionists often argue that punishing people who use drugs can stimulate fear of taking drugs in other people (Chomsky, 1998). Through this general deterrence, the number of new people who use drugs can be reduced, leading to the overall reduction of drug consumption. Yet as noted previously, punishment has no effects on drug use. Ironically, in Vietnam, governments and the general public often believe that punishing those who take drugs and sending them to coercive treatment can reduce the crime rate and ensure social order and public security (Windle, 2015).

A common method to control drug use and people who use drugs is to implement coerced abstinence interventions or compulsory addiction treatment (Hilton et al., 2001; Thompson, 2007). This approach is based on the common belief among abstentionists that 'once an addict, always an addict' (Satel \& Lilienfeld, 2013, p. 1). In Asian countries like China, Malaysia, Laos, the Philippines, Thailand, Cambodia and Vietnam, the governments believe that compulsory treatment centres assist people who use drugs in giving up their drug use and helping them to recover (Bean 2014). According to UNAIDS (2016), about 456,000 addicts were detained in compulsory treatment centres in those countries in 2014. In the centres, people who use drugs generally have to comply with the 12 steps of Narcotic Anonymous or the 12-step recovery programme to stave off their addiction and regain control (Borkman, Kaskutas, \& Owen, 2007; Lewis, 2015; Peele, 2012a). Importantly, the UNODC (2010b, p1) also states that 'coerced drug treatment is an acceptable alternative to imprisonment that is consistent with international drug control treaties and supported by evidence of effectiveness'. This narrative has encouraged member states to expand the system of drug courts, drug testing and compulsory treatment centres. Yet as noted previously pertaining to 
populism, governments mainly use 'drug courts', 20 'drug testing', ${ }^{21}$ and especially compulsory treatment as a tool to control specific social groups (people who use drugs) rather than providing them with necessary assistance.

People who use drugs are forced into treatment simply because they use drugs, regardless of the nature of their use. Further, recreational drug use and other ways of using drugs are not recognised, and use is widely equated with misuse or addiction. Detaining different types of people who use drugs together into treatment centres, without dealing with their drug use situation, is a further key reason why such treatment is often ineffective. This treatment approach focuses on individualising and medicalising addiction, emphasising factors like genetics or biological vulnerability (Clark, 2011). Therefore, this coercive approach overlooks social, psychological or environmental factors, which are far more important in shaping and developing addictive behaviours (Clark, 2011; Satel, 1999). This also overlooks the fact that many of those detained are recreational users who do not need treatment.

\section{Abstinence and Relationships of Power}

In coercive treatment, governments tend to overlook the heterogeneous nature of drug use and generally deny people who use drugs the opportunity to make decisions for themselves about how they might overcome their drug use problems. In addition, as noted previously, people who use drugs need to surrender their addiction, accept that they are powerless over their addiction and, importantly, need to 'hit the bottom'22 before they are ready to be provided with help (Carr, 2010). As a result, users are deemed to have no rights to make decisions for their own sake, especially with regards to options for treatment. In fact, clinicians will play an 'expert' role in resolving problems by telling users what to do (Miller \& Rollnick, 2012). In treatment, any 'relapse' following interventions is a sign of individual failure

\footnotetext{
${ }^{20}$ Drug courts are a process in which people who use drugs are placed into abstinence-based treatment and proactively monitored by the judge, the criminal justice system and treatment professionals with the aim of assisting the people who use drugs to stop using drugs. These courts are a popular abstinence-based approach that involve frequent and random supervised drug testing (Weatherburn, Jones, Snowball, \& Hua 2008).

${ }^{21}$ Drug testing aims to identify who people who use drugs are, in order to create a drug-free environment (Maltby, 2001). It is conducted via urine, blood, hair, fingers or sweat in order to find out those who take illicit drugs.

${ }^{22}$ This is also called 'tough love approach', which justifies inhumane responses.
} 
to conform to 'treatment', and abstentionists deem that compulsory treatment is the only option for the majority of people who use drugs (MacMaster, 2004).

People who use drugs are seen as being incapable of deciding their treatment because, as mentioned earlier, from an abstinence perspective (which emphasises neurological, genetic, biological or moral factors in shaping addiction), people who use drugs are perceived as having lost control over their drug-taking behaviour. As such, people who use drugs need to follow the instructions of service providers to abstain from drugs. According to the 12-step recovery programme, people who use drugs are detoxified and if necessary provided with medical assistance to overcome addiction, and they are then re-educated morally to become a 'normal' person again (Kamarulzaman \& McBrayer, 2015). Also, compulsory exercise and/or labour therapies are common elements of 'treatment', either in the belief that they will help detainees to overcome drug dependency or to aid in social rehabilitation upon release (Human Rights Watch, 2012). Yet abstinence-based approaches, such as forcing people who use drugs to follow treatment and denying their rights, are ineffective, as discussed next.

\section{Counterproductive Effects of Enforced Abstinence}

While abstinence-based approaches have emerged as a dominant global drug policy since the early $20^{\text {th }}$ century, the coercive approach, the mass criminalisation of people who use drugs and punitive laws have arguably caused many harms and negative consequences to people who use drugs (Global Commission on Drug Policy, 2014; Trace, Somogyi, Hallam, \& BewleyTaylor, 2007). Regarding the coercive approach, the WHO (2009) and Human Rights Watch (2011) have disputed the effectiveness of this treatment method and outlined their widespread ethical problems. According to Werb et al. (2016), who conducted a systematic review of the effectiveness of compulsory drug treatment, the majority of studies (78\%) show that compulsory treatment fails to foster any significant positive impacts on drug use or criminal recidivism. Many studies have revealed that coercive treatment is expensive and not cost effective, while relapse rates are high (Birgin, 2013; Seddon, 2007; Stevens, 2012; Stevens et al., 2010). For example, in the USA, Satel (1999) indicates that about half of compulsorily treated people who use drugs relapsed in the first three months, while the rate of relapse by the end of the first year was 80 to $90 \%$. Coercive approaches have also been criticised for ethical problems, such as human rights violations and having a high level of 
physical violence and torture (Human Rights Watch, 2011, 2012; International Labour Rights Forum, 2014; Vuong et al., 2017). Many people who use drugs were infected with bloodborne diseases, such as HIV or hepatitis B after entering compulsory treatment centres (Human Rights Watch, 2011; Wodak, 1998). In such circumstances, forcing people who use drugs into treatment becomes counterproductive and dangerous.

The mass criminalisation of people who use drugs and overly punitive laws have arguably caused stigma and discrimination towards people who use drugs rather than providing help (GCDP, 2014; Trace, Somogyi, Hallam, \& Bewley-Taylor, 2007). For instance, a study conducted by Nutt and colleagues in 2010 revealed that the harms and deaths associated with the use of heroin and ecstasy (Class A drugs) largely derive from criminalisation and black market production and provision rather than the drugs themselves. Similarly, a study by Hargraves (2015, p. 3) demonstrated that injecting people who use drugs often experience stigma and discrimination, they are not considered a part of 'normal' society and are commonly branded as being deviants, diseased, unemployed, uneducated, and criminals'. The key stigmatisers of people who use drugs are not only from their communities, but ironically from service providers that adhere to abstinence/moral models of drug use and treatment. Also, according to this study, experiencing stigma and discrimination limits the ability of people who use drugs to find work or hold down a job, participate in day-to-day activities, and their willingness to access relevant services like health services (Hargraves, 2015). In other words, punitive drug policy drives those who take drugs underground, further isolates them from normative society, reduces opportunities for education, outreach and employment, thus again exacerbating harm to people who use drugs, especially stigma and discrimination (Global Commission on Drug Policy, 2014).

Punitive approaches have caused stigmatisation of and discrimination against people who use drugs. For instance, in Vietnam, Vietnamese people who use drugs were widely condemned for causing the twin epidemics of heroin injecting and HIV infection in the country (Cohen, 2003; Le, Ngoc, Hoang, Mulvey, \& Rawson, 2013). Since the 1990s, the Vietnamese government has launched intensive campaigns against 'social evils' with public propaganda campaigns against people who use drugs who are portrayed as weak-willed and morally corrupted individuals, as blights on the honour of their family and the country as the whole 
(Edington, 2016). However, discriminating and stigmatising against people who use drugs did not help Vietnam's government and society to diminish drug use or HIV infection, as the number of people who use drugs and HIV infected people have increased year by year since the launch of the campaign (Windle, 2012). As such, it is essential to have rational responses to drug use and people who use drugs in order to not only address health-related harms caused by drug use, but also, importantly, to focus on the harms to people who use drugs that arise out of the legal framework for drug prohibition, criminalisation and the consequences of such punitive approaches (Hunt et al., 2003).

\section{Conclusion}

It is evident that abstinence-based approaches focus on the perception that drug use and addiction is a moral problem or a brain disease. Therefore, abstentionists do not accommodate ongoing drug use and aim to persuade people to stop using drugs and endeavour to prevent drug use. Abstinence is preferred by most governments and policymakers because it is thought to reduce drug consumption, drug crimes and mitigate the harms caused by drugs. Further, under a framework of 'moral populism', UN conventions on illicit drugs and national laws rooted in prohibition, abstentionists often regard and categorise people who use drugs as dangerous, morally reprehensible and diseased persons who are in need of harsh punishment and control. These representations of people who use drugs are to serve political purposes rather than evidence, but they remain widespread (Buchanan, 2016a). Also, given these assumptions, it is commonly regarded that people who use drugs cannot make appropriate decisions to address their addiction problems. In turn, drug punishment and coercive drug treatments are identified as key methods for helping them and preventing harm caused by drug addiction and for protecting people from the dangers of drugs and drug addiction. Yet by enforcing unrealistic and unachievable expectations upon people who use drugs who are expected to stay away from all drugs, enforced abstinencebased interventions like prohibition, punishment and coercive drug treatment have made users more damaged and isolated (Buchanan, 2015b).

Although enforced abstinence approaches have caused many problems to people who use drugs and struggled to achieve their goal, in countries like Vietnam where drug use is historically and socially regarded as a social evil, such approaches remain dominant. These 
approaches have been shaped by UN Conventions that have emphasised the dangers of all illicit drug use and the legitimacy of stringent responses. As such, UN member states tend to carry out punitive approaches to drug use and, following the lead of the USA and allies, have also accepted prohibition (Levine, 2002, p. 168). Drug prohibition has also served other political purposes (Levine, 2002), not least as it can be politically useful to identify an 'enemy' to control. This is evident within Vietnam, where the government has developed power by controlling drug use and people who use drugs (Chapters Four and Five will further examine this point).

Government responses to drug use, addiction and people who use drugs tend to emphasise individualised medical solutions, generally ignoring the complexities of drug use behaviours and the intersections between drug use with social and environmental factors, as highlighted by the psychosocial model of addiction (Alexander, 2010; Peele, 2012b). Therefore, a comprehensive response to drug use and addiction must take into account both the human rights of drug-dependent individuals and those of the people who live in their communities (Wu, 2013). While abstinence-based approaches seek to protect society from harms caused by illicit drugs, the evidence indicates that such approaches can cause more harm and offer little or limited benefit to either people who use drugs and non-users (Taylor et al., 2016). Current responses to drugs are driven more by flawed assumptions and political populism, rather than evidence, science and rationality (Coomber et al., 2013; Hutton, 2017; Taylor et al., 2016). Therefore, abstinence approaches fail to tackle fundamental drug use problems, particularly how and why people become addicted to the drug and how to help them overcome addiction.

Harm reduction is seen as a more effective approach to address the complexity of drug use behaviours and meet the needs of people who use drugs (Ritter et al., 2011). Harm reduction refers to an approach that aims to reduce the adverse health, social and economic consequences of illegal drug use without necessarily reducing drug consumption (Riley et al., 1999). Despite some critiques (largely due to its challenge to abstinence), harm reduction is seen as an effective and pragmatic response to drug use with fewer side effects (Borsos, 2009; Peele, 2012b). The next chapter will critically explore how harm reduction has developed, including its philosophy, and then demonstrate why it should be a global response to drug use and addiction. 


\section{Chapter Three: From Abstinence to Harm Reduction}

\section{Introduction}

Globally, current responses to drugs are driven more by abstentionist myths, flawed assumptions and political populism, rather than evidence, science and rationality (Coomber et al., 2013; Hutton, 2017; Taylor et al., 2016). Harm reduction, an approach that emerged in its contemporary form from the 1980s when the HIV/AIDS epidemic became a serious problem, is seen as an effective approach to address the complexity of drug-use behaviours and meet the needs of people who use drugs (Ritter \& Cameron, 2006). Harm reduction has also shifted the way people and governments react to drug addiction and drug use related problems (Harris, 2012).

For example, from the mid-1980s, the UK, Dutch and Swiss governments realised that the spread of HIV infection among people who use drugs was a greater danger to individual and public health than drug misuse (McKeganey, 2011). The HIV epidemic not only affected people who inject drugs, but also non-people who inject drugs and the wider community, so the focus of these governments shifted from seeing drug use as a criminal problem to seeing it as a public health threat (Collins et al., 2012; Hilton et al., 2001). It is worth mentioning that harm reduction was implemented in the form of needle exchanges to stop the spread of HIV, rather than in consideration of the welfare/wellbeing of people who use drugs. Similarly, from the 1990s the main UN organisations, (such as WHO, UNODC and UNAIDS) have adopted harm reduction in their activities; however, these harm reduction interventions have primarily focused on public health issues like HIV prevention, opioid substitution therapy for drug addiction or overdose prevention (UNAIDS, 2016).

Nevertheless, harm reduction is more than just a medical intervention as it importantly promotes human rights and drug policy reform (International Harm Reduction Association, 2016). Harm reduction has been viewed as a critique of the deficiencies of prohibition and abstinence, as researchers have argued that prohibitionist policies threaten the freedom of people who use drugs, damage their health and constitute them as marginal and stigmatised groups (Elliott, Csete, Wood, \& Kerr, 2005). With many benefits, such as reducing HIV infection rates, promoting human rights, reducing stigma or crimes, harm reduction has been 
seen as an alternative paradigm of dealing with both problematic and recreational drug use (Harris \& McElrath, 2012; Roy \& Buchanan, 2015).

This chapter starts with a brief history of harm reduction, which paves the way for understanding how harm reduction has been implemented and why it has had a narrow public health perspective. Next, the chapter points out how harm reduction has been defined from many perspectives. There is still no formalised, internationally agreed definition concerning the scope of harm reduction, but the cornerstones of harm reduction in this study are public health, human rights and social justice. To illustrate these cornerstones, the chapter analyses key principles of harm reduction (how drug use is conceptualised, how people should respond to drug use, and relationships of power). From here, it further explains why harm reduction should go beyond a public health perspective. Despite some ongoing challenges, harm reduction demonstrates an effective response to drug use, and it should be considered an alternative approach to enforced abstinence (International Harm Reduction Association, 2016).

\section{Brief History of Harm Reduction}

Harm reduction started in Britain. In the $19^{\text {th }}$ century, the goal of reducing drug-related harm became an important element of drug control there (Berridge, 1999). Incorporating public health concerns and medical expertise, the country legitimised the prescription of maintenance doses of drugs as a form of reducing drug-related harm such as providing prescriptions of pharmaceutical grade heroin for heavily dependent heroin users (Berridge, 2009). This practice was intended to avoid serious withdrawal symptoms and help patients have a useful life (Harris, 2012). Much later, in the 1960s, drug clinics and centres in London started to guide people who use drugs on how to inject drugs safely (Richard \& Riley, 2012; Riley \& O'Hare, 2000). However, it was not until the 1980 s that harm reduction began to develop into a more formal and coherent approach in the wake of the HIV epidemic (Marshall, Dechman, Minichiello, Alcock, \& Harris, 2015).

The emergence and spread of HIV infection, especially among people who inject drugs, prompted public health sectors around the world to promote HIV prevention interventions (Riley et al., 1999). This embodiment/enactment of harm reduction, therefore, focused 
primarily on drug injection and blood-borne diseases. The Netherlands, for example, adopted a harm reduction public policy in the 1980s, which allowed health service centres to provide sterile needles and syringes directed for heroin injectors (Marlatt, 2000). Importantly, this country began to apply a 'low-threshold approach'23 to people who use drugs, which then became one of the key principles of harm reduction strategies worldwide. Adopting this Dutch approach, in 1986, public health workers and government officials in Liverpool, England, started a syringe programme to curb HIV (O'Hare, Newcombe, \& Matthews, 1992). This programme led to the birth of other types of harm reduction interventions, such as the provision of clean injection equipment, the maintenance prescription of opioids or methadone maintenance treatment (Doherty et al., 2000). As a result, HIV infection was controlled and the greater number of people who use drugs engaged in harm reduction services. This developing practice led to the first international harm reduction conference in 1990, and since then harm reduction has become a global movement (Roe, 2005).

Harm reduction interventions now operate in a wide range of social, cultural and religious settings across Asia, the Middle East and North Africa (Kamarulzaman \& Saifuddeen, 2010). Indeed, according to Harm Reduction International (HRI, 2016) harm reduction programmes currently operate, at some levels, in more than half of the 158 countries in the world where injecting drug use has been recorded. Today, harm reduction does not only focus on injecting or problematic drug users, but it is a philosophy that can be applied to diverse ways of using drugs (e.g. recreational/experimental) as well as legal drugs like alcohol. In fact, harm reduction continues to demonstrate its effectiveness in addressing HIV transmission, viral hepatitis, and overdose, and, importantly, in promoting health, human rights and dignity towards people who use drugs worldwide (Harm Reduction International, 2016). Harm reduction is thus seen as a pragmatic approach to drug use rooted in science, public health, human rights and dignity. Although harm reduction is generally effective as argued above, its definition, philosophy and practice are still debated.

23 This approach entails having few or no conditions on users to access services. In other words, harm-reduction services are open for all people who use drugs, both recreational and problematic. 


\section{Defining Harm Reduction}

While the contemporary concept of harm reduction was developed in the 1980 s, as noted above, there is still no formalised, internationally agreed definition concerning the scope of harm reduction (Collins et al., 2012; Parkin, 2014; Riley et al., 1999; Stevens et al., 2010). Initially, in the wake of the HIV epidemic in the 1980s, harm reduction philosophy involved pragmatic strategies towards intravenous people who use drugs, solely to reduce the risk of HIV to the non-drug using population (Riley \& O'Hare, 2000). Given the diversity of harms that result from drug use, Doherty et al. (2000) and Nutt (2012) argue that other types of harm upon the individual, community and society should also be addressed. While many define harm reduction as an approach that does not exclude abstinence-based treatment for those people who use drugs seeking it, some suggest that abstention should not be the ultimate goal of harm reduction (Lenton \& Single, 1998).

Harm reduction is different from abstinence in its emphases, but it recognises that abstinence may be either a realistic or/and desirable goal for some (Marlatt, 2000). In fact, harm reduction neither precludes nor discourages abstinence-based goals as long as they are generated by and acceptable to the affected individual and do not cause more harm (Denning, 2001). This means that harm reduction can support abstinence if clients want to do so. In other words, harm reduction approaches may promote use reduction and even support abstinence when still providing other empirical support beneficial to the individual, community and society, such as housing, employment or education (Collins et al., 2012).

Furthermore, Stimson (2007), Stafford (2007), and Tammi and Hurme (2007) define harm reduction by emphasising its three pillars. First, illicit drug use should be viewed neutrally, not morally (non-judgementalism). As discussed previously, all legal and illegal drugs can be harmful, but not all drug use is problematic. It has been demonstrated by the UNODC (2017) that only one out of ten people who use drugs has problems with their drug use. Therefore, illicit drug use should be seen from a neutral perspective like other legal drugs. Second, drug policy needs to be pragmatic, and based on scientific evidence, not on ideological beliefs or dogmatism. That is, practical approaches need to be specific and targeted at realistic goals, such as reducing HIV infection, stigma, discrimination or social disadvantages for people who use drugs, rather than the pursuit of unrealistic goals, like a 'drug-free community'. Third, 
drug policy should respect the legitimate rights of people who use drugs, and facilitate noncoercive and non-punitive approaches. This pillar of harm reduction attacks punitive prohibition or abstinence-based approaches, which are often seen as the greatest source of harm related to drug use, as discussed previously. These pillars of harm reduction are important to guide practice. Yet given the prevalence of abstinence-based mind-sets among policy-makers, harm reduction is still misperceived by many governments.

\section{'Pseudo' Harm Reduction}

Weatherburn (2009) explained that from many governments' and policy-makers' perspectives, harm reduction is often misguidedly seen as any programme and policy that is intended to reduce the harm associated with drug use and problem behaviour. From this perspective, as Riley et al. (1999) and Ritter et al. (2011) explain, governments and leaders tend to pursue abstinence or the imprisonment of people who use drugs for drug possession because they think it can reduce the harms caused by drug use. They view such actions as a form of harm reduction, but I would call it 'pseudo' harm reduction. This misperception is common, but dangerous because it leads to counterproductive ideas. As Stevens et al. (2010) argue, many policy-makers imagine the best way to reduce drug-related harm is through the criminal justice system, by reducing the number of people who use drugs in society. For example, in the United States, Single (cited in Hathaway 2001), explains:

The harm reduction movement is in sort of an identity crisis due to definitional issues ... It's in danger in many places ... the Minister of Health in the US got up and started talking about throwing people in jail as a harm reduction measure, and that would be very much against what the notion of harm reduction is about ... (Hathaway, 2001, p. 127).

Literally, people who use drugs cannot cause harm to society (or be harmed) if they are not actually in that society. This kind of pseudo harm reduction notion leads to the criminalisation and detention of people who use drugs in prison and compulsory treatment centres, as explored further below in the Vietnamese context. In addition, considering the detention of people who use drugs in jails or prisons as a measure to reduce harm is linked to a 'full recovery' approach, which requires people who use drugs to abstain from drugs when joining 
treatment. This is arguably a coercive approach that could undermine harm reduction. Recovery to become 'drug-free' has generally been adopted by the abstinence movement to confront drug use (Monaghan \& Bewley-Taylor, 2013), but the insistence on full recovery can also be seen as a new mechanism for controlling people who use drugs (Roy \& Buchanan, 2015; Seddon, Ralphs, \& Williams, 2008). Although abstinence is an approach that does help some people who use drugs, imposed or forced abstinence, like sending people to compulsory treatment centres, or imposing abstinence through drug courts, is problematic. In other words, abstinence within a prohibitionist punitive framework is problematic, not people who use drugs aiming for abstinence per se.

\section{'Authentic' Harm Reduction}

'Authentic' harm reduction seeks to mitigate the harms caused by drug use within a framework that promotes human rights and humanistic values towards people who use illicit substances, rather than seeking to prevent drug use (Hunt et al., 2003; Wodak, 1998). Importantly, it aims to reduce harms caused by prohibitionist drug policies. Based on the definition from Harm Reduction International (Harm Reduction International, 2016), harm reduction is defined for this study as follows:

'Authentic harm reduction' refers to compassionate and pragmatic policies, programmes and practices that aim primarily to reduce the adverse health, social and economic consequences of the use of illegal drugs without necessarily reducing drug consumption. Its cornerstones are public health, human rights and social justice.

Authentic harm reduction is a pragmatic and flexible approach that places people who use drugs at the centre of addressing their drug problems, instead of locking them up for drug use, or insisting that they have to stop using drugs altogether. The next section further discusses the key principles of authentic harm reduction in response to drug use related problems and illustrates the differences between harm reduction and abstinence-based approaches. 


\section{Key Principles of Harm Reduction}

Although harm reduction has developed over the last few decades, it still remains controversial in philosophy and practice (Collins et al., 2012). The controversy around harm reduction philosophies, their implementation and application have raised concern about how harm reduction should be understood and implemented in a way that is more effective and can work with current prohibitionist drug policies (Collins et al., 2012; Roe, 2005). Generally, harm reduction is argued to be opposed to prohibition and abstinence (See Appendix One for detailed comparisons). Arguably, the key principles of harm reduction are based on three main determinants: (i) conceptualisation of drug use, (ii) responses to drug use, and (iii) harm reduction and relationships of power. Harm reduction principles include accepting that all drugs (both legal and illegal) are an essential part of human life and that abstinence should not be seen as the ultimate goal of drug treatment and drug policy. Also, harm reduction highlights the importance of people who use drugs' roles in addressing their addiction. Importantly, harm reduction focuses on mitigating the multiplicity of harms towards users like stigma, discrimination and human rights violations as well as promoting drug policy reform. Understanding harm reduction principles is necessary to properly implement corresponding interventions in practice. Firstly, this section critically explores how harm reductionists conceptualise drug use. From here, it illustrates and analyses harm reduction responses to drug use, and it then shows the relationships of power which guide harm reductionists on how people who use drugs should be treated.

\section{The Conceptualisation of Drug Use}

As noted previously, the current category of 'drugs' is a socially constructed one. All illegal drugs are perceived as being dangerous because those substances are listed under the UN Conventions ${ }^{24}$ on illicit drugs. Other legal drugs like alcohol or tobacco are even more dangerous and harmful than some illegal drugs, like cannabis or ecstasy, but this fact is generally ignored within governmental agendas (Nutt, 2012). Further, given that the UN Conventions are based on a mid-20 $0^{\text {th }}$-century sociocultural construct (with no coherent

\footnotetext{
${ }^{24}$ The Single Convention on Narcotic Drugs 1961, the Convention on Psychotropic Substances 1971 and the Convention against the Illicit Traffic in Narcotic Drugs and Psychotropic Substances 1988.
} 
pharmacological rationale), many governments fail to acknowledge that alcohol, tobacco or caffeine should also be classified as drugs (Taylor et al., 2016). In reality, alcohol and tobacco are the most commonly used psychoactive drugs and the reasons for using them mirror the use of other drugs, including illegal substances (Goldberg, 1999). Also, as the Global Commission on Drug Policy (2017, p. 10) affirms, 'there are risks involved in drug use, regardless of whether the substances involved are legal or illegal'. Whether or not drug use is risky and harmful, it has been recorded throughout human history (as discussed above). Therefore, harm reductionists acknowledge that drug use is a reality of human nature and that it is unrealistic to stop people from using drugs (Hofschulte, 2012; McNeece, 2003; Stevens et al., 2010). It is the case that, whether or not people accept drugs, drugs still exist and continue to be an important part of human's lives (Gossop, 2012). Only a minority of illegal people who use drugs develop serious drug problems, and the vast majority of people who use drugs are recreational users who consume drugs in a controlled manner (UNODC, 2015, 2016b).

In contrast to the abstentionists, harm reductionists identify that the reasons for using illicit drugs are not different to the motivations for legal drugs: as a pleasurable habit, to be social, to unwind, and to enjoy the effects of drugs (Buchanan, 2009; Coomber et al., 2013; Nutt, 2012). The effects and benefits of drugs to users are evident, either good or bad, so drug use is unavoidable in our societies. Duff $(2015$, p. 1$)$ argues that drug use can be regarded as 'a practice of care'. Specifically, he explains that drugs, either legal or illegal, help users to moderate the symptoms of mental stress and help them to live a 'better' life. Similarly, Race (2008) highlights that taking drugs is seen as a mechanism to overcome material and social disadvantage. Therefore, drug use is viewed as a coping mechanism and an inevitable part of everyday life (Duff, 2015). For instance, drugs are seen as a form of pleasure and purpose, like chatting over beer, opening a bottle to celebrate or to relax, or drinking Red Bull to concentrate. Essentially, people who use drugs should not be judged, then, on the grounds of drug use behaviour. This attitude is important because authentic harm reduction is a nonjudgemental approach to reducing drug-related harms.

The use of illicit drugs is not the central distinguishing characteristic that defines a 'drug user' (Stafford, 2007). Stafford (2007) argues that, except for medical purposes, it is apparent that the use of drugs for a range of purposes is an accepted and normal part of most - if not all - 
human societies. Similarly, Taylor et al. (2016) highlight that it is undeniable that legal drugs like alcohol, tobacco or even coffee are clearly psychotropic and stimulant substances, like illicit drugs. Harm reductionists, therefore, claim that no distinctions should be made between legal drugs, pharmaceuticals and illicit drugs because all drugs have both advantages and disadvantages, and all drugs are potentially dangerous and pose risks, even though some are less harmful than others (Goldberg, 1999; Hunt et al., 2003). Accordingly, harm reductionists view drug consuming behaviours as normal, and that drug use behaviour should be respected (Goldberg, 1999). In addition, they accept that consuming and possessing a certain amount of drugs for personal use should not be subject to prosecution or punishment; in other words they promote the decriminalisation of drug consumption and drug possession, and view that some less harmful drugs (like cannabis) might be legalised (Goldberg, 1999; Harris \& McElrath, 2012). While abstentionists treat people who use drugs as a special category (because, as noted in Chapter Two, people who use drugs are seen as a source of social problem/crime), harm reductionists respect and promote people who use drugs' rights (Stevens et al., 2010; Stimson, 2007; Wodak, 1998). Tammi and Hurme (2007) further highlight that people who use drugs have a right to life, security and full citizenship and to be free from discrimination. Considering drug use from a neutral perspective, not a judgemental or moral one, is vital when it comes to responding to drug use from the standpoint of harm reduction.

\section{Responses to Drug Use}

Marlatt, Marry, and Witkiewitz (2012) argue that any programme and intervention that does not require abstinence and seeks to reduce harm could be considered harm reduction. Also, as Buchanan (2015b), Levy (2014), and Ritter and Cameron (2006) state, a harm reduction approach is more pragmatic because the use of law enforcement to control drug use is often counterproductive. For example, in the USA, the violent crime, gang warfare, prison overcrowding, and police corruption associated with prohibition strategies have reached such a level that policy-makers, practitioners, and members of the public alike are attracted to harm reduction because of the counterproductive effects of prohibition together with the spread of the HIV epidemic (Riley \& O'Hare, 2000). Similarly, at some points, Vietnam adopted some medical interventions in the name of harm reduction when the HIV infection became a serious problem and the country failed to prevent drug use and addiction (Windle, 2015). Yet 
it is important to reaffirm that Vietnamese harm reduction has been practised under punitive regimes, such as 06 centres or police crackdowns.

Harm reduction is also based on respect for human behaviour and non-judgemental attitudes when it comes to drug use because drug use is part of a universal history of humanity. Therefore, people who use drugs should not be judged because they use drugs. Denning (2001) argues that within the harm reduction framework, acknowledging drug use as a highrisk behaviour is a product of a given time and culture, and associated values, norms and beliefs. A culture of fear of a particular social group, for example, is seen as a driver for punitive policy and regulation related to drug use (Hutton, 2017). As Buchanan (2016a) argues, 'people can be harmed by drugs, but most harm is caused by prohibitive and intolerant drug policies ... drugs are dangerous but criminalisation is worse' (p. 1). Therefore, criminalising people because of drug use behaviour can be seen as going against legitimate human rights. As Shelly $(2016$, p. 1$)$ asserts:

If you're not talking decriminalization, you cannot call yourself a harm reductionist. Decriminalization should be top of the agenda not because "the current policies haven't worked," but because drug use should never have been criminalized in the first place. Decriminalization is the easiest, most immediate change that can be made to the policy landscape and it would significantly reduce the harms associated with drug use. Our aim is not to rid the world of drugs, nor to stop people from using drugs-both are unachievable and, on closer inspection, undesirable. Our aim is to mitigate the significant harms that drug policies have caused.

Harm reduction is also heavily based on a low-threshold approach in which people who use drugs are encouraged to participate in harm reduction interventions with minimal requirements imposed upon them (Blalock-Wiker, 2014; Denning \& Little, 2011; Hilton et al., 2001). This means that this is not a coercive approach. In Vietnam, for example, most people with active drug use problems do not seek treatment on a voluntary basis because of stigma and discrimination and the risk of long-term detention in compulsory treatment centres (Vuong et al., 2016). In Vietnam, those who end up in treatment are often forced to by courts or other legal requirements (Human Rights Watch, 2012; Khuat et al., 2012). Forcibly detaining people who use drugs for treatment purposes is contrary to authentic harm 
reduction principles, which are based on a low threshold approach. In short, from a harm reductionists' perspective, responses to drug use should be rooted in the ideas of humanism and a non-judgemental approach, and focus on reducing the harms associated with drug use rather than punishing and controlling people who use drugs.

\section{Harm Reduction and Relationships of Power}

Unlike an abstinence-based approach, which is often accompanied with punishment or prohibition, and tends to enhance the people who use drugs' shame, guilt and accountability, harm reduction is a humanistic and non-judgemental approach based on people who use drugs' opinions about how they want to address their drug-use problems (Marlatt \& Witkiewitz, 2010). Therefore, the role of service providers is to help users to reduce the harms caused by drug use without judgemental attitudes (Marlatt \& Witkiewitz, 2010). In situations where people who use drugs become addicted and continue to use drugs and relapse, harm reductionists argue that relapse is acceptable in drug treatment and it should be seen as a natural and expected occurrence (MacMaster, 2004; Marlatt, 2000). If people who use drugs struggling with addiction continue to use drugs after treatment, this should not be viewed as an individual's failure or lack of ability but, instead, should be seen as a learning experience (MacMaster, 2004). Also, Tatarsky (2007, p. 249) asserts that:

The essence of harm reduction is the recognition that treatment must start from the client's needs and personal goals and that all change that reduces the harms associated with substance use can be regarded as valuable.

Therefore, people who use drugs should be advised that they do have the ability and right to change their own addictive behaviour and can even continue their drug use as long as they do not harm others. In reality, as Denning and Little (2011) explain, many people worry that if people who use drugs are given the right to take drugs and decide their treatment goals, this will lead to addiction and problems. However, harm reductionists assert that only a minority of users will struggle with addiction; most users will not, as detailed above (Denning, 2001; Denning \& Little, 2011).

As noted previously, respecting individual dignity and self-determination without judgemental attitudes are vital values of authentic harm reduction (Richard \& Riley, 2012; 
Riley et al., 1999), so harm reductionists propose that attention should be placed on giving people who use drugs the opportunities to make well-informed choices (Goldberg, 1999, 2004). As a consequence, people who use drugs are regarded as the best source for dealing with their problems and they should be empowered to discuss with service providers which intervention is best suited to their needs (Fernandez, 2012). Authentic harm reduction is most often individualised to the special needs and wants of the individuals and community; it is not a one-size-fits-all approach (Marlatt \& Witkiewitz, 2010). This is contrasted with the abstinence approach in which the clinician plays an 'expert' role and instructs their clients on what to do (Miller, 2002). Promoting people who use drugs' rights facilitates their motivation to make behaviour changes, which is important to overcome their drug use problems (Miller \& Rollnick, 2012). In other words, allowing people who use drugs to make decisions on addressing their problems is consistent with the arguments that drug use and addiction are complex and rely on social, economic and environmental factors (the setting) rather than the drug and the set.

\section{Harm Reduction, Human Rights and Drug Policy Reform}

As detailed above, harm reduction has largely been considered from a public health approach (Erickson, Riley, Cheung, \& O'Hare, 1997; Harm Reduction International, 2016; Marlatt \& Witkiewitz, 2010). Nevertheless, in recent years, harm reduction has been viewed as a response to the deficiencies of prohibition and abstinence, as researchers have argued that prohibitionist policies threaten the freedom of people who use drugs, damage their health and constitute them as marginal and stigmatised groups (Elliott et al., 2005). For example, Hathaway (2001) and Ezard (2001) assert that human rights are not only at the core of harm reduction, but they offer a necessary moral grounding for arguments for social change and policy reform. In particular, they state that prohibition is not only irrational, but is also immoral because it violates the autonomy of the individual. Likewise, Buchanan (2015b) argues that 'the individual must have sovereign right over their own body, to consume what they wish, without fear, threat or punishment from the state' (p. 70). Shelly $(2016$, p. 1) further explains why harm reduction needs to consider people who use drugs' rights, and argues: 
I regularly use examples that show how HIV can spread from people who inject drugs into the wider community-drawing the conclusion that in order to protect the wider community, we need to protect people who use drugs. Why the need to justify lifesaving interventions? People who inject drugs deserve protection, periodregardless of any impact on the "wider population." And, by the way, people who use drugs and "the wider community" are inseparable; they are one and the same.

However, Keane (2003) deems that adopting a discourse of human rights will not provide an end to the debates about harm reduction and its principles. She explains there is inevitably a fundamental tension between a demand for care and protection by the state for people who use drugs and a demand for care and protection for the wider community. Likewise, Johnson (1993) argues that 'human rights may limit harm reduction's usefulness in the field of drug use. He notes that the rights of people who use drugs will inevitably go against other groups, such as the rights of children (to have drug-free parents), the rights of others (to be protected from the harms of drug use) or the rights of taxpayers (not to pay for medical costs of people who use drugs)' (Johnson, 1993, p. 7). Yet as Buchanan (2015b) challenges, generally we are all people who use drugs (at least using caffeine, alcohol or tobacco), so it is not really rational to argue that ensuring the rights of the people who use drugs will come up against the rights of others. Denying the rights of people who use drugs is to justify prohibition and to protect legal drugs. For example, Buchanan (2015c, p. 1) argues:

It (prohibition) protects the market share and status of the privileged, promoted and culturally embedded legal drugs - alcohol, tobacco, sugar and pharmaceuticals ... It provides a much needed distraction from the serious problems caused by the more harmful, addictive and culturally embedded legal drugs.

In practice, promoting the legitimate rights of people who use drugs is challenging. For instance, as Keane (2003) explains, embracing human rights is likely to increase the political obstacles faced when conducting harm reduction because current drug laws are mainly to protect society rather than people who use drugs. From this standpoint, promoting the rights of illicit people who use drugs means that harmful behaviours are accepted or undesirable conduct is reinforced (Hathaway, 2001). This argument is generally the case in South East Asia 
and Vietnam where drug use is regarded as a 'social evil' (Windle, 2015). Riley and O'Hare (2000), for example, also raise concerns that:

Even though I would never define harm reduction without it including human rights changes, in most countries, trying to sell anything on a human rights platform is useless. Especially, that's true in places like Malaysia. Well you know, forget it; you have to find some other way in there (Cited in Hathaway, 2001, p. 133).

Similarly, Edington and Bayer (2013) highlight this issue with regards to the implementation of harm reduction in Vietnam where 'human rights' are a sensitive matter. They give an example of human rights' issues as working with Vietnamese government officials:

I think that if we had been coming in and we had been talking about ... access to health and human rights, some of these more holistic structural approaches to the issue of drug use, I think the door would have been slammed upon us (Edington \& Bayer, 2013, p. 78).

However, many advocates like Marlatt et al. (2012) or Riley et al. (1999) argue that drug policy will eventually evolve in the search for more humane longer term solutions. Similarly, Hathaway (2001) and Ezard (2001) argue that a focus on human rights is important to harm reduction because this highlights the responsibility of the state to the vulnerability of individuals and their risk of drug-related harm. For Ezard, the state should ensure human rights of people who use drugs because the failure of ensuring human rights will increase the likelihood of stigma and discrimination towards people who use drugs, which will arguably cause harms not only to individuals, but the wider community. Similarly, Levy (2014) and the International Harm Reduction Association (2016) both argue that when people who use drugs are at risk from and suffer human rights abuses, harm reduction services can never properly function, and drug-related harms, like HIV infection and other blood-borne diseases, will be exacerbated. In addition, many researchers like Buchanan (2015b), Fedotov (2010) or Elliott et al. (2005) highlight that, if people who use drugs' rights, such as to health, education or employment, are jeopardised, this will further isolate users from normative society, and reduce their opportunities for education, outreach or employment. All of this would exacerbate harm to people who use drugs, and others in the wider community - as in Russia, where needle exchanges are outlawed, the HIV rate is rising compared to other countries 
where it has been steadily declining (Tkatchenko-Schmidt, Renton, Gevorgyan, Davydenko, \& Atun, 2008). Therefore, a human rights vision and practice are required, even though the human rights discourse is still minimised.

Amid these debates, harm reduction has begun to influence national drug policies, such as promoting the decriminalisation, legalisation and regulation of drugs (Erickson, 1999; Lishchynski, 2004). For example, in 2001, Portugal decriminalised all personal possession of drugs, while the Netherlands has made cannabis widely available in coffee shops across Amsterdam since the 1990s. Another striking example is Switzerland where, since 1994, the government has allowed doctors to prescribe free clean pharmaceutical heroin ampoules to those addicted, and to provide medically supervised rooms for injection (Buchanan, 2015b). In 1999, Vietnam adopted a model of 'decriminalisation'25 of drug use, and in 2006 officials adopted so-called harm reduction with the launch of the 2006 HIV Prevention Law. As discussed at length below, this has been stymied by the continued emphasis on abstinence, and the punishment and compulsory treatment of people who use drugs. This has ensured harm reduction remains deeply limited in practice (Windle, 2015). As Taylor et al. (2016) argue, abstinence/prohibition remains the primary source of harm for illicit and injectable people who use drugs and creates plenty of unintended consequences. Meanwhile, authentic harm reduction strategies can produce a wide range of positive outcomes.

\section{The Positive Outcomes of Harm Reduction}

By contrast, the effectiveness of harm reduction interventions in reducing the harms associated with drug use is evident. For example, needle and syringe exchange programmes (NSPs) are typical medical harm reduction interventions and they help to reduce the risks of infectious diseases such as HIV or Hepatitis C (Thomson, 2013), but also help people who use drugs access harm reduction support by building relationships and understanding of treatment services as well as providing them with necessary information about safer injecting drug use (Blalock-Wiker, 2014). NSPs have succeeded in preventing the expansion of HIV,

\footnotetext{
${ }^{25}$ According to the 1999 Vietnamese Criminal Law, drug possession for personal use is decriminalised and drug use is seen as a public health matter, not a criminal one. This is an important milestone regarding the promotion of harm reduction in Vietnam. This will be further discussed in Chapter Five.
} 
Hepatitis C and other blood-borne diseases (Blalock-Wiker, 2014; Doherty et al., 2000; Gibson et al., 2002; Huo, Bailey, Hershow, \& Ouellet, 2005; Satcher, 2000). Another example is opioid substitution treatment. This therapy provides people who use drugs with a replacement drug or a prescribed medicine (like methadone) that is medically tested regarding its effects (Kermode, Crofts, Kumar, \& Dorabjee, 2011). The effectiveness of the substitution intervention is high in terms of reducing illicit opioid use, morbidity and death from overdose, and decreases people who use drugs' involvement in crime as well as HIV risk behaviours (Kermode et al., 2011; White, Dore, Lloyd, Rawlinson, \& Maher, 2014; Wodak, 2011).

Further, many countries like the USA, Canada, Australia or the Netherlands implement overdose prevention interventions by providing naloxone ${ }^{26}$ and 'drug checking' ${ }^{27}$ to users, their family and relevant police officers (International Harm Reduction Association, 2016). These interventions not only reduce the likelihood of overdose, but improve the likelihood of a positive medical response to an overdose (Ritter et al., 2011). Naloxone, for instance, has no psychoactive properties, does not increase drug use demand, and has no abuse potential (Mueller, Calcaterra, Glanz, \& Binswanger, 2015; Van Dorp, Yassen, \& Dahan, 2007; Wheeler, Davidson, Jones, \& Irwin, 2012). The aforementioned successful examples have confirmed the effectiveness of pragmatic and low-threshold approaches of harm reduction in addressing drug use related problems in practice.

Drug consumption rooms are also a successful model of harm reduction. Eighty-eight drug consumption rooms (DCRs) have been implemented in ten countries, not including Vietnam (International Harm Reduction Association, 2016). These facilities allow people who use drugs to access clean equipment and to legally inject or inhale drugs in a user-friendly medically supervised space. This intervention has been very effective in reducing overdoses, and increasing use of health and social services, as well as minimising risks associated with drug

\footnotetext{
${ }^{26}$ Drug overdose medicine that reverses the effects of opiate overdose.

${ }^{27}$ Drug checking aims to send targeted preventive messages to recreational people who use drugs. This approach, which is more individualised than mass media campaigns, provides an incentive for people who use drugs to participate in a dialogue about prevention and harm reduction. People who use drugs will be advised of the test results, so that they know what particular drugs they are consuming and how to prevent harmful effects (Brunt, 2017). Free drug checking is a harm reduction service that tests the safety of recreational substances. It is recently available in New Zealand on a limited basis.

See more: https://www.drugfoundation.org.nz/info/being-safer/party-drug-checking/
} 
use (Wood, Tyndall, Montaner, \& Kerr, 2006). For example, between May 2001 and April 2010, the Medically Supervised Injecting Centre in Sydney (Australia) was able to intervene and assist with 4,400 overdoses and not a single case resulted in a fatality (Schatz \& Nougier, 2012). Another striking example of DCRs is InSite, which was opened in 2003 in Vancouver with the agreement of the Canadian Government, to become the first legal supervised injection site in North America. The mission of InSite is to create a safe environment where people who use drugs are provided with sterile needles, and physical and mental health assistance from medical staff. Importantly, it contributes to alleviating adverse health, social and economic consequences caused by drug use without requiring abstinence. InSite has a team of nurses, peer support workers, counsellors, and mental health workers who are available to provide clients with health care but also, more importantly, assistance with housing, addiction treatment, and other supportive services.

While InSite is a good model of harm reduction, the Portland Hotel Services Society (PHS) can be seen as a role model of comprehensive harm reduction strategies. In 1991, the PHS was founded in Vancouver, Canada, with the express purpose of providing appropriate and affordable accommodation for 'the most hard to house', namely people who use drugs, HIV infected people, and other people living with mental-health illness or with trauma for many years (Gurstein \& Small, 2005). The PHS provides those people with a 'home', where they live in dignity, and with a sense of comfort and security, more than just a shelter. Initially, the PHS offered services to help people prevent drug-related overdoses and mitigate HIV infection among people who use drugs. It then extended its scope, by organising events, classes and programmes to educate users on how to reduce harms associated with drug use, as well as by providing in-house needle exchange and low threshold methadone programmes for people who use drugs. Notably, the PHS not only focuses on health issues, but rather it also addresses social inclusion, promotes human rights, restores social capital and improves quality of life for people who use drugs (Small, Palepu, \& Tyndall, 2006). The PHS gives a drug user an opportunity to live as a normal person, not a criminal, a diseased person or an outsider, and it also helps some of the most marginalised and vulnerable to reintegrate into the community. A good example of assisting people who use drugs to 're-enter' society is opening a bank account for them (Burger, 2004), which enables people who use drugs to cash cheques, pay bills, or get money orders. This helps them operate more easily in increasingly 
'cashless' societies, thereby enhancing self-respect and social inclusion. The PHS is cost effective and has a positive impact on saving lives, decreasing crime and improving social order (Bayoumi \& Zaric, 2008; Winkelmayer, Weinstein, Mittleman, Glynn, \& Pliskin, 2002). Again, the Canadian example demonstrates that a humanistic and non-judgemental approach of authentic harm reduction has created significant changes in the lives of many people who use drugs.

Highlighting these positive outcomes of harm reduction, Ashton (2008) and the International Harm Reduction Association (2016) argue that harm reduction should be integrated, as a goal, in government drug policies. For example, governments should regulate the drugs that people consume and educate them about the possible side effects of those drugs. Also, large international organisations like UNAIDS and WHO have recommended the worldwide expansion of harm reduction that promotes public health law reform efforts (Collins et al., 2012). Harm reduction is not only popular in Western Europe and North America, but it is integrated into the drug policies of Asian, Africa, South American and Eastern European countries as a response to drug use (International Harm Reduction Association, 2016). Nonetheless, given the global dominance of abstinence and prohibitionist based drug policy, it is vital to comprehend the key critiques and challenges of harm reduction that have been encountered in practice in order to further promote authentic harm reduction.

\section{Critiques and Challenges of Harm Reduction}

Almost as soon as the progressive, pragmatic and effective concept of harm reduction was widely introduced in the 1980s, there was also criticism of this approach (Keane, 2003). There are some main criticisms, including that (i) harm reduction fosters drug use, (ii) it keeps people who use drugs 'stuck', (iii) it opposes addiction treatment targets, (iv) it promotes 'middleclass paradigms', (v) it does not work for every drug user, (vi) legal frameworks hamper harm reduction, and (vii) harm reduction faces chronic under-funding. It is important to note that these criticisms largely reflect misapprehensions about harm reduction or the social or political context in which it is implemented, rather than intrinsic problems of harm reduction.

The first criticism is that harm reduction promotes drug use. The global responses to drug use are rooted in a regime of drug prohibition and the belief that abstinence is the only way to help people who use drugs (Kyser, 2010). For instance, some perceive that if the government 
assists those already using drugs to remain healthier, avoid problems and stay alive, people who do not use drugs will regard drugs as safe and may try to use drugs (Hunt et al., 2003). As a result, harm reduction is thought to 'send out the wrong signal' or 'send a mixed message' to the community (Hunt et al., 2003; Kyser, 2010). To those who support a 'drugfree society', a harm reduction approach also means that the government is complicit in ongoing drug use (Kleiman, Caulkins, \& Hawken, 2011). Abstentionists, for instance, argue that providing free needles and syringes to intravenous people who use drugs increases the rate of progression to injecting use (Hathaway, 2001). However, the evidence on the implementation of needle exchanges and other harm reduction programmes shows that they do not trigger an increase in drug use (O'Hare et al., 1992; Riley \& O'Hare, 2000; Strathdee et al., 1997). Further, Hunt et al. (2003) argue that drug use is itself a dynamic phenomenon that may independently increase and decline over time, so blaming harm reduction for increasing or decreasing drug use is difficult for both its critics and advocates. Hunt and his colleagues (2003) emphasise that all drugs, either legal or illegal, are potentially harmful, but the harms can, to some extent, be minimised and constrained by harm reduction. Also, many researchers like Erickson et al. (1997), Kyser (2010) or Riley et al. (1999) contend that considering harm reduction as a means of promoting drug use seems to underestimate the complexity of drug use behaviours and the factors that shape people's decision to take drugs.

Another reason for criticising harm reduction is that it keeps people who use drugs 'stuck'. As McKeganey (2011) argues, harm reduction may allow people who use drugs to continue to use drugs and perhaps to still use some types of drugs for a long time, and this makes many people worry that becoming drug-free seems to be out of reach for people who use drugs or that they have no incentive. Yet the idea that people would be again dependent on drugs, or are stuck, reflects a classic individual pathology from abstentionists (Marlatt et al., 2012). Those who see the problem as substitute prescribing (harm reduction) fail to take into account other structural factors; in fact, these people are stuck not because of prescribing but because:

When considering treatment and rehabilitation it must be recognized that many problem people who use drugs have had such limited options in life, that they lack personal resources (confidence, social skills and life skills) and have limited positive life experiences to lean upon or return to. This client group need social integration not 
social reintegration, they need habilitation not re-habilitation - it seems that many have never really been able to get started in life in the first place. This makes living without drugs a very tough option indeed (Buchanan, 2004a, p. 393).

In addition, providing people who use drugs with prescribed injectable heroin is another example of the argument that harm reduction keeps people who use drugs 'stuck'. This harm reduction approach is arguably opposed to addiction treatment targets, which impose drug intake reduction (Andersen \& Jarvinen, 2007; Hathaway, 2001). Yet harm reductionists support prescribed injectable heroin due to its positive outcomes, and because it meets the needs of problematic drug users who need pharmaceutical grade heroin for overdose prevention or to overcome their addiction (Buchanan, 2016b). Providing problematic or heavily addicted people who use drugs with pharmaceutical grade heroin reflects a progressive approach of harm reduction (delivering care wherever needed). Moreover, (Buchanan, 2016b) argues that imposing a reduction target does not reduce harm, it increases it - because the person would end up using dangerous adulterated street drugs. Further, a wide range of studies show that heroin-assisted treatment programmes are highly effective, as they contribute to reducing illicit opioid use, morbidity and death from overdose, and decrease people who use drugs' involvement in crime as well as HIV risk behaviours (Kermode et al., 2011; White, Dore, Lloyd, Rawlinson, \& Maher, 2014; Wodak, 2011).

Another challenge to scaling up harm reduction is that opioid substitution treatment (OST) may largely be effective with patients who abuse opioids, but it may be less successful for candidates who do not have an opioid problem, or those who are non-opioid people who use drugs (using other drugs) or stimulant people who use drugs (Grella, Anglin, Wugalter, Rawson, \& Hasson, 1994). For instance, methadone is not the right answer for synthetic drugs like methamphetamine or synthetic cannabinoids (Harm Reduction International, 2016). However, the effectiveness of methadone maintenance in reducing illicit opioid use, morbidity and death from overdose, and decreasing people who use drugs' involvement in crime as well as HIV risk behaviours, is evident in many countries (Ducan, Nicholson, White, \& Ellis-Griffith, 2014; Kermode et al., 2011; Wodak, 2011), Vietnam included (Hoang et al., 2015; Reid \& Higgs, 2011; Tran, Ohinmaa, Duong, Do, Nguyen, Nguyen, et al., 2012; VAAC, 2011). Therefore, 80 countries and territories, including Vietnam, implemented OST in 2016 (International Harm Reduction Association, 2016). 
Furthermore, harm reduction may be criticised for imposing 'middle-class values' on drug consumption (Miller, 2001, p. 175). That is, harm reduction assumes 'harm' is occurring and, in doing so, it cites some users of some drugs as 'risky' and 'dangerous'. As Petersen and Lupton (1996, p. 192) argue, a middle-class paradigm 'connotes a group considered both at risk of contracting HIV and at risk of transferring it'. As a result, people who use drugs are expected to be responsible for staying healthy, not only for them, but for others. Yet these discourses are dangerous for people who use drugs because they are viewed as the greatest risk group to the wider community, leading to further stigma and discrimination against them (Miller, 2001). In other words, as Kellehear (1993) and Keane (2003) argue, middle-class values recall the moral discourse about some drugs and people who use drugs and reproduce categories, such as 'abnormal' and 'pathological'. Accordingly, people who use drugs continue to be kept under surveillance, while social issues, such as poverty and inequality, are ignored. Yet as noted previously, current harm reduction movements focus on reducing harm caused by drug use for all people who use drugs without judgmental attitudes. Authentic harm reduction also seeks to reduce the harms associated with drug policy and laws.

Legal challenges like 'drug paraphernalia laws' 28 and other drug control laws can also stand in the way of harm reduction. As detailed previously, the current global responses to illicit drugs rest upon three international conventions: the 1961 Single Convention on Narcotic Drugs, the 1971 Convention on Psychotropic Substances, and the 1988 Convention against Illegal Traffic in Narcotic Drugs and Psychotropic Substances (Elliott et al., 2005). As a consequence, drugrelated activities such as the production, distribution, trading or possession of illicit drugs tend to be criminalised. In other words, as Hilton et al. (2001) argue, drug laws have prioritised 'zero tolerance'. In this context, providing prescribed injectable heroin or opening drug injection rooms can be seen as distributing drugs to users, which is opposed to the drug

\footnotetext{
${ }^{28}$ In drug laws, this entails making unlawful possession of any equipment, product, or material of any kind that is primarily intended or designed for use in manufacturing, compounding, converting, concealing, producing, processing, preparing, injecting, ingesting, inhaling, or otherwise introducing into the human body a controlled substance. See more https://www.law.cornell.edu/uscode/text/21/863.

This kind of law hampers harm reduction in practice. For example, it prevents people who use drugs from carrying needles and syringes, or from accessing these items from harm-reduction services. In Vietnam, for instance, many people who use drugs are scared of carrying sterile needles and syringes because of the risk of police harassment and even arrest.
} 
control law. In practice, many users can be arrested for either taking contaminated used needles (with traces of drugs in them) to needle exchanges or for possessing sterile needles because they are classed under the law as drug paraphernalia. Therefore, in Australia, for instance, the consumption room in Sydney has negotiated tolerance zones around them with local police so that people who use drugs will not be arrested (Elliott et al., 2005).

Similarly, Hathaway (2001) indicates that harm reduction is generally vulnerable to debasement by proponents of drug control laws. This challenge is evident in Vietnam. There, although drug use has been decriminalised since 1999, the country still pursues a zerotolerance approach (Vuong et al., 2012). For example, drug possession is decriminalised, but people who use drugs still can be detained in 06 centres for an unspecified period in the name of an administrative violation (further discussed in Chapter Five). In reality, introducing needle exchanges, methadone, prescribed injecting heroin or drug injection rooms is viewed as contrary to Vietnam's drug policy, which targets building a drug-free society (Edington \& Bayer, 2013). However, the success of harm reduction interventions in the UK, Portugal, Netherlands, Canada and Switzerland (as noted previously) has demonstrated that harm reduction approaches can work well despite criminal justice systems and a prohibition approach to drugs (Buchanan, 2015b). In fact, harm reduction has been effective in not only addressing medical issues associated with drug use, but also in helping people who use drugs to improve their health, social relationships and facilitate their (re)-integration into society.

In addition to dealing with legal challenges, harm reduction also more often than not faces chronic underfunding (Harm Reduction International, 2016). International donor funding allocated to harm reduction is in decline, especially in middle-income countries (MICs) where harm reduction is most needed (Harm Reduction International, 2016). For example, in Brazil, harm reduction workers often experience frequent discontinuation of payments while still working in the field, mostly because the government is not able to secure grants on a continuous basis (Domanico \& Malta, 2012). Meanwhile, in South East Asia, harm reduction is often in conflict with mainstream government approaches to drug use; thus, it often encounters underfunding (Thomson, 2013). In many countries, in order to build a drug-free society, supply control is the focus, while only tiny proportions of budget are spent on harm reduction measures compared to law enforcement responses. For example, in Australia, most funding was allocated to law enforcement (66\%) and treatment (21\%) and other programmes 
(9\%), while funding for harm reduction was only $2 \%$ (Ritter et al., 2011). Similarly, the funding that the Vietnamese government allocates to harm reduction is always much lower than abstinence-based approaches like demand and supply reduction (Vuong et al., 2017). When Vietnam moved towards middle-income status in 2010, international funding for harm reduction sharply reduced because the international funding is regulated to prioritise poor countries (Hammett et al., 2008; Reid \& Higgs, 2011; Vuong et al., 2012).

Globally, it was estimated that US\$100 billion was spent on drug control in 2012, while only around US\$8.9 billion was allocated for the response to HIV, harm reduction included (Cook, Bridge, McLean, Phelan, \& Barrett, 2014). In 2015, funding for the HIV response and harm reduction services dropped to US\$8.2 billion (International Harm Reduction Association, 2016). Many advocates propose redirecting $10 \%$ of funding for drug law enforcement to harm reduction, but this has not occurred (Harm Reduction International, 2016). According to HRI and the Burnet Institute, such a redirection has the potential to virtually end AIDS among people who inject drugs. Yet while governments, politicians and media prioritise abstinence, the scientific evidence and efficiency of harm reduction will continue to be underestimated (Harm Reduction International, 2016). The underfunding arguably is a short-term problem. The underestimation and misleading perceptions of harm reduction should be seen as the long-term hurdles for effective harm reduction (Rhodes \& Hedrich, 2010). The chapter on harm reduction in Vietnam (Chapter Five) and the findings chapters will further illustrate why misperceptions remain a major challenge to harm reduction.

\section{Conclusion}

'Authentic' harm reduction is a relatively underdeveloped social policy with respect to drugs, because it looks at drug use from various psychological, sociological and environmental perspectives (Riley \& O'Hare, 2000). Authentic harm reduction engages with the diversity of human experiences and behaviours in relation to drug use. The key principles of authentic harm reduction reflect how and why drug use should be perceived and how people who use drugs should be treated in order to address their struggles in negotiating prohibition, their use of illegal drugs, as well as the community and social problems associated with illicit drug use. Harm reductionists try to influence the public, government workers and international organisations to develop human rights based laws, policies and practices to live with drugs 
(legal and illegal) and to minimise their harm. Harm reductionists respect personal life choices without judgemental attitudes, regardless of whether people are involved with drugs or not. Importantly, harm reduction is a socially inclusive strategy, aimed at integrating dependent people who use drugs into society rather than socially isolating them (Webster, 2007). Therefore, it contributes to reducing the international and national harm caused by prohibition and drug abstinence-based approaches.

Throughout its history, most countries have conducted harm reduction from narrow public health perspectives (addressing HIV infection, other blood-borne diseases and addiction), but recently public health is no longer the sole focus of authentic harm reduction (Harris \& McElrath, 2012). Over the course of the last decade, harm reduction has expanded its scope to address the multiplicity of harms associated with drug use at the individual, community and societal level (Marlatt et al., 2012). Noticeably, many harm reductionists have increasingly used 'human rights' as the discursive framework for harm reduction (Stimson, 2007). Some harm reductionists even go further than public health and human rights, and promote drug law and policy reform that emphasise decriminalisation, regulation or legalisation (Elliott et al., 2005; Ezard, 2001).

Although there is good evidence to show the effectiveness of harm reduction in addressing drug-related harm, it still faces many barriers both in philosophy and practice. Harm reduction is mistakenly believed by governments to be promoting drug use. Also, due to the prevalence of abstinence-based approaches, harm reductionists find it difficult to work effectively with current drug law enforcement systems (because these systems fundamentally undermine their work), and they struggle against inadequate funding to scale up harm reduction interventions. These challenges are commonplace around the world, Vietnam included. Therefore, it is important to understand the key principles of harm reduction and to consider how this perspective might be practically implemented, especially in Asian countries like Vietnam where drug use is historically and socially viewed as a serious social problem.

The key argument of this chapter is that harm reduction is more effective than abstinence in responding to drug use problems. Harm reduction is a pragmatic and long-term approach to drug use, because it not only addresses public health issues around people who use drugs, it promotes human rights, and also contributes to achieving immediate and attainable goals like 
employment, education, or reducing stigma and discrimination. Lessons from drug reform changes in the UK, Portugal, the Netherlands, Switzerland or Canada and the US legalisation of cannabis can give some confidence for other countries to step away from criminalisation and experiment with some types of drug reform.

Philosophically, the pursuit of forced and imposed abstinence-based approaches conflicts with authentic harm reduction strategies (See Appendix One for detailed comparisons), and pursuing both models causes contradictions in both practical interventions and outcomes. These contradictions are common within the Vietnamese context, where abstinence and pseudo harm reduction are conducted simultaneously. Vietnam may outwardly express a commitment to harm reduction, but may do so within a broader philosophy that expects lifelong abstinence and forcefully imposes it on all illegal people who use drugs. This raises issues about the viability and validity of any apparent harm reduction approach. In the next chapter, the historical and current perceptions of drug use and people who use drugs in Vietnam are analysed. These provide a basis for understanding why drug use is socially and politically constructed as a 'social evil', why those who take drugs are treated as 'outsiders' in Vietnam and why Vietnam has conducted pseudo harm reduction. 


\section{Chapter Four: Drug Use in Vietnam}

\section{Introduction}

The previous chapters have noted that while Vietnam may outwardly express a commitment to harm reduction, it does so within a broader philosophy that expects lifelong abstinence, even though harm reduction states that abstinence is not required. The confusion over drugs and drug use (considering all drug use and those taking drugs are harmful) is a significant barrier to effective harm reduction in the country. To understand why Vietnamese people have such negative perceptions and attitudes, this chapter outlines the history of drug use in Vietnam.

Drug use (mainly opium) came to be seen by the state as a national enemy from the mid-19 ${ }^{\text {th }}$ century (Quang, 2009). In particular, the colonial government used opium as a means to control Vietnamese people during wartime (1858-1975) (Nguyen, 2007). The consequences of drug use from the wars were difficult as Vietnam had to deal with a great number of users who remained dependent on drugs (Vuong et al., 2012). After Vietnam gained independence in 1975 , drug use was seen as a leftover of old regimes and as external contamination from the West (Rapin et al., 2003). Despite strict controls, drug use has remained. Indeed, as this chapter details later, drugs like cannabis, amphetamine-type stimulants (ATS) and synthetic drugs have become more popular, even though opium use remains dominant. There has also been a shift in the way people consume drugs in Vietnam, from smoking to injecting. This change, alongside the lack of needle exchange facilities, has fuelled an HIV epidemic. The prevalence of drug use and HIV infection have further exacerbated hostile attitudes towards drug use in Vietnam (Heyman, 2013).

Beyond charting this history, this chapter also examines how drugs and harm are defined within Vietnam. The current drug classification is determined by two main factors, including historical issues (drugs like opium, heroin or cannabis are associated with the colonial government) and international instruments (such as the 1961 UN Narcotic Drug Convention and the UN 1971 Psychotropic Substances Convention). These have led to a strong official approach that treats legal and illegal drugs quite differently. Accordingly, within Vietnam, harms are generally seen to only be caused by illicit drug use and those taking these drugs. 
These perceptions of drugs and harm have fuelled an abstinence (rather than a harm reduction) philosophy. From a Vietnamese perspective, definitions of 'harm' generally overlook the complexity of drug use, or the role of social, environmental and legal factors that can cause more harm than the drugs themselves (Buchanan, 2015b; Buchanan \& Young, 2000; Denning \& Little, 2011; Levy, 2014). Importantly, these negative social perceptions of drug use are led by current legal classifications, but they are also based on social and political constructions, as in other countries. This chapter demonstrates that the unfavourable attitudes to drug use in Vietnam have historically served political purposes. It has been a response of Vietnam politicians to external influences (from colonial forces to increasing market neoliberalism). Also, the Vietnamese media and political discourse have influenced and shaped how Vietnamese people perceive drug use and those who take drugs. These above factors provide a basis for understanding why drug use is socially and politically constructed as a 'social evil' and why those who take drugs are treated as 'outsiders' in Vietnam.

\section{Negative Perceptions of Drug Use from History}

Situated close to the 'Golden Triangle' region, and lying across important South-East Asian region traffic routes (Laos, Myanmar and Thailand), Vietnam has a long history of producing and consuming opium. As noted in Chapter One, opium was first introduced to Vietnam around the 1600s in the northern mountainous areas before spreading to the whole country over the next centuries (Nguyen \& Scannapieco, 2008). This section illustrates some key historical milestones that generally explain why Vietnamese people have negative views towards illicit drug use. These milestones are important to understand why Vietnam has engaged one of the most punitive approaches to drug use in the world.

At first, Vietnamese people viewed opium as a medicine that could treat diseases, such as rheumatism or intestinal diseases, and relieve pain $(\mathrm{Vu}, 2000,2007)$. Yet they also recognised opium's potential for harm and addiction, and viewed it as a social evil (Vu, 2007). In order to prevent the expansion of opium cultivation and opium addiction, the first national regulation forbidding opium cultivation was promulgated by Kinh 'Canh Tri III' of the feudal state called 'Dai Viet' in 1665 (Vu, 2000). This first prohibitive regulation elaborated that: 
... men and women use opium to satisfy their libido, thieves use it as a precursor to steal other people's property ... opium use also causes fires and bankruptcies ... it severely damages people's health and those who take it are not as normal persons ... therefore, from now all mandarins and citizens have to stop cultivating and trading opium ... and for those who are growing or possessing opium, they have to demolish it immediately (cited in Vu, 2007, p. 13).

Although the feudal states outlawed opium consumption and also issued policies against opium trafficking from China, opium use still spread into other highland and lowland regions (Lau, 1993). However, the efforts of the feudal states generally failed because of the annexation of Vietnam by France in 1858 (Quang, 2009). Shortly after this invasion, the newly installed colonial government established a monopoly regulating the production and sales of opium in Vietnam in order to finance the heavy initial expenses of colonial rule (Nguyen \& Scannapieco, 2008).

In 1899 , the colonial state reorganised the opium business by scaling up sales and significantly reducing prices to make opium more affordable to poor people (McCoy, Read, \& Adams, 1972). By 1900, the tax on opium had contributed to more than $50 \%$ of all colonial state revenue (Edington, 2016). More dens and shops were established to meet increased demand and, by 1918 , there were 1,512 dens and 3,098 retail shops selling and distributing opium in Vietnam (McCoy et al., 1972). Although there was a vigorous international crusade again the 'evils of opium', which forced colonial administrations to reduce opium business elsewhere, French officials continued to expand the opium trade. By the early 1940s, it was estimated that around $2 \%$ of the Vietnamese population used opium (Vuong et al., 2012). At that time, opium was not only seen as one of the main financial pillars of colonialism, but as a powerful symbol of French intervention and oppression (McCoy et al., 1972).

After Vietnam gained independence from France in 1945, following the first Indochina War, President Ho Chi Minh linked the opium monopoly to the systematic poisoning of the Vietnamese people, criticising the French colonialists 'acting against principles of humanity and justice ... [who] had forced the use of opium on the people to weaken our race' (Edington, 2016, p. 2). He requested the strict prohibition of opium use and emphasised that the prevention of opium smoking - a 'social evil' and a remnant of the old regime - was an urgent 
task of the new government. However, the new government faced a dilemma in fully eradicating opium production and consumption (Nguyen, 2008). While considering these drug activities as expressions of colonial exploitation that were 'incompatible with new Vietnam's socialist lifestyle' (Edington, 2016, p. 2), the state had to consider their role in the socioeconomic reality of highland areas. This tension led to selective opium policies. While opium cultivation and consumption were tolerated in the highlands, the new government aggressively sought to restrict opium production and consumption in all other regions (Bankoff \& McCoy, 2007; Rapin et al., 2003). These selective drug policies were prolonged during the 'second Indochina War' (1954-1975).

Unfortunately, because the country was at war with America, no precise information or documentation is available in terms of how many people smoked opium and other drugs, how prevalent drugs were, or how drug production took place during this period in North Vietnam (Rapin et al., 2003). Meanwhile, in South Vietnam, heroin, cannabis and amphetamine consumption had become a severe problem, particularly among American and Vietnamese soldiers, ${ }^{29}$ who worked for the Southern Vietnamese Government (McCoy et al., 1972; Quang, 2009). By the early 1970s, it was estimated that $35 \%$ of American soldiers in Vietnam were using heroin, followed by cannabis and synthetic drugs (mainly amphetamine) with around $29 \%$ and $5 \%$ respectively (Menninger \& Nemiah, 2008). Before the end of the second Indochina War (1975), there were roughly 300,000 people who use drugs, including Vietnamese, American soldiers and citizens, within the Southern government border (Vu, 2007).

After 1975, the Vietnamese government began to send people who use drugs to rehabilitation camps with the aim of ridding them of the bad remnants of capitalism. These camps are considered to be the foundation of current compulsory treatment centres (06 centres) (Edington, 2016). The communist government deployed restrictive drug policies with the purpose of addressing 'a vestige of an inglorious past' (Edington, 2016, p. 2). Drugs were again

\footnotetext{
${ }^{29}$ Drugs were widely distributed to soldiers in order to prevent them from falling asleep, to make them lose weight and help them overcome the constant fear of guerrilla attack (Kuzmarov, 2013). Although around $20 \%$ of American soldiers became opiate addicts during the Vietnam War, the overwhelming majority desisted from opiate use after they returned home (Gossop, 2012).
} 
regarded as a leftover of imperialist power (Dao, Hirsch, Giang, \& Parker, 2013); therefore those who produced or consumed drugs were seen to have 'personal corruption' or 'external contamination' (Edington, 2016, p. 2). This rhetoric of contamination has continued, albeit in different ways, particularly given the strong emphasis on linking HIV/AIDS prevention and eradication with efforts to combat drug use (Edington, 2016). Article 61 of the 1992 Vietnamese Constitution (1992) declares drug use to be a dangerous social disease and a social evil that needs to be immediately eradicated (Windle, 2015). This legal foundation has paved the way for an intensification of a broad government campaign against drug use.

\section{Political and Social Constructions of Drug Use}

The history of drug use in Vietnam has, as shown previously, played an important role in shaping negative attitudes and perceptions toward drug use and people who use drugs. Yet alongside this history, many other factors have strongly influenced the way people perceive drug use and users, including (i) external contamination, (ii) addiction, (iii) crime, and (iv) media influences. This section illustrates how these political and sociocultural elements impact on the perceptions of 'evil' drug use in Vietnam. Importantly, this section reinforces that fact that in Vietnam, as in other countries, drug use is politically and socially constructed.

\section{External Contamination}

From 1986, under the 'Doi Moi' (Renovation) policy, Vietnam inaugurated a profound economic and social transformation (Le et al., 2013). This Doi Moi shifted the country from a centrally planned economy to one that is market-oriented. The government subsequently loosened controls on domestic and foreign goods, and opened opportunities for private businesses and foreign investment, and even foreign-owned enterprises. The country has transformed from one of the poorest nations into one of Southern Asia's most prosperous economies. Yet the Doi Moi policy has presented Vietnam with new challenges, not least as the Vietnamese government has sought to protect and consolidate its power and authority in a market-oriented economy. As Beresford (2008) argues, the Vietnamese government not only has to handle new demands arising from its population, but to grapple with aid donors and foreign investors who tend to influence the country's economic policies. Although the country has some successes regarding the rise in living standards and the reduction of poverty, the Doi Moi policy has also resulted in deepening inequalities (Edington, 2016). Some 
welfare subsidies and social safety nets, such as healthcare or educational support, have been cut off (Phinney, 2008). As a result, people who use drugs who are seen as a burden to society have inevitably encountered a conspicuous lack of social and health service support. This has remained almost unchanged to date.

With the advent of the market-oriented economy, the Vietnamese government has also tried to control the effects of market liberalisation upon national identity (Wilcox, 2000). The term 'Van Minh'30 (being civilised) is used in both official and popular discourses to manage and make sense of the local presence of the global economy (Bradley, 2004). Promoting Van Minh discourse is regarded as an attempt by Vietnam's government to strengthen national spirit, revitalise tradition and preserve national culture (Pettus, 2004). As Edington (2016, p. 4) explains:

In contemporary nationalist discourse, Van Minh rests on the articulation of a seamless Confucian past alongside the imperatives of the neoliberal marketplace and its implicit cultural dangers. Van Minh has therefore come to embody the convergence of the material and cultural ambitions of the Vietnamese postcolonial state in the forging of a national identity.

The state's campaign for attaining Van Minh was also intended to 'anchor Vietnam's timeless spiritual past as an ethical and moral counter to the forces of Western Capitalism' (Edington, 2016, p. 4). For example, drug use, prostitution, karaoke bars, massage parlours and pornography with Western models were deemed to represent 'social evils', 'dirt' and an unhealthy lifestyle, which could degenerate good 'morality' (Rydstrøm, 2006, p. 2). Drug use is clearly highlighted as a result of negative values from the West, which are considered to be opposed to original Vietnamese values (Rydstrøm, 2006). In response to the fear of external contamination (mainly drug use problems), in 1994, Vietnam's Prime Minister Vo Van Kiet requested the mobilisation of mass organisations, state bureaucracy and the public at large

\footnotetext{
30 'Van Minh' refers to the social and economic qualities in developed countries that Vietnam tries to achieve, such as political order, modern education, health standards, or economic disciplines.
} 
to exercise all 'poisonous' forces from Vietnam society, such as prostitution, drug and alcohol addiction or gambling (Edington, 2016).

Also, the externally poisonous forces are argued to cause the degradation of Vietnamese young people (Windle, 2015). That is, according to a 2015 MOLISA report, around $76 \%$ of registered people who use drugs (202,000 individuals) were aged below 35 years old, and about $60 \%$ (over 92,000) had first tried drugs before the age of 25 years (MOLISA, 2015). It was reported that young people have the highest consumption rates of illicit drugs and also legal drugs like alcohol and tobacco (Ministry of Health, 2018a). Importantly, it must be acknowledged that most of these young people do not have problems with their drug use at all (Hong et al., 2011). However, the General Secretary of the Central Committee of the Communist Party of Vietnam, Le Kha Phieu, has stressed the importance of protecting the young generation from drug use to ensure that the future decision-makers of Vietnamese society can remain non-polluted (Rydstrøm, 2006). Chan, Kerkvliet, and Unger (1999) argue that Vietnam wants to prevent social evils because they argue that, if adolescents were influenced by 'dirty' foreign forces (drugs and prostitution), the future of Vietnam would be jeopardised. Therefore, those who take drugs are regarded as having low moral standards that violate 'Vietnamese social conventions' ${ }^{\prime 31}$ and as copying negative western lifestyles (Hong et al., 2011). Nevertheless, the external factors are not only the reasons to explain negative social and political constructions toward drug use in Vietnam, as other misperceptions are also widespread.

\section{Drug Use inevitably Leads to Addiction}

The Vietnamese government and relevant agencies often propagandise that illicit drug use inevitably causes addiction. Similarly, many studies conducted by Vietnamese drug experts (Duc, 2012; Thien, 2002; Vu, 2000, 2007) argue that drug use is harmful and addictive. Indeed, the idea that using illegal drugs will lead to addiction is presented as an iron law in Vietnam (Hong et al., 2011). The common message is that using a drug even once will cause addiction

\footnotetext{
31 Vietnam is heavily influenced by Confucianism, which emphasises the significance of conventional values and personal moralities, so people who use drugs therefore have been viewed as weak-willed and morally corrupted individuals or 'social evils' (Edington, 2016; Hayes-Larson et al., 2013).
} 
(Thien, 2002). The conflation between drug use and addiction is such that many families and communities think that all people who use drugs must be sent to treatment centres for detoxification (Hong et al., 2011). This confusion has resulted in the widespread use of abstinence-based approaches like compulsory treatment centres. As Nguyen and Scannapieco (2008) explain, from a Vietnamese perspective, addiction is largely connected to individual biological or psychological defects.

Accordingly, solutions or interventions against addiction are generally placed at the level of the person, rather than towards social and environmental issues (further discussed in Chapter Five). In Vietnamese society, whenever people see or hear of someone taking drugs, people will view them as 'addicts'. This stereotype is widespread because Vietnamese official materials and propaganda campaigns frequently make a claim that people use drugs once and you're addicted (USAID, 2011). This message is widely disseminated across the whole country with the purpose of influencing people to stay away from drug use. It is evident that the Vietnamese construction of drug use reflects the dominance of the moral model of addiction, which partly explains why Vietnam has a punitive approach to drug use. Nevertheless, constructing drug use as inevitably causing addiction has not stopped people from using drugs, as drug use in Vietnam has increased, as noted previously. As elsewhere, ${ }^{32}$ these campaigns have increased stigma and discrimination.

\section{Drug Use Causes Crime}

In addition to claiming drug use leading to addiction, the Vietnamese government, MOLISA and MPS often emphasise that illicit drug use causes crime or at least is responsible for any increase in the crime rate (Nguyen, 2002). For example, MOLISA (2015) argues that a large proportion of arrestees' criminal activity (over 70\%) is attributable to problematic drug use. This type of constructed evidence is used to cement an inevitable connection between drug

\footnotetext{
32 For example, in 2005, the Montana Meth Project was implemented in the US with the aim of reducing methamphetamine use. Their by-line was 'meth, not even once'. However, as Erceg-Hurn (2008) argues, the campaign exacerbated the risks of methamphetamine use and in general had no impact on the drug use trends in Montana. Ironically the project increased disapproval of people taking drugs among Montana citizens and increased social stigma against people who use drugs.
} 
use and crime, or to put it another way, drug use turns law-abiding citizens into criminals who commit crime to obtain drugs (Nguyen, 2002). Yet Vietnam fails to distinguish which mechanism or motive leads Vietnamese people who use drugs to criminal behaviours. For instance, Vietnam does not analyse how many people committed crimes under the effects of drug use, how many became offenders to fund their drug habit, or how many had criminal behaviour as a result of inequality, a lack of social capital and social exclusion. Vietnam also never considers a systemic model of the drug use and crime nexus, which was developed by Goldstein (1985). Attacking the political system/prohibition as a source of crime is extremely sensitive in the country. Blaming drug use for causing crime has dominated the coverage of the Vietnamese media and it is embedded in the general public's mind-set. For example, as noted previously, ANTV, a popular television channel in Vietnam, often bombards people with sensational headlines like 'drug use and addiction is the shortest path leading to criminal behaviours' (Dinh \& Duc, 2014, p. 1).

Furthermore, it is really important to note that the Vietnamese government only counts those who have been arrested/incarcerated and does not record all those people who use drugs who are engaged in recreational drug use. As discussed in Chapter Two, using only those arrested to argue drug use 'causes' crime is contested. As Bean (2014) and Wilkins et al. (2015) argue, most arrestees are unproblematic drug users. Although there is a number of problematic drug users, they only represent a small minority of the drug use population. Unfortunately, these arguments are not taken into serious consideration in Vietnam. The misperception of the drug use-crime connection has reinforced the common view that there is an inevitable link between drug use and criminal behaviour. This perception also has a strong link with the brain and moral models of addiction in which drug use and users are seen as the drivers of social disorder and crime. Therefore, enforced abstinence-based approaches, such as punishment, incarceration or detention, are preferred methods for preventing crime. As noted above, challenging Vietnam's tough enforcement/prohibition measures is extremely difficult for any Vietnamese researchers. Like elsewhere, the common stereotypes of the contested drug-crime connection still dominate Vietnamese drug policy and the media. 


\section{Media Influence}

The Vietnamese social and political constructions of drug use are also accompanied by Vietnamese media campaigns, which stereotype drug use as a serious social problem and present people who use drugs as weak-willed and morally corrupted individuals, as blights on the honour of their family and the country as the whole (Edington, 2016). To propagandise drug use and people who use drugs, the Vietnam government has often used caricatures showing the skull and crossbones to indicate the connection between drug use, people who use drugs and the HIV epidemic (Kincaid \& Sullivan, 2010).
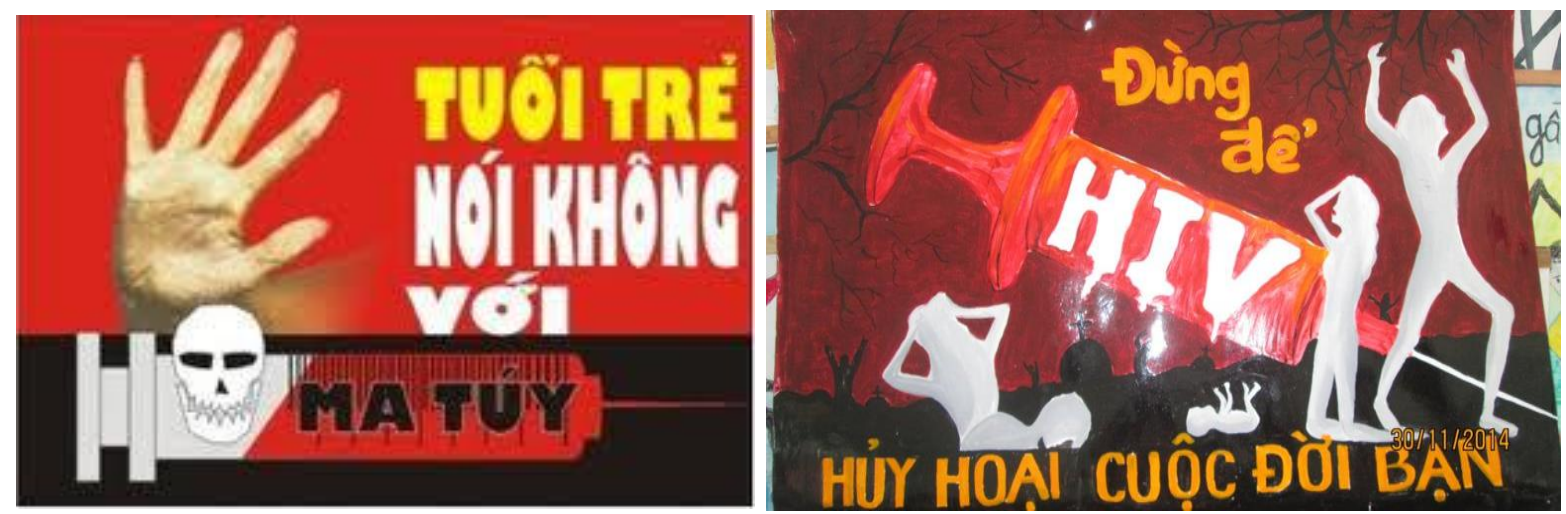

Image 4.1 (Left): Young people say no to illicit drugs (The National Committee on AIDS Drugs and Prostitution Prevention and Control 2011)

Image 4.2 (Right): Do not let drug use and HIV destroy your life (The National Committee on AIDS Drugs and Prostitution Prevention and Control 2011)

These images cement the fact that drug use is a social evil, not really a 'public health issue'. ${ }^{33}$ As Taylor (2008, p. 374) argues, caricatures that portray drug use in the media generally provide:

\footnotetext{
${ }^{33}$ Since the early 2000s, the perception of drug use and addiction has gradually shifted from a social evil problem to a public health issue, on paper. For example, the 1999 Penal Code removed the criminalisation of personal possession, which provided a basis for considering drug use as a public health matter instead of a criminal one. In 2006, the HIV Prevention Law officially acknowledged that drug use and drug addiction are public health issues. However, in practice, drug use has been seen as an administrative violation in the 2012 Administrative Violation Law - which means that people who use drugs are still cast as a high risk group that potentially cause social disorder or crimes (Ahmed et al., 2014). Therefore, drug use and users are largely seen as social evils rather than a health issue and patients. Apparently, while social evil might be ousted from code, it remains a significant issue in terms of political/media/social approaches.
} 
... a simplistic understanding of the situation and reinforces the belief that something can be done realistically and relatively easily about the drugs crime 'problem' if you stop drug use amongst 'this' group via the criminal justice system.

The media frequently exacerbate the truth about drug use to capture the attention of the public and politicians. For example, in 2009, the UK newspapers, such as the Sun, the Guardian or the Independent newspapers reported on a drug called 'mephedrone' (4methylmethcathinone or street names: meph, meow meow or topcat) for causing fatal accidents, and an immediate ban on this drug was imposed (Alexandrescu, 2014; Lancaster, Hughes, Spicer, Matthew-Simmons, \& Dillon, 2011). To emphasise the danger of mephedrone, the Sun published a sensational headline titled 'Legal drug teen ripped his scrotum off' (Alexandrescu, 2014, p. 23), which described how mephedrone ruined a teenager. However, the story was a fake. The problem here is that the UK government banned this drug based on the media reports rather than clinical and pharmacological research about mephedrone (Alexandrescu, 2014). This drug was legally sold as plant fertiliser and had no history of observed clinical side-effects (Nutt, 2012). The example of mephedrone reaffirms that the media often exaggerates and uses inaccurate information about drug use to draw attention. Instead of helping the community, the exacerbated and inaccurate information about mephedrone actually increased its use (Lancaster et al., 2011; Nutt, 2012; Ritter et al., 2011).

In Vietnam, the government continues to use negative caricatures stereotype that drug use is a social evil and that people should avoid this high-risk behaviour (Nguyen \& Scannapieco, 2008). Almost every day Vietnamese people are bombarded with breaking news and sensational headlines that generally associate people who use drugs with deviant or criminal behaviours to attract public attention. For example, Vietnamese news often mentions people who use drugs as follows:

Robberies are increasing for many reasons, but the dominant reason is the high number of drug addicts ... Many people who use drugs form gangs that get high before going out on the street to rob - Lieutenant General Trieu Van Dat of the Ministry of Public Security (Cited in Dinh \& Duc, 2014, p. 1). 
The ongoing campaign to collect drug addicts into rehabilitation centers in $\mathrm{Ho}$ Chi Minh City has helped improve the crime situation in the city remarkably - Senior Lieutenant Colonel Nguyen Si Quang (Cited in TuoitreNews, 2014, p. 2).

Police have warned about increasing cases of people committing crimes under the influence of methamphetamine, especially "ice" (crystal methamphetamine), as the drug trade has grown in recent years - Major General Nguyen Anh Tuan, Director of the Drug-Crime Police Department at the Ministry of Public Security (Cited in Dinh \& Duc, 2014, p. 1).

Doan Trung Dung, the prime suspect in the killings of a sexagenarian woman and her three grandchildren in Uong Bi City, Quang Ninh, has been arrested ... According to the suspect's initial statements, he admitted the crime, confirming that he was high on 'crystal meth' (Cited in TuoitreNews, 2016, p. 3).

Ironically, the same representations are generally not applied for other legal drugs like alcohol or tobacco. That is, alcohol and tobacco are treated differently, despite the fact that they cause more extreme 'harms' than illicit drugs, such as cannabis or ecstasy (Nutt et al., 2010), particularly in Vietnam (further detailed later). As in other countries, drug taking in Vietnam is influenced by factors such as choice, peer pressure, boredom, or social networks (Ministry of Health, 2016b). According to the Ministry of Health (2016b) report, about $80 \%$ of Vietnamese males use alcohol, while the figure for females is around $37 \%$. Regarding tobacco use, about $17 \%$ of Vietnamese people have smoked cigarettes (Vietnam Steering Commitee on Smoking and Health, 2016). Alcohol and tobacco use has led to many health, social and economic problems in Vietnam, but their use is socially and culturally accepted (Ministry of Health, 2016b). It is often ignored within government discourses that alcohol use results in crime, violence and domestic violence in Vietnam (Ministry of Health, 2016b). Nevertheless, as explained by Gossop (2012), legal drugs are generally accepted because they are seen to be (relatively) safe and, importantly, they are good businesses. The inaccurate representations for legal and illegal drugs are, as noted previously, based on social and political factors rather than evidence and rationale. 
In short, political and sociocultural elements have a strong impact on the perceptions of 'evil' drug use in Vietnam, and how drug use is politically and socially constructed in Vietnam. Yet the history and contemporary reality of drug use is more complex, and it is important to move beyond the overly simplistic axiom that drug use causes addiction, crime and social problems (Bennett \& Holloway, 2005; Boland, 2008; Holloway et al., 2005). The use of drugs for recreational, medical and social purposes is recorded throughout human history and only a small number of those who take drugs become problematic users (Buchanan, 2006, 2009). In fact, according to UNODC (2015) statistics, between 2006-2013, problematic illicit people who use drugs only accounted for over $10 \%$ of global illicit people who use drugs. The rest are recreational people who use drugs who do not resort to crime or other anti-social behaviours (Allen, 2005; Bean, 2014; Bennett \& Holloway, 2005; Buchanan, 2015b; Buchanan \& Young, 2000). This fact generally is ignored in Vietnam. Vietnamese researchers, the media and government have exaggerated the connection between drug use and crime, addiction and the perils of drug use, and have limited the discussion of the complexity of drug experiences and perpetuated inaccurate stereotypes for political purposes rather than evidence. Although Vietnam has made great efforts to prevent and warn people from using illicit drugs, the contemporary drug situation has remained complicated.

\section{Contemporary Drug Use Problems}

The problems of drug use in Vietnam are, as noted above, long-standing. This is primarily due to Vietnam's position near the 'Golden Triangle' and across important South-East Asian trafficking routes. As a result of market-oriented policies, foreign trade has also been liberalised, and 'foreign culture' has become popular in Vietnam. This social and economic change has arguably increased illicit drug use, especially among young people influenced by global cultural trends (Quang, 2009).

Over time, Vietnam has also shifted from a transit country to being both a transit and drugconsuming country (Vuong et al., 2016). The country has witnessed a significant increase in the number of 'people who use drugs' ${ }^{34}$ For instance, between 1994 and 2004, the number

\footnotetext{
${ }^{34}$ In Vietnam, people who use drugs refer to all users (including PWID) who test positive for drugs (listed in Vietnam's 2006 Drug Control Law) and are supervised by authorities. Another term for people who use drugs is registered people who use drugs. A philosophical
} 
of people who use drugs nearly tripled, from around 60,000 users in 1994 to over 170,000 users in 2004 (Nguyen, Pauline Oosterhoff, Yen Pham Ngoc, Pamela Wright, \& Hardon, 2009). In 2014, there were around 204,000 people who use drugs in the country, and the figure for 2017 was about 211,000 users among a population of almost 93 million people (Luong, 2017). The actual numbers are likely to be much higher, since many users do not want to disclose because of the risk of police harassment and incarceration in compulsory treatment centres (Rapin et al., 2003; Windle, 2015). Vuong et al. (2012) estimate that the actual figure could be over 500,000 .

In 2012 , the majority ${ }^{35}$ of people who use drugs (at $84.7 \%$ ) were heroin users, followed by $6.5 \%$ for synthetic people who use drugs, $6.4 \%$ for opium users, $1.6 \%$ for cannabis users and $0.3 \%$ and $0.5 \%$ for pharmaceutical and other drugs respectively (Tettey et al., 2013, p. 136). There have been remarkable changes in recent years. For instance, in 2015, it was reported that although heroin use was still dominant among Vietnamese people who use drugs (accounting for 75\%), the prevalence of amphetamine-type stimulants (ATS) and cannabis use increased to $11.5 \%$, and $3.7 \%$ respectively, while opium use dropped to $4.2 \%$ (UNODC, 2017). Table 4.1 below illustrates how drug use trends have recently changed in Vietnam (20112015) based on the reports of the UNODC.

commitment to the eradication of social evils during the 1990s also promoted punitive approaches to drug use (Kincaid \& Sullivan, 2010) - intense government campaigns have criminalised drug consumption and formalised forced treatments for perceived 'addiction' (Huong, 2002). Periodic crackdowns against users, mandatory detention and forced detoxification have been conducted across the country (Edington, 2016).

${ }^{35}$ Due to the prevalence of heroin in Vietnamese society, heroin users are a police focus. It is also expected that this is an indication of the policing, control, and regulation of users, rather than being an indication of actual drug use in Vietnam. 
Table 4.1: Trend in use of selected drugs in Vietnam, 2011-2015 (UNODC, 2017, p. 50)

\begin{tabular}{|l|c|c|c|c|c|}
\hline Drug type & 2011 & 2012 & 2013 & 2014 & 2015 \\
\hline Crystalline methamphetamine & $\uparrow$ & $\uparrow$ & $\uparrow$ & $\uparrow$ & $\uparrow$ \\
\hline Methamphetamine tablets & $\uparrow$ & $\uparrow$ & $\uparrow$ & $\uparrow$ & $\uparrow$ \\
\hline Methamphetamine powder & $\bullet$ & $\uparrow$ & $\uparrow$ & $\uparrow$ & $\bullet$ \\
\hline Ecstasy & $\uparrow$ & $\uparrow$ & $\bullet$ & $\leftrightarrow$ & $\bullet$ \\
\hline Cannabis herb & $\downarrow$ & $\uparrow$ & $\uparrow$ & $\leftrightarrow$ & $\uparrow$ \\
\hline Heroin & $\downarrow$ & $\downarrow$ & $\downarrow$ & $\uparrow$ & $\uparrow$ \\
\hline Ketamine & $\bullet$ & $\bullet$ & $\bullet$ & $\bullet$ & $\bullet$ \\
\hline Opium & $\downarrow$ & $\downarrow$ & $\uparrow$ & $\uparrow$ & $\bullet$ \\
\hline
\end{tabular}

$\uparrow=$ Increasing, $\downarrow=$ Decreasing, $\leftrightarrow$ = Stable,$\bullet=$ Not reported

The use of amphetamine-type stimulants (ATS) (amphetamine and methamphetamine) and cannabis has increased in Vietnam. For example, ATS users accounted for $90 \%$ of newly registered people who use drugs in some southern provinces in the country in 2015 (UNODC, 2017). Regarding cannabis, exact data on its prevalence has not been available, but cannabis use is dominant in Vietnam, according to UNODC (2016a). For example, Dinh and Duc (2014) reported that $80 \%$ of people who use drugs in Vietnam had tried 'weed' (cannabis). Also, the amounts seized by Vietnamese police further illustrate the significant increase in cannabis and ATS use in Vietnam as a result of increased police crackdowns. Table 4.2 below shows the seizures of selected illicit drugs in Vietnam during 2011-2015, cannabis and ATS included.

Table 4.2: Seizures of selected drugs in Vietnam, 2011-2015 (UNODC, 2017, p. 51)

\begin{tabular}{|c|c|c|c|c|c|c|}
\hline Drug type & Measurement & 2011 & 2012 & 2013 & 2014 & 2015 \\
\hline ATS & tablets \& kg & $\begin{array}{l}366,000 \\
\text { tablets }\end{array}$ & $\begin{array}{c}335,470 \\
\text { tablets and } \\
129 \mathrm{~kg}\end{array}$ & $\begin{array}{c}332,772 \\
\text { tablets and } \\
118 \mathrm{~kg}\end{array}$ & $\begin{array}{c}297,285 \\
\text { tablets and } \\
352 \mathrm{~kg}\end{array}$ & $\begin{array}{c}696,632 \\
\text { tablets and } \\
983 \mathrm{~kg}\end{array}$ \\
\hline Cannabis herb & $\mathrm{kg}$ & $\begin{array}{c}7 \mathrm{mt} \text { of } \\
\text { 'fresh' and } \\
500 \mathrm{~kg} \text { of } \\
\text { 'dried' }\end{array}$ & $\begin{array}{c}1 \mathrm{mt} \text { of } \\
\text { 'fresh' and } \\
164 \mathrm{~kg} \text { of } \\
\text { 'dried' }\end{array}$ & $\begin{array}{l}932 \mathrm{~kg} \text { of } \\
\text { 'fresh' and } \\
225.7 \mathrm{~kg} \text { of } \\
\text { 'dried' }\end{array}$ & 422 & 4,500 \\
\hline Cocaine & $\mathrm{kg}$ & $\bullet$ & $\bullet$ & 2.4 & 42.6 & 178 \\
\hline Heroin & $\mathrm{kg}$ & $\begin{array}{l}309.0 \mathrm{~kg} \text { and } \\
36 \text { 'blocks' }\end{array}$ & 692.0 & 940 & 922 & 1,510 \\
\hline Ketamine & $\mathrm{kg}$ & $\bullet$ & $\bullet$ & $\bullet$ & $\bullet$ & $\bullet$ \\
\hline Opium & $\mathrm{kg}$ & 76.0 & 225.0 & 117 & 32 & 133.8 \\
\hline
\end{tabular}


Over the past two decades, there has been a shift away from smoking to injecting drugs in Vietnam, as within other Asian countries (Edington, 2016; Windle, 2015). Prior to the 2000s, a large majority of people who use drugs (over 85\%) preferred smoking opium; however, later on, smoking declined in popularity and injecting heroin became the most popular way of consuming illicit drugs in Vietnam among registered people who use drugs (MOLISA, 2010). There are two main reasons for the shift from opium smoking to injecting heroin. Firstly, heroin began to dominate local drug markets (following the trend in South East Asian region regarding drug use) in the late 1990 s with a sharp increase in the use of injection as a method for self-administering (Nguyen \& Scannapieco, 2008). Secondly, injecting heroin is much cheaper than smoking heroin (Ahmed et al., 2014). Injection has continued to remain popular among Vietnamese people who use drugs because intravenous crystal methamphetamine consumption has increased in popularity, as in other Asian countries (Windle, 2015).

The shift from drug smoking to injecting has been regarded as the principal driver of the HIV epidemic in Vietnam. As noted earlier, the first case of HIV infection was reported in Ho Chi Minh City (HCMC) in 1990 (Maher et al., 2007; Nguyen \& Wolffers, 1994). In 1992, it was estimated that around 3,000 persons were living with HIV and over $85 \%$ of them were injecting drug users (IDUs) (Ministry of Health, 2006). Later, Vietnam experienced a rapid spread of HIV infection both among the general Vietnamese population and among IDUs. By 1999, HIV infection had been recorded in all provinces and cities (VAAC, 2011) and ten years later, in 2009 , IDUs accounted for $44 \%$ of HIV cases, and over $50 \%$ of newly detected HIV cases (Tran, Ohinmaa, Duong, Do, Nguyen, Mills, et al., 2012). The increases of HIV infection among people who use drugs are because many users are too scared to access health services, where they could be arrested and then detained in treatment centres (Hong et al., 2004). This affirms that Vietnam's punitive policies have exacerbated rather than reduced harm.

Although the HIV prevalence among IDUs has recently lowered due to the development of needle exchange programmes - dropping from over 13\% in 2012 to around $9 \%$ in 2015 (Ministry of Health, 2016a, 2016b, 2018a) - this does not confirm an absence of risk behaviours (Ahmed et al., 2014). For example, in 2014, it was reported that in some provinces - located in the north (Hai Phong, Bac Ninh, Nghe An), northwest (Dien Bien, yen Bai), northeast (Quan Ninh), Hanoi City and Ho Chi Minh City -- the frequent practice of sharing 
injecting equipment is widespread (around 30\% among IDUs) (Ahmed et al., 2014; Nguyen \& Scannapieco, 2008; Windle, 2015). In addition, Vietnam has retained a higher level of HIV prevalence among its population (at $0.53 \%$ ) compared to nearby countries such as China (0.23\%), Myanmar (0.22\%), India and the Philippines (both only 0.02\%) (Bergenstrom, Andreeva, \& Reddy, 2013).

In general, Vietnam has witnessed a significant increase in drug consumption and the number of people who use drugs. Opium and heroin are still dominant, but other drugs like cannabis, synthetic drugs, intravenous crystal methamphetamine and ATS have gradually become more popular. Following regional trends in South East Asia, injecting (mainly heroin) has replaced opium smoking to become the most common way to consume illicit drugs in Vietnam. This shift is seen to have fuelled the HIV epidemic in Vietnam. From the early days of the HIV crisis, there has been a strong emphasis on linking HIV/AIDS prevention or eradication with efforts to combat drug use (Edington, 2016). Therefore, apart from historical reasons, the HIV epidemic has contributed to negative attitudes towards illicit drug use and people who use drugs in Vietnam (Windle, 2015). These attitudes have been further consolidated by how the Vietnamese government defines drugs and harm, to which this chapter now turns.

\section{Definition of 'Drugs' and 'Harm'}

The Vietnamese translation of the word 'drug' is a Chinese-Vietnamese compound word: 'Ma Tuy' ( $\mathrm{Vu}, 2000)$. ' $\mathrm{Ma}^{36}$ refers to something that paralyses people, while 'Tuy' means something that causes people to get drunk. The term 'drugs' means 'illicit drugs' or 'narcotics' in the Vietnamese context. Ma Tuy is widely used to refer to harmful substances like heroin, opium or methamphetamine, but it does not refer to harmful substances like alcohol or tobacco. The term Ma Tuy is apparent within social life but also within Vietnamese laws, guidance or other legal documents (Nguyen, 2008). The meaning of the word Ma Tuy is very negative (referring to something that destroys people), and it generally ignores the positive implications of drug use, such as their medical benefits or recreational use. For instance,

\footnotetext{
${ }^{36}$ This also means ghosts in Vietnamese language. Although Vietnamese professionals do not use this meaning to explain the term 'Ma Tuy' in official documents, those using illicit drugs (Ma Tuy) are seen as 'ghosts' (or remnants of their former selves) by ordinary Vietnamese people.
} 
according to the Vietnamese dictionary, Ma Tuy refers to all substances that cause people to feel ecstatic or drowsy, and that will cause dependency if people use them many times ( $\mathrm{Vu}$, 2007). Some positive implications of Ma Tuy or 'narcotics', such as reducing pain or providing pleasure, in particular, are generally overlooked by Vietnamese people. Many governments, including Vietnam, tend to ignore discourses of pleasure - the heart of liberalism - because they worry that these discourses can foster drug consumption (O'Malley \& Valverde, 2004).

According to the current penal code (the 2009 Criminal law), Ma Tuy refers to narcoticcontaining plants (including the opium poppy, the coca bush, the cannabis plant or other plants containing narcotic substances), heroin, cocaine, and other substances in liquid or solid form. These narcotic substances are socially and politically constructed pseudo-scientific terms (as discussed in Chapter Two). Narcotics are claimed as causing drowsiness or sleep (Nutt, 2012), so alcohol and opioids are also narcotic drugs. Meanwhile, cocaine, methamphetamine, cannabis, LSD are not. Also, language plays an important role in explaining why Vietnamese people see illicit drugs as negative while other drugs like alcohol and tobacco are regarded in neutral ways, or socially and culturally embraced within Vietnamese society. For example, alcohol is labelled 'Ruoi', tobacco is named 'Thuoc La', while medicines are called 'Thuoc'. In practice, alcohol (Ruoi) and tobacco (Thuoc La) have neutral meanings, while medicine (Thuoc) has positive implications (treating illnesses and saving people) (Ministry of Health, 2016b). Although alcohol (Ruoi) and tobacco (Thuoc La) are seen as harmful from the government's perspective, they are socially and culturally adopted as normalised drugs (Ministry of Health, 2016b).

In reality, alcohol and tobacco have caused plenty of harm to Vietnamese society. For instance, the Ministry of Health (2016b) reports that there are, on average, around 44,000 fatalities related to alcohol and 4,000 associated with tobacco per year, while the figure for drug-related fatalities is about 10,000 (3,000 overdoses and 7,000 associated with HIV and other blood-borne diseases). Notably, in 2012, it was reported that Vietnamese people spent over US\$3 billion on beer and alcohol while, ironically, Vietnam - an agricultural country only made around US\$3.5 billion (gross) from rice exports (Ministry of Health, 2016b; VTV, 2013). In Vietnam, alcohol and tobacco cause more problems than illicit drugs, but this seems 
to be ignored within Vietnamese academic and political debates in common with other countries (Ministry of Health, 2018a).

All illicit drugs or narcotic drugs in Vietnam are regulated in the 2006 Drug Control Law (see Appendix Two). This law divides drugs into four schedules:

(i) Schedule 1 contains the 'extremely dangerous narcotic substances' which are strictly prohibited, such as cannabis, heroin, and MDMA (ecstasy) or morphine methobromide;

(ii) Schedule 2 refers to toxic substances that are limited to use in medical therapy or scientific studies, such as amphetamine, methamphetamine, methadone, cocaine, morphine, or opium;

(iii) Schedule 3 covers the stimulants that can be used in medicine, science or experiments, including ketamine, bromazepan, buprenorphine or triazolam;

(iv) Schedule 4 encompasses the precursor substances used for preparing synthetic drugs, including addictive and psychotropic substances, such as acetic acid, acetone, pseudoephedrine (PSE) and ephedrine (substances to produce synthetic drugs).

These Vietnamese schedules have been driven by and are based on outdated drug classifications that have emerged from the UN's 1961 Narcotic Drug Convention and the 1971 Psychotropic Substances Convention. Under the influence of these instruments, Vietnam calculates heroin, cannabis, or LSD as the most dangerous drugs, followed by amphetamine, opium, cocaine or methadone. As shown in previously discussed research by Nutt and colleagues $(2007,2010,2012)$, this Vietnamese drug classification is inaccurate and ignores scientific evidence in terms of the harms caused by drug use. One obvious example is that alcohol and tobacco are the harmful drugs, but they are not prohibited under the Vietnamese drug schedules, while LSD and buprenorphine are among the least problematic (according to Nutt et al. (2010)) but are categorised as Schedule 1 and 2 respectively (see Figure 4.1). Similarly, while cannabis is decriminalised in countries such as the Netherlands or Portugal (Buchanan, 2015b; Nutt, 2012; Nutt et al., 2010), it is classified in Schedule 1 as one of the most dangerous drugs in Vietnam. Figure 4.1, developed by David Nutt and colleagues, illustrates the overall harm caused by the most popular drugs. 
Figure 4.1: Drugs ordered by their overall harm scores (Nutt et al., 2010; Nutt, King, Saulsbury, \& Blakemore, 2007)

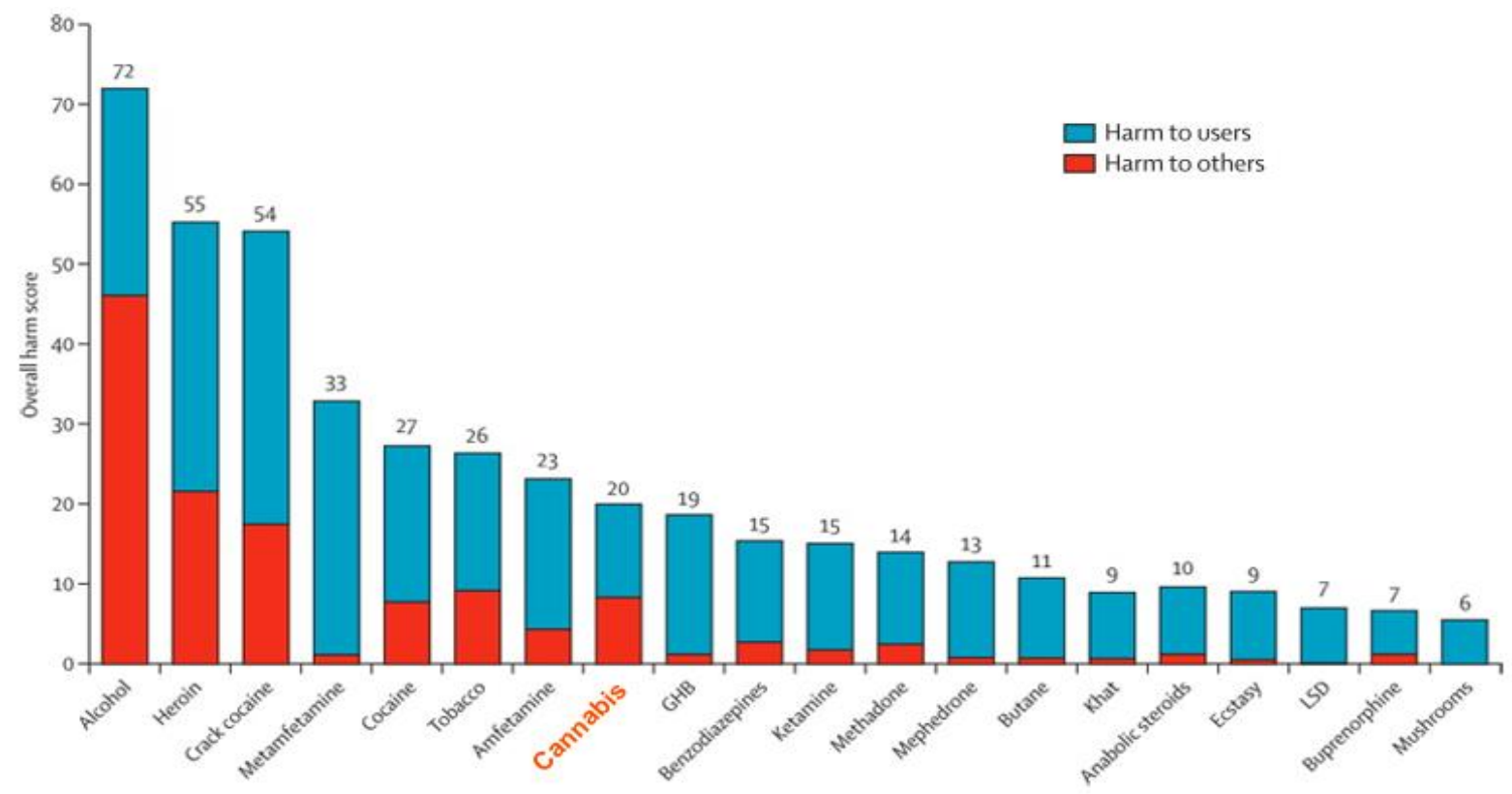

The bifurcation of Vietnamese drug classification (into legal and illegal drugs) over-simplifies the complexities of present-day drug consumption. For instance, Buchanan and Young (2000, p. 410) argue that the separation of legal and illegal drugs is misleading:

The consumption of legal and illegal drugs for pleasure should be recognized as a highly complex social issue, but instead it has been presented within a reductionist framework. Within certain boundaries the government sees the use of legal drugs (primarily alcohol and tobacco) as wholly acceptable, whereas the use of illicit drugs in any circumstance is seen as dangerous and harmful.

It is evident that heroin, opium or cocaine are addictive drugs, but this is also the case for legal drugs like alcohol and tobacco (Nutt, 2012; Nutt et al., 2007). In fact, Nutt et al. (2010) indicate that smoked tobacco is the most addictive commonly used drug, followed by heroin and alcohol. Furthermore, as discussed previously, alcohol and tobacco addiction cause more death and health problems than illegal drug addiction not only in Vietnam (Ministry of Health, 2016a), but in other countries too (Boland, 2008).

As a consequence of drug definitions and classification, Vietnamese government officials and policy-makers have presented a narrow and misleading perception of harms related to illicit 
drugs. Vietnamese authorities tend to frame harm as being caused by illicit drugs or illicit people who use drugs. Similarly, many Vietnamese researchers like Vu (2007) or Thien (2002) and key stakeholders, namely MOLISA (2015), the Vietnamese Government (2014) or even VAAC (2011) often see harm as being caused by the ravages of drugs and their inherent psychoactive properties, which lead to brain disease (addiction) and danger. This perception of harm in Vietnam is not unique. The International Network of People Who Use Drugs (2014) even emphasises the physical harms of drugs and drug use, such as addiction, blood-borne diseases, the higher risk of non-fatal and fatal overdoses, or a greater chance of premature death. In Vietnam, in general, there are no distinctions made between problematic drug use and recreational drug use, so all illegal drug use is seen as harmful and everyone who uses illegal drugs is referred to as being addicted to drugs, which is generally not applied to legal drugs like alcohol or tobacco. Therefore, illicit people who use drugs are policed and controlled for their drug use and they are also seen to be the main bearers of harm for Vietnam society.

From these approaches, Vietnamese drug law enforcement agencies have advanced the idea that reducing harm means preventing illegal drug use, controlling people who use drugs and importantly promoting a so-called drug-free society (Vuong, 2016). In reality, the Vietnamese government tends to exacerbate the danger of harm from illicit drugs in order to prevent the escalation of drug use and importantly to control and punish people who use drugs (Windle, 2015). The Vietnamese representations of harm categorised by the Vietnamese government are generally misleading and not necessarily based on evidence, science or rationality. Representations of harm are also far removed from that found in authentic harm reduction philosophies, resulting in differences in implementing harm reduction in Vietnam compared to other countries, such as the Netherlands, Switzerland or Canada. Further, only considering harm from the harmful effects of drugs generally ignores the fact that many harms are actually caused by prohibitionist policies, not the drugs themselves, and such harms would be evident if governments treated alcohol or tobacco in the same way (Buchanan, 2009).

In short, Vietnamese definitions of 'harm' generally overlook the complexity of drug use, and the role of social, environmental or legal factors that cause more harm than drugs themselves (Buchanan, 2015b; Buchanan \& Young, 2000; Denning \& Little, 2011; Levy, 2014). For 
example, Vietnamese people who use drugs often experience high levels of social dislocation as a result of their stigmatisation (Hong et al., 2011). These social and environmental disadvantages often drive people who use drugs 'underground', isolating them from normative society, and reducing their opportunities for education, outreach or employment. The environment of criminalisation and punishment also means that most Vietnamese people who use drugs are reticent about accessing health or social services for fear of exposing themselves (Edington \& Bayer, 2013). One typical example is that many people who use drugs or their peers generally do not call an ambulance (and can die of overdose) because they do not want to face prosecution and punishment for any drug use. Overall, this narrow perception of 'harm' has led Vietnam's drug policy to become punitive and abstinence-based. As this chapter demonstrates, this is based on a social and political construction rather than on any research evidence.

\section{Conclusion}

The history of drug use in Vietnam is complex and multifaceted. Throughout Vietnamese history, drug use has been negatively viewed. It was first seen as a national enemy of the state because the French were seen as using opium as a means to control and poison Vietnamese people. This perception remained until Vietnam unified in 1975 . The consequences of drug use from Vietnam's wars were difficult, as Vietnam had to deal with a great number of people who use drugs who remained dependent on drugs. Drug use was then seen as a leftover of old regimes and external contamination from the West. The tradition of viewing drug use as a poisonous force and a social evil was documented in the Vietnam 1992 Constitution. Contemporary drug use problems have further consolidated negative attitudes towards drug use in Vietnam. Authorities have begun to chart increasing numbers of people who use drugs and the development of new popular drugs. Following regional trends, opium smoking has been replaced by injecting heroin, and this has arguably triggered increasing HIV infection rates among the Vietnamese population. These conditions have further entrenched drug use as a serious social problem.

It is evident that how Vietnamese people perceive and respond to drug use is determined by wider factors. Their perceptions are influenced by political and sociocultural factors rather than evidence (Edington, 2016). The effects of market liberalisation on national identity made 
Vietnam tighten drug use controls (Wilcox, 2000), and place drug use as an immoral act that represented negative Western values rather than traditional Vietnamese values (Rydstrøm, 2006). The media and Vietnamese leaders have developed stigmatising and discriminatory representations of drug use and people who use drugs. However, these representations are counterproductive, particularly in terms of isolating people who use drugs from their communities.

For many years, the Vietnamese government and agencies like the UN have defined and classified drugs according to their legal or illegal status. Vietnamese drug classifications continue to be based on outdated UN Convention classifications from the 1960s. These classifications ignore the fact that, globally, alcohol and tobacco addiction cause far more death and health problems than any illegal drugs (Ministry of Health, 2016b). Accordingly, the Vietnamese government has tended to produce narrow and misleading perceptions of drug harms, not least that harms are caused by illicit drugs and illicit drug use. These definitions have overlooked the complexity of drug use (including the dominance of recreational use) and the role of wider social, environmental and legal factors in creating harms.

Based on the historical and contemporary perceptions of drugs, drug use, people who use drugs and harm, it is evident that negative perceptions or judgemental attitudes towards drug use and users still remain widespread among Vietnamese people and key stakeholders. Accordingly, Vietnam's drug policy is considered to be among the most punitive approaches in the world. With current negative attitudes, it is not surprising why the current responses to drug use remain deeply punitive and abstinence-focused, and harm reduction is difficult to implement (Vuong, 2016). As the next chapter further illustrates, Vietnam's drug policy still follows an approach in which punishment and prohibition are seen as key methods for addressing drug use and treating people who use drugs. 


\section{Chapter Five: Responses to Drug Use in Vietnam}

\section{Introduction}

Historic and contemporary perceptions of drugs, their use, users and harms have driven Vietnam's drug policies to be among the most punitive ${ }^{37}$ in the world. Vietnam has adopted some interventions in the name of harm reduction, like needle exchanges or methadone maintenance, but current responses to drug use remain deeply abstinence-focused. Historically, Vietnam's response to drug use focused on deterrence via harsh punishment and supply reduction measures (Hammett et al., 2008; Vuong et al., 2012). After being criticised by the USA and UNODC in the early 1990s, for being a source of illicit opium alongside Afghanistan and Laos, Vietnam emphasised the eradication of opium cultivation and was one of a handful of countries that successfully suppressed illicit opium production (Windle, 2012, 2015). However, the prevalence of drug consumption alongside the HIV epidemic and issues of addiction have led to a significant transformation ${ }^{38}$ in drug policy (Edington, 2016; Windle, 2015). Like other countries, Vietnamese policies now focus on two main domains: supply and demand reduction (abstinence-based approaches), and some so-called harm reduction interventions. Since the turn of the century, there have been many changes in the legal framework, and drug policy has arguably transformed from a punitive-based approach to one that includes a public health oriented strategy (Kincaid \& Sullivan, 2010).

Like many countries in Southeast Asia, responses to drug use in Vietnam are currently influenced by both the moral model and the disease model, which are judgemental approaches to drug use (Vuong et al., 2016). Vietnam's drug policy follows an abstinence

\footnotetext{
${ }^{37}$ Vietnam is ranked the third among countries with the harshest drug policies in the World (See https://drugabuse.com/the-20-countries-with-the-harshest-drug-laws-in-the-world/). It is not about death penalty, it is also related to compulsory treatment centres. Vietnam detained more drug users than other countries per capita rate.

${ }^{38}$ Since the early 2000s, in the wake of HIV, the perception of drug use and addiction has gradually shifted from a social evil problem to a public health issue (Heyman, 2013). Notably, the 1999 Penal Code removed the criminalisation of personal possession, which provided a basis for considering drug use as a public health matter instead of a criminal one. In 2006, the HIV Prevention Law officially acknowledged that drug use and drug addiction are public health issues.
} 
approach in which punishment and prohibition are seen as key methods for addressing drug use and addiction. While drug use is no longer criminalised, it is still cast as a social evil that needs to be eliminated within Vietnamese society. As shown previously, a wide range of antisocial evil campaigns have been implemented to prevent drug use; yet their effects are limited and just further isolate those who take illicit drugs from their families and communities. Nevertheless, in the mind-set of the Vietnamese government, reducing harm caused by drug use means the elimination of drug use through incarceration, punishment, and re-education. This fundamental misperception in the interpretation of 'harm reduction' has led to a series of misleading interventions in practice. Harm reduction is misunderstood and seen as a mechanism through which the government controls drug use and people who use drugs, including through draconian incarceration in compulsory rehabilitation centres (Windle, 2015). Such a focus on law enforcement and forced detoxification is contrary to Vietnam's commitment to decriminalise drug use, it violates people who use drugs' rights and exacerbates their problems (for example, after being recorded as a compulsory detainee, people who use drugs inevitably struggle with community integration). Therefore, although a harm reduction rhetoric has been adopted, it is reluctantly embraced in the form of needle exchange and substitute treatment in order to reduce medical problems and protect the wider society.

This chapter reviews the recent legal framework to provide a basis for understanding current Vietnamese drug policy and why an abstinence-influenced approach remains strong. The key abstinence-based approaches, supply and demand measures, are then reviewed and evaluated. The chapter highlights the negative consequences of these approaches and explains why they are still favoured in Vietnam. From here, it explores how harm reduction has developed in Vietnam, and discusses the barriers and challenges that are hindering harm reduction from moving forward. In particular, it examines the major challenges of political resistance, insufficient funding, inconsistencies in the legal framework, stigma and discrimination, as well as limited and misleading perceptions.

\section{The International Context}

Like many Asian countries, Vietnam's policy responses to illicit drugs have historically focused on demand and supply reduction measures (Devaney, Reid, \& Baldwin, 2006) but, from the 
2000s, the country employed 'harm reduction' to address drug use (Reid \& Higgs, 2011). Together with the historical and sociocultural factors, highlighted previously, these official responses have been influenced by three key external factors.

The first is that Vietnam signed and became a member of the three UN International Drug Conventions (all signed in 1997) (Vuong, 2016). Accordingly, Vietnam follows the Conventions to develop drug laws, policies and classifications, and has met regulations for drug control measures, such as punishing drug possession and implementing compulsory treatment or rehabilitation. The country has even adopted punitive measures that 'are beyond Treaty requirements, like detaining people who use drugs in coercive treatment centres for two to five years without due process' (Vuong et al., 2016, p. 3).

The second external factor is that Vietnam joined the ASEAN ${ }^{39}$ Senior Officials on Drugs (ASOD) in 1995 (Ahmed et al., 2014). In 2000, ASOD set a target of creating a 'drug-free ASEAN region' 40 by 2015 , a plan that was strongly supported by the United Nations Office on Drugs and Crime (UNODC) (Vuong et al., 2016). Vietnam implemented a number of measures to reach this drug-free target, such as imposing tougher national drug laws, strengthening the cooperation between ASEAN agencies involved with narcotics prevention, and joining ASEAN Police (ASEANPOL) (Vuong et al., 2016). These measures, that reflect the moral model of addiction, have reinforced punitive drug laws.

The third important factor is the changing relationship between Vietnam and the USA. In 1994, the USA lifted the embargo against Vietnam (imposed from 1975), and normalised bilateral relations. In 2003, Vietnam and the USA signed the 'Counter Narcotics Letter of Agreement', which developed closer cooperation in narcotics prevention (Vuong et al., 2016). Also, as HIV infection emerged as a serious problem in Vietnam, US-sponsored international

\footnotetext{
${ }^{39}$ ASEAN, Association of Southeast Asian Nations, is a geo-political and economic organisation of ten countries located in Southeast Asia. These Asian countries have cooperated in many fields, ranging from socio-cultural and economic to political and security issues.

${ }^{40}$ Inspired by the United Nation's Goal 'A Drug Free World', ASEAN has tried to achieve th is goal. Illicit drugs, as argued previously, are seen as social problems and a threat to the prosperity of ASEAN region. However, as with the global drug wars, the region has failed to achieve its goal as the illicit drug market continues to expand, the consumption of illicit drugs and the number of people who use drugs remains consistently high (Luong, 2017).
} 
organisations, such as the World Health Organization (WHO), the Joint United Nations Program on HIV/AIDS (UNAIDS), the World Bank (WB), and the United Nations Office on Drugs and Crime (UNODC), have become indispensable donors and partners for drug prevention and treatment measures, even though their financial support has declined since Vietnam became a middle-income country in 2010 (Edington \& Bayer, 2013). These organisations and the USA have widely endorsed addiction as a brain disease model of addiction that tries to reduce drug use and seeks abstinence, so it is understandable why Vietnam's drug policy has been widely influenced by abstinence-based ideologies.

In summary, the Vietnamese government has emphasised tough law enforcement and punitive-based approaches towards drug use problems due to the three above external factors. Based on the UN Conventions and the goal of a 'drug-free ASEAN region', Vietnam's target is to be a drug-free country. This ideology (together with that of drug use as a 'social evil') has influenced every aspect of Vietnam's government responses to drug use. As a result, although Vietnam has implemented some measures in the name of harm reduction, the remit of abstinence has remained widespread and has guided legal frameworks.

\section{Legal Framework}

The nature of Vietnam's drug policy can be clearly identified through legal developments. With the rise of HIV infections and illicit drug use rates in the early 1990s, Vietnamese government officials established severe measures against users, drug dealers and traffickers (Ahmed et al., 2014; Hammett et al., 2008). The primary philosophy that underpins Vietnamese drug policy is demonstrated in Article 61 of the 1992 Constitution, which stipulates that:

It is strictly forbidden to produce, transport, trade in, possess and use opium and other narcotics. The State provides for compulsory treatment of drug addiction and certain dangerous social diseases (i.e., associated illicit drug use and sex work) (Vuong, 2016, p. 2).

The 1999 Criminal Code established harsher punishments like life imprisonment and even the death penalty for drug trafficking. Those using any types of illicit drugs would be sentenced to between three months and two years of imprisonment, depending on how many times 
they were detected using illicit drugs (Kincaid \& Sullivan, 2010). In 2000, the National Assembly passed the law on 'Narcotic Drugs Prevention and Suppression'. This law reinforced drug use as a social problem and promoted the detention, treatment and rehabilitation of those engaged in social evil (Ahmed et al., 2014). Thus, in 2003, the Vietnam National Assembly adopted 'Resolution No.16' to enhance treatment and detoxification services; as a result, compulsory treatment in detention centres, in the name of rehabilitation and occupational training, was extended to up to six years (WHO, 2009).

In 2006, there was a marked shift in drug policy when the law on 'HIV/AIDS Prevention and Control' was passed by the National Assembly. This law paved the way for altering public perceptions of drug use problems. Under this law, drug use was seen as a public health issue and users were generally regarded as patients. The law facilitated the adoption of harm reduction because it further encouraged the free distribution of sterile needles and syringes, the implementation of substitute treatments and other harm reduction interventions (Vuong et al., 2012). As a consequence, significant resources were allocated to HIV testing, counselling, and antiretroviral (ARV) treatment (WHO, 2009).

Importantly, in 2009, the New Criminal Code abolished Article 199 of the 1999 Criminal Code, which regulated drug use as criminal behaviour. People who use drugs were now characterised as patients, not criminals. Influenced by the brain disease/medical model, this law engaged more people who use drugs in addiction treatment and harm reduction services. Ironically, although Vietnam decriminalised all illicit drug use in 2009, people who use drugs still receive 'administrative sanctions' ${ }^{41}$ under the 2012 Administrative Violation Law (Kincaid \& Sullivan, 2010). For example, as discussed further below, people who use drugs can still be fined and sent to compulsory treatment centres if they test positive for illicit drugs. Vietnam has contradictory legal frameworks, as it continues to expand medical interventions in the name of harm reduction.

Since 2011, new policies and strategies on HIV prevention, harm reduction, drug treatment and drug control have been enacted and amended, facilitating a multi-sectoral collaboration

\footnotetext{
${ }^{41}$ The 2012 Administrative Violation Law categorises drug use as an administrative violation, and people who use drugs can be subjected to a warning, fined or face administrative detention for up to two years.
} 
between three key government agencies, namely the Ministry of Public Security (MPS) ${ }^{42}$ the Ministry of Labour, War Invalids and Social Affairs (MOLISA) ${ }^{43}$ and the Ministry of Health $(\mathrm{MOH})^{44}$ (Ahmed et al., 2014). In June 2011, the Vietnamese Prime Minister, Nguyen Tan Dung, passed the National Strategy on Drug Control with a 2020-2030 Vision (Vuong et al., 2012). This strategy emphasised the role of HIV prevention and harm reduction (Windle, 2015). From 2012, the country committed to continue to scale up the distribution of sterile needles and syringes, substitute treatment interventions, and implement harm reduction interventions such as community-based treatment and after-care treatment models (Ahmed et al., 2014). In June 2012, the National Assembly of Vietnam voted to close traditional rehabilitation and adopted the Drug Rehabilitation Renovation Plan 2013-2020, ${ }^{45}$ which recognised drug addiction as a medical issue (Edington \& Bayer, 2013). This plan aims to promote the expansion of community-based treatment and the gradual closure of compulsory treatment, which are to facilitate harm reduction in the country. Yet the plan generally is only valid on paper, and barriers for 'authentic' harm reduction remain almost unchanged (discussed further later). Figure 5.1 (below) illustrates how Vietnam's drug policies have changed since 1992.

\footnotetext{
${ }^{42}$ The MPS formulates policies, plans, and strategies for fighting against drugs crimes. In terms of addiction treatment, the MPS helps MOLISA to ensure the security of compulsory treatment centres. It also cooperates with MOLISA to supervise people who use drugs after they are released from the centres, in community based treatment.

${ }^{43}$ MOLISA organises and manages drug treatment at centres and communities over the whole country. The Ministry also collaborates with MPS by sending people who use drugs to local police, and it collaborates with the Ministry of Health in terms of addiction treatment.

${ }^{44}$ The $\mathrm{MOH}$ regulates lists of addictive drugs, psychotropic drugs and precursors that can be used to produce synthetic drugs. The Ministry is empowered to provide medicines for detoxification and to prescribe addiction therapies. The $\mathrm{MOH}$ has been charged with running harm-reduction interventions.

${ }^{45}$ This National Plan targets the reduction of the 06 system, and prioritises community and family based treatment (MOLISA, 2015).
} 


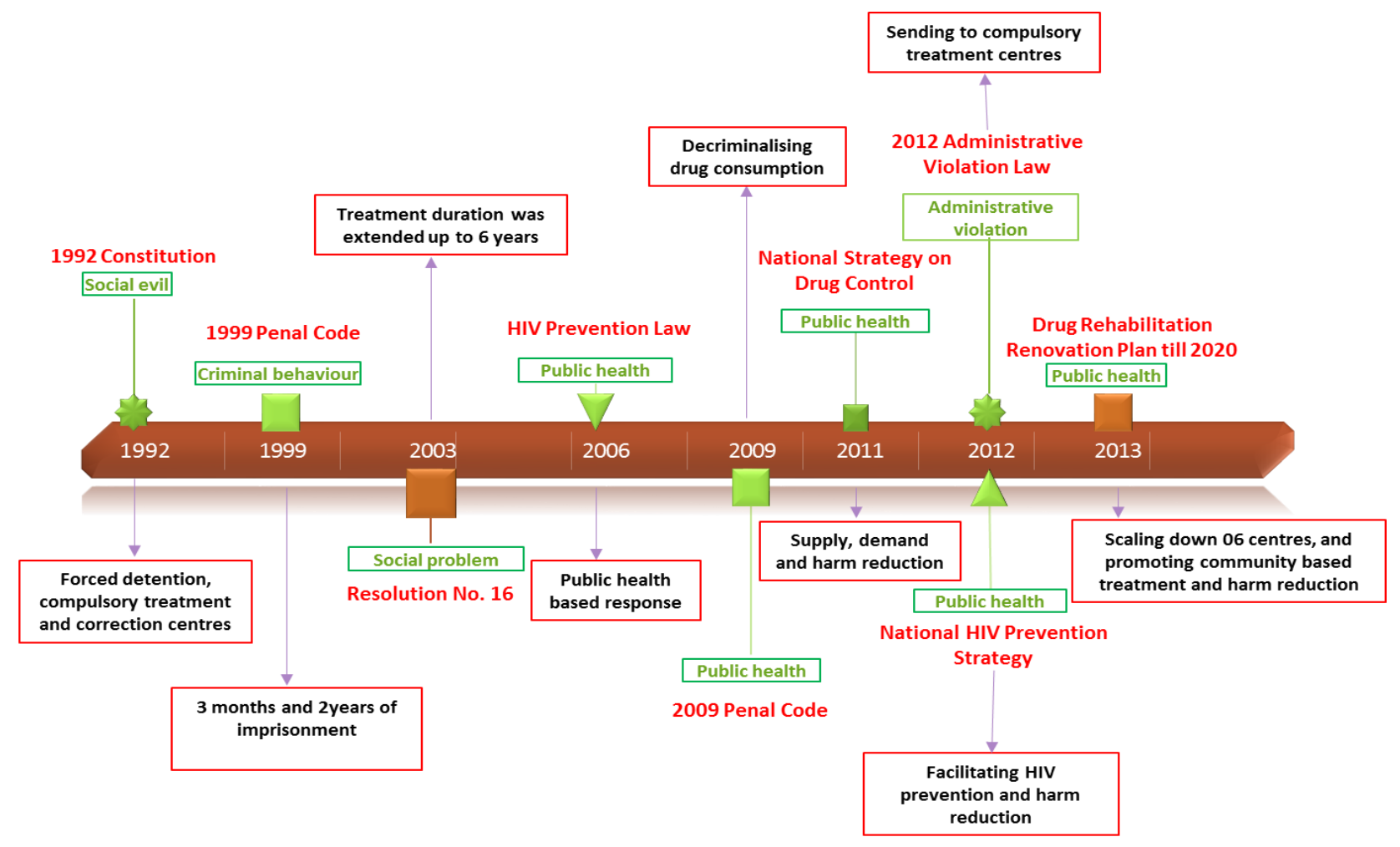

This figure shows the development of Vietnam's drug policy from 1992. It summarises the key milestones on legal frameworks, the most prioritised drug policies and how the government perceives drug use on paper. Specifically, the red highlighted words illustrate legal and policy frameworks, the black highlighted words represent the main response to drug use, while the green ones show how the Vietnam government defines drug use. Although from legal perspectives, drug use has been regarded as a public health issue since 2006 , in reality, the issues of social evil, criminality and public health have all existed and converged at the same time. These overlaps have influenced Vietnam's drug policy and produced some confusing results - most noticeably that, as this thesis demonstrates, Vietnam has attempted to implement 'pseudo' harm reduction within an abstinence-based foundation and mind-set.

In summary, the landscape of Vietnam's drug policy has changed from the 1990s. The legal framework has moved slowly from a stringent punitive approach toward strategies that include public health. The 2006 Law on AIDS/HIV Prevention and Control shifted policies toward harm reduction (Windle, 2015). Vietnam has since made commitments to scale down

${ }^{46}$ Devised by the author. 
the system of drug detention centres to community-based treatment approaches and harm reduction on paper, but abstinence-based strategies (including supply and demand reduction strategies) remain prominent in practice (Edington \& Bayer, 2013). When drug consumption became widespread, Vietnam started suppressing opium production, strengthening activities against drug crimes, and deploying more anti-drug education campaigns and addiction treatment programmes to curb the expansion of drugs and drug consumption. The next sections further illustrate why supply and demand reduction remain dominant in Vietnam despite their limited effectiveness in practice.

\section{Supply Reduction}

Supply reduction is considered an essential component of drug control policy in Vietnam (Duc, 2012). By imposing stricter regulation and law enforcement, this approach is believed to address the root causes of drug use problems. Arguably, demand reduction approaches cannot be successful unless drug availability is restricted ( $\mathrm{Vu}, 2000)$, and the Vietnamese government has subsequently put plenty of effort into supply reduction interventions including tightening up drug laws, suppressing illicit drug production, and preventing and combating drug crimes.

Vietnam is one of only a handful of states that have successfully suppressed illicit opium production, achieving a decline of $98 \%$ in production from the 1980 s to the 2000 s (Windle, 2012 ) - in 1985-1986 the opium poppy crop was 19,000 hectares, reduced to 300 hectares by 2001-2002 (mainly in the mountainous Nghe An, Son La and Lai Chau provinces) (MPS, 2015). Vietnam is no longer seen as a major source of opium production. Figure 5.2 below shows the number of opium hectares that were cultivated over the last 25 years. 


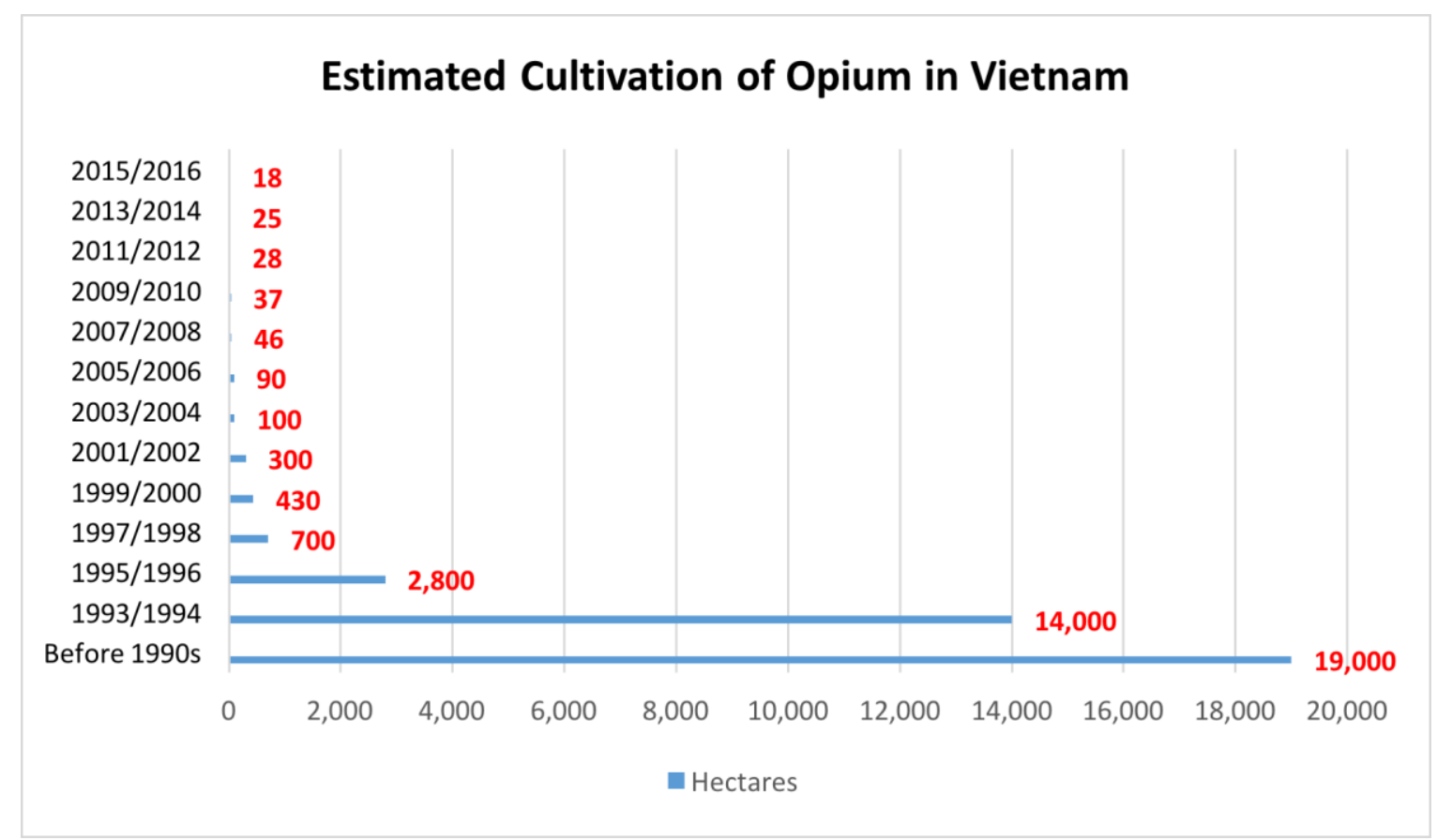

Although Vietnam succeeded in reducing opium cultivation, many people living in the mountainous north-west areas have continued to engage in drug trafficking and drug smuggling. For example, according to the report of UNODC (2010), 48\% of heroin trafficking and $92 \%$ of opium trafficking into Vietnam were related to people who live along the northwest border of Vietnam. The eradication just meant that opium was brought in from other countries, and it has not stopped supply. This shows the futility of the supply reduction approach - the remarkable decline in opium production has had relatively little impact on Vietnam's illicit drug consumption, as drug use and the number of people who use drugs have increased year by year (UNODC, 2010).

In addition to suppressing illicit drug production (mainly opium), the Vietnamese government has established and engaged many agencies (such as police, customs, border guards, and coast guards) to enhance drug law enforcement for the purpose of preventing illicit drug use and supply. Yet it was reported by Vietnam's UNODC $(2015)^{47}$ that drug crimes like drug

\footnotetext{
${ }^{47}$ From 1980 to 1990, there were around 1,700 reported criminal cases related to illicit drugs, while in the next ten years (1991-2000) over 43,000 cases were recorded, an increase of over 25 times. Later, the number of reported criminal cases related to illicit drugs continued to increase and after 2011, on average there were around 20,000 cases per year (ASEAN InterParliamentary Assembly, 2015; UNODC, 2010).
} 
trafficking and smuggling have significantly risen since the 1990s, due to the increases in policing and law enforcement activities, which means that higher levels of law enforcement have ensured detection of higher numbers of drug crimes by police and other relevant agencies (Windle, 2012). Compared to Vietnam's targets for illicit drug control, the country has, by and large, failed to achieve its goals and the ASEAN region goal, which is to create a drug-free society. These failures are not only confirmed in Vietnamese government documents, but in many relevant studies (Human Rights Watch, 2011, 2012; Vuong et al., 2012; Windle, 2015). As Luong (2017, p. 215) indicates:

Except for policy-makers and leaders, who believe "the commitment of achieving a drug-free ASEAN by 2015 is still valid" ${ }^{48}$, most scholars believe that the goals of a drugfree world or drug-free Asia will almost certainly never be achieved.

Many Vietnamese policy-makers have realised that the punitive-based law enforcement interventions (supply reduction activities) did not address and eliminate drug use problems in the country. Instead, these interventions have often fuelled stigma and discrimination towards people who use drugs rather than giving them necessary assistance (Edington, 2016). By and large, the resourcing (funds, people) devoted to the activities of policing, regulation, or border controls has not been successful in dealing with the 'social problem' of drug use in Vietnam because, as noted above, drug use and the number of people who use drugs have increased. Although supply reduction is still regarded as the main solution for drug use, Vietnam's government has increasingly paid more attention to demand reduction activities, like education campaigns and treatment. Yet these measures have also been limited and controversial (Edington, 2016).

\section{Demand Reduction}

While much effort has been made to reduce the supply source, the Vietnamese Government has recently promoted demand reduction interventions that include education and media

\footnotetext{
${ }^{48}$ As noted previously, many policy-makers and leaders and the media frequently target that they will create a clean community or a drug free community (Muncie, 2010). Many leaders also use the drug-free society campaign as means of receiving public attention and creating opportunities for career promotion. These common perceptions are linked to 'Populism', which was discussed in Chapter Two.
} 
campaigns against drugs and drug use and, importantly, the promotion of addiction treatment (Vuong et al., 2016). From the 2000s, in particular, the government has launched large-scale propaganda campaigns to prevent social evils, and developed a system of compulsory treatment (Edington, 2016). In many respects, there is a disconnection between official commitments and actual responses to drugs and people who use drugs in Vietnam.

\section{Anti-Drug Education Campaigns}

Anti-drug education campaigns aim to raise public awareness of the dangers of drugs and drug use. They essentially aspire to drive people away from drugs (MOLISA, 2015). A popular social movement called 'Toan dan tham gia phong chong kiem soat ma tuy' (which can be translated as 'engaging public participation in drug control activities') has been conducted since the 1990s (Vu, 2007). Vietnamese authorities have collaborated with schools, communities and both public and private organisations in drug use prevention programmes. The state has also launched many campaigns against drug use from the 1990s, for the purpose of eliminating 'poisonous forces' from Vietnamese society (Edington, 2016, p. 6). As Images 5.1 and 5.2 demonstrate, the campaigns tend to use negative representations of drug use, referring to illicit drugs alongside pictures of skulls and bones, and the words 'The Dead Chorus' or 'Drugs kill your Family' to express the consequences of drug use (Nguyen \& Scannapieco, 2008, p. 21). 


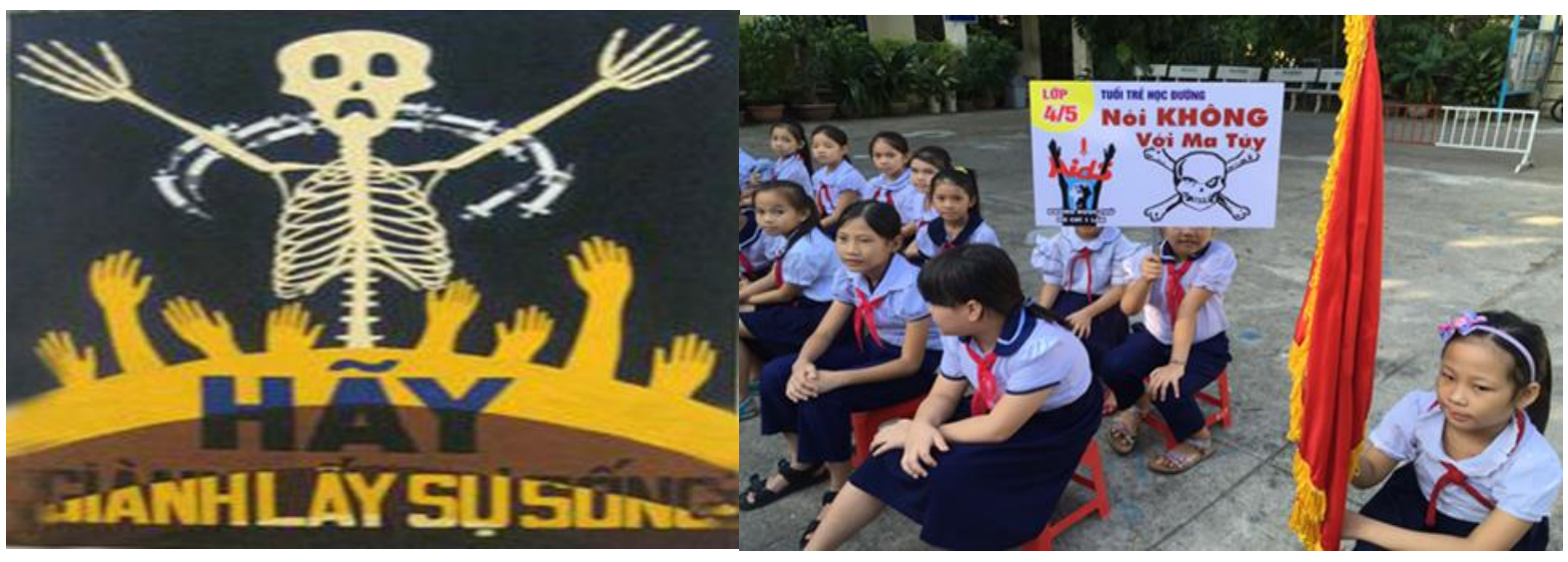

Image 5.1 (Left): Saving human's lives from narcotics (illicit drugs) (The National Committee on AIDS Drugs and Prostitution Prevention and Control 2011)

Image 5.2 (Right): Students say no to narcotics (The National Committee on AIDS Drugs and Prostitution Prevention and Control 2011)

Although the Vietnamese government has made great efforts to expand the anti-drug campaign, drug use appears to have extended from rural to urban users and to have involved younger users, over recent years (Nguyen \& Scannapieco, 2008). There has been no corresponding reduction in the number of people who use drugs. The images cement the fact that drug use is seen as a criminal issue, not one of public health, so official approaches have generally promoted stigma and discrimination towards people who use drugs. Negative caricatures and stereotypes encourage people to shun and even abandon people who use drugs (Nguyen \& Scannapieco, 2008). These failures are replicated globally - for example, studies by Chermak (1997), Erceg-Hurn (2008), and Taylor (2008) show that government-led media campaigns have had no significant impact on drug use trends, and the dangers of identified drugs were over exaggerated. Ironically, such campaigns have increased social stigma and isolation, and promote judgemental attitudes towards people who use drugs, and cause further criminality among this group.

\section{Drug Treatment}

As noted previously, the Vietnamese government views drug use and addiction as a moral, individual problem, or as a result of biological or psychological defects. Accordingly, solutions or interventions against addiction in Vietnam are generally placed at the level of the person by repairing biological or psychological defects, rather than social and environmental issues. 
Vietnam has long conducted coercive treatment for drug addiction. In 1989, the Vietnamese Government Assembly regulated that 'all clinic centres have to implement compulsory treatment measures for drug addicts' (MOLISA, 2015). Treatment measures were then focused on the incarceration of people who use drugs in compulsory treatment centres (06 centres) that would attend to this 'dangerous social disease' (art. 61, 1992 Constitution) (Vuong et al., 2016). The compulsory drug treatment measure was further strengthened in 1993, as the government issued Resolution No. 06 / CP, which specified that:

Regarding addicts, engaging in drug addiction treatment centres is compulsory ... depending on a case by case, they can participate in drug addiction treatment in staterun drug rehabilitation centres or private centres (MOLISA, 2015, p. 48).

Those seen to be heavily addicted to illicit drugs, who fail to accomplish 'community and family-based treatment ${ }^{\prime 49}$ or who relapse can be forced into compulsory treatment (MOLISA, 2015). Compulsory treatment programmes in Vietnam are believed to mirror the principle of drug treatment of the National Institute on Drug Abuse (NIDA) of the Department of Health and Human Services USA (USDHHS), UNODC and WHO, stating that the treatment of addiction does not need to be voluntarily undertaken to be effective (MOLISA, 2015). This narrative has encouraged many countries to expand the system of compulsory treatment centres, Vietnam included. Although these centres have been criticised for being ineffective, costly and violating human rights (Kamarulzaman \& McBrayer, 2015), they remain widely used. In 2017, MOLISA managed 123 compulsory treatment centres (56 centres in 2000 and 109 centres in 2009) and over 17,000 people who use drugs were treated in these centres (Vuong et al., 2017). Apparently, the commitment to decrease these centres is just lip service and it is not happening in reality.

${ }^{49}$ The communal people's committees are responsible for implementing treatment work at the family and community level. They are supported by local leaders, health workers, social workers, police, and members of women's and youth associations. In addition to detoxification, psychological therapies and other therapies will be offered. The communal People's Committees are also responsible for organising cultural activities, arts, sports and other social activities, as well as providing vocational training, finding a job, providing loans, and helping users to reintegrate into the community (MOLISA, 2015). Yet these supports generally exist only on paper, as the stigmatisation of and discrimination against people who use drugs in local communities are widespread. 
The government provides several arguments for the existence of 06 centres (MOLISA, 2015). Firstly, the centres are argued to be a humanitarian response because they help people who use drugs to stay away from drugs, overcome their problems, and become aware of the harmful effects of drug dependence. Secondly, counselling, and advanced training in terms of moral education, addiction prevention and HIV prevention are provided to prepare and equip people who use drugs with necessary skills before they re-enter the community. Lastly, the government thinks that sending people who use drugs to the centres contributes to reducing the crime rate and ensuring social order and safety, and importantly protects the wider community from this 'high risk' group (Nguyen, 2002). These perspectives prioritise punitive addiction-based responses to people who use drugs that is very much at odds with the purported harm reduction stance (Edington \& Bayer, 2013).

The general perception among police is that detaining people who use drugs in the centres can reduce threats to the wider community (Windle, 2015). Further, as Khuat et al. (2012) argue, police have also been tasked to meet arrest quotas to keep the centres at capacity. Importantly, profits made through commercial contracts signed between the centres and other private companies are also seen as an incentive or economic motivation for retaining the centres (Vuong et al., 2012). The majority of detainees have to work without contracts or receiving wages for their labour, meaning that they provide free labour for the economic benefit of the centres (Edington \& Bayer, 2013). Labour therapy, which includes job and vocational training, is mainly justified by the Vietnamese government as a stepping stone for community integration; yet it is widely reported that training in the centres is not applicable to the labour market (Human Rights Watch, 2012). The majority of former detainees had also reported that they have encountered a high level of stigma and discrimination because they are labelled as 06 detainees and judged to have poor moral character (Tomori et al., 2014), making it difficult for people who use drugs to find a job on release (Windle, 2015). As a consequence, the majority of those released continue drug use as a way to overcome the boredom and despair of stigma and unemployment.

A study by Edington and Bayer (2013) showed that these centres contain strong seeds of both financial and political capital, which are associated with discourses of populism discussed in Chapter Two. For example, one research participant from their study explained: 
Nguyen Minh Triet, the President of Vietnam, was a champion in Ho Chi Minh City for this centre approach and built his career on it. The deputy mayor of Ho Chi Minh City is now doing the same thing. People are seeing that in taking this approach, you are able to demonstrate popularity with local residents, you're getting people who use drugs off the street, you're able to get political favour, and you're able to provide economic benefit ... or a whole lot of labour factories. So there are a lot of advantages and very little disadvantages in the way that the system is run (Edington \& Bayer, 2013, p. 87).

Similarly, as Edington and Bayer (2013, p. 88) reveal:

Those running the centres are not health officials and, in fact, have a different kind of cost benefit analysis in mind that includes monies made by contracting detainees in the service of private companies.

In fact, the 06 centres have been heavily criticised as being harmful, ineffective and counterproductive for people who use drugs (Human Rights Watch, 2011; UNAIDS, 2016). Run by MOLISA rather than $\mathrm{MOH}$, and operated by non-health officials, their ability to provide medical or psychological interventions is minimal (Edington \& Bayer, 2013). Contrary to the list of commitments presented to parents/people who use drugs before admission, detention (not treatment) is prioritised in the centres. If treatment is offered, it is generally based on health officials' compulsory plans, instead of having a consensus between patients and doctors (Vuong et al., 2016). In other words, as Windle (2015) and UNAIDS (2012) state, the treatment is not tailored to individual needs or circumstances.

As a result, estimated relapse rates are extremely high, at around 90\% (Human Rights Watch, 2011; Kamarulzaman \& McBrayer, 2015). These contradict official figures (of relapse rates of 50-60 \%) presented in the 2011 National Strategy on Drug Control (Human Rights Watch, 2012). In addition, the likelihood of transmission of HIV is higher in the centres than in communities because, contrary to a harm reduction philosophy, detainees cannot access sterile needles and syringes (Vuong et al., 2012; Windle, 2015). It was estimated that the HIV prevalence among drug detention centre detainees ranged from 30\% to 60\% (Human Rights Watch, 2012). Also, like many East and Southeast Asian countries, in Vietnam centre-based 
compulsory treatment is often applied to those who use illicit drugs, even though they have not engaged in criminal behaviour beyond drug-taking (Vuong et al., 2017). Centre detainees also include recreational users, who do not need treatment, because (as noted previously) Vietnam generally does not distinguish between different types of people who use drugs. In the centres, physical beating, punishment and even torture have also been reported by detainees, especially when they refused to work or did not work fast enough to keep pace with other detainees (Amon, Pearshouse, Cohen, \& Schleifer, 2013). Obviously, although the government commits to treat people who use drugs as patients on paper, they treat detainees as offenders or prisoners in practice (UNAIDS, 2016).

The Vietnamese government has exhibited a diplomatic attitude in response to human rights criticisms (Vuong et al., 2017), declaring its intention to reform and reduce the number of 06 centres (Windle, 2015). One of the biggest challenges for scaling down and closing the centres is that, as Vuong et al. (2017, p. 11) explain:

They [Vietnamese government] also expressed their confusion about why compulsory treatment in Vietnam was criticized when it was seen as acceptable in developed countries and when UNODC in their endorsed principles of treatment claimed that 'compulsory treatment could be effective'.

In fact, as Edington (2016) argues, the system of the 06 centres is considered part of 'a continuum of policy designed to reduce the harms of drug use to society' and they are 'too strongly entrenched to be abolished' (pp. 11, 12), so expecting immediate closures from the Vietnamese government is overly optimistic (as noted above, there were still 123 centres in 2017).

In summary, it is evident that current repressive Vietnamese law enforcement interventions and the compulsory detention of people who use drugs have been ineffective, resulting in the high level of stigma and discrimination towards people who use drugs (Edington, 2016; International Network of People Who Use Drugs, 2014; UNAIDS, 2016; WHO, 2009). Similar to global responses to drug use, Vietnam has implemented controls over specific social groups rather than finding rational and effective evidence-based solutions for drug use problems. As argued earlier, Vietnamese responses to drug use have failed and resulted in many 
disadvantages to people who use drugs. Punishing people who use drugs and detaining them in the 06 centres cannot be proper treatment for their drug use, instead these measures further drive people who use drugs to desperation and social dislocation - and exacerbate the reasons for taking drugs (Edington \& Bayer, 2013). People who use drugs still relapse, are unable to find employment and struggle to reintegrate into society. As Seddon (2007) and Stevens (2012) argue, coercive treatment is not effective, violates human rights and relapse rates are high. Importantly, many people who use drugs also report high levels of blood-borne diseases because treatment services are limited. In such circumstances, forcing people who use drugs into treatment becomes counterproductive. It is evident that 06 centres have been the antithesis of an authentic harm reduction approach.

Therefore, transforming current punitive-based approaches and fruitless abstinence-based interventions is necessary. As Edington (2016) argues, current approaches to drug use in Vietnam have resulted in high levels of stigma and discrimination which serve as major disincentives for people who use drugs to access treatment or other services. The harms of problematic drug use are not reduced from dominant official responses. However, the harm reduction approach that Vietnam also articulates should decouple the discourse of social evil from HIV and drug use. As defined in Chapter Three, authentic harm reduction is an approach that seeks to reduce the negative consequences of drug use, for everyone (Ritter \& Cameron, 2006; Ritter et al., 2011). Harm reduction strategies can make drug use safer and provide the necessary support to people who use drugs, their families and communities.

\section{Harm Reduction in Vietnam}

The failings of supply and demand reduction measures, and the escalation of the HIV/AIDS epidemic, have contributed to the adoption of some medical interventions in the name of harm reduction in Vietnam. In addition, Vietnam has tried to change the public mind-sets from seeing drug use as a social evil to a public health matter, but this has happened slowly in practice. In fact, a combination of the two approaches is more obvious. This explains why so-called harm reduction in Vietnam has only focused on public health-oriented responses towards problematic drug users. This section explores how harm reduction interventions have developed, what interventions have been implemented, and their outcomes in the country. The section also examines the barriers and challenges to consolidating harm 
reduction practices in Vietnam. Importantly, it demonstrates why Vietnam has conducted pseudo harm reduction.

\section{A Chronology of Harm Reduction in Vietnam}

In response to the HIV/AIDS epidemic, some harm reduction initiatives (mainly needle exchanges) were introduced to Vietnam during the 1990s. The first methadone maintenance programme began in Ho Chi Minh City in 1996, but was discontinued for financial reasons in 2000 (Edington \& Bayer, 2013; Reid \& Higgs, 2011). However, in 2002, with financial support from international donors, a small-scale methadone programme was re-launched (Edington \& Bayer, 2013). Following the 2004 'National Strategy for Prevention and Control of HIV/AIDS', which listed harm reduction as one of the main goals of a national HIV response (Edington \& Bayer, 2013), harm reduction interventions like methadone or needle exchange programmes have expanded.

In 2006, harm reduction was officially adopted with the 'Law on HIV/AIDS Prevention and Control' (HIV Law) (Heyman, 2013). Defining harm reduction (Article 2, clause 15) as 'methods that encourage the use of condoms, sterile needles and syringes as well as the treatment of dependent people who use drugs through the provision of substitute substances', the Law established a narrowed public health approach to harm reduction. This has meant that interventions like HIV testing, care or treatment have been promoted rather than the overall philosophy of reducing harms from drug use per se (Ritter \& Cameron, 2006).

Since 2006, a larger source of income has been allocated for HIV testing, counselling, and antiretroviral (ARV) medications, so the number of people who use drugs engaged in treatment has increased rapidly (Khuat et al., 2012). In 2007, the Vietnam government issued Decree 108 to carry out a detailed plan of action emphasising the important role of the Ministry of Health in HIV prevention and harm reduction (Ahmed et al., 2014). Since then, Vietnam has continued to show a strong leadership and political commitment for the development of harm reduction interventions. Notably, in June 2012, the National Assembly of Vietnam voted to emphasise voluntary and community-based treatment (Edington \& Bayer, 2013). 
In 2013, the Vietnam government promised that 80 of the 107 compulsory treatment centres (in 2012) would be reformed to be voluntary and friendly detoxification centres and that harm reduction (mainly methadone services) would soon be available (Kamarulzaman \& McBrayer, 2015). Although Vietnam promised to scale down the 06 system, compulsory treatment centres were still expanded, rising from 107 centres in 2012 to 123 centres in 2015 (MOLISA, 2015). This contradicts the government's 2013 commitment. This irony raises the question: how can Vietnam, a country that accepts harm reduction and receives extensive financial funding from international donors for its implementation until now, still support the existence and even the expansion of the 06 centres? Obviously, Vietnam has a strong commitment to abstinence (a punitive approach), even though it also implements some forms of pseudo harm reduction. Therefore, thinking that Vietnam's drug policy has transformed from punitive control measures to a more rights-based harm reduction approach can be contested. Actually, Vietnam has only developed narrow forms of harm reduction in practice.

\section{Harm Reduction Interventions}

Vietnam has attempted to guide harm reduction interventions by scientific evidence (Kincaid \& Sullivan, 2010). Yet harm reduction remains firmly rooted in a narrow public health perspective. The country has widely implemented harm reduction programmes related to addressing HIV infection and drug addiction, including (i) peer education and outreach, (ii) needle and syringe distribution, and (iii) substitution treatment. However, other common harm reduction interventions, such as opening drug consumption rooms or providing people who use drugs with clean injectable drugs, or adopting the actual philosophy behind authentic harm reduction have not developed.

\section{Peer Education and Outreach Programmes}

Peer education and outreach services are considered an important part of the HIV prevention strategy in Vietnam (VAAC, 2011). The aims of these programmes are to provide people who use drugs with HIV prevention education, risk reduction methods related to drug use, 
counselling or testing information. The public health sectors work with 'peer educators', 50 support groups, local police and some international organisations to provide harm reduction information and services (Vuong et al., 2012). Those participating in peer education and outreach are trained in health promotion, HIV prevention and harm reduction, and also have responsibilities for distributing clean injecting equipment and collecting used syringes. Peer educators are essential because they not only bring prevention messages to drug user populations, but also connect those users to necessary care and treatment services. Furthermore, overdose prevention information and training (e.g. using naloxone ${ }^{51}$ ) have been recently introduced to people who use drugs when they engage in peer education programmes (Hayes-Larson et al., 2013). The exact numbers of peer education and outreach programmes in Vietnam are not available but, according to Ministry of Health (2018b), most HIV counselling centres, needle distribution units and methadone treatment clinics in Vietnam integrate peer education and outreach programmes into their activities. In general, these programmes are effective in reducing HIV infection among people who use drugs and engaging more users in health services, so the programmes receive strong support from the Vietnamese government and international organisations.

\section{Needle and Syringe Distribution Programmes (NSDPs)}

The 2006 HIV Prevention and Control law paved the way for the expansion of needles and syringe programmes in Vietnam. In 2005, only 21 cities and provinces had implemented NSDPs. This grew to 42 in 2007, and to 60 cities and provinces in 2009 (VAAC, 2011). The proportion of people who inject drugs who used sterile injecting equipment had increased, rising from 89\% in 2006 to 94\% in 2009 (Ministry of Health, 2016b). These programmes relied heavily on donor-funding from the UN and other international organisations, but funding has been gradually reduced since Vietnam became a middle-income country in 2010 (Vuong et al., 2012). In 2017, NSDPs operated in 53/63 cities and provinces (Ministry of Health, 2018b), yet people who use drugs are now able to buy needles and syringes in pharmacy stores to

\footnotetext{
${ }^{50}$ Peer educators are people who use drugs who joined the programmes and were trained in basic HIV prevention and harm reduction. They assist other service providers in helping people who use drugs.

${ }^{51}$ Drug overdose medicine that reverses the effects of opiate overdose.
} 
practise safe injection. The significantly decreased prevalence of HIV infection among IDUs, dropping from about 65\% in the 2000s to 9\% in 2015 (Ministry of Health, 2016b), has demonstrated the effectiveness of NSDPs in Vietnam.

\section{Substitution Treatment Programmes}

Although harm reduction was officially accepted in 2006, small pilot methadone maintenance programmes were conducted to treat opioid addiction from 1997 to 2002 (Vuong et al., 2012). The pilot results were positive and this led to a nationwide expansion of methadone programmes from 2006. In 2008, the government enabled the establishment of methadone maintenance treatment (MMT) in Ho Chi Minh City and Hai Phong City. By September 2009, methadone treatment was available in nine provinces and cities, with 20 centres treating 4,904 patients (VAAC, 2011). Noticeably, in June 2011, MOLISA, which argued against harm reduction, adopted methadone treatment in their addiction treatment centre system. This new model is a 'co-pay programme' meaning that both patients and government share expenses to buy methadone $-30 \%$ for the former and the rest for the latter (Vuong et al., 2012). This harm reduction programme has subsequently scaled up, providing services to people who use drugs in Vietnam (Ministry of Health, 2016a) (see Figure 5.3).

Figure 5.3 The number of methadone patients in Vietnam (2008-2017)

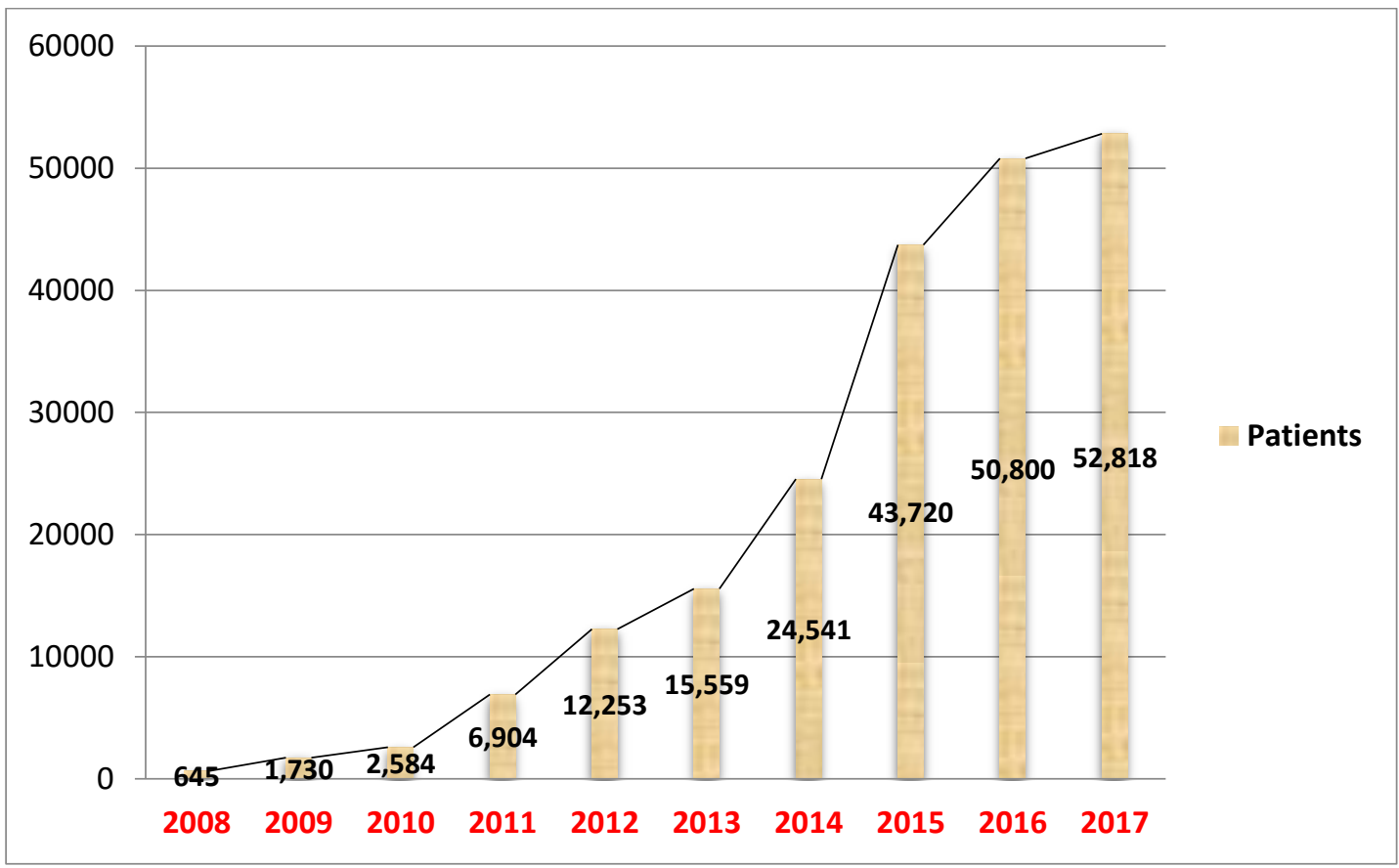


Methadone clinics have been mainly managed by $\mathrm{MOH}$. In general, methadone treatment has been considered effective in reducing HIV risk, and in improving the social and health status of people who use drugs (Le et al., 2013; Ministry of Health, 2016a; Tran, Ohinmaa, Duong, Do, Nguyen, Mills, et al., 2012; VAAC, 2011). In 2014, the Vietnamese government approved the implementation of pilot buprenorphine and Suboxone treatment programmes in Ho Chi Minh City and Ha Noi City, and in 2015 these programmes were scaled up to Thanh Hoa and Bac Giang Province (Ministry of Health, 2016b). Recently, overdose prevention programmes (using naloxone) have been integrated into methadone clinics across the whole country (Ministry of Health, 2016b). Nevertheless, unlike authentic harm reduction approaches, people who use drugs usually have to follow doctors' or nurses' instructions rather than make informed decisions for their treatment. Further, these methadone programmes operate with a high threshold approach, meaning that people who use drugs must meet many 'requirements' 52 to receive any benefits. These issues really demonstrate the contradictions in approaches in Vietnam, and that the country's approaches have not yet adhered to authentic harm reduction.

In summary, following the official endorsement of some harm reduction interventions in 2006, Vietnam has made significant positive progress. Nonetheless, it is evident that harm reduction interventions are medicalised, reflecting a narrow pseudo perspective of harm reduction. The next section analyses some of the ongoing challenges in the area and demonstrates that while Vietnam has a legal framework for harm reduction, current approaches remain linked to abstinence principles.

\section{Barriers/Challenges}

Significant challenges and barriers to effective harm reduction remain. This section considers the issues that constrain the support and expansion of harm reduction interventions, reflecting upon: (i) insufficient financial resources, (ii) inconsistencies in legal framework, (iii)

\footnotetext{
${ }^{52}$ According to the Ministry of Health (2016b) regulations, people who use drugs need to register at local clinics to participate in MMT. They must provide a confirmation letter, an introduction letter from local authorities, and personal identification (driving license or passport). They also need to fill in an application form. Later, all documents will be sent to the MMT clinic, local government and their families for consideration.
} 
social stigma and discrimination, (iv) political resistance, and (v) misleading perceptions of harm reduction.

\section{Insufficient Financial Resources}

External funding support has been crucial to the development of harm reduction in Vietnam (Heyman, 2013). Before 2013 it was estimated that almost all harm reduction funding came from international donors such as the World Bank, the United Kingdom's Department for International Development (DFID), Family Health International (FHI) or UNAIDS (Ahmed et al., 2014; Vuong, 2016). Yet since 2010, when Vietnam reached the level of a middle-income country, international organisations have begun to reduce funding (Morales, 2013). This deficit has undoubtedly increased resource challenges for the continuation of harm reduction interventions. For instance, in 2012 each injecting drug user was given around 180 needles and syringes; this dropped to 98 in 2013 (Vietnamese Government, 2014). Although the Vietnamese government has committed to increase funding, most has been spent on HIV/AIDS programmes, especially antiretroviral treatments (ART) rather than harm reduction per se (Ministry of Health, 2016b). This limited spend, alongside the gradual reductions from international donors, hampers the potential for harm reduction (Edington, 2016). This situation is not helped by the government's recent assertions that methadone is unaffordable and politically risky (Vuong et al., 2016).

\section{Inconsistencies in Legal Framework}

The discrepancy between the Drug Control Law and HIV Prevention Law is also one of the main stumbling blocks for an extension in harm reduction. According to the 2006 HIV Law, police forces should implement harm reduction. Following this 2006 development, the Vietnam National Assembly made amendments that allow police to be tolerant towards drug use, or to people carrying needles and syringes. Yet according to the 2006 Drug Control Law, police are required to fulfil tasks assigned by their leaders, meeting 'quotas' 53 on preventing and combating drug crimes, as well as sending people who use drugs to the rehabilitation

53 The internal police disciplinary procedures outline that each regional police force has to send a certain number of people who use drugs to treatment centres each year. This is a criteria to evaluate police performance (Ordinance on Commune Police, 2008: art 9.3). 
centres. Therefore, police officers try to send people who use drugs to compulsory treatment centres rather than to methadone treatment centres. Similarly, by virtue of the Administrative Violation Law 2012, ${ }^{54}$ police continue to round up people who use drugs for compulsory drug treatment centres (Windle, 2015). This means that even though illicit drug use has been decriminalised since 2009 , there has been no difference in the way that people who use drugs have been treated by police and local authorities (Vuong et al., 2012). These problematic tensions further confirm that Vietnam has not yet committed to harm reduction, and engages abstinence priorities. Such inconsistencies in law and policy also result in the lack of a multi-sectoral approach to harm reduction in Vietnam, as many agencies view harm reduction as the sole responsibility of $\mathrm{MOH}$ and international sectors (Ahmed et al., 2014).

\section{Social Stigma and Discrimination}

Many researchers like Edington (2016), Edington and Bayer (2013), and Windle (2015) argue that the high levels of stigma and discrimination within Vietnamese culture have served as a major hindrance for people who use drugs' access to harm reduction services. As noted earlier, Vietnamese people who use drugs are widely perceived as those who undermine moral traditions, have pathological failings, and are a threat to society. They are inevitably reluctant to engage in harm reduction because they fear the repercussions of disclosure, such as social blame or detention in an 06 centre (Edington \& Bayer, 2013). For example, if people who use drugs are methadone patients, they will be on the police list and will be at risk of punishment or incarceration (Tomori et al., 2014; Vuong et al., 2016). In fact, many local police make use of health service programmes as a mechanism to detect people who use drugs and then send them to the centres to meet quotas (Khuat et al., 2012).

As discussed previously, Vietnamese citizens often see the arrest or incarceration of people who use drugs as a positive sign for society (Reid \& Higgs, 2011). The stigmatisation also exists in treatment settings, as many officials are reluctant to work with people who use drugs (Kincaid \& Sullivan, 2010). This stigma makes people who use drugs feel despised and

\footnotetext{
54 The Administrative Violation Law 2012 categorises drug use as an administrative violation, for which people who use drugs face administrative detention for up to two years (MOLISA, 2015). This Law also restricts users from accessing needle exchange programmes and other harm-reduction interventions (Devaney et al., 2006; Hammett et al., 2007).
} 
rejected, which can lead to them becoming careless in protecting their own health and that of others, as well as preventing them from accessing harm reduction services again (USAID, 2011). Also, as noted above, if identified as a centre detainee, people who use drugs face challenges to socially re-integrate due to the high level of drug- and HIV-related stigma and discrimination in Vietnam (Human Rights Watch, 2011). As noted in Chapter Three, nonjudgemental attitudes are vital to implementing harm reduction, so if service providers still stigmatise and discriminate people who use drugs, authentic harm reduction is almost impossible. In general, the intense stigma and discrimination serve as major disincentives to access harm reduction services in Vietnam (Edington, 2016).

\section{Political Resistance}

Internationally, harm reduction is often conceived as a bridge connecting "human rights, public health, drug policy and HIV policies; yet this ideology has collided with local understandings of drug-related harm and harm reduction' (Edington, 2016, p. 8). In a country where drug use is regarded as both an individual failing and a major threat to the community, harm reduction is imagined as reducing harm to society as opposed to individuals (Edington, 2016). As argued earlier, the system of 06 centres remains one of the biggest barriers for harm reduction, but as Edington and Bayer (2013) and Vuong (2016) argue, many Vietnamese leaders have been unwilling to close the centres due to political interests over policy turf and government budget. If the 06 centres are scaled down, some key implementers (especially the 06 centre leaders, provincial politicians and bureaucrats) will lose their prioritised government budget (Vuong et al., 2017).

Another challenge is that adopting a discourse of individual human rights will not provide a stepping stone for harm reduction, as the wider community is always prioritised in Vietnam (Edington \& Bayer, 2013). Human rights issues are politically sensitive, as promoting problematic drug users' rights is generally opposed to the rights of the wider community and the government's purpose in controlling drug use problems. Yet it is essential to note that people who use drugs are part of the community, so considering that the rights of people who use drugs go against the rights of the community is contested. Further, the underestimation of people who use drugs' rights is not helped by common misperceptions that harm reduction philosophy promotes drug use. In general, these political factors consolidate the fact that 
although Vietnam has adopted the wording of harm reduction, it has not yet applied many of its important elements.

\section{Misleading Perceptions of Harm Reduction}

The different perceptions of 'harm' among international agencies, NGOs and Vietnamese authorities have also, as noted previously, led to different understandings and interpretations of the meaning of 'harm reduction'. For the international NGOs and donors who work in Vietnam, harm reduction refers to interventions that will reduce harms (primarily HIV) by reducing risky behaviours associated with drug use without forcing a reduction in drug consumption (Vuong et al., 2016). They have subsequently advocated and promoted needle exchange programmes and methadone treatment. At best, therefore, harm reduction is reregulated in the 2006 HIV law as a method to prevent the spread of HIV (Reid \& Higgs, 2011).

Meanwhile, Vietnamese leaders and policy-makers often perceive that reducing harm means ceasing drug use - they continue to see that incarceration, punishment, and re-education will reduce harm for society (Edington \& Bayer, 2013; Vuong et al., 2016). Officials see demand reduction and supply reduction as key methods to reduce harm, and they prioritise detoxification and compulsory drug rehabilitation centres to resist the social evils (Edington \& Bayer, 2013). Further, the media often reports that 06 centres reduce harms by helping people who use drugs to stay alive, have better health, and to reduce crime for society (The National Committee on AIDS Drugs and Prostitution Prevention and Control, 2011). Similarly, the prevalent police culture considers forced detoxification or detention as the only method to reduce the harms and threats that drug use and people who use drugs pose to society (Windle, 2016). Many police officers claim that providing needles and syringes to people who use drugs is 'showing the path to deer to run away' - in other words, that such provisions foster drug use (Khuat et al., 2012, p. 6). Further, police do not want to offer needles and syringes because they do not want to be misunderstood by the community who expect police to keep society 'clean', and free from drugs (Khuat et al., 2012). In reality, police usually use harm reduction programmes as mechanisms to control people who use drugs, and to meet quotas and the expectations of citizens (Khuat et al., 2012; Windle, 2015). In general, harm 
reduction is mistakenly understood by Vietnamese key professionals, and this takes time to change.

\section{Conclusion}

Vietnam's response to drug use has historically focused on deterrence through prioritising abstinence-based approaches, which include the suppression of drug supply and demand. In fact, Vietnam has successfully curbed opium poppy cultivation, but it has failed to control the use of illicit drugs. A wide range of costly supply and demand reduction measures have been implemented, but their effectiveness is limited and even counterproductive, resulting in discrimination and human rights violations.

Nonetheless, as a result of the HIV epidemic, harm reduction is now firmly rooted in Vietnam. It reflects a narrow public health perspective, with little attention being paid to the social, psychological and environmental disadvantages that hamper people who use drugs' community re-integration or their addiction treatment. Current repressive law enforcement, including the compulsory detention for people who use drugs, has combined with a range of barriers to authentic harm reduction strategies, including insufficient funding, social stigma and political factors. Where harm reduction is implemented, it is highly medicalised and designed to protect the wider community from HIV, rather than assisting people who use drugs (Edington, 2016; Edington \& Bayer, 2013).

Vietnam has committed to developing harm reduction, through a decrease in 06 centres and increase of harm reduction services; however, these shifts have not occurred. This raises the question of whether Vietnam really wants to implement harm reduction, or whether this stance is the result of international pressure. This thesis seeks to reflect upon these prevalent tensions between drug control (abstinence-based approaches) and harm reduction. In the following chapters, the study reviews how Vietnamese key stakeholders perceive drug use, drug addiction, and harm reduction strategies. It also examines how these perceptions affect the implementation of harm reduction in practice. In order to explore these perceptions, the research for this thesis has gained insight into how key professionals understand and respond to drug use and harm reduction. The next chapter reflects on the methodological approaches to the research and provides guidance on how this study was conducted. 


\section{Chapter Six: Methodology and Research Methods}

\section{Introduction}

The overall objective of this study is to examine how local and international stakeholders working in the 'drug field' perceive drug use, addiction and people who use drugs in Vietnam. Specific attention is also paid to how these professionals understand 'authentic' harm reduction, and how they apply its philosophy and principles in practice. The study is attentive to how stakeholders expose differences, conflicts, contradictions and commonalities in their perceptions of harm and its reduction. Importantly, it demonstrates the limitations of their perspectives about harm reduction and identifies the subsequent barriers that hamper harm reduction interventions. This ultimately paves the way for proposing practical solutions to improve the effectiveness of harm reduction strategies in Vietnam.

Critically exploring workers' perceptions is complex work, and this study, therefore, employed mixed qualitative and quantitative methods, specifically semi-structured interviews and a survey. Mixed methods were chosen because they allowed for an exploration of generic (quantitative) and more specific (qualitative) perspectives (Teddlie \& Tashakkori, 2010). On the one hand, by using quantitative methods, researchers can investigate how people generally think about drug use, people who use drugs and interventions. On the other hand, qualitative methods assist researchers to understand why some people agreed or disagreed with these viewpoints.

This chapter establishes the methodological approach for this study that engages both positivist and interpretivist approaches. It explains why the (positivist) survey and (interpretivist) interviews were chosen for this thesis, and illustrates how they were conducted in the fieldwork. The chapter also gives an overview of the research participants, and raises some of the personal and practical issues in doing research with 26 interviewees and 250 survey respondents, right across Vietnam. Further, it raises some of the ethical issues related to this study, including how privacy and confidentiality were assured in the completion of this thesis. Some practical issues related to accessing study participants are reflected in the chapter. The chapter also highlights the methods of analysis used to make sense of the collated data. Finally, it reveals my personal reflection of the PhD journey. 


\section{Methodology}

Comprehensively exploring and analysing Vietnamese professionals' perspectives on harm reduction in philosophy and practice is an original topic in Vietnam. It was, therefore, important to engage a flexible, mixed-methodological approach that could garner both generic and focused data. While the quantitative approach helped to identify general ideas and perceptions, the qualitative focus provided in-depth explanations for these perceptions. Nevertheless, as noted previously, the focus of this PhD thesis is to critically explore workers' understanding about drug use and their response to it, so qualitative approaches were prioritised.

Criminologists have long recognised the significance of combining qualitative and quantitative methods when they explore a topic (Chamberlain, 2013). The main reason for using mixed methods ${ }^{55}$ is that researchers can 'make use of the strengths of differing approaches' (Lanier \& Briggs, 2014, p. 189). For instance, as Wheeldon (2010) argues, the findings from quantitative research approaches can be used to strengthen those from qualitative ones, and vice versa. Mixed methods allow researchers to answer a broader range of research questions and to gain a deeper insight into social phenomena (Teddlie \& Tashakkori, 2010). Therefore, this integrated approach helped to critically explore how key stakeholders perceive harm reduction in Vietnam (Luong, 2017). For this fundamental reason, positivist and interpretivist approaches were chosen for this study.

In terms of methodology, there have been debates over whether or not positivist and interpretivist approaches can be combined together in one study (Giddings \& Grant, 2006). It is sometimes claimed that there is a confrontation between positivist and interpretivist

55 According to Wheeldon and Ahlberg (2012), the mixed methods approach involves the collection and analysis of both qualitative and quantitative data in a single study or in multiple phases of the research. Currently, there are six types of mixed methods, including: sequential explanatory design, sequential exploratory design, sequential transformative design, concurrent triangulation design, concurrent nested (embedded), and design concurrent transformative design (Creswell, 2007, 2012). The primary purpose of this study is to explore how people perceive drug use, drug users and drug policy, and so the concurrent triangulation design was chosen. That is, qualitative and quantitative data were collected concurrently in one phase. They were then analysed separately before being used to support particular arguments of the thesis. 
methodologies that makes them impossible to integrate into one single study (Della-Porta \& Keating, 2008; Maxwell \& Mittapalli, 2010). Yet as Giddings and Grant (2006) argue, depending on the research questions of a study, researchers can combine those approaches. The key research question of this $\mathrm{PhD}$ thesis relates to how key stakeholders perceive and respond to drug use in Vietnam in relation to harm reduction strategies, so using both positivist and interpretivist perspectives is necessary. This is because positivist approaches help to comprehend the general perceptions of study informants regarding drug use and corresponding responses in Vietnam, while interpretivist bases give insights into why they have such perceptions. It is important to note that all research approaches have their limitations, including mixed methods (Giddings \& Grant, 2006), that can be time-consuming and bring complexities in data analysis. Yet the benefits of mixed methods in gaining the general and specific perceptions of study participants ensured that it was the 'best fit' for this study.

Researchers who use a positivist philosophy argue that 'there is a reality that exists apart from our own perceptions of it', and the role of researchers is to have better understandings about this reality (Bachman \& Schutt, 2017, p. 11). In other words, 'there are factual realities that must be investigated through the rigorous process of scientific inquiry' (Gray, 2013, p. 21). Positivist approaches are also based on the assumption that, in social sciences, the researcher should separate their thinking and influences from the subjects they research (Della-Porta \& Keating, 2008). Maintaining an objective stance, the researcher should describe and analyse reality. Given my role as a police trainer in Vietnam, I usually followed the party-line (a drug law enforcement approach) regarding the response to drug use. Taking a positivist stance, I tried to be as objective as possible by strictly following the designed survey questions that were approved by the Victoria University Human Ethics Committee.

Nevertheless, a positivist approach has its own limitations, but this approach is still necessary for this study. One critique of positivism is that this approach lacks depth or it remains too general (Creswell, 2012). That is, it tends to use short questions and answers to gain general understanding, and this limits research participants in expressing their own perceptions or the reasons behind their answers. In addition, positivist approaches can be criticised for their limited validity. For instance, as Johnson (2014) argues, it is expected that some participants may choose random answers instead of providing authentic responses. This is likely the case 
if research participants are not really interested in the study, they are in a rush or they answer in terms of how they think their profession would demand it. Yet given the role of the positivist approach in this study (to only obtain general perceptions), these limitations can be overcome (study participants were also encouraged in this research to provide their detailed answers or further responses at the end of quantitative questions).

Positivists prefer quantitative methods such as social surveys or structured questionnaires because they have good reliability and representativeness (Bachman \& Schutt, 2017). This study surveyed 250 people, all of whom work for different agencies that respond to drug use problems in Vietnam, to understand their general perceptions of drug use and their corresponding responses to users. In this study, the majority of participants engaged in an ESurvey, while (for administrative ease) paper surveys were used for some police and MOLISA professionals. Relying on numerical data and statistics to comprehend the general ideas and perceptions of research participants (Lanier \& Briggs, 2014), quantitative approaches are particularly useful in the collection of data from large groups of people (Yeboah, 2008). In the study, several concepts and practices were measured and analysed by using quantitative methods (Lanier \& Briggs, 2014). For instance, a person's perception of harm reduction, the perceived effectiveness of a harm reduction programme or the most prominent principles of harm reduction strategies were surveyed (Appendix Three provides the survey questions). The survey helped, therefore, to explore participants' general thinking about drug use, people who use drugs, responses and harm reduction in Vietnam.

However, a quantitative approach cannot provide an in-depth explanation of why people think differently or the same (Choy, 2014). Therefore, an interpretivist approach was also used in this study. Interpretivists argue that 'reality is socially constructed and that the goal of researchers is to comprehend and explain what meaning people convey to this reality' (Bachman \& Schutt, 2017, p. 13). This methodological approach refutes the positivist philosophy, which deems that there is a concrete and objective reality that scientific methods can help to understand and explain (Lynch \& Bogen, 1997). Instead, interpretivists see that social reality is differently experienced and perceived; this requires different methods to evaluate individuals' actions and perceptions and to uncover how they interpret their surroundings. In other words, as Bachman and Schutt (2017) argue, researchers construct an image of reality based on their own preferences, prejudices and their interactions with others. 
Different people have different experiences and views about the same 'reality', and they understand and explain it from different angles (Brady \& Collier, 2010; Eliaeson, 2002). Thus, interpretivist qualitative methods, like the interviews used in this study, can tackle the questions that quantitative methods cannot encompass.

The advantage of qualitative methods is that they provide a depth of understanding of social phenomena, experiences and understandings in specific social, cultural and political settings (Bachman \& Schutt, 2012; Richard, 2013). For instance, by conducting interviews, I was more able to answer the study's research questions about how Vietnamese professionals perceive harm reduction or what the strengths and limitations of harm reduction strategies in Vietnam are, which led to a clearer understanding of how any limitations could affect harm reduction interventions in practice. Importantly, it should be noted that the purpose and objective of this research was to explore the perceptions, attitudes and understandings about drug use problems, so the study relied more heavily on these qualitative approaches.

\section{Research Methods}

\section{The Survey}

Surveys are some of the most common and widespread research tools in criminological research (Caulfield \& Hill, 2014; Yeboah, 2008). Basically, survey research involves the collection of information from a sample of individuals through their responses to predesigned standard questions (Yeboah, 2008). This research can be used to collect information concerning respondents' attributes, behaviours, attitudes and beliefs (Chamberlain, 2013) and, by doing surveys, general information can be obtained that illustrate commonalities of view on specific issues (Caulfield \& Hill, 2014). In addition, conducting a survey is costeffective because it measures opinions from a broad spectrum of individuals without substantially increasing research time or costs (Schutt, 2012). Thus, in order to explore the differences and commonalities in the perceptions of key stakeholders on drugs, drug dependence, treatment, and harm reduction approaches, a survey method was chosen.

The survey questions, which were directed at the research questions and objectives of the study, aimed to explore how professionals perceive drug use, drug users, responses to drug use, and harm reduction. The survey questions were mainly obtained from the extant literature by developing questions from the ideas presented in literature. During the literature 
review, special attention was paid to how other researchers formulated the main principles of harm reduction and abstinence in relation to drug use, users, treatment or drug policy. Designing and developing survey questions was a selective and ongoing process to make sure they were linked with and helped to answer research questions. That is, the survey questions were selected from the key principles and differences between abstinence and harm reduction in terms of conceptualising drugs, drug use, viewing those take drugs, and the responses to drug use and users. All survey questions were then further developed from intensive discussions with my supervisors, and later, they were finalised by the researcher (formatting and translating into Vietnamese). To ensure the reliability and validity of the survey, piloting was conducted with my police friends $(n=2)$ to check whether or not the survey was easy to do, and if the translated questions were clear and understandable. In general, there were no significant changes in the survey questions after the piloting.

Later, the survey was conducted as follows. Closed questions (in English for international survey participants and in Vietnamese for locals) were used with a final open-ended question to allow participants to add any additional comments they wished (see Appendix Three). I also asked survey respondents whether they would be interested in being interviewed (for recruitment purposes). Across most questions, a Likert scale was developed that included five levels of agreement: strongly agree, agree, 'undecided's6, disagree, or strongly disagree. Respondents chose their 'agreement' in response to the pre-defined set of questions, such as "how far do you agree/disagree with the following statement: Most people who take drugs do so recreationally and rationally".

The survey enabled the statistical comparison of the answers from a large number of respondents. I could see whether participants had a particular view of harm reduction, and, if so, how (if at all) this view might be in conflict with the philosophy and principles of authentic harm reduction. In order to review the survey, and to minimise mistakes or inappropriate questions, a pilot survey was conducted in advance at the Faculty of Drug Law Enforcement, the Police College, Ho Chi Minh City. This pilot provided information about how easy and motivating the survey was to complete, as well as how long it took (Costley, Elliott,

${ }^{56}$ The phrase undecided in the survey referred to 'Neither agree nor disagree'. 
\& Gibbs, 2010). After piloting, the survey was conducted in 25 organisations and agencies (Appendix Four lists the surveyed groups).

Research participants were emailed the survey or provided with a web link to 'vuw.qualtrics.com'. ${ }^{57}$ Due to the 'confidential rule's8 in Vietnamese police forces, police respondents completed a paper copy of the survey on their own, either in their office or at home. These surveys were collected in person. Professionals working in other agencies mainly did an e-survey from the 'vuw.qualtrics.com' link that was sent via email. The table below shows the distribution of the 250 survey participants in terms of the work setting, gender and work location (see Table 6.1), (fully reported in Appendix Nine).

57 This site was provided by Victoria University of Wellington (VUW) to support VUW's students in conducting research surveys.

58 The confidential rule is a compulsory regulation in the Vietnamese police force. It establishes that police officers cannot send emails or engage in interviews with those who are not working for MPS. Therefore, I had to conduct the survey in person and to collect them from police offices. 
Table 6.1: The work setting, gender and work location of survey respondents $(n=250)$

\begin{tabular}{|c|c|c|c|c|c|}
\hline \multicolumn{3}{|l|}{ Work setting } & \multicolumn{2}{|l|}{ Gender } & \multirow{3}{*}{$\begin{array}{l}\text { Total } \\
81 \\
\end{array}$} \\
\hline & & & \multirow{2}{*}{$\begin{array}{l}\text { Male } \\
75\end{array}$} & \multirow{2}{*}{$\begin{array}{l}\text { Female } \\
6\end{array}$} & \\
\hline Law enforcement sectors & Work location & Ho Chi Minh City & & & \\
\hline & & Hanoi City & 21 & 7 & 28 \\
\hline & Total & & 96 & 13 & $\begin{array}{l}109 \\
(43.6 \%)\end{array}$ \\
\hline \multirow[t]{3}{*}{ Public health sectors } & \multirow[t]{2}{*}{ Work location } & Ho Chi Minh City & 7 & 21 & 28 \\
\hline & & Hanoi City & 2 & 7 & 9 \\
\hline & \multicolumn{2}{|l|}{ Total } & 9 & 28 & $\begin{array}{l}37 \\
(14.8 \%)\end{array}$ \\
\hline \multirow[t]{3}{*}{ Social work sectors } & \multirow[t]{2}{*}{ Work location } & Ho Chi Minh City & 13 & 9 & 22 \\
\hline & & Hanoi City & 7 & 7 & 14 \\
\hline & \multicolumn{2}{|l|}{ Total } & 20 & 16 & $\begin{array}{l}36 \\
(14.4 \%)\end{array}$ \\
\hline \multirow[t]{2}{*}{ Local authorities } & Work location & Ho Chi Minh City & 23 & 6 & 29 \\
\hline & \multicolumn{2}{|l|}{ Total } & 23 & 6 & $\begin{array}{l}29 \\
(11.6 \%)\end{array}$ \\
\hline \multirow[t]{3}{*}{ International sectors } & \multirow[t]{2}{*}{ Work location } & Ho Chi Minh City & 5 & 5 & 10 \\
\hline & & Hanoi City & 7 & 7 & 14 \\
\hline & \multicolumn{2}{|l|}{ Total } & 12 & 12 & $\begin{array}{l}24 \\
(9.6 \%) \\
\end{array}$ \\
\hline \multirow[t]{3}{*}{ Others } & \multirow[t]{2}{*}{ Work location } & Ho Chi Minh City & 2 & 10 & 12 \\
\hline & & Hanoi City & 1 & 2 & 3 \\
\hline & \multicolumn{2}{|l|}{ Total } & 3 & 12 & $\begin{array}{l}15 \\
(6 \%) \\
\end{array}$ \\
\hline \multirow[t]{3}{*}{ Total } & \multirow[t]{2}{*}{ Work location } & Ho Chi Minh City & 125 & 57 & $\begin{array}{l}\frac{182}{(72.8 \%)} \\
\end{array}$ \\
\hline & & Hanoi City & 38 & 30 & $\begin{array}{l}\frac{68}{(27.2 \%)} \\
\end{array}$ \\
\hline & \multicolumn{2}{|l|}{ Total } & $\begin{array}{l}163 \\
(65.2 \%)\end{array}$ & $\begin{array}{l}87 \\
(34.8 \%)\end{array}$ & 250 \\
\hline
\end{tabular}

There are some noticeable points about the survey participants. In terms of location, 182/250 survey participants were in Ho Chi Minh City, while the remainder $(n=68)$ work in Hanoi City. The survey was conducted in Hanoi City to allow for greater input from those working at Ministerial and international levels, so the number of participants was smaller than Ho Chi Minh City (local level). Regarding gender (highlighted in red), the number of male participants 
(163) was higher than that of female participants (87). This is because male staff generally outnumber their female counterparts in most work sectors ${ }^{59}$ within Vietnam, but especially in law enforcement and social work sectors.

With respect to work setting (highlighted in blue), there was a bias as 109/250 respondents work for law enforcement sectors compared with others working in different sectors. This over-representation relates to how law enforcement sectors play an essential role in addressing drug use, controlling people who use drugs and other drug use related interventions within Vietnam. Another reason would be that, as a police officer, I am able to receive higher commitment from the police hierarchy to support the work if I conduct surveys with the police. Yet whatever the reason, this study is inevitably distorted along the lines of work-based setting. Regarding other sectors, the numbers of participants working for public health sectors, local authorities, social work and international sectors were fairly equal.

\section{Interviews}

In-depth interviews are an appropriate data collection method for the purpose of finding out people's experiences, thoughts and feelings (Bachman \& Schutt, 2012; Boyce \& Neale, 2006). In this study, interviews were chosen for several reasons. Firstly, this method helped to gain deeper insights into people's perceptions of drug use, people who use drugs, and on how they respond to drug use as well as harm reduction strategies. As a result, a more comprehensive picture of Vietnam's drug policy in conjunction with harm reduction strategies was explored. For instance, some issues related to the limitations, conflicts or barriers of current laws, policies and practices in implementing harm reduction strategies could not be explained in a survey but, through interviews, respondents articulated their thoughts on these issues. Secondly, the interviews enabled new insights into the topic when respondents had more time to express their ideas. Finally, given that one objective of the research was to propose recommendations to improve harm reduction strategies in Vietnam, interviews also allowed respondents with a wealth of practical experience and knowledge to raise suggestions.

Similar to the process of developing the survey questions, the interview questions derived from the wider literature related to harm reduction and abstinence philosophies. The

${ }^{59}$ Given Vietnamese culture and society, women are mainly tasked with household work, so the proportion of Vietnamese women who work for government agencies is low. 
questions were firmly associated with the research questions and objectives of the study. All interview questions were then carefully reviewed by my supervisors before formatting and translating into Vietnamese. The translation process (from English into Vietnamese) was conducted by the researcher. The meanings of the interview questions remained consistent after translation. In order to make sure the questions were easy to understand after translation, and to gauge whether or not they were politically sensitive, all interview questions were tested with my Vietnamese police colleagues $(n=2)$ (interviewing them in Vietnamese). All reported that the interview questions were acceptable and not too sensitive or problematic in any way. After the trial, no significant changes were made in terms of the content of the questions.

To mitigate the work setting bias among survey participants, interviews were conducted with 26 participants (9 females and 17 males), from a wide range of roles and work settings, from the city, ministerial and international levels (See Table 6.2 below). 
Table 6.2: The interviewees

\begin{tabular}{|c|c|c|c|}
\hline No. & The work setting & Role & Work Location \\
\hline 1 & Local Drug Law Enforcement Unit & Police & Ho Chi Minh City \\
\hline 2 & Police College & Police Trainer & Ho Chi Minh City \\
\hline 3 & Department of Drug Crime Prevention - MPS & Senior investigator & Ho Chi Minh City \\
\hline 4 & Methadone Clinic & Doctor & Ho Chi Minh City \\
\hline 5 & Methadone Clinic & Consultant & Ho Chi Minh City \\
\hline 6 & $\begin{array}{l}\text { Local Social Vices Prevention and Combat } \\
\text { Unit - MOLISA }\end{array}$ & Social worker & Ho Chi Minh City \\
\hline 7 & $\begin{array}{l}\text { Local Social Vices Prevention and Combat } \\
\text { Unit - MOLISA }\end{array}$ & Director & Ho Chi Minh City \\
\hline 8 & Local Police & Police officer & Ho Chi Minh City \\
\hline 9 & Local Authority & Local leader & Ho Chi Minh City \\
\hline 10 & Harm Reduction Office & Specialist & Ho Chi Minh City \\
\hline 11 & Harm Reduction Office & Specialist & Ho Chi Minh City \\
\hline 12 & $\begin{array}{l}\text { Department of Social Vices Prevention and } \\
\text { Combat - MOLISA }\end{array}$ & Social Worker & Ho Chi Minh City \\
\hline 13 & Drug Treatment Centre & Doctor & Ho Chi Minh City \\
\hline 14 & Expertise France $^{60}$ (in Vietnam) & Social worker & Ho Chi Minh City \\
\hline 15 & Expertise France (in Vietnam) & Social worker & Ho Chi Minh City \\
\hline 16 & $\begin{array}{l}\text { The National Committee for AIDS, Drugs, and } \\
\text { Prostitution Prevention }\end{array}$ & Government official & Hanoi City \\
\hline 17 & The Judicial Committee of Vietnam Congress & Government official & Hanoi City \\
\hline 18 & $\begin{array}{l}\text { The Department of Social Vices Prevention } \\
\text { and Combat - MOLISA }\end{array}$ & Social worker & Hanoi City \\
\hline 19 & $\begin{array}{l}\text { The Department of Social Vices Prevention } \\
\text { and Combat - MOLISA }\end{array}$ & Social worker & Hanoi City \\
\hline 20 & The Department of Harm Reduction $-\mathrm{MOH}$ & Official & Hanoi City \\
\hline 21 & The Drug Law Enforcement Office - MPS & Police & Hanoi City \\
\hline 22 & $\begin{array}{l}\text { Institute of Labour and Social Affair Training } \\
\text { - MOLISA }\end{array}$ & Social worker & Hanoi City \\
\hline 23 & $\begin{array}{l}\text { The Joint United Nations Programme on } \\
\text { HIV/AIDS (UNAIDS), Vietnam }\end{array}$ & International worker & Hanoi City \\
\hline 24 & Family Health International (FHI), Vietnam & International worker & Hanoi City \\
\hline 25 & $\begin{array}{l}\text { United Nations Office on Drugs and Crime } \\
\text { (UNODC), Vietnam }\end{array}$ & Specialist & Hanoi City \\
\hline 26 & $\begin{array}{l}\text { Substance Abuse and Mental Health Services } \\
\text { Administration (SHAMSHA), Vietnam }\end{array}$ & International worker & Hanoi City \\
\hline
\end{tabular}

Regarding interviewees' recruitment, there were three main methods. The first, as noted earlier, was from the survey. At the end of the survey, respondents were asked whether they

${ }^{60}$ The agency promotes French public expertise with a view to building public policies meeting the institutional, economic, demographic, social and environmental challenges of partner countries. It also supports Vietnam to implement some harm-reduction interventions. 
were interested in further participating in an interview. Those who agreed were interviewed $(n=5)$. The second method was purposive sampling. Purposive sampling was used to ensure representation across the three local, ministerial and international groups, and the participants who had a range of experience in drug policy, drug law enforcement and working with people who use drugs $(n=10)$. 'Snowball sampling' ${ }^{61}$ was the third strategy. This approach was used relatively effectively and yielded eleven research participants. One example of successful snowballing was that an interviewed doctor introduced me to a consultant who had worked with people who use drugs for over ten years.

Semi-structured questions (in English for international professionals and in Vietnamese for locals) were designed for the interview. These questions asked participants about their perception of drug use, people who use drugs, drug use responses, and harm reduction strategies (see Appendix Five for the interview questions). Some did not answer questions that were about politically sensitive topics or those questions that were not considered relevant to their position. For instance, some participants from MOLISA refused to answer questions on compulsory treatment centres. In addition, after some participants deemed that some questions needed to be clarified, changes were made to the structure or content of the questions.

Each participant was contacted by email and/or telephone and they received a formal invitation letter with the main contents of the interviews in advance (alongside the explanation about the participant information sheet and informed consent). The interviews were carried out in a private room or a negotiated place, to avoid intrusion and interference from other people. Each participant was explained about the information sheet and informed consent. Most interviews were conducted in about 60 minutes. All participants were reminded that they could leave the interview at any time without needing to give a reason. After the interview, each participant was given a gift to thank them for their participation.

61 Participating respondents were asked which subsequent respondents should be involved in the research (Gray, 2009). 


\section{Ethics}

Ethics approval was received from the Victoria University of Wellington Human Ethics Committee for this research (Ethics Approval No: 22558, issued 18 December 2015) (see Appendix Six). There were three main ethical issues in this study. The first was how to minimise harm to participants. In order to mitigate potential harm for participants' professional reputation, I endeavoured to reassure them about privacy and confidentiality. The information gained from surveys and interviews was kept confidential and was only accessible to myself and my supervisors. After completing the thesis, these interviews and surveys will be destroyed.

The second issue was related to 'informed consent' (Appendix Seven). To conduct the interviews and survey, I obtained written permission from sector leaders where I planned to do my research. All participants received an 'information sheet' form (Appendix Eight) and consent in advance. The nature and purposes of the research were explained in detail before commencing interviews and surveys.

The final issue was privacy. All participants were informed about their rights, they were reminded that their participation was voluntary, and told that no names would be documented in the study. Only their general job role was noted. In order to allow participants to speak freely and be honest (given the level of controls over information distribution in some Vietnamese organisations - particularly the police) the interview locations were negotiated. Therefore, every effort has been made to protect the confidentiality of the participants in this study.

\section{Practical Issues}

A primary issue was that of commuting to locations for the surveys and interviews. I returned to Vietnam from New Zealand, a day's flight away, for the field trip. At first, the research survey and interviews were carried out in Ho Chi Minh City, where I previously lived for 13 years. ${ }^{62}$ Despite knowing the city, it is always challenging to travel around - motorbikes are widespread and traffic congestion is severe. Surveys and interviews were conducted in District 6 and District 8 where there are the highest number of registered people who use

${ }^{62}$ Ho Chi Minh City is the biggest city in Vietnam with a population of around 10 million. 
drugs in the city. It took more than one hour to travel to reach police stations in these districts. Another difficulty was that even though appointments were booked in advance, police leaders were not always available because of many unexpected duties. I travelled three times to the two police stations in Districts 6 and 8 before the surveys and the interviews could be completed.

Conducting surveys and interviews in Hanoi City, a city with over 8 million people, was also a challenging experience because of traffic. It would have been unaffordable, and timeconsuming if the commute had been done via taxi. The traffic was chaotic, so borrowing a motorbike from a friend meant travelling across the city was more easily accomplished. Travelling around $100 \mathrm{~km}$ per day in Hanoi by motorbike in the hustle and bustle of the city was not easy work, demonstrating one of the practical issues that affected the collection of fieldwork.

Additionally, for some interview participants, appointments could not be made in advance because they were introduced by other participants. For example, to interview two essential participants who worked for the Judicial Committee of Vietnam Congress and the National Committee for AIDS, Drugs, and Prostitution Prevention, my field trip had to be extended by thirteen days. As interviews with all other participants had already been completed, this wait was inconvenient and frustrating. Eventually, after interviewing both participants, I felt really fulfilled as they gave a deeper understanding into why Vietnam has a punitive-based approach towards drug use and people who use drugs and how harm reduction is undermined in Vietnam as a result.

\section{Access}

The survey was fairly straightforward to conduct, except for access to two agencies belonging to MOLISA (described below). Most agencies were happy to participate in the survey. The majority of participants completed the survey online, so the results were received straight after they had been completed. Some agencies, mainly from MOLISA and Police sectors, received the survey papers and then allocated surveys to their officials, which were collected after one or two days (as detailed above). The biggest challenge was from the Department of Social Vices Prevention and Combat in Hanoi City, and a Social Vices Prevention and Combat 
Unit in Ho Chi Minh City. They took a long time to consider and complete the survey. Although there were some challenges, enough quantitative information was collected for data analysis. In term of interviews, accessing those who work for police forces, public health sectors and international sectors was relatively straightforward (maybe because I am a police officer). The main challenge, here, was that they were extremely busy with their work, so there was a need for flexibility and patience. Having worked in the police force for 13 years, it was not too challenging to obtain consent for the interviews in police units, such as district police stations, the Department of Drug Law Enforcement, or police schools. Also, there were no significant challenges when trying to contact participants who worked for the public health sectors. For instance, immediate consent was forthcoming from doctors and professionals, who worked in harm reduction clinics in Ho Chi Minh City, after the research was explained to them.

Alongside these positive interactions, I encountered many access challenges. The first occurred when interviewing the very first participant, who is the leader of a local drug law enforcement unit. The first impression was that he looked formidable. After introducing myself and giving him the ethics form and relevant information, it became clear that he knew my workplace, and he even worked with some of my police friends. After that, the conversation progressed smoothly, until I mentioned that the interview would be recorded. At this point, his face darkened with clear worries. He said I could ask anything and he would speak slowly so that his words could be written down, but the recording should not be conducted. At the time, I did not know how to deal with this dilemma, and was worried, especially as he was my first interviewee. I again firmly insisted that his information and the interview would be kept confidential and no one would have access, except my supervisors and me. However, he was still hesitant and I said to him, 'alright, if you are not ready, we can stop our conversation and we can talk about other topics. I cannot write down when I am interviewing people, so I think I am going to find another option'. After a few seconds of hesitation, he said 'ok, I will take a risk with you, but the information provided will align and be quite consistent with Vietnamese drug policy so far'. The interview took more than one hour and his information was valuable, generally reflecting on how ordinary people and the police think about the issues of drug use and control.

The main challenge, however, came from those working for MOLISA both at local and ministerial levels because they are responsible for maintaining the 06 system, which would 
be asked in the interview sections. I made an appointment with a MOLISA official to interview. I visited his office a few times before we could arrange an appointment. As his boss was busy, he was allocated to talk to me. My feeling was that he was generally worried and scared of disclosing information relating to 06 centres and how the Unit should respond to people who use drugs. His answers were short, simple and seemed to follow the 'party line' - which is based on punitive approaches. When he was asked about the weaknesses of 06 centres he politely declined to answer, and just recommended that I 'pay a visit to the centres' 63 in order to receive correct answers. Very little information was gathered because he was so cautious. Also, it was very difficult to make contact with people who were working in the Department of Social Vices Prevention and Combat in Ho Chi Minh. This was probably because they were wary of revealing something related to how they address drug use problems and treat people who use drugs. In Ho Chi Minh, this is so sensitive that not many people in this department are ready to talk and share their thoughts. Despite my attempts to clearly explain the study, and to reiterate my commitment to confidentiality, a cautious approach was widespread in this department. Understandably, the interviewees refused to answer many questions, especially when it came to 'the existence of 06 system'. A leader urged me that 'some western countries have tried to force our country to change drug policy, so you need to be very cautious'. This makes sense when they need to follow 'the party line', an important rule, given their roles working as government officials.

For those who worked for the Department of Social Vices Prevention and Combat and MOLISA, the structure and some minor content of questions were rearranged to encourage their participation (See Table 6.3). I asked the questions in a softer way, hoping the participants may give their answers on the 06 system. Although these changes were made, unfortunately, some still refused to answer questions about compulsory treatment centres. This demonstrates what a sensitive issue these centres are, and how this affects progress towards authentic harm reduction in a Vietnam context.

63 Unfortunately, permission could not be obtained from the Department of Social Vices Prevention and Combat, Ho Chi Minh City, to visit, conduct surveys and interview in compulsory treatment centres. Some key reasons are explained in the following paragraph. 
Table 6.3: The initial and changed questions on compulsory treatment centres

\begin{tabular}{|l|l|}
\hline Initial questions & Amended and revised questions \\
\hline - What are your thoughts on the effectiveness of & - We all see that addiction should be a concern \\
coercive treatment? & $\begin{array}{l}\text { and that we have some types of treatment, so } \\
\text { do you think that sending addicts to compulsory }\end{array}$ \\
- In terms of approach, how would you describe & $\begin{array}{l}\text { treatment centres is an option? And why? } \\
\text { the approach of } 06 \text { centres? }\end{array}$ \\
- To what extent are 06 centres effective? & $\begin{array}{l}\text { - Why should we keep compulsory treatment } \\
\text { centres? }\end{array}$ \\
- What do you think are the strengths and & $\begin{array}{l}\text { - Can you think of any improvements that should } \\
\text { weaknesses of } 06 \text { centres? }\end{array}$ \\
& $\begin{array}{l}\text { be made to enhance the effectiveness of } \\
\text { compulsory treatment centres? }\end{array}$ \\
\hline
\end{tabular}

In general, although there were many challenges in contacting and interviewing study participants, 26 interviews were conducted, and they provided rich qualitative data for analysis.

\section{Data Analysis}

SPSS software was used to analyse the quantitative data. Question responses were analysed from two main perspectives. The study was to look at each question individually by utilising the 'frequencies' function of 'descriptive statistics' in SPSS. Some figures/diagrams (using quantitative data) were used to illustrate how the participants agreed/disagreed or were undecided in response to certain survey questions. These figures were chosen to back up study arguments and qualitative data. For instance, the majority of interviewees favoured methadone programmes, and this fact was supported by quantitative data in which over $80 \%$ of survey respondents agreed or strongly agreed with these programmes.

The qualitative data emphasised in this study has been analysed systematically to generate themes and findings. I have drawn upon grounded theory (GT), particularly constructivist grounded theory, as the foundation for the data analysis. Grounded theory, developed by Glaser and Strauss in 1967, is an inductive approach which aims to develop new theories from data collection and analysis (Costley et al., 2010). Generally, there are four main versions of 
grounded theory, including classic grounded theory ${ }^{64}$, constructivist grounded theory, feminist grounded theory ${ }^{65}$, and Straussian Grounded Theory ${ }^{66}$. This study was informed by $\backslash$ constructivist grounded theory. Constructivist grounded theory was developed by Charmaz $(1996,2006,2014)$. Its key component is that concepts are constructed (Alexandrescu, 2014). That is, the process of constructing themes begins with specific questions on a particular substantive area, and then a review of the literature is conducted to determine what has been done before the research (Alexandrescu, 2014 ). Next, new themes emerging from data are compared with the literature to construct new theories. As Charmaz (2014) explains, constructivist grounded theory is unique in its ability to create concepts by coding and comparing data constantly with previous literature. As a result, the researcher can gain deeper and richer insights or can make sense of the data in order to generate new themes ${ }^{67}$ or theories. Smith (2015), Charmaz (2014), and Timmermans and Tavory (2012) have all highlighted that constructivist grounded theory is among the most useful form of analysis for social and qualitative data.

Specifically, three key tenets of a constructivist grounded theory approach have influenced how qualitative data have been analysed in this study. First, key themes emerged from the data, rather than being derived from a pre-established hypothesis. The qualitative interview data were analysed to identify the categories or themes that helped to answer the research

\footnotetext{
${ }^{64}$ Classic grounded theory is an inductive approach which aims to find out new theories from data collection and analysis, not to test existing hypotheses or theories about a situation (Costley, Elliott, \& Gibbs, 2010). This means that a theory emerges from the data, not from the literature. Meanwhile, constructivist grounded theory is based on reviewing literature first and then identifying and comparing with themes emerging from data in order to construct new theories.

${ }^{65}$ Feminist grounded theory was developed in recognition of the androcentric bias in most research, and to ensure that women's voices were heard (Wuest 1995).

66 Strauss and Corbin (1990) developed more detailed and advanced grounded theory procedures and techniques. They incorporated the coding structure with more advanced procedures on how to code and structure the data. Their methods involve both deductive and inductive processes.

67 Although there were some small studies related to drug policy in Vietnam, and they provided insight into some of the problems related to Vietnamese drug policy, most themes emerging from this study were new in comparison, and constructed throughout the data analysis process. For instance, the interview and survey data about social and cultural factors, barriers to harm reduction, or how to implement harm reduction were new themes in this research, and they were only established after the data were analysed.
} 
questions (Charmaz, 2006, 2008, 2011). For instance, through coding processes, some key themes (such as that: drug use inevitably leads to addiction; drug use causes crime; or, 06 centres are a form of Vietnamese harm reduction) contributed to a conclusion that Vietnamese people propel strong myths about drugs and drug policy. Second, data were constantly compared with emerging categories (Hallberg, 2006). Thus, emerging categories/themes were identified and named, and then compared with other themes to find thematic connections (Brink et al., 2006; Engward, 2013). By doing so, conceptual connections and theoretical frameworks were grounded in the data (Hallberg, 2006). For example, making comparisons between how Vietnamese professionals perceive drug use allowed this study to conclude that drug use is widely seen a social evil rather than a public health issue, which explains why abstinence-based mind-sets remain widespread in Vietnam. The third tenet is the selection of 'slices data'. This phrase was coined by Glaser and Strauss (1967) to show the fact that different data provides researchers with different meanings (Urquhart, 2012). This means that some slices of data can generate a theory or theme while others provide another. For instance, some data related to how study participants perceived addiction contribute to identifying the simultaneous existence of moral and brain disease models of addiction in Vietnam, while the data about official responses to drug use helps to reaffirm punitive drug policies. In general, the data analysis process was influenced by a constructivist grounded theory approach, ensuring that I sought to analyse qualitative data in a systematic way.

It is important to note that this study has not developed from a grounded theory approach, as this would require a more limited researcher input into the questions/ideas that have guided the research. However, constructivist grounded theory principles in the analysis stage have been an important and suitable reminder of the need to be attentive to the new ideas, concepts and perspectives that emerge from the data analysis. That is, some principles have been useful for this study, because they made me mindful of coding data, developing, checking, and integrating theoretical categories, and writing analytic narratives throughout interviews (Charmaz, 2014).

The philosophical insights of grounded theory influenced my data analysis but, on a practical level, 'thematic data analysis'68 helped me to gain insights into interviewees' thoughts or

68 Thematic analysis is a popular method for qualitative social sciences (Boyatzis, 1998), and it is used to identify, analyse and report patterns (themes) within data (Braun \& Clarke, 2006). 
perceptions. When practically analysing qualitative data, I based it on the six main steps of thematic analysis proposed by Braun and Clarke (2006), including: familiarising yourself with your data; generating initial codes; searching for themes; reviewing themes; defining and naming themes; and finally producing the report. Due to a high amount of information collected from 26 interviewees, this thematic analysis method was used with the aid of Nvivo software (still going through six above steps). Nvivo allows the researcher to usefully organise, analyse and identify themes from quantitative data quicker, but still in an organised way. Also, since qualitative data is text-based, the cornerstone of analysing the data is the coding process, for which NVivo is really helpful (Hilal \& Alabri, 2013).

The first step in qualitative analysis for this study was to transcribe the 26 interviews into document form, both in English (for international professionals) and Vietnamese (for local professionals). Next, these documents were imported into Nvivo software for coding. Before coding, each interview transcript ${ }^{69}$ was read thoroughly, so I could understand the general ideas and concepts and familiarise myself with the data. During the data analysis process, interviews were then organised categorically and chronologically for coding purposes (Hill, 2005). The 'coding'70 of each interview transcript followed. Codes often included phrases, sentences or the entire paragraph associated with research objectives (e.g. exploring key stakeholders' perceptions regarding drugs, drug use, users, addiction, responses, or harm reduction). For example, regarding how interviewees perceived people who use drugs, some important phrases were coded such as 'diseased people', 'violating social conventions and morality', or 'a weak-willed individual'.

These phrases, sentences and paragraphs were then combined together in order to identify key themes (e.g. perceptions of people who use drugs) (Bound, 2011). Another example is

Thematic analysis method is a 'rigorous thematic approach can produce an insightful analysis that answers particular research questions' (Braun \& Clarke, 2006, p. 97). Thematic analysis allows researchers to complement the research question by facilitating an investigation of the interview data (Judger, 2016). 'Thematic analysis' was used with the support of Nvivo, which is much faster and more systematic. In the findings chapters, the key themes are highlighted in the introduction section.

${ }^{69}$ For Vietnamese transcripts, the English translations were undertaken by me, and I only translated what people said into English. The key meanings of the codes in Vietnamese and English are the same. There was some help from my supervisors in terms of correcting English, but the contents remain unchanged.

${ }^{70}$ The coding process was conducted by the researcher with the aid of NVivo software. 
that some sentences aligned with 'key principles of harm reduction' (e.g. the majority of people use drugs, and users did not cause criminal behaviour) were grouped together, while others related to 'myths' (e.g. people who use drugs are an underlying cause of crimes) were also linked together. Finally, important quotes were selected to exemplify key themes in the findings. In general, three key themes emerged. These reflected (i) study participants' perceptions of drug use, (ii) responding to drug use, and (iii) harm reduction. Many subthemes were also identified to support these key themes, such as myths about drug use, addiction, people who use drugs, harms, demand and supply reduction approaches, understanding harm reduction, and attitudes towards harm reduction practices. These key themes and sub-themes are further illustrated across the following chapters.

\section{Conclusion}

This chapter has provided an overview of the methodological approach and research methods used to carry out this PhD study. With a mix of qualitative and quantitative methods, the following chapters reflect on the findings from the survey with 250 respondents and 26 semistructured interviews in two main cities, including Ho Chi Minh City and Hanoi City. Given the mixed approaches, the findings demonstrate the general perceptions of research participants about drug use, responses to drug use and harm reduction, while also explaining why participants had particular perceptions.

In describing the personal and practical issues during the field trip and the challenges to access data, the research process has reinforced the fact that Police and MOLISA officials are generally inaccessible, especially when it comes to discussing compulsory treatment centres. They are less likely to openly share their thoughts than other workers from health or international sectors. The research process gave the first indications of some of the differences between different professionals (for example, in relation to their knowledge, agendas, positions on drug use, users or harm reduction), which are further presented in the following chapters. 


\section{Chapter Seven: Findings on Perceptions of Drug Use}

\section{Introduction}

This chapter presents the findings from 26 interviews and the survey with 250 professionals who work around the issue of drug use in Vietnam. Specifically, it shows how these professionals perceive drugs, drug use, addiction, people who use drugs, and the harm caused by drug use. The findings of quantitative data (fully reported in Appendix Ten) and qualitative ones indicate that numerous myths exist about all these issues. Most study participants believed illicit drugs are the most harmful, and some even thought that cannabis causes death. Further, only a few survey respondents and interviewees thought that illicit drugs can be used recreationally. Most perceived that using these drugs inevitably results in addiction as well as crime. All these myths demonstrate that abstinence-based mind-sets and judgemental attitudes remain popular among Vietnamese key stakeholders.

The majority of interviewees and survey respondents regarded drug use as a public health problem, and addiction as a brain disease associated with genetic factors. A few interviewees indicated the importance of social and psychological elements in shaping addictive behaviours. These ideas generally reflected an abstinence-based perspective. However, many research participants continued to view drug use as a social evil that must be eliminated. They often saw that those taking drugs are weak and have psychological problems. As a result, the majority of interviewees thought that the harms of drug use are caused by drugs and those who take drugs, even though some interviewees had wider perspectives of harm, which are closer to an authentic harm reduction philosophy. These findings are important in understanding: why people who use drugs have been judged due to their drug use behaviour; why they are subjected to punitive measures in Vietnam; and why harm reduction is often equated to reducing drug use and controlling people who use drugs within Vietnamese drug policy.

Overall, the findings demonstrate that many study participants had misunderstandings about drugs, drug use, people who use drugs and harm, and these misunderstandings are influenced by political and social factors rather than evidence. A common perception is that the harms of drug use are solely caused by people who use drugs. This misperception reaffirms 
judgemental attitudes towards people who use drugs. Also, the misunderstanding ignores the complexity of drugs, or the role of social, environmental and legal factors in creating harms. Such perceptions reflect an abstinence-based stance and explain why Vietnam has implemented pseudo harm reduction.

\section{Myths}

The general perceptions among interview participants about drug use, addiction and people who use drugs were similar to the global understandings presented in Chapter Two. Interviewees and survey respondents $(n=250)$ tended to see drug use as a public health matter and addiction as a brain disease, but the way they perceived people who use drugs was not aligned with a brain disease model of addiction. In fact, the attitude towards people who use drugs generally reflected a moral model of addiction in which people who use drugs are treated as a specific group that needs to be controlled. The findings of this study were similar to the previously discussed myths that have underpinned abstinence-based approaches. Both the quantitative and qualitative results demonstrate numerous myths and flawed knowledge about drugs, drug use, addiction, crime connections, and perceived harms. These myths include: (i) illicit drugs are the most harmful, (ii) cannabis causes death, (iii) recreational use of illicit drugs is impossible, (iv) illicit drug use inevitably leads to addiction, and (v) illicit drug use causes crime. These perceptions are based on social and political constructions rather than scientific evidence.

\section{Myth One: Illicit Drugs Are the Most Harmful}

A great number of survey respondents failed to recognise that many legal drugs like alcohol and tobacco are more harmful than some illicit drugs. As noted in Chapter Four, the present Vietnamese drug classification does not consider alcohol and tobacco as the most harmful drugs, and they are not prohibited under the Vietnamese drug schedules. Nearly $80 \%(n=198)$ of survey respondents did not think that 'some illegal drugs are less harmful than alcohol and tobacco' (see Figure 7.1). 
Figure 7.1: Some illegal drugs are less harmful than alcohol and tobacco

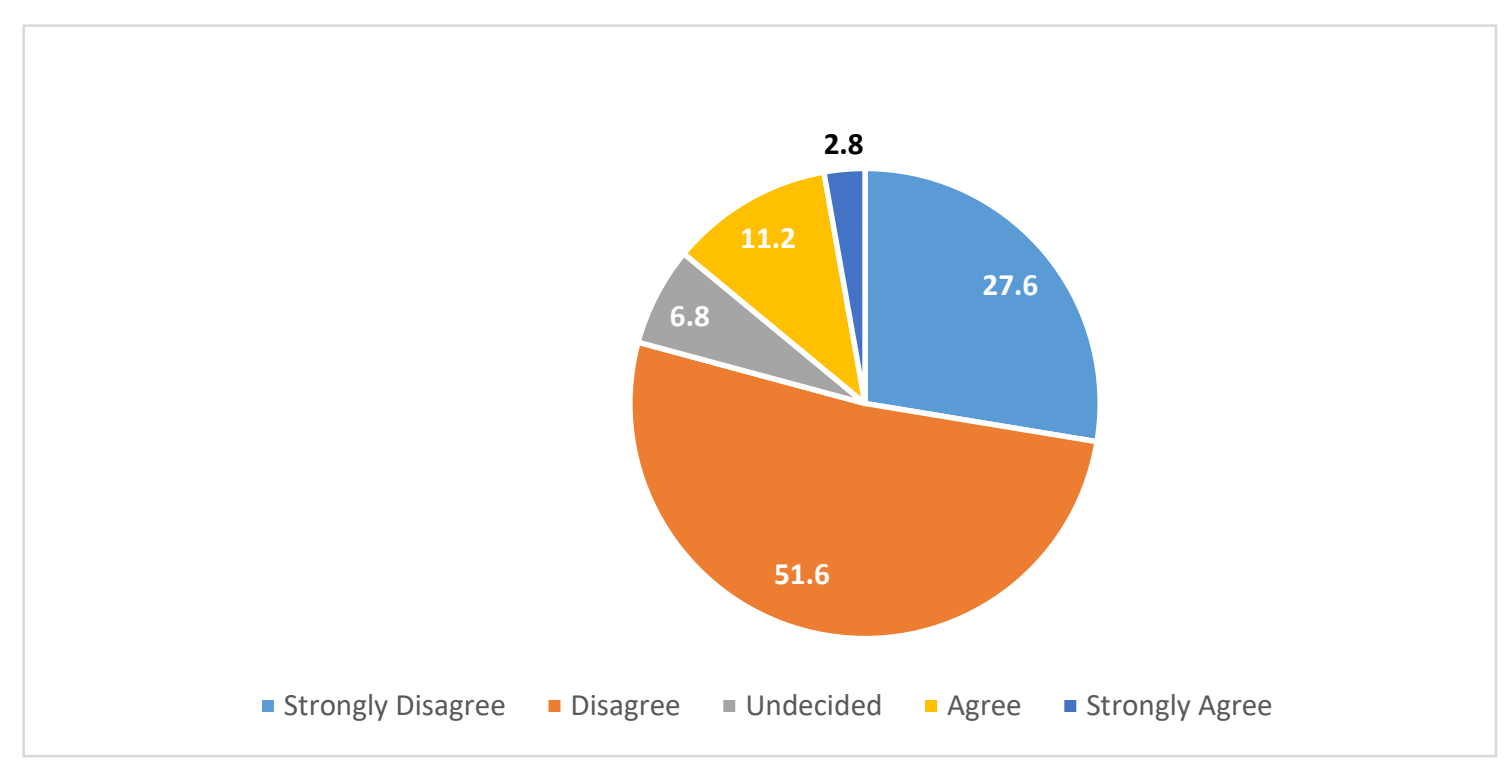

As discussed above, legal drugs (like alcohol and tobacco) are generally acceptable because they contribute a significant amount of tax revenue for the state (Gossop, 2012). Also, they are mistakenly believed to cause less harm than illicit drugs. Meanwhile, illegal drugs are condemned for leading to addiction and health problems (Boland, 2008). In Vietnam, as noted previously, the use of any prohibited drug is portrayed and exacerbated as dangerous, problematic and causing crime, and illegal drug use is propagandised as a social problem that brings many serious threats to users and communities (Vu, 2007; Vuong et al., 2012). Yet as discussed in Chapters Two and Three, these misleading arguments are based on social and political construction rather than evidence. They are widely based on an abstinence approach, and they ignore harm reduction evidence that shows that there is no scientific rationale to support the distinction between illegal and legal drugs (Nutt, 2012).

\section{Myth Two: Cannabis Causes Death}

Another myth is related to cannabis. Less than half of the survey respondents $(n=123)$ did not think 'Cannabis overdose deaths are a problem in Vietnam'. While nearly $30 \%$ of survey participants $(n=74)$ thought cannabis caused overdose deaths, the rest, over $21 \%(n=53)$, had no idea about cannabis related deaths (see Figure 7.2). 
Figure 7.2: Cannabis overdose deaths are a problem

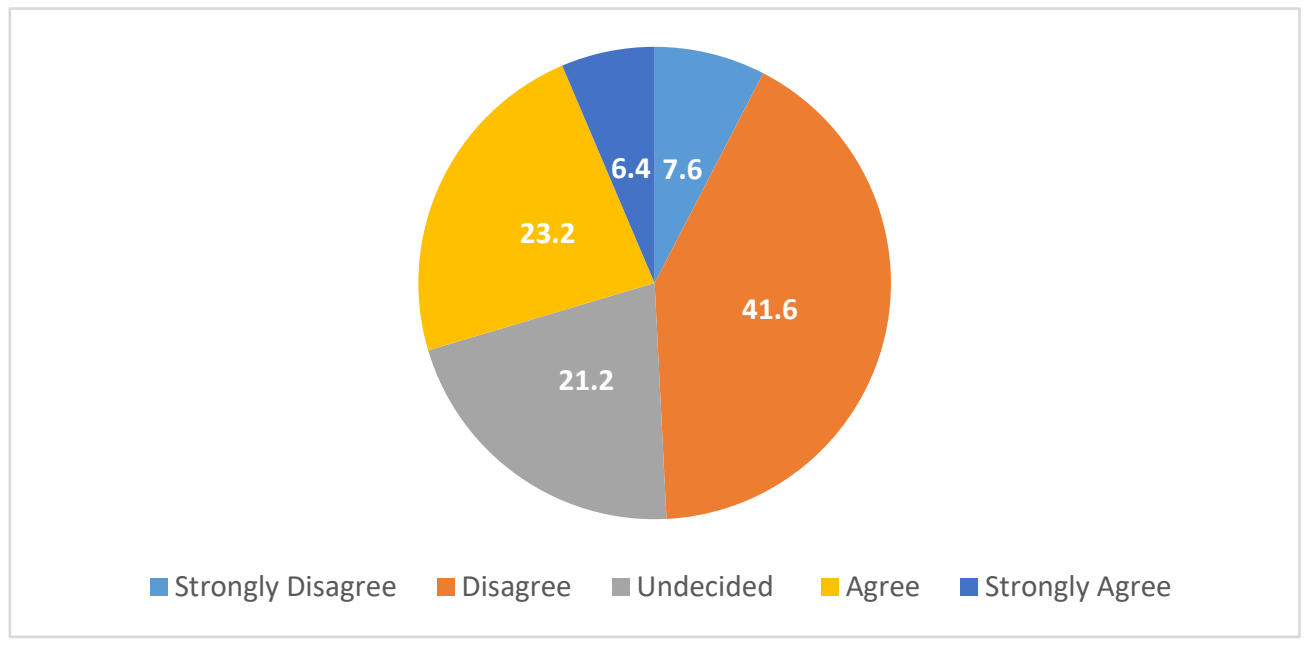

These results generally demonstrate that not many Vietnamese people have sufficient information about cannabis. In fact, in relation to cannabis overdose, there are no recorded deaths so far worldwide (Buchanan, 2016a). This drug has been used in Vietnam for hundreds of years and it is considered one of the most harmful drugs and placed under strict prohibition within Vietnam's drug policy (Vu, 2007). In fact, the Vietnamese government and media have always raised serious concerns about and even exacerbated the danger of cannabis. As noted previously, cannabis is less harmful than legal drugs, such as alcohol and tobacco. The populist views and campaigns about illicit drugs like cannabis is not new. The distorted discourse of cannabis is similar to 'mephedrone', which was discussed in Chapter Two. This dissemination of information about cannabis has perpetuated myths in Vietnam, that are believed by many professionals working in the 'drugs' sector.

\section{Myth Three: Recreational Use of Illicit Drugs Is Impossible}

While a few survey respondents believed that 'Most people who take drugs do so recreationally and rationally', over $85 \%(n=213)$ did not think that people can take drugs in a recreational and rational manner (See Figure 7.3). This common thinking contradicts the scientific evidence presented in Chapters Two and Three (that only a minority of people who use drugs have problems with addiction while the rest take drugs in a controlled way). 
Figure 7.3: Most people who take drugs do so recreationally and rationally

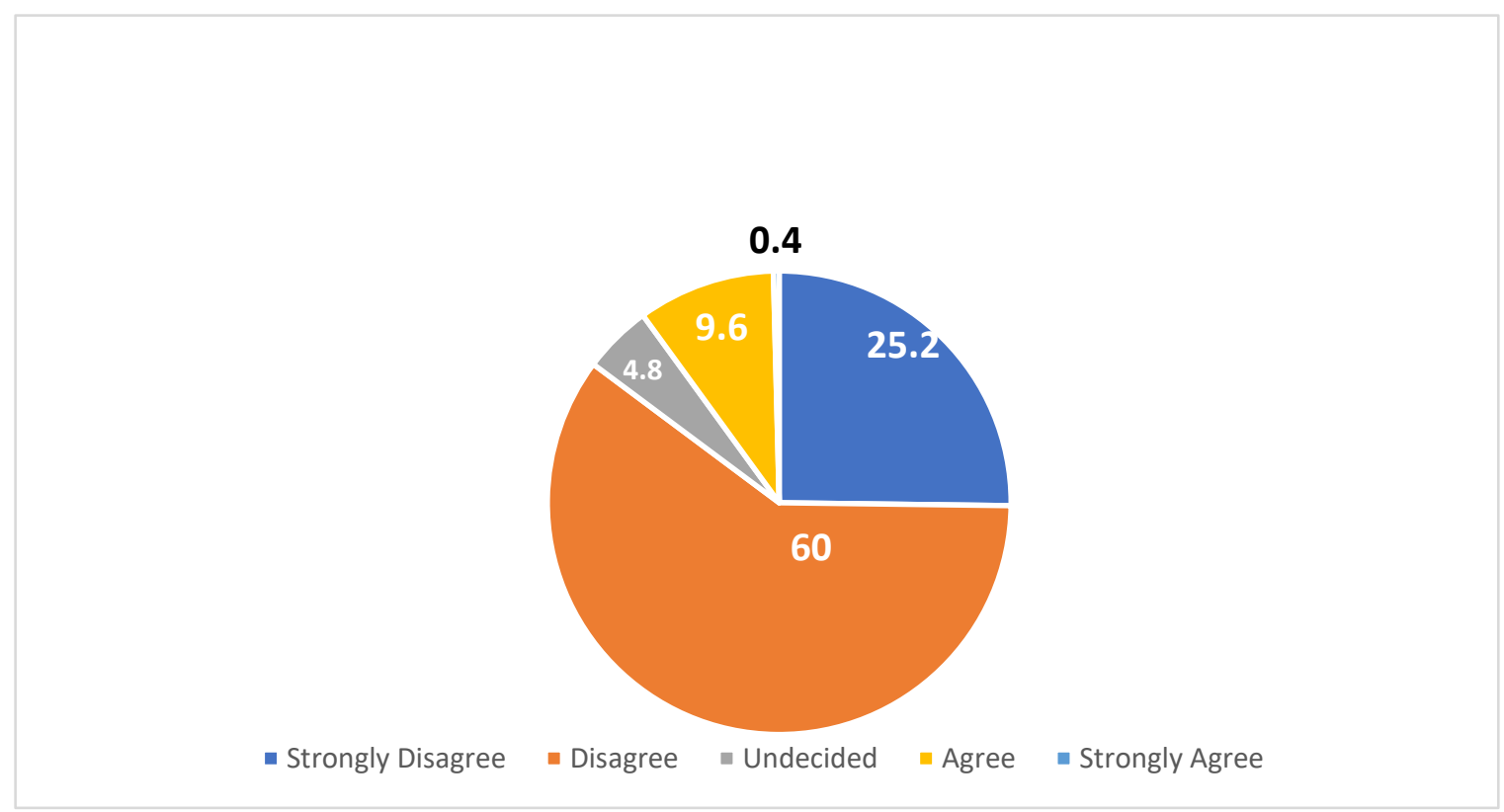

Most interviewees tended to see drug use as being problematic and did not see it from a recreational perspective. Interestingly, one interviewee recognised the myth:

In Vietnam, this (recreational drug use) is not accepted ... I've seen that many people who use drugs who are sent to compulsory treatment or detoxification centres are recreational people who use drugs ... but authorities still think that detaining people who use drugs in the centres is easier to control users than leaving them in the community ... this reflects a common trend in law enforcement agencies, especially in police forces (Government official).

There are reasons to explain why not many interviewees and survey participants thought that people can take drugs recreationally and rationally. First, as discussed in Chapter Four, Vietnamese people often confuse occasional people who use drugs with excessive people who use drugs or 'addicts' (Hong et al., 2011). All people who use drugs are frequently stereotyped for the purpose of highlighting the danger of drug use. Second, this confusion is widespread because official materials and propaganda campaigns frequently make the claim that people 'Use drugs once and you're addicted' (USAID, 2011). This message is widely disseminated across the country in order to encourage people to stay away from drug use. In fact, such confusion over recreational and problematic drug use is globally widespread, not only in Vietnam. These perceptions reflect abstinence-based perspectives and contradict with 
an authentic harm reduction philosophy that demonstrates that only a small number of people who use drugs have an addiction problem while the rest use drugs in a controlled and recreational manner.

\section{Myth Four: Illicit Drug Use Inevitably Leads to Addiction}

The relationship between drug use and addiction also demonstrated confusion among research participants. The majority of interviewees (over two-thirds, $n=17$ ) asserted that drug use will mostly lead to addiction, even though they also perceived differences between recreational or experimental drug use and addiction. In reality, drug use rarely leads to addiction - for example, according to the International Network of People Who Use Drugs (2014) about $10 \%$ of people who use drugs have addiction problems. Yet interviewees portrayed a different view:

They are different, but over $90 \%$ of drug use leads to drug addiction (International worker 2).

Using drugs many times will lead to addiction as an inevitable consequence (Police 1).

Drug use and addiction are quite similar because addiction is initiated by drug use. If people who use drugs can stop using drugs in the first place, addiction can be avoidable. Once they continue to consume drugs, they will be addicted. There is only a narrow gap between drug use and addiction (Psychological Consultant 1).

The conflation between drug use and addiction could explain the rigidity of sticking to abstinence-based models in Vietnam. This conflation is really how drug use and users are viewed, and explain why recreational use is denied and unaccepted. Therefore, it is unsurprising that abstinence-based approaches are popular, rather than authentic harm reduction.

These qualitative findings are consistent with the quantitative results in which eight out of ten survey respondents ( $n=200$ ) 'agreed' or 'strongly agreed' that drug use will lead to drug dependence (see Figure 7.4). 
Figure 7.4: Taking drugs will inevitably cause drug dependence

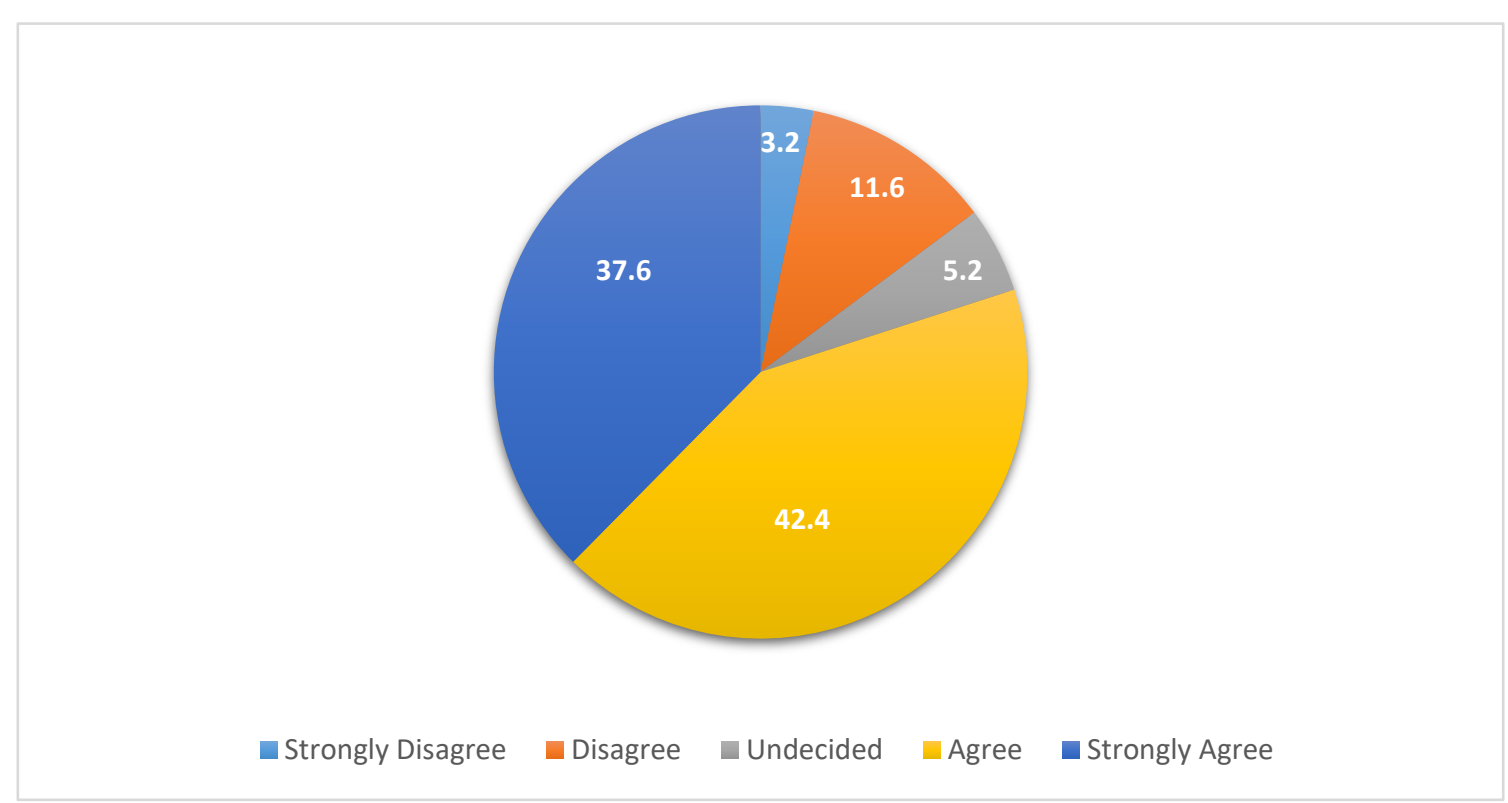

This common perception is expected given the prevalence of Vietnam's media, which shows that illegal drugs are harmful and addictive, and that using illegal drugs will lead to addiction as an 'iron law' (Frey, 1997). As noted above, the Vietnamese media widely uses the motto: 'using a drug even once will cause addiction' (Thien, 2002, p. 39). Also, even the UN argues that drug use leads to addiction (Vuong, 2016), and Vietnam - a member state - is not surprisingly following this model.

Vietnamese professionals tended to think that drug use and addiction are almost always linked. However, as previously shown, in reality roughly about $10 \%$ of people who use drugs struggle with addiction (International Network of People Who Use Drugs, 2014). This misperception has strong impacts on how Vietnam responds to drug use, not least in the existence of 06 centres. These centres treat addiction, so all people who use drugs (even though many are recreational users) are held for addiction treatment purposes (Vuong et al., 2017). They are forcibly 'treated' for a problem that they do not have - and, as noted in Chapter Five, this is counterproductive and creates harm, such as stigma and discrimination, rather than reducing harm for users.

\section{Myth Five: Illicit Drug Use Causes Crime}

Another misperception held by many interview and survey participants is that drug use is a crime problem. Except for interviewees working for public health and international sectors, 
the majority elaborated that drug use is illegal behaviour and it can turn a 'normal' person into an 'offender':

Addiction results in criminal behaviour and social order, having said that addiction causes destructive effects on social life (Police 6).

Drug use is the main reason that triggers crime, social vices, and HIV infection. The negative consequences caused by drug use seriously affect social-economic development and national security (MOLISA official 5).

$50 \%$ of the survey respondents $(n=125)$ regarded drug use as a crime problem (a criminal behaviour) in comparison with $44 \%(n=110)$ of those did not think so (see Figure 7.6).

Figure 7.5: Drug use is a crime problem

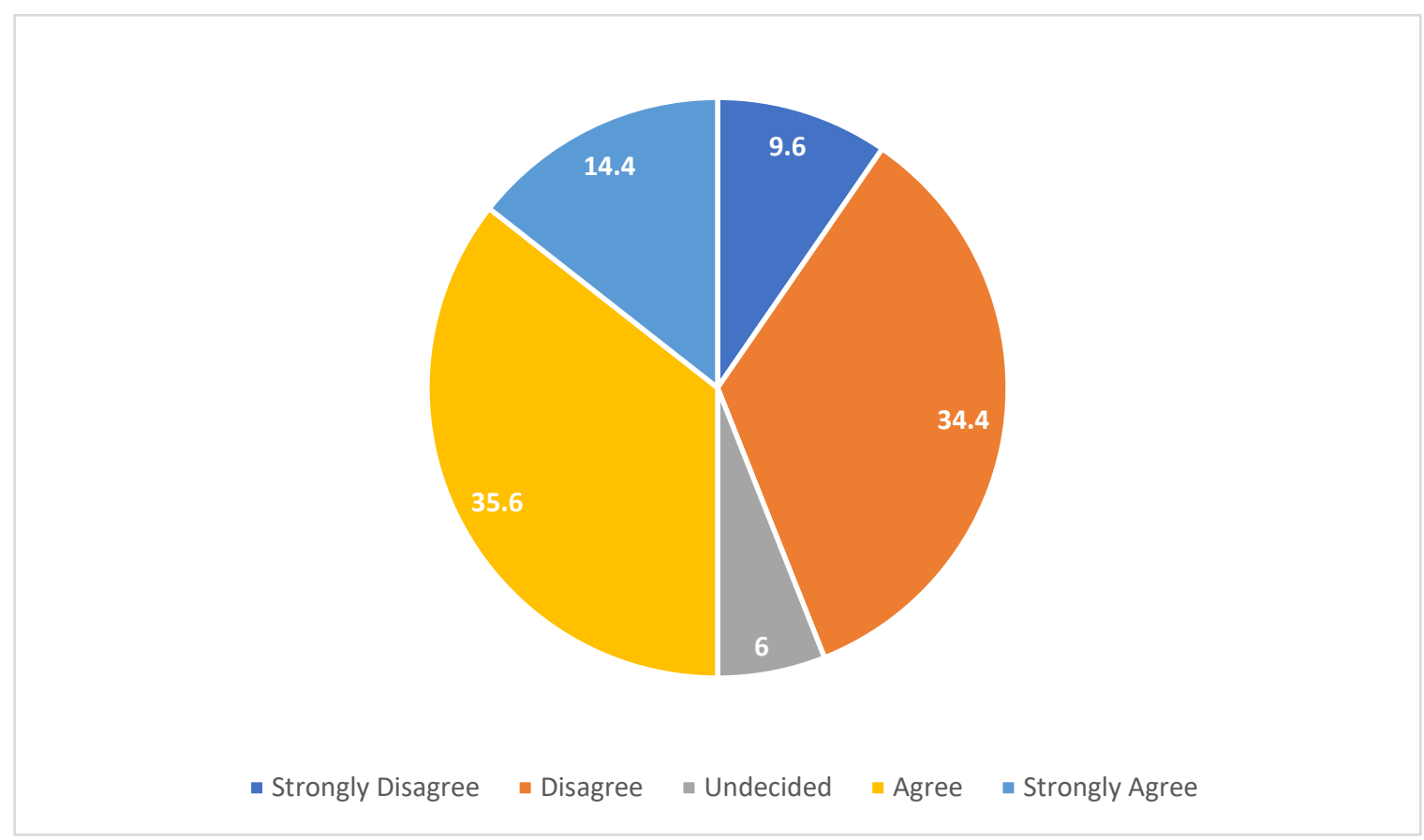

Given the Vietnamese context, it is understandable why many officials still tend to see drug use as a crime problem. They are expected to ensure public security and social order and they largely regard people who use drugs as a problem. Also, the training they have received is mainly based on the political and institutional assumption that drug use is a crime problem. As noted in Chapter Five, it is common that a police officer's performance is assessed on the basis of how many people who use drugs they manage and apprehend. This finding is consistent with the general understanding about drug use in Vietnam (see Chapter Two). 
Many countries and even the United Nations Office on Drugs and Crime also state that there are direct links between drug use and crime (Boland, 2008).

However, as previously argued, the beliefs about the drug and crime causal connections are mainly based on the ideology of the disease and moral model of addiction or moral objections rather than rational science and evidence. People who hold these beliefs try to assume drugscrime connections to control people who use drugs and reasoning for prohibition (Bean, 2014; Buchanan, 2015b). The drugs-crime connection myth partly explains why Vietnamese professionals tend to support the use of 06 centres because, for them, the centres help to reduce crime. However, as noted previously, the connections between drug use and crime are contested and ambiguous (Hammersley, 2008), not least as it generally overlooks the social context in which crime occurs, and denies the role of inequality in generating crimes and in generating the responses to crimes (Stevens et al., 2010). The study findings show that participants ignored underlying social factors, especially inequality or deprivation that result in problematic drug use and crime.

\section{Summary}

Many interview and survey participants held numerous misperceptions about drugs, drug use, addiction and harm. The majority of study participants always perceived illicit drugs as the most harmful drugs, and they tended to ignore the fact that alcohol and tobacco are more dangerous than drugs like cannabis. Cannabis-related information seems to be insufficient, with half of the survey respondents thinking cannabis caused deaths among users. In addition, the majority of research participants did not believe that people can take drugs in a recreational and controlled manner. Accordingly, only a small number of participants could see that only a few people who use drugs struggled with addiction. Another common misunderstanding was that drug use inevitably causes crime. This perception has resulted in the deployment of 06 centres, to detain people who use drugs for the purpose of preventing crime. All these myths demonstrate that abstinence-based mind-sets remain popular among key stakeholders in Vietnam. As a result, although Vietnam has adopted some interventions in the name of harm reduction, abstinence continues to dominate current perceptions of, and responses to, drug use. 


\section{Drug Use}

Perceptions of drug use were multifaceted among survey and interview participants. Largely, drug use was linked to a health deficit, or a public health matter, and often associated with brain abnormalities. Also, drug use was perceived as a social vice, especially among MOLISA workers and police. Further, interviewees highlighted the connection between drug use and personal problems or family dysfunction. The perceptions of interviewees on drug use demonstrate a mix of the moral and brain disease models in Vietnam.

\section{Public Health Issue}

The vast majority of interviewees $(n=20 / 26)$ viewed drug use as a public health issue. As noted previously, viewing drug use as a health matter means that those who take drugs are treated as patients, not criminals. The prevalence of this view is because US models of addiction have strongly influenced Vietnam's drug policy. When the HIV infection emerged as a serious problem in Vietnam in the 1990s, US-sponsored organisations such as WHO, UNAIDS, WB, and UNODC helped Vietnam to implement treatment programmes (Edington \& Bayer, 2013). These organisations widely endorse drug use as a public health issue and, as a result, this has led to the prevalence of a public health perspective when it comes to considering drug use in Vietnam. For example, some interviewees said:

Drug use is a public health issue, not a social evil. It also is a brain disease that needs to be treated (Consultant).

Drug use is a health matter, so it is the reason why the 2009 Penal Code decriminalised drug use behaviours (Police trainer).

Some international workers not only viewed drug use as a public health issue, but they also emphasised its relationship with the human body and brain system:

Drug use is unhealthy behaviour and it affects the nervous system and can damage the immune system (International worker 1).

Drug use is a disease so we need to understand what is [happening] in the brain of people in order to give help (International worker 2). 
The results of the survey also show a tendency to view drug use as a public health issue. Figure 7.8 demonstrates that over $70 \%(n=177)$ of 250 respondents agreed or strongly agreed that 'drug use is a public health issue'.

Figure 7.6: Drug use is a public health issue

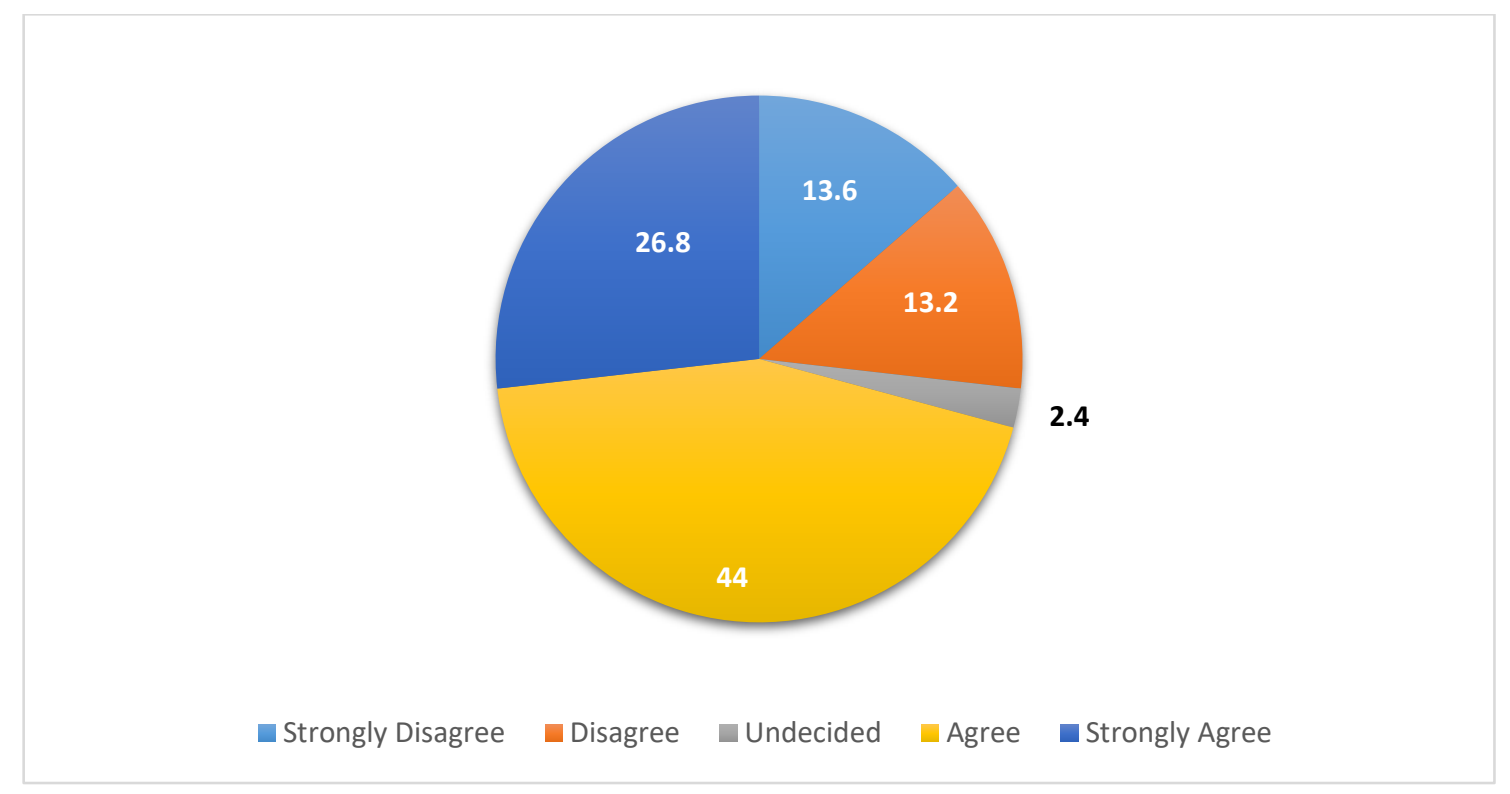

However, it is important to note that, although many interviewees and survey respondents saw drug use as a health issue, which is a positive aspect, the use of illicit drugs is still considered an administrative violation in Vietnam. Accordingly, illicit people who use drugs face the risk of being detained in 06 centres in the name of treatment, and as a public health response. Yet as discussed previously, 06 centres are heavily criticised for having minimal medical services, leading to blood-borne diseases among detainees. Chapter Eight will further discuss 06 centres from study participants' perspectives and explain why the centres are barriers for developing 'authentic' harm reduction in Vietnam.

\section{Social Evil}

Some professionals, especially police and MOLISA officials, tended to see drug use as a social vice that needs to be controlled, administered and, at times, be subject to administrative sanctions. Their perspectives are understandable given their role ${ }^{71}$ in controlling and punishing drug use:

\footnotetext{
${ }^{71}$ As noted in Chapter Five, MOLISA officials organise and manage people who use drugs in drug treatment centres and communities, while the police are charged with formulating
} 
In my view, taking drugs is a social-psychological matter ... it also is a social evil or a sort of social vice (Police 1).

Drug use is a social and psychological problem, but from another perspective, drug use is illegal behaviour that can be punished by the administrative law (MOLISA official 1).

Their opinions about drug use are contradictory here. They deemed drug use as a social and psychological matter, but also consider it as a social evil. As noted previously, police and MOLISA officials are trained for and charged with preventing social evils, including drug use, in Vietnam. Therefore, it is unsurprising that they perceived the use of drugs as a social evil behaviour.

\section{Social and Psychological Perspectives}

Almost all interviewees highlighted social and psychological factors to explain why people take drugs. The most common reasons for drug use were that users were 'personally showing off', 'looking for fun', lured by friends or 'easing pains/depression'. Interviewees had a tendency to link these reasons with young people who were seen to be lured to drug use. To illustrate why people take drugs, especially young people, some interviewees explained:

Many people take drugs because they are enticed and lured, they want to show off their personality. Drug use is mainly a social tendency among young generations (Police 2).

Many young people tried drugs because of curiosity not to mention many took drugs just to forget problems related to their love and their life (International worker 3 ).

In addition to social-psychological factors, many interviewees mentioned family factors. They argued that the insufficient attention of family to young generations is a key factor in drug use:

A lack of attention and education of family, parents overloaded with their work, so they seem to not care for their children. Once parents don't care for their children

policies and strategies for 'fighting' drug use, drugs crimes and in helping MOLISA to ensure the security of compulsory treatment centres. 
enough, children will look for other alternatives to handle their issues ... if they join in with a 'bad' friend group that is using drugs, they are easily lured into drug use (Harm reduction professional 1 )

Family, they tend to blame those working in education and others, but have they ever asked themselves if they have enough care and attention for their children or not? ... parents leave home around 7 or $8 \mathrm{am}$ and just back home around 7 or $8 \mathrm{pm}$... children lean on maids and the parents don't know what their children do ... the care of children in the family is a great alarm (Governmental official 1).

In Vietnamese culture, family has an important role. As noted in Chapter Five, the 'Gia dinh van hoa'72 movement is used to assess a family's reputation. Also, Confucianism, which highlights the importance of family, has a strong impact on Vietnamese culture and customs. As Hayes-Larson et al. (2013, p. 2) clarify:

Vietnam is heavily influenced by Confucianism, which holds that the self should be considered last and that one should always devote one's attention to the country and the family first. Communism, which was introduced into the country in the midst of harsh wars, echoed this norm in order to unify the country and mobilize people.

In fact, the word 'family' was mentioned 612 times by 26 interviewees (Nvivo's 'Word Frequencies'). Therefore, it is clear that the family factor is not only important in relation to perceptions of drug use, but also to how users are treated. In Vietnam, families are acknowledged as backbones of society, and they are argued to have strong influences on why some people use illicit drugs while others do not. For example, Vietnamese researchers, like Nguyen and Scannapieco (2008) or Duc (2012) highlight that the lack of care and attention from parents and family members is one of the main reasons people take drugs. Yet as discussed previously, there are many social and psychological reasons for taking drugs and considering the 'family factor' is only the tip of the iceberg.

72 A major social movement in Vietnam, which sets up many requirements (parents and children are harmonious, have good business, have good relationships with others, no law violations, etc.) to be a 'good' family. A 'gia dinh van hoa' needs to meet such criteria. No family members involved in drug use is a key criterion. 


\section{Personal Factors}

Interviewees also often mentioned personal failings as a reason for drug use. Most viewed that those who take drugs do so because they are weak, have psychological problems or lack the determination to steer clear of drugs. For this reason, interviewees tended to view personal factors like peer pressure as a leading reason for drug use:

In fact, there are many good young persons, but they still use drugs ... that is why I said it depends on their determination. Their friends have strong influences on them, if they are not brave to deny temptations, they easily slide into drug use (Doctor 1 ).

In the same social environment, if people are strong and persistent, they will not use drugs ... but some people are not ... this is the key for people who use drugs (MOLISA official 2).

These opinions link to the conflation of drug use with addiction and that all drug use leads to addiction, as discussed in Chapters Two and Four. Vietnam does not really consider recreational drug use, and it sees all drug use as problematic. Also, blaming personal factors for drug use is associated with a moral model of addiction. This explains why Vietnamese drug policy mainly focuses on people who use drugs rather than addressing social, psychological, legal or economic disadvantages affecting them.

Similar findings emerged from the survey, as precisely two-thirds of respondents $(n=165)$ did not see drug use as a normal human behaviour (see Figure 7.9). This finding generally reflects a moral model of addiction. 
Figure 7.7: Drug use is a normal human behaviour

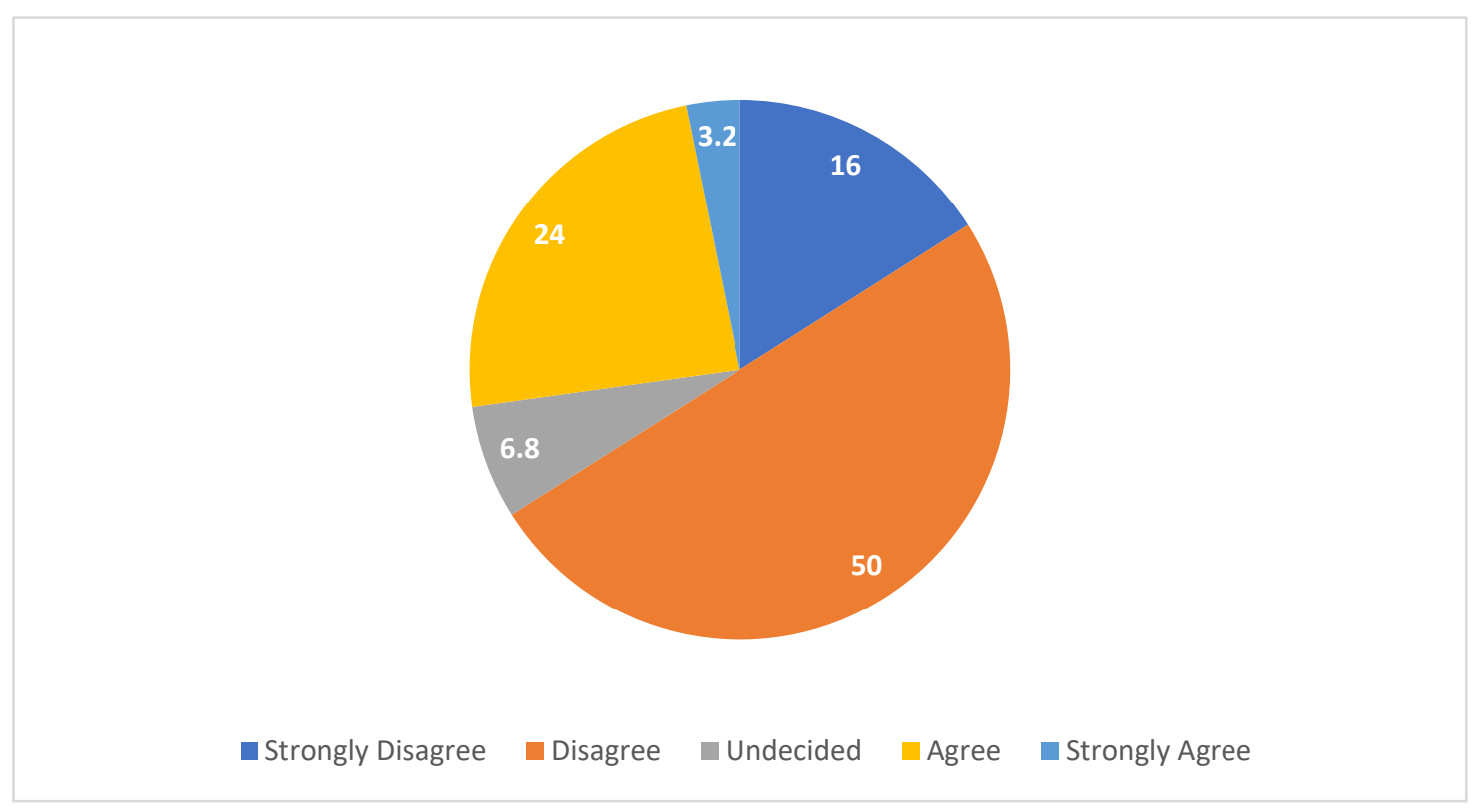

In summary, interviewees and survey participants held multifaceted and contradictory perceptions of drug use. The majority viewed drug use a public health matter while some, especially police and MOLISA professionals, regarded it as social vice. Regarding the reasons for drug use behaviours, interviewees emphasised social, family and personal factors. These results are expected given Vietnam's culture, which emphasises the importance of family and personal responsibilities in society. In going against social norms and conventions, people who use drugs face punitive responses (discussed further in the following chapter). These perceptions of drug use generally reflect a combination of the moral and brain disease models of addiction that underpin the widespread abstinence approach in Vietnam.

\section{Addiction}

While the vast majority of interviewees and survey respondents regarded drug use as a public health issue, over two-thirds of interview participants defined addiction as a chronic brain disease that needs long-term treatment. The brain disease model of addiction is widely accepted by the participants working for all agencies, including Vietnamese and international workers. For example, some interviewees articulated:

Addiction is a chronic brain disease that is hard to treat, and it is easy to relapse. The more time users consume drugs the higher their level of addiction will become (Doctor 2). 
Addiction is associated with public health issues, and it is a chronic brain disease. This disease, therefore, needs long-term treatment to recover brain function (Harm reduction official 2).

Addiction is, of course, a health issue or it is a brain disease. When people use drugs their brain will be affected, leading to changes in the metabolic process. Therefore, the brain needs such drugs in order to help users to feel normal (International worker 4).

The survey also helped to explain the prevalence of the brain disease model, which requires complete abstention from drug use. When being asked whether 'People who use drugs need to quit all drugs and stay drug-free', over $70 \%(n=176)$ of survey respondents answered in support (see Figure 7.10).

Figure 7.8: People who use drugs need to quit all drugs and stay drug-free

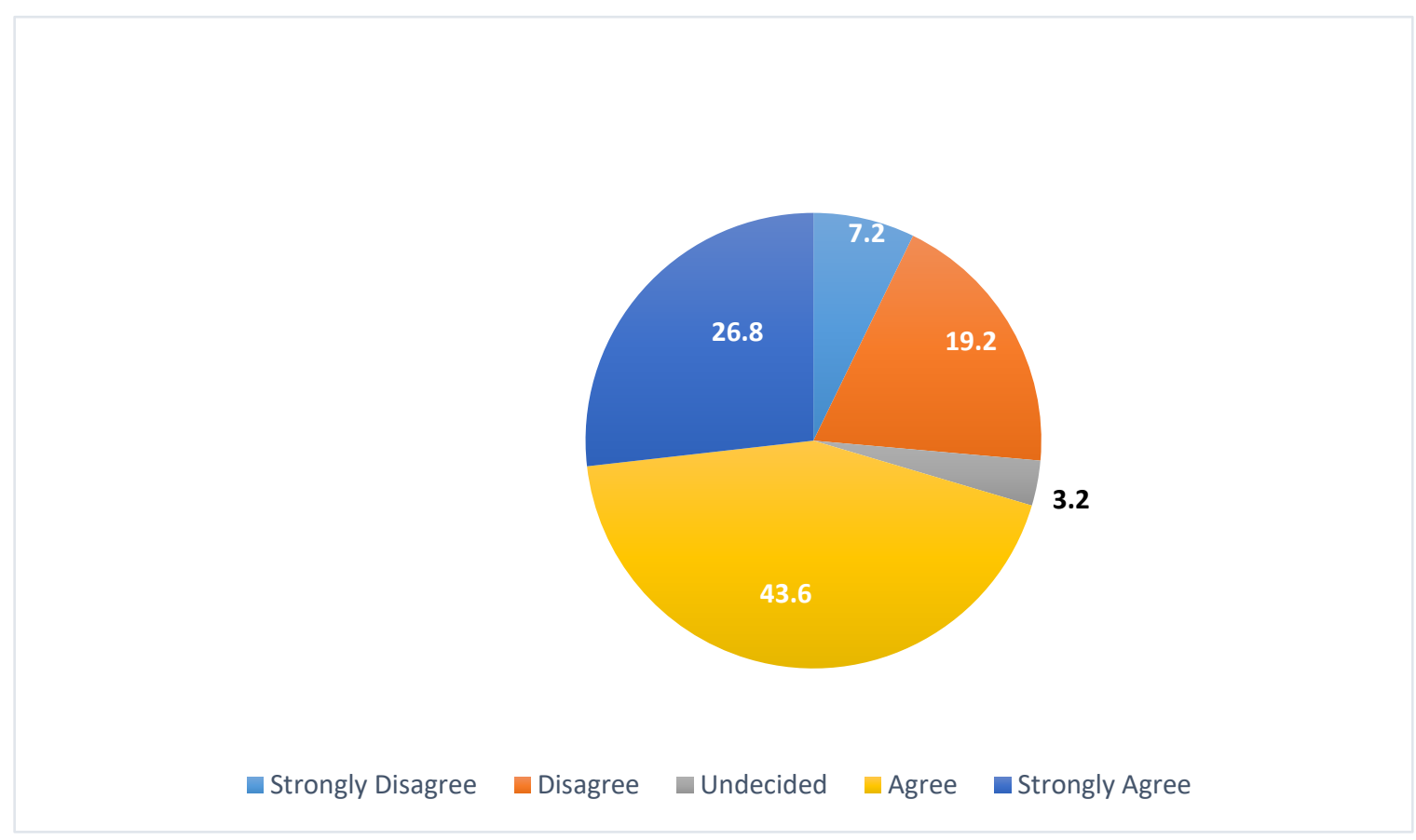

Regarding the brain disease model, a few interviewees viewed that a person's genetic makeup was an important element in addiction. For them, people who use drugs are more easily addicted to drugs than others because of their genes. They also believed that some users became addicted the first time they tried drugs: 
Biologically, many people have a gene called 'an addictive gene'. This does not mean users will be addicted immediately, instead, for someone, their drug intake is higher than others. Some persons get addicted after trying the first time, others take drugs up to ten times, but they are not addicted. This is because their genes are different (MOLISA official 2).

Genetic factors are important in causing addiction. Over $40 \%$ of addicts have a gene that makes addicts lose their balance and easier to have addiction (Police 3).

Yet considering genetic factors for causing addiction is really contested. As Hart (2017) argues, it is almost impossible to distinguish the brains of addicted persons from others, and genetic information is never enough to understand drug use and addiction. Instead, he deems social factors to be more important. His arguments are similar to social and psychological models of addiction discussed in Chapter Two.

Notably, some participants pointed out that addiction is not only a brain disease, or a biological disease, but it is also a psycho-social problem. For instance, they reflected:

Addiction is not only a biological disease or a disorder of the brain, as it is different from other normal medical diseases. It is significantly affected by psychological and social factors (Social worker trainer).

Drug addiction is a bio-psycho-social problem. There's the biology in a sense that the individual might have craving and desire for the substance and when the substance isn't in their system they feel sick or get sick. There may be psychological issues associated with it and they turn to the drug or to the substance as a means of dealing with issues and problems in their life. There's a social function to it as well, in the sense that an individual who's addicted (and, meaning, when I say that, that their focus is $100 \%$ on gaining and using their drug) that means their family and social relationships are going to suffer or be impacted, and so there's a social component (International worker 1).

Acknowledging the social and psychological components of addiction was not so common among interviewees because, as noted previously, Vietnam views addiction as moral and brain disease models of addiction. The media and government frequently propagandise drug 
addiction as a social evil, and this explains why abstinence-based approaches (punishment and incarceration) are widespread. Holding negative perceptions of addiction, it is unsurprising that authentic harm reduction, which is based on a non-judgemental approach, is still difficult to integrate into Vietnamese drug policy.

In addition, over half of the study interviewees identified 'relapse' as an important element of addiction. They acknowledged however that relapse is a normal and natural phenomenon. As noted above, Vietnam like many other Asian countries has high relapse rates, ranging from $70 \%$ to $90 \%$ (MOLISA, 2015). Therefore, interviewees argued that relapse should not be seen as a personal failure or a moral failing. Some explained:

Relapse is normal behaviour because addiction is chronic. This is, therefore, a natural phenomenon of patients addicted to drugs (Harm reduction official 3).

It's a chronic relapsing disorder. So relapse shouldn't be seen as a failure of the individual's moral character, it's basically a component of the disease ... it is part of the disease, and I don't think it's a moral failing (International official 1).

Their progressive views are significant given the prevalence of brain disease and moral models of addiction in Vietnam. Unfortunately, these views came from a few harm reduction workers and international officers, while the majority still viewed relapse as a personal failure.

For example, many interviewees placed the blame on people who use drugs for their problems or their failure to quit drugs.

Society and government have provided drug addicts with plenty of support, they should blame themselves for their failures to quit drugs. We gave them opportunities but they did not take it (Police 2).

The most important factor is people who use drugs themselves ... because we have backed them up for too long, their families and society have been behind them ... but they just seek their own interests instead of demonstrating their responsibilities to society and their families (Doctor 3 ). 
These perceptions clearly show the dominance of punitive and user-blaming approaches, which is linked with the moral model of addiction.

Further, although no interviewees clearly defined addiction as a moral issue, the way they perceived drug use, and people who use drugs generally demonstrated the prevalence of the moral model of addiction in Vietnam. For example, one interviewee said:

Addiction means that users cannot live and normally function without taking drugs, they take drugs without caring about the consequences, and they are ready to abandon daily pleasures, their families, their friends, and their work to use drugs. They see taking drugs as a hobby, the greatest pleasure in their life (Doctor 4).

As can be seen, although the majority of interviewees viewed relapse as a normal and expected issue, they tended to use personal factors to explain relapse. This is not unusual given the moral model is still widespread in Vietnam. Interestingly, although nearly all interviewees deemed that relapse is normal, many survey respondents (over $78 \%, n=177$ ) did not consider that 'drug relapse should be viewed as a learning experience' ${ }^{73}$ (see Figure 7.11).

73 In treatment, relapse is acceptable and it should be seen as a natural and expected occurrence (MacMaster, 2004; Marlatt, 2000). In other words, relapse is an expected step that those who are addicted to drugs will experience during the process of overcoming their addiction. Also, if people who use drugs continue to use drugs after treatment, this should not be viewed as an individual's failure or lack of ability, but instead, should be seen as a learning experience (MacMaster, 2004). 
Figure 7.9: Drug relapse should be viewed as a learning experience

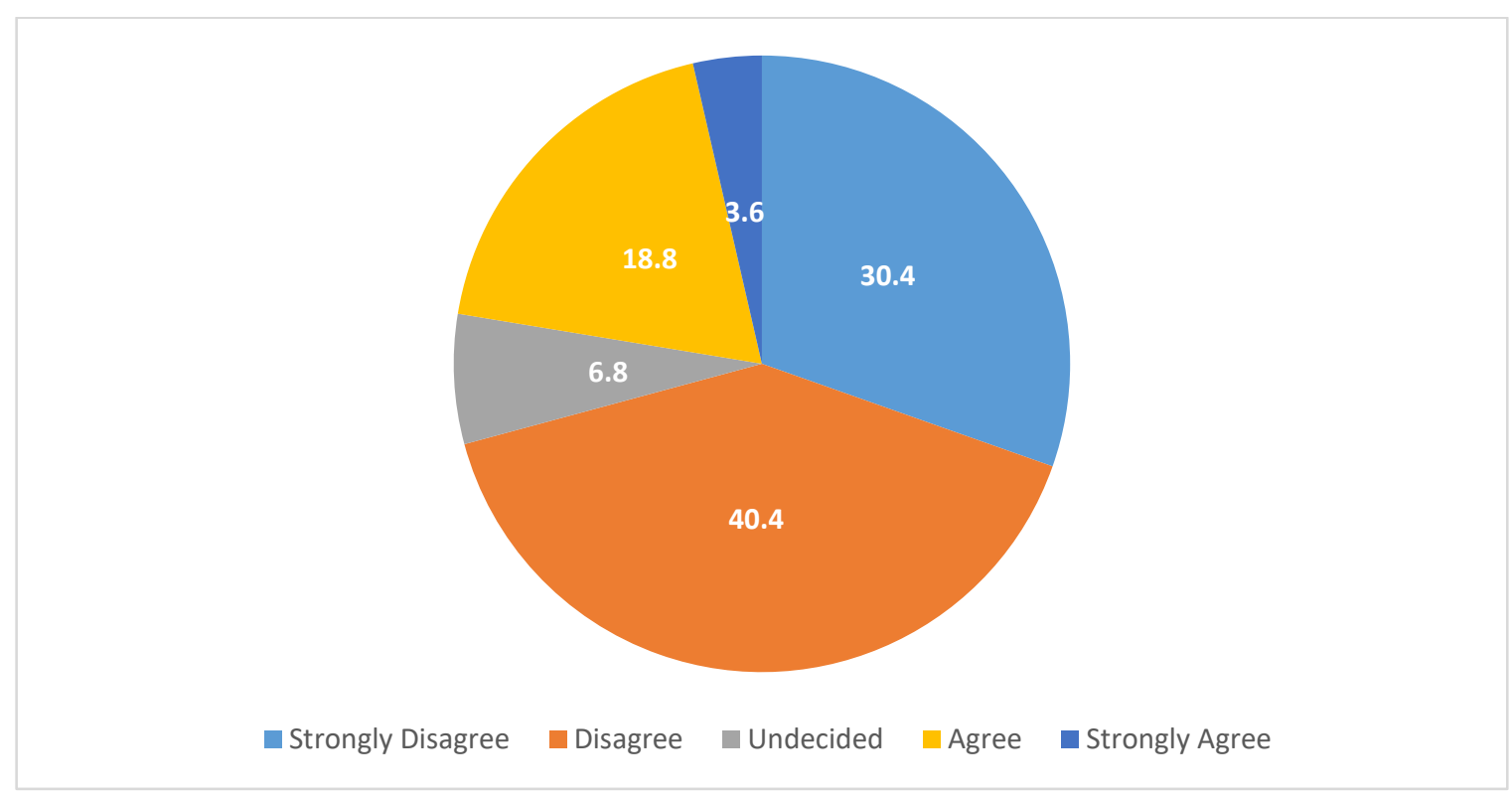

This result can be explained by the fact that, as noted in the previous section, Vietnamese people tend to stress the importance of personal factors to explain why people take drugs and become addicted to drugs. Vietnamese people often view that those taking drugs are weak, have psychological problems or lack determination to steer clear of drugs. As a result, people who use drugs are widely blamed for their relapse. This common thinking has led to an emphasis on individualised medical solutions and moral rehabilitation to prevent relapse and to reform people who use drugs, generally ignoring the complexities of drug use behaviours and the intersections between drug use and social or environmental factors.

Close to a social and psychological model of addiction, many informants emphasised that unemployment, lack of education and social support, stigma or discrimination are underlying reasons that force people who use drugs to relapse or that worsen their addiction. Interviewees articulated:

Social factors play an important role in shaping addiction and addictive situations. When addicts return to the community, those factors will affect them and can lead them to relapse. If we don't have supports, those factors can exacerbate an addict's addictive situation. As I said before, without providing them with psychological consultation and employment support or social services, addicts will easily collapse and use drugs again (Social worker trainer 1). 
Not using alcohol and drugs is just one measure. Having a job, having a family, having good social relationships; I used to have a boss who would say 'Look, the best thing a person wants to do is not use drugs, have a job' (International official 1).

Addicts try their best to re-enter and re-integrate into society, but when they apply for a job, they face social stigma and discrimination, not only one time, so this generally forces them back to addiction (Harm reduction official 3).

These views are unpopular within the abstinence orientated environment in Vietnam, but they are essential to develop authentic harm reduction. As noted earlier, Vietnamese interventions mainly aim to prevent drug use and stop people from using drugs, while social, economic or legal issues around people who use drugs are ignored. As always, protecting society is the first priority of the government, not the benefits of people who use drugs.

In general, addiction was widely considered a brain disease among interviewees and survey respondents. The majority of interviewees failed to acknowledge the importance of the resources, support networks, personal agency, and social and cultural capital in considering why people become addicted. Instead, those who take drugs or are addicted to drugs were perceived unfavourably by many interviewees and survey participants. However, many other interviewees were inclined to underline other social and psychological factors as the crucial components that have strong impacts on how and why people become addicted and relapse occurs. In other words, while, philosophically, most research participants perceived addiction as a brain disease, some entwined it with psychological and social elements, especially with regard to relapse. These results among interviewees are promising as they reflect a more nuanced understanding of drug use. Yet the majority of survey respondents did not view relapse as a learning experience and they blamed personal factors for drug use and addiction. Those perceptions of addiction again reinforce the prevalence of abstinence-based thinking among Vietnamese stakeholders, even though Vietnam has adopted some interventions in the name of harm reduction since the 2000s. 


\section{People who use drugs}

Given the dominance of the public health model, most interviewees, except some Police and MOLISA officers, tended to see those who take drugs as patients, who have a disease associated with the human brain system:

We see addicts as patients, and they suffer a special disease related to a disorder of the brain system (MOLISA official 1).

People who use drugs are normal persons, but when they get addicted they become patients (Harm reduction officials 4).

Similarly, a large proportion $(61.6 \%, n=154)$ of survey respondents were inclined to think that people who use drugs are suffering a disease (see Figure 7.12).

Figure 7.10: Drug users are suffering from a disease

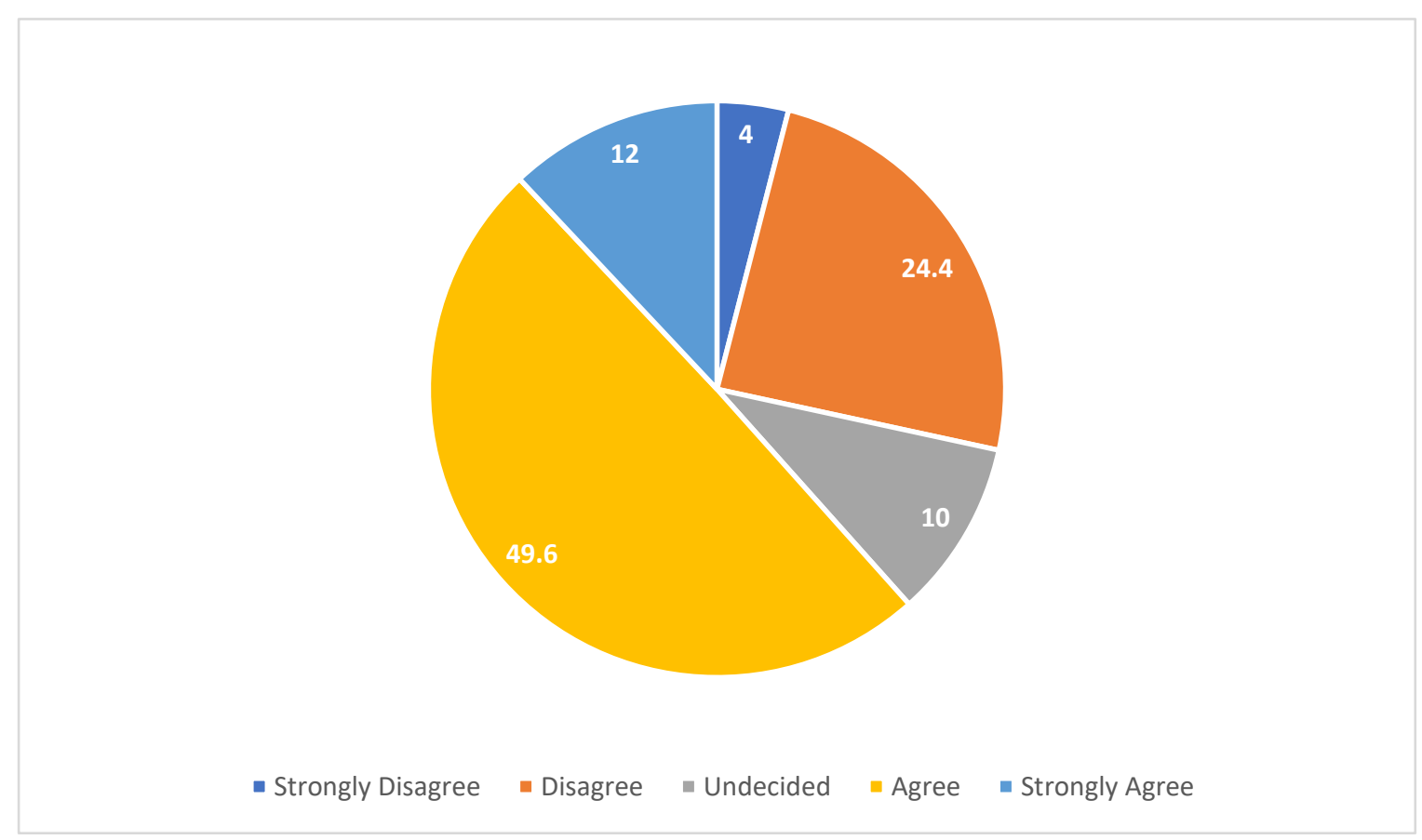

Although people who use drugs were regarded as patients on paper, many interviewees still had a negative attitude towards them. The Vietnamese government and media tend to portray people who use drugs as 'bad people' who have low moral standards and who violate Vietnamese social conventions (Hong et al., 2011). This generally explains why many 
interviewees did not like people who use drugs and viewed them as a high risk group who had moral problems and who were responsible for many anti-social and criminal behaviours.

I have to admit that in our society, no one likes people who use drugs. Good people, non-people who use drugs, those who wish to have a peaceful life, none of them like people who use drugs (Government official 1).

Vietnamese society tends to believe that only bad persons take drugs, those taking drugs mean they are bad ones ... this notion is deeply ingrained in most people (International worker 5).

Some interviewees emphasised the media's role in shaping general attitudes toward people who use drugs. Negative images of people who use drugs are widely presented, and users are frequently depicted as causing social problems. Their representation, as being against social conventions and ethics, provokes 'society distaste' for who they are:

It cannot be denied that information from media significantly affects official responses related to drug use problems. And I believe that what the media represents is right about people who use drugs and the drug use situation. They do not exaggerate, drug use is a potential risk to society (Police 1).

The media in Vietnam are diverse, including television, radio, the Internet ... but most of the contents emphasise deterrence, controlling, and defaming people who use drugs rather than support and respect (MOLISA official 3).

Nevertheless, some international workers revealed that the media provides or disseminates misinformation:

Vietnamese media tend to show bad images of addicts, with addicts not being able to escape from their addiction. Now, people propagandise addiction as a disease and drug addicts as patients, but discrimination and stigma are still common (Social worker trainer).

Regarding media, to be honest, what they show is fiction (International worker 5). 
In television programmes, they'll drop the addict or the recovering person talking about their situation. They'll drop the performance by individuals who are suffering from the disease if time is short. So it's there, and it's given a space on the agenda, but if they can chop it off they'll chop it off (International worker 1).

In Vietnam, people who use drugs are viewed as social evils and so that is why they are treated like outsiders. As noted previously, the government and media often use negative caricatures to represent people who use drugs, reinforcing the idea that people who use drugs are bad people who make bad life choices (Nguyen \& Scannapieco, 2008).

In summary, although drug use is seen as a public health issue on paper and people who use drugs can be treated as patients, the prevalence of official campaigns against social evils (including drug use) has stigmatised people who use drugs, and isolated them from their families and communities. The dominance of abstinence-based approaches in Vietnam has led people who use drugs to be seen as potential criminals. Under a framework of 'moral populism' (as discussed previously), the government often categorises people who use drugs as dangerous, morally reprehensible and diseased persons who are in need of harsh punishment and control. Given the prevalence of negative perceptions towards people who use drugs, it is difficult to implement authentic harm reduction, which is based in a nonjudgemental approach. Instead, reducing harm in this context (Vietnam) equates to reducing and controlling people who use drugs, and promoting abstinence over harm reduction.

\section{Harm}

\section{Drugs and People who use drugs as the Key Harms}

Based on the findings above, it becomes obvious that drugs and people who use drugs were identified as the key harms. Most interview participants only believed that the use of drugs has destructive impacts on users, their families and society and they did not mention any positive implications of drug use. Some interviewees explained that drugs contain addictive and detrimental substances that will damage users' brains and alter their behaviour in a negative way. Other harms mentioned were that drugs lead to economic burdens on users and their families and that drugs cause HIV infection, hepatitis $C$ and other blood-borne diseases, and even criminality: 
I see that ice drug and heroin use cause financial problems to users and their families, and lead users to criminal behaviour because money spent on buying drugs is huge (Doctor 2).

Drugs cause blood-borne diseases like HIV infection, hepatitis $C$, and taking drugs also lead to unsafe sex ... those who take drugs may commit crimes such as theft, murder, or affect social order and social security ... these are the aftermaths of using drugs (MOLISA official 3).

While drugs were seen as a harm to society and the community, many participants, therefore, claimed that those who take drugs are also potential harms. People who use drugs were perceived as dangerous individuals who cause chaos in the wider community. To illustrate why people who use drugs induce harm, some interviewees explained:

The biggest groups are drug addicts and those who committed drug crime ... the motivation and purposes of the groups are to lure more and more new addicts, and then they are going to have new 'addicted friends' taking drugs together (MOLISA official 1).

State agencies claim that drug addicts are social evils, have moral problems ... the common stereotypes about them are very adverse ... for all citizens, seeing addicts is an awful experience (Governmental official 2).

As discussed in Chapter Four, Vietnamese authorities tend to frame harm within a spectrum (in which harms affect individuals, community and society) and these harms are caused by drugs and drug use itself. Harm is derived from the ravages of drugs and their inherent addictive properties, which lead to a brain disease (addiction) and danger. The perceptions of these harms continue to demonstrate the prevalence of abstinence-based mind-sets among Vietnamese stakeholders, which have strongly influenced how they respond to drug use and harm within a Vietnamese context. 


\section{Wider Harm Perspectives}

Although the vast majority of interviewees regarded that the harm of drug use has been caused by drugs and people who use drugs, some interview participants viewed the harm from wider perspectives. They consider harm from social, environmental or legal perspectives, as highlighted in Chapter Three. For example, one international informant described:

There are seven areas in which an individual's life which can be affected by drug use or addiction or dependence. Obviously, medical issues can occur, employment issues can occur, education issues can occur, legal issues definitely can occur, family and social issues can occur, and psychological or emotional issues can occur. In terms of medical, some common things around people who inject drugs are Hepatitis C, HIV. In terms of other drugs, again it can still be HIV, in the sense that people who drink a lot and people who maybe use ice might have multiple sex partners, might not use condoms, and might get HIV in that regard or other sexually transmitted diseases (International worker 1).

This interviewee also pointed out other harms caused by legal drugs that are often ignored in Vietnam:

The principal problem is alcohol because everybody in this country - it's a drinking culture, and binge drinking ... And tobacco is the next drug that's a major problem in Vietnam, although rampant alcohol scene is a social beverage and tobacco is seen just as, again, as a social thing although there are changes on the tobacco side in Vietnam ... although it's not counted, there are a lot of motorbike accidents where drunk drivers or other people have been drinking at lunch and they have an accident on the way home, or they go to beer 'Hoi' and get drunk and they still get on their motorbike and drive (International official 1). 
Another participant who works for a harm reduction office also acknowledged that other drugs like alcohol and tobacco brought the most dangers, having high rates of morbidity and mortality, but the country seems to 'overlook' ${ }^{\prime 74}$ this fact. They stated:

In Vietnam, alcohol and tobacco addiction are the most serious problems, they cause many harms like domestic violence or traffic accidents ... those harms are even more dangerous than heroin (Harm reduction official 2).

Although alcohol (Ruoi) and tobacco (Thuoc La) are seen as harmful, they are socially and culturally adopted as normal. One interviewee articulated this issue:

My understanding of Vietnamese, you don't have a word 'drug' or even 'substance'. You either have 'Thuoc' or medicine, good things, or you have 'Ma Tuy', the bad things. And I think in part it's language because you don't have a way of talking about them generally. You even don't talk about, because even the legal drugs can be used illegally but you still don't call them 'Ma Tuy' (International 2).

Although a few interview participants could see harm from a wider perspective, no one reflected on the harms caused by the societal isolation and marginalisation of people who use drugs, or the unintended consequences of prohibitive drug policies. As Taylor et al. (2016) argue, prohibition remains the primary source of harm for illicit and people who inject drugs. This is widespread in many countries, not only in Vietnam, but none of the interviewees discussed about the side-effects of prohibition. As detailed above, the harms that were conceptualised by interviewees are mainly caused by drug use and people who use drugs, and these harms include health-related problems, addiction and crime. Therefore, it can be expected that reducing harm from Vietnamese perspectives means eradicating drug use and reducing the number of people who use drugs or controlling them.

\section{Conclusion}

The misleading understandings about drug use, addiction and people who use drugs have led to numerous mythical perspectives among interview and survey participants. The dangers of

\footnotetext{
${ }^{74}$ One of the reasons to explain the ignorance is due to language, such as the use of the stigmatising 'Ma Tuy' to denote illicit drugs (as discussed previously).
} 
alcohol and tobacco were overlooked while the knowledge related to cannabis was limited. Notably, only a few informants believed that people can take drugs recreationally and rationally, and the majority thought that all people who use drugs have to struggle with addiction. The most common myth was that drug use causes crime. This perception has resulted in the prevalence of 06 centres where the government detains people who use drugs with the aim of preventing crime. These common myths have strong impacts on how Vietnamese define the harms caused by drug use. Apparently, perceived harms were derived from drugs, drug use and people who use drugs, and no interviewees mentioned harm caused by the current drug policy, which results in significant harm, as discussed previously. Therefore, reducing harm from Vietnamese perspectives means reducing drug use and controlling people who use drugs as it does in other countries.

Drug use was regarded as a public health issue by the majority of survey and interview participants, while a few interviewees, mainly working for MOLISA and MPS, had a tendency to see drug use as a social problem or social evil. This common thinking among MOLISA and MPS professionals is understandable, given their role in addressing drug use in Vietnam. The reasons for drug use are explained from many perspectives, but interviewees tended to emphasise the role of individuals and family, and some highlighted social factors. The personal and family factors are generally associated with Vietnamese culture, which is heavily influenced by Confucianism. The common perceptions of drug use led to the fact that a great number of interviewees considered addiction from a brain disease perspective.

Nevertheless, many interview participants underlined social and psychological factors as the crucial components that have strong impacts on how and why people become addicted and relapse occurs. The personal factors were stressed as the key component of addiction. Many interviewees thought that those addicted to drugs are weak, have psychological problems or lack the determination to steer clear of drugs. This common thinking generally reflects the ideology of the moral model of addiction. Although no one directly mentions the moral model, the way they viewed people who use drugs and explained relapse demonstrates the continued existence of the moral model of addiction in Vietnam. The general perceptions of drug use and addiction result in negative, judgemental attitudes towards people who use drugs among research participants, which contradict a non-judgemental approach of authentic harm reduction (people morally judge those taking drugs before helping them). 
People who use drugs were commonly seen as potential harms to society who are in need of harsh punishment and control.

In general, study participants demonstrated that abstinence-based mind-sets remain popular. As a result, this has led to an emphasis on individualised medical solutions to prevent drug use and relapse, generally ignoring the complexities of drug use behaviours and the intersections between drug use with social and environmental factors. Focusing on individualised solutions supports the idea of the 06 centres if people have the 'disease' of 'addiction'. This approach is seen as a method to reduce harm caused by drug use and people who use drugs from Vietnamese perspectives. The current perceived harms have led to distinct differences between pseudo harm reduction interventions and an authentic harm reduction philosophy. In Vietnam, harm reduction is seen a method to control drug use and people who use drugs in order to protect the wider community, while philosophically harm reduction does not necessarily mean reducing drug use; rather it entails promoting people who use drugs' rights (as discussed above). The next chapter illustrates what research respondents think should be done to respond to drug use. These findings further demonstrate that stakeholders frequently perceive that harms can be limited through reductions in the demand and supply of drugs. Accordingly, enforced abstinence, not harm reduction approaches, are dominant in Vietnam. 


\section{Chapter Eight: Findings on Responses to Drug Use}

\section{Introduction}

The previous findings showed that many professionals in Vietnam had problematic understandings about drug use, addiction, and people who use drugs. Working from misinformation, they tended to promote punitive control measures to reduce the harms from drug use. This chapter further demonstrates that abstinence-based approaches have a strong influence, as many believed that reducing harm means developing measures to reduce the supply and demand of drugs. Regarding supply, an overwhelming number of respondents thought that reducing harm means controlling and eradicating the supply of drugs and increasing punishments for those possessing, trading or smuggling illicit drugs or precursors. Meanwhile, for demand reduction, respondents proposed methods to control and punish people who use drugs and to treat addiction through detoxification, isolation, re-education, and medical interventions. For police and MOLISA professionals, compulsory treatment 06 centres were deemed an effective method for both controlling people who use drugs and treating addiction in Vietnam.

The existence of 06 centres remains, however, controversial. While police and MOLISA officials advocated for them, others (especially those working in international or public health sectors) criticised the centres for being ineffective, costly, and having poor services and high relapse rates. Some interviewees revealed that drugs still exist in the centres; staff are unqualified; levels of blood-borne diseases are high; and vocational training programmes are forms of forced labour that do not prepare people for the employment market. They argued that the centres are retained for political and financial reasons. This chapter reconfirms that abstinence-based views are widely held among study participants. However, it also illustrates some progressive thinking related to treatment. Many professionals, including police and MOLISA workers, proposed various methods for treatment, such as not isolating people who use drugs, allowing people who use drugs the right to decide their treatment, and providing social and psychological support. These results demonstrate that the promotion of harm reduction could still be possible in Vietnam. 


\section{Supply Reduction}

Given the widespread perceptions of harm caused by illicit drugs, over half of the interviewees from different agencies emphasised the importance of controlling illicit drugs in order to reduce harm. They argued that drug problems can be addressed if stricter punishments are imposed for those who possess, trade, traffic or smuggle drugs, or produce precursors. In other words, they asserted that if the drug supply is controlled, the harms associated with drug use can be reduced:

We need to increase punishments for drug trading, trafficking, smuggling and producing precursors because I don't want Vietnam to be a logistics area for drug production. We also need to more strictly punish people who possess, trade, produce and smuggle synthetic drugs (Police 1).

In general, if we want to detect drug use behaviour we need to have some forms of handling measures in order to control drug use and implement some interventions (Social worker trainer).

Survey findings also supported the importance of controlling drugs, especially drug supply. A large number of survey respondents favoured a solution that 'We can protect society by eradicating the supply of drugs', with $87 \%(n=219)$ either agreeing or strongly agreeing with this solution (see Figure 8.1). 
Figure 8.1: We can protect society by eradicating the supply of drugs

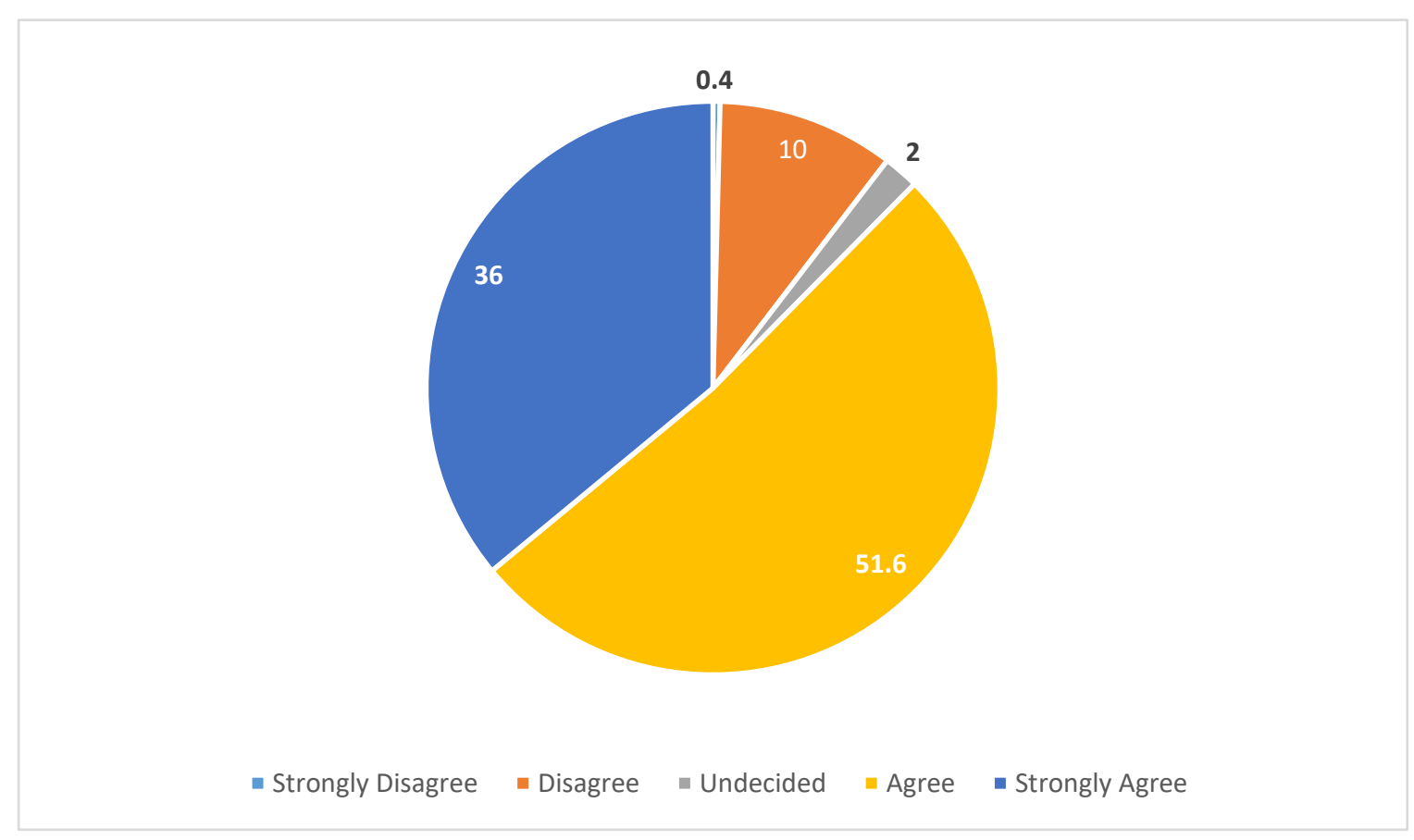

These findings could be anticipated, given the dominance of abstinence-based mind-sets and approaches among Vietnamese people, discussed previously.

These findings demonstrate the prevalence of abstinence-based approaches. In addition, over $90 \%$ ( $n=227)$ of survey respondents agreed or strongly agreed that we should create a drugfree society (see Figure 8.3).

Figure 8.2: We should create a society free from drugs

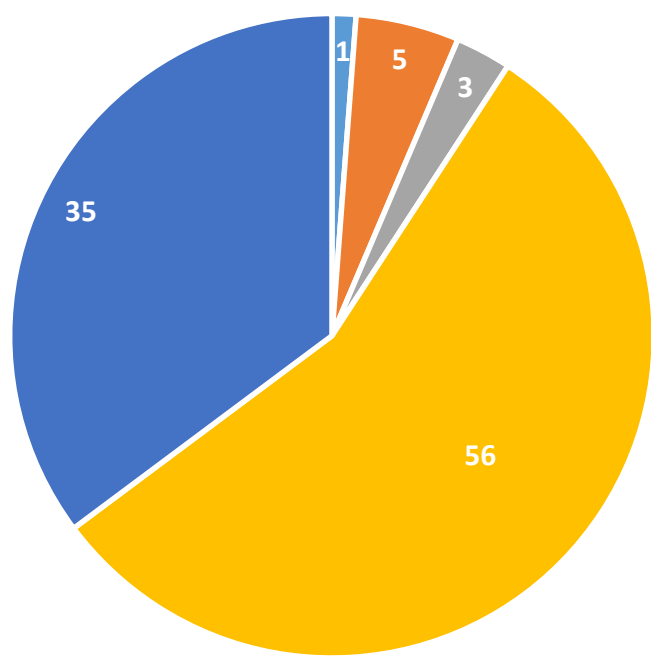

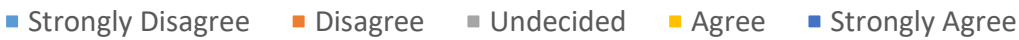


In summary, the overwhelming majority of interview and survey respondents believed that reducing harm means controlling and eradicating the supply, and increasing punishments for those possessing, trading or smuggling illicit drugs, or producing precursors. This reflects government policy. By imposing stricter regulations and law enforcement, workers believed that Vietnam will address the root causes of drug use problems, and reduce harms (Windle, 2012). This thinking is common, but supply reduction cannot be seen as harm reduction. As discussed in Chapter Two, many policy-makers view that the best way to address drug use problems is to reduce the prevalence of illicit drugs (supply reduction) (Stevens et al., 2010). For them, illicit drugs cannot cause harm to society if they are not actually in that society. This justifies prohibition. However, prohibition generally fails to reduce supply, and it produces and exacerbates drug-related harms as discussed in Chapters Two and Three. Indeed, prohibition remains the greatest contributor to harm, such as drug user stigma, social dislocation or drug market violence (Csete et al., 2016; Global Commission on Drug Policy, 2014, 2017; Levy, 2014; Werb et al., 2011). Despite this, preventing drug use is still an overarching goal for many professionals.

\section{Demand Reduction}

Most research participants believed that promoting demand reduction interventions will reduce supply and the harms associated with drug use, particularly by controlling people who use drugs and addressing addiction. The existence of 06 centres was regularly seen as an answer for the latter. Yet there were different opinions among research participants about the effectiveness of the centres.

\section{Controlling People who use drugs}

Over half of interview participants pointed out that stricter controls of people who use drugs are needed to protect society because, for them, people who use drugs are potentially harmful to society and the wider community. As Stevens et al. (2010) explain, from this perspective, the best way to reduce harm caused by people who use drugs is to reduce the number of people who use drugs in society. Many interviewees were concerned about how the government could manage and control people who use drugs. Some explained: 
We need to educate and control addicts and keep them away from drugs and so they do not relapse ... without control, 'scummy junkies' can have criminal behaviour (Doctor 5).

Drug user is a special category that needs strict controls ... We also need to make it easier to send people who use drugs to treatment centres, which I believe will reduce the demand side. And by reducing the number of people who use drugs in society, crime related drugs and drug use will decrease (Local police 4).

We need to continue implementing the administrative law to control addicts ... that they need to give up drugs. If not, they will be subjected to administrative sanctions, even being sent to prison as in other countries (MOLISA official 1).

The majority of survey participants also favoured criminalising drug possession. Over $75 \%$ ( $n=195$ ) believed that 'we can protect society by punishing drug possession' (see Figure 8.4).

Figure 8.3: We can protect society by punishing drug possession

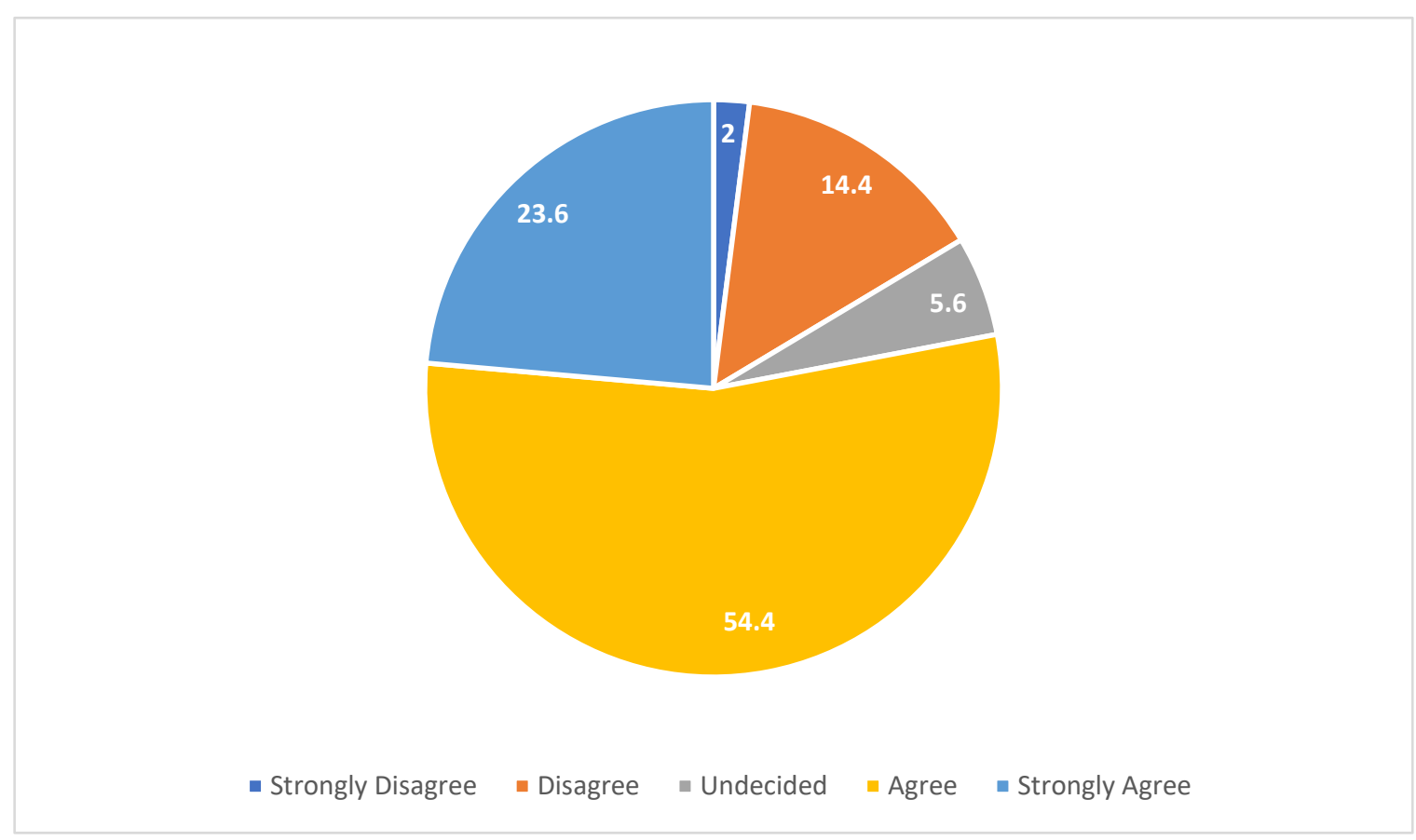

Similarly, over $75 \%(n=19)$ of interviewees did not advocate for the decriminalisation of drug possession for personal use. They argued that people who use drugs may take advantage of decriminalisation to trade illicit drugs. Further, they thought that punishing people who possess drugs is a necessary measure to prevent drug use: 
Drug possession is still crime ... there is a very narrow gap between possessing drugs for using or other purposes. Or, to put it another way, the needs for personal use and needs for selling drugs or committing crimes to get money are so close ... possessing drugs needs to be seen as a crime so that we can have better prevention (Police 4).

They [people who use drugs] can possess an allowed amount of drugs and then sell it ... they can sell drugs even just with small doses (Doctor 1 ).

I think if we detect someone using drugs, they have to receive some sanctions, or they have to go to a drug court. So, people who use drugs need to be administratively sanctioned, such as by a fine, or having to join drug treatment (Social worker trainer).

In addition to criminalising people who use drugs, many research participants thought that an effective method to control people who use drugs is to force them to join drug treatment. As discussed previously, Windle (2016) clarifies that addiction treatment is commonly perceived to reduce the threats that people who use drugs can pose to the wider community. The survey data confirmed this notion. Over $70 \%(n=178)$ of respondents agreed or strongly agreed that 'people who use drugs need to be sent to treatment centres' (see Figure 8.5). 
Figure 8.4: People who use drugs need to be forced to get treatment

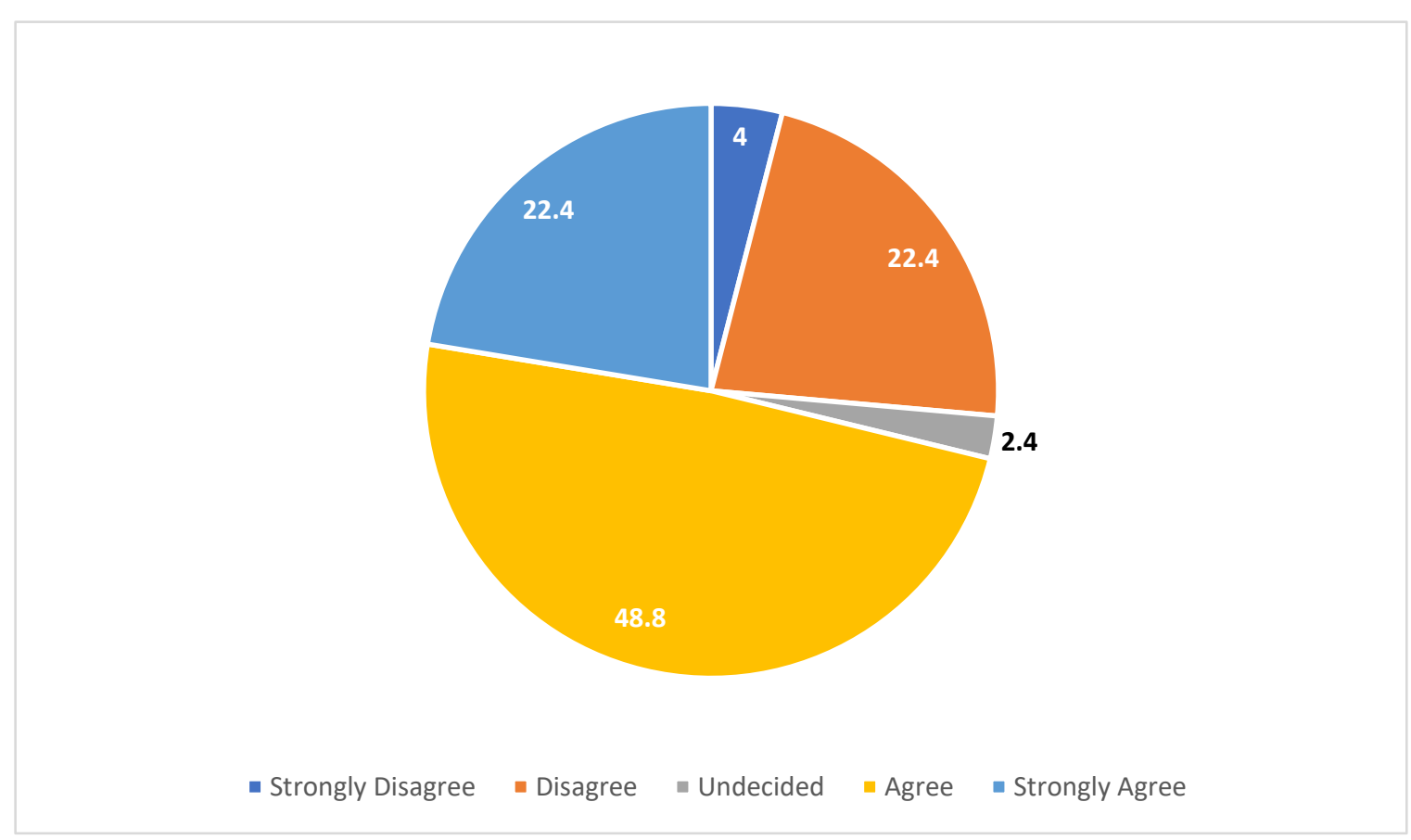

In general, professionals tended to think that forcibly treating people who use drugs or even punishing them is necessary to reduce harm to society. Nevertheless, as already discussed, the tight control over people who use drugs and overly punitive laws have caused stigma and discrimination rather than providing people who use drugs with assistance (Global Commission on Drug Policy, 2014). Also, discriminating against and controlling people who use drugs are contrary to an authentic harm reduction philosophy, in which people who use drugs' opinions are respected and controlling or forced treatment are unacceptable (Marlatt \& Witkiewitz, 2010).

\section{Treating Addiction}

As analysed in the previous chapter, a mixed version of the moral and brain disease model of addiction was popular among interviewees, and this section will further demonstrate this mix. Every interviewee had thoughts on how to treat addiction. Some emphasised re-education, and detoxification or abstinence, while others deemed that medication-assisted therapies are needed. Only some recommended a comprehensive approach, ranging from medicationassisted therapies to psychological or social therapies. Interesting, none favoured a one-sizefits-all approach because, they argued, different people who use drugs need different methods to overcome their addiction. For instance: 
There is no common method for all addicts ... this model may be good with some addicts, but it is not necessarily appropriate with others, it depends on their personalities and their emotional feelings (Doctor 5).

We don't have the best method for treating addiction, there are many ways actually. Some can engage in voluntary treatment, some can join community-based treatment, some can get over addiction by themselves, or they can use methadone. Treatment varies from person to person, there is no one-size-fits-all solution here (International worker 6).

Although a uniform approach is not supported in Vietnam, addiction treatments are not individually tailored. In fact, people who use drugs go through the same processes when they are engaged into treatment, including entry procedures, detoxification, re-education, therapeutic labour, long-term follow-up and community reintegration (see Chapter Five). Research participants' perspectives were not illustrated in current practice.

Study interviewees also highlighted different methods for treating addiction. As outlined below, some emphasised detoxification in order to help people who use drugs remove harmful substances from their body and others thought isolating people who use drugs was necessary to keep them away from drug use environments. Meanwhile, some proposed that people who use drugs need to be re-educated to be 'better' persons while others highlighted the importance of medical interventions.

\section{Detoxification}

'Detoxification' was highlighted by many interviewees as an important first measure. They believed that people who use drugs need to go through a detoxification process to eliminate harmful substances from their bodies. For them, this measure can reduce harm caused by drugs and help people who use drugs to recover. For example:

The best way to treat addiction is that we need [a] detoxification process based on the standards of [the] Ministry of Health in order to alter addicts' eating and living habits (Local authority). 
Detoxification helps drug addicts to remove harmful effects of drugs, and treat addiction ... helping them to recover their health and giving up drugs later on (Local police 1).

Many interviewees thought detoxification will help people who use drugs to overcome withdrawal symptoms and recover their health. This method is preferred because, according to MOLISA (2015), it helps people who use drugs to be 'clean' or 'free' from drugs which is important to stop relapse. Nevertheless, although all those in treatment centres have to undergo detoxification processes, the relapse rate (an estimated 90\%) is extremely high (Human Rights Watch, 2011; Kamarulzaman \& McBrayer, 2015). Despite this lack of effectiveness, detoxification remains the foremost method to treat addiction in Vietnam. It is combined with an isolation-based approach in which users are detained in treatment centres.

\section{Isolation}

In line with 'detoxification', some interviewees emphasised the importance of isolating people who use drugs from society for a certain period of time. Some even suggested that an isolation period of two years is necessary to facilitate treatment processes. For them, without isolation, successful treatment can be difficult:

If we are not isolating addicts from the drug using environment, they will use drugs again. Thereby, complete isolation is a must (Police 6).

We should isolate addicts from the drug using environment, because if we allow them to be exposed to the environment, they are going to relapse (MOLISA official 1).

Yet their responses generally did not consider what will happen when people who use drugs return to society. As noted previously, the majority of people who use drugs have encountered a high level of stigma and discrimination because they are judged to have poor moral character. As a result, many of them end up using drugs again as a response to forget or overcome social dislocation. Isolation is probably a temporary solution for some users, but it is not for every user. 
The survey data also strongly supports this abstinence-based approach with the majority (over $70 \%, n=177$ )) of survey respondents agreeing or strongly agreeing with the statement 'people who use drugs need to quit all drugs and stay drug-free' (see Figure 8.7).

Figure 8.5: People who use drugs need to quit all drugs and stay drug-free

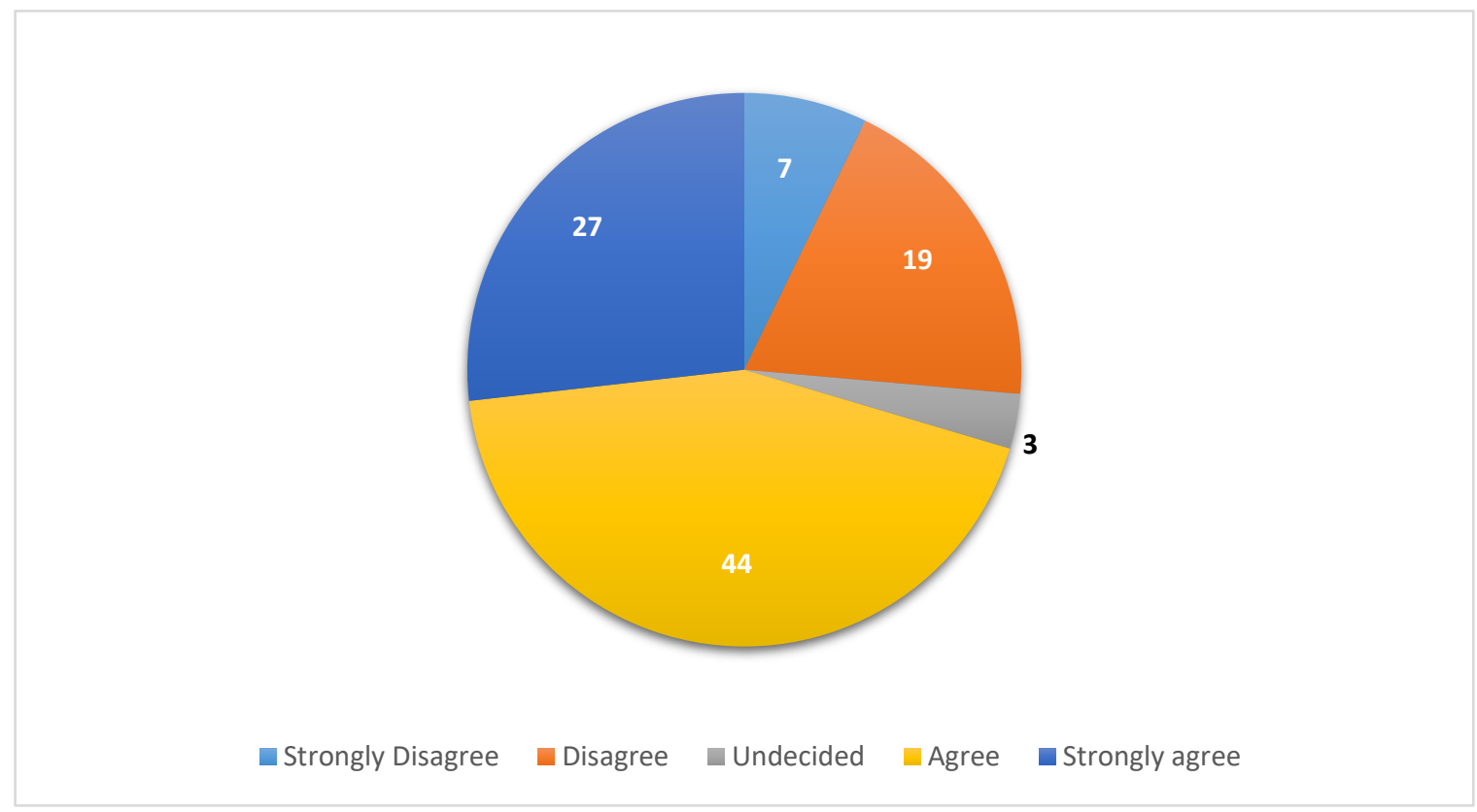

Nevertheless, some interviewees disagreed with the isolation of people who use drugs. They argued that, instead of becoming a better person, people who use drugs would be 'better criminals', if isolated. For example, some interviewees explained:

I don't necessarily think that isolating addicts will be effective, they will be influenced and learn others' bad habits. When they first come in the centres they just know some ways to use drugs, but later on they know how to consume different types of drugs or where they can get drugs for feeding their habit (Consultant).

They [addicts] don't always necessarily come out in a better way, they may come out actually learning to be a better criminal. And I don't mean being a better drug user, I mean being an actual criminal (International official 1 ).

Their opinions reinforce the drugs and crime link, which is supported by the majority of Vietnamese leaders and researchers. Yet as discussed previously, the link between drugs and crime is ambiguous and socially and politically constructed, rather than evidence. Vietnam 
still believes that isolating people who use drugs is an effective way to prevent crime and relapse.

Nevertheless, as shown previously, isolating people who use drugs from their community only makes it more difficult for them to stabilise their lives (Windle, 2015). People who use drugs are isolated and forced into treatment simply because the government believes this will protect society and ensure social order (Hong et al., 2004). Yet from a harm reduction perspective, isolating people who use drugs threatens their freedom and violates their autonomy (Elliott et al., 2005). Although isolating people who use drugs has many counterproductive effects, the Vietnamese government still implements it on the grounds of reducing crime, treating addiction, and re-educating people who use drugs.

\section{Re-education}

About half of the interviewees emphasised a re-education-based approach as an important step to help people who use drugs to become 'a good person'. For example:

Re-education is to help addicts to alter their consciousness and modify their behaviour, which will reduce harm caused by addiction, and reduce illegal drug use (MOLISA official 4).

Addiction is not only treated by medicines, but it needs a comprehensive approach, including social-psychological interventions, morality, and medication (Government official 1).

In terms of treating drug addiction, medicine and medication only play supportive roles, re-education should be the main focus (Doctor 5 ).

Their options affirm that people who use drugs are problematic, not the society or the system. This is linked with a middle-class perspective of morality and correct behaviour, which was discussed in Chapter Three. The focus on moral perspectives generally results in greater stigma of and discrimination against people who use drugs.

Furthermore, the emphasis on morally re-educating people who use drugs reflects the moral model of addiction, which condemns people who use drugs as 'bad people' who have low 
moral standards and violate social conventions (Hong et al., 2011). This approach causes stigma towards people who use drugs and makes it harder for them to find employment or a place in society (Tomori et al., 2014). In fact, there has been no evidence showing that reeducation can make Vietnamese people who use drugs become 'better' people. These results reflect a moral model of addiction and abstinence, and they generally go against an authentic harm reduction philosophy based on a non-judgemental approach to people who use drugs.

\section{Medical Interventions}

Although the majority of the interviewees considered detoxification, isolation and rehabilitation as the essential components of addiction treatment, many of them (two thirds, $\mathrm{n}=17$ ) also proposed medication-assisted interventions to treat addiction. Most agreed that substitution treatment is an effective method. They mainly referred to methadone or buprenorphine for treating addiction:

Methadone is an effective method in resolving drug addiction, it is a substitute substance that is less harmful than other drugs (heroin). This drug can treat addiction to people who use drugs for a long time, even lifelong (Local police 2).

As for addictive substances that had substitute ones, we should best prioritise substitution treatment. For example, regarding heroin addicts, we can use Methadone and buprenorphine for substitution (International worker 6).

Their perceptions generally reflected the brain disease model of addiction. Their ideas also mirrored the norms of Vietnam's pseudo harm reduction interventions that have been discussed previously. Yet only considering some medical interventions to treat addiction continues to confirm that harm reduction is viewed from a public health perspective among Vietnamese key stakeholders. The importance of other legal, social, psychological, and environmental factors was generally not mentioned.

In summary, there was a wide range of suggestions from interview participants for treating addiction. Some emphasised re-education, and detoxification or abstinence, while others deemed that medication-assisted therapies are needed. The majority, however, emphasised individual-based solutions. As Nguyen and Scannapieco (2008) explain, from Vietnamese 
perspectives, addiction is largely determined as resulting from the failings of the individual, and their biological or psychological defects. Accordingly, the solutions for addiction are generally placed at the personal level, rather than considering social and environmental issues. These results further confirm that the moral and disease models of addiction are widespread, and that abstinence-based approaches are still preferred for treating addiction, despite their ineffectiveness. Accordingly, for many interviewees, especially police and MOLISA professionals, compulsory treatment centres were still seen as an effective method for both controlling people who use drugs and treating addiction.

\section{Compulsory Treatment Centres}

One of the most popular methods to treat addiction in Vietnam is to send people who use drugs to compulsory treatment centres. Some interviewees, especially MOLISA officials, described compulsory treatment centres as multifunctional centres, for instance:

In Vietnam, we no longer have a concept called '06 centres', instead, $100 \%$ of the centres have changed their functions to be multifunctional addiction treatment units, which include voluntary treatment, compulsory treatment, and Methadone treatment (MOLISA official 4).

Some international interviewees revealed that the main functions of the centres are to provide people who use drugs with detoxification therapy over 7-10 days and then to morally re-educate users rather than engage in any treatment per se. One interviewee noted:

My understanding is that it's a rehabilitation and education centre. It is not a drug treatment centre ... I often get told that the direct translation is rehabilitation and education centre. So it's basically a re-education camp (International worker 1).

One MOLISA professional accepted the fact that the main functions of 06 centres are to control and detain people who use drugs, instead of providing support services:

06 centres are mainly to control and re-educate addicts rather than provide them with other medical treatment and social therapies (MOLISA official 2). 
Apparently, compulsory treatment and rehabilitation are the main components of the centres, rather than social and psychological supports. Further, the 06 system was criticised by many interviewees for its ineffectiveness and causing many problems to people who use drugs.

\section{Problems of the Centres}

The existence of the 06 centres was criticised by many research participants. The majority of critics elaborated on their multiple problems, and they called for their closure or transformation. They determined that the centres are costly and ineffective:

It [the 06 centre] was very expensive ... It wasn't really solving the problem or addressing the problem (International worker 5).

We don't endorse the existence of compulsory and concentrated detoxification because it costs a lot of state money, but it does not work ... When we open such a centre we need a lot of human resources who provide services for people who use drugs ... we need to invest a huge amount of money, but we do not get good outcomes (Harm reduction official 2)

The main function of the centres is to treat addiction and to prevent relapse, but some interviewees argued that the centres just keep people who use drugs away from the community rather than providing them with treatment. As a result, centres are characterised as ineffective with relapse rates being very high:

They just detain addicts ... They think that if they lock addicts up for two years, addicts will quit drugs then. But what I can see is that many people come back to the centres once, two times, but many times actually (International worker 5).

People think that sending addicts to 06 centres to detoxify and isolate them from society will help them give up drugs. In fact, these approaches are not really effective when almost all drug addicts use drugs again after they are released from the centre (Social work trainer). 
The majority of people who use drugs when coming out they relapse ... they don't get necessary support and consultation in a way that provides them with the motivation to make changes. So, when they return they continue to relapse (Consultant).

The survey data reveals different ideas as to whether the compulsory treatment centres (06 centres) are effective in preventing relapse or not. Nearly $47 \%(n=117)$ survey respondents agreed or strongly agreed with their effectiveness, while over $41 \%$ ( $n=103$ ) disagreed (see Figure 8.8). This demonstrates a significant ambivalence about the usefulness of 06 centres.

Figure 8.6: Compulsory treatment is effective in preventing drug relapse

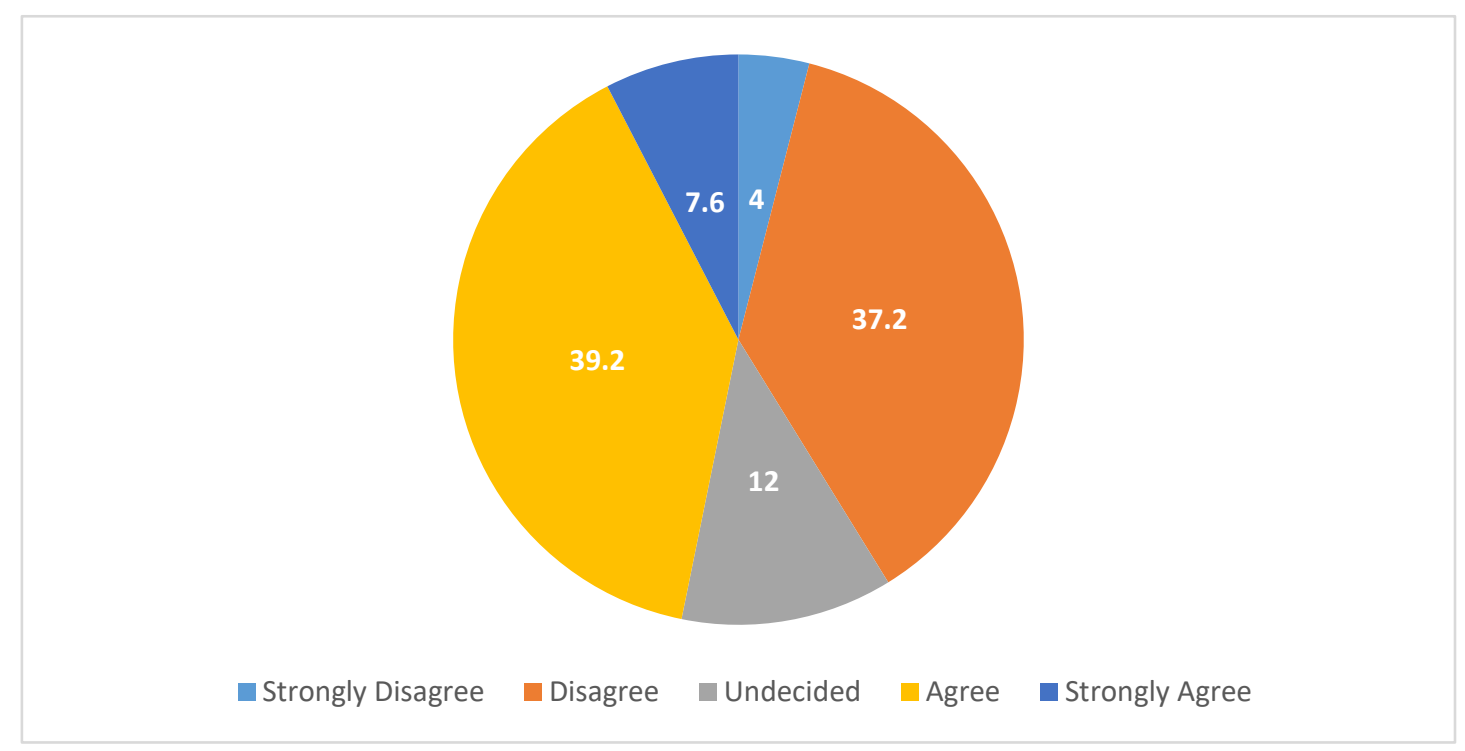

Another criticism of 06 centres was that clinical care is poor, and the minimal treatment services are not based on best practices or scientific evidence. Some interviewees claimed that the risk of contracting infectious diseases in the centres is high:

I've been to many. There aren't treatment services. There aren't clinical staff. There might be a doctor, and they might give them some medicine for detoxification, but detox, first of all, [it] isn't drug treatment. It's not necessarily needed for every patient (International official 1).

The majority of the centres are not clean, they pose many risks ... for example, addicts have no diseases like HIV or Hep C before entering the centres, but then when they come out they have got some (Harm reduction official 3). 
While some interviewees stated that the centres are good because they provide vocational and skills training, others believed that 'vocational training programmes' are themselves compulsory labour. For them, the types of vocational training on offer are not useful in preparing individuals for the employment market. Notably, some police and MOLISA officials also accepted this fact:

A very small percentage of the people that go there actually learn a skill, but I'm not so sure that the skill they learn is an employable skill outside. Some places, as you know, probably not, they shuck cashew nuts, they make eyelashes, they put electrical plugs together, they sew uniforms together, but if none of those things exists in the community where they're from, that's not a skill that they can use (International worker 2).

The centres offer some vocational training ... but their training programmes are not applicable to the labour market ... as a result, for many addicts, when they return to society, they often fail to find a job (Police 6).

We offer much vocational training, but it seems to be hard to meet all addicts' needs. For example, people who are from the countryside are different those who are from the city with some training, such as making false eyelashes or tailoring are hard to find a decent job outside (MOLISA official 1).

Further, although the aim of the centres is to keep people who use drugs away from drugs, many participants revealed that drugs still exist in the centres. This issue generally goes against government commitments to create a 'clean' environment in the centres. Some interviewees even stated that drug smuggling is backed by some centre staff:

The majority of 06 centres are not 'clean' as we expected, as many addicts still have drugs to use. This drug smuggling is not only conducted by addicts, but rather by many staff (Harm reduction official 1).

I am totally in opposition of rehab centres because they are not a good method for treating addiction, in fact, in the centres, heroin and other drugs are smuggled by guardians (International worker 2). 
In many centres, staff even sell drugs because we fail to keep the centres completely clean ... there are still outside relationships, so drugs are still trafficked in the centres, addicts still have drugs to take (Police 4).

Many interviewees also raised concerns over the staff at the centres, such as a lack of professional knowledge and lack of patience and sympathy towards people who use drugs. Some articulated:

They [staff] don't know much about drug treatment, so their performance is poor ... wage and salary policies are not really adequate, just a few hundred thousand Vietnam Dong per month [around 30 USD] (Doctor 6).

In the centres, violence is common, staff put a lot of pressure on patients, so the patients don't want to continue treatment and stay there. They [staff and patients] fail to find a common voice (Consultant).

The roles of consultants and service providers are minor ... 50\% of them have never been trained to treat addiction, their tasks are only to administer and detain addicts ... So the quality of the treatment is poor (Police 1 ).

The discrimination from service providers runs counter to the promotion of authentic harm reduction. This is because as noted previously, non-judgemental attitudes are the core component of harm reduction strategies, so a lack of patience and sympathy towards people who use drugs will generally make them distrustful, and drive them away from harm reduction services.

In summary, interviewees had plenty of criticisms about the 06 system. The centres were blamed for being ineffective and costly with high relapse rates. Although the main function of the centres is to treat addiction, clinical care is seen as poor, with minimal treatment services and high infectious disease rates. Further, with drugs still in circulation, the centres are not 'clean', as promised by the government. Some vocational training is provided, but it is argued to be 'forced labour' that does not help future employment prospects (Human Rights Watch, 2012). Unqualified staff focus on detaining people who use drugs, rather than providing them with assistance. These results are similar to those from previous studies, 
discussed in Chapter Five (Amon, Pearshouse, Cohen, \& Schleifer, 2014; Human Rights Watch, 2011, 2012; Kamarulzaman \& McBrayer, 2015; UNAIDS, 2012). These studies also show that the existence of the 06 system remains the biggest barrier for expanding harm reduction in Vietnam, but many government workers (mainly police and MOLISA staffers) still supported the centres.

\section{Justifications for the Existence of the Centres}

The results showed that the main function of 06 centres is to detain people who use drugs rather than provide them with treatment and necessary assistance. Yet there were many justifications for keeping the centres, especially from police and MOLISA officials. Their main arguments were that the centres are necessary for keeping people who use drugs away from the temptations of the outside world; protecting the wider community; and assisting users or helping their family. For instance:

The centres are helping drug addicts to stop cravings and detoxify drugs, treat disease, educate and provide educational training ... sending drug addicts to the centres is a humanitarian policy because our government provides funding for operating the centres and the centres are helping addicts to recover health and give up drugs later on. The strength of 06 centres is to isolate users from drugs and help them to recover and ensure safety and social order for society. Many families are willing to send their loved ones to 06 centres because they cannot afford private rehabilitation centres and they cannot control their family members at home (Police 1).

The main strength of the centre is that it helps to ensure social safety, the addict's family can save a huge amount of money for treatment (Doctor 5 ).

The centres are providing people who use drugs with a much better service in terms of detoxification without causing any problems and side-effects, compared to local clinics, which lack facilities and qualified staff (MOLISA official 5).

Other participants from public health and international sectors explained that many people are keen on the 06 system as they think it will increase social order and safety: 
The main benefit of the centre is to detain addicts so that they no longer break laws or affect the community (Consultant 1).

First of all, it isolates the person from the community. The community doesn't have anybody stealing anything in the community. For that time period, it seems great. The family thinks, because the government and the system has said that these are treatment programs, thinks they're actually sending their family member to a good place because they're getting help. On top of that, the family member wants a break. They don't want to have to live in this situation either. And sending him away for two years, three years, or whatever it is, gives them that break. And they think they're doing good for their patients, or their family members (International worker 1).

Notably, some interviewees pointed out economic and employment reasons on why the 06 system still exists regardless of their failings to treat addiction and prevent relapse:

There are currently plenty of personnel and facilities in the centre ... there are hundreds of people ... each centre has hundreds of employees, and we have more than 120 centres ... if we transform, where they will work then? (International worker 7).

Vietnam still maintains the 06 centres because if they close the centres, many staff, guardians, doctors, nurses have nowhere to go (International worker 3 ).

From their opinions, it can be seen that the wellbeing of centre staff, and economic incentives for centre leaders, are likely political reasons for maintaining 06 centres in Vietnam.

In general, these results are consistent with previous arguments about the existence of 06 centres. Some respondents identified that the centres are ineffective in their treatment approach, and have counterproductive effects by limiting people who use drugs' access to health care and harm reduction services. This study also adds a new dimension in its identification of the political reluctance to close the centres on employment or industry grounds. At the same time, many participants, especially police and MOLISA professionals, supported the centres as they believed that centres help users to overcome addiction, and provide them with vocational training. They argued that the centres contribute to reducing 
crime rates, ensuring social order and safety, while protecting the wider community from people who use drugs. What is clear is that the 06 system is largely justified on abstinence grounds, and is promoted by political factors rather than 'scientific evidence' ${ }^{75}$

\section{Wider Perspectives on Treatment}

Although the majority of study participants followed abstinence-based approaches, some informants, including police and MOLISA professionals, also proposed alternative methods for treatment. Their suggestions included avoiding isolation, considering social and psychological factors (especially the involvement of family and society), allowing people who use drugs the right to decide their treatment, and providing comprehensive support.

Over two thirds ( $n=19 / 26)$ of interviewees did not favour an isolation-based approach, because they claimed that this approach is not effective and cannot address the problem in the long-term. In other words, they believed isolation is not an answer to treat addiction, particularly as over $90 \%$ of users take drugs again after they come out:

Addiction is a brain disease, so no matter how long you isolate addicts they will still relapse as they come out ... they use drugs again immediately ... isolation is not effective in terms of medical practice and economics because we need a lot of money to maintain such an approach (Doctor 6).

I don't necessarily think, clearly, that rounding up people who use drugs ... and putting them into a camp ... it's not going to help society. It may get them away from the community, and it may get them away from their family, but it's not going to resolve the problem because the minute they come out $95 \%$ are going to use again (International worker 1).

\footnotetext{
75 The 06 centres are used to control crime rates in Vietnam (Vuong et al., 2016). However, as noted in Chapter Two, many people who use drugs have been offenders anyway, so determining those offenders whose offences relate to drugs and those that do not is almost impossible (Bean, 2014). Also, the 06 centre-based approach seems to deny the role of inequality in generating crimes, and importantly it tends to place the blame squarely on people who use drugs for any offences they commit (Stevens, 2007).
} 
Their perspectives contradict those who support 06 centres. As noted earlier, the role of the centres is to keep people who use drugs away from drugs and help them to stop using drugs, but, obviously, the vast majority of centre detainees take drugs immediately after being released. This questions the effectiveness of the centres and the commitment the Vietnamese government provide to people who use drugs' families when enforcing people who use drugs to join the centres. As noted previously, forcing will never work when it comes to drug addiction treatment. Instead, people who use drugs need help and support from society to overcome their problems.

Some interviewees emphasised the vital nature of society support in assisting people who use drugs. As noted in Chapter Three, civil society plays an essential role in shaping and influencing drug use and addiction. As some interviewees explained:

We should find ways that promote the role of youth and women associations, schools, and other organisations in order to find out what happened and is happening to people who use drugs before providing help ... we should create job opportunities and better social-economic conditions for people who use drugs (Police 3).

I think in the treatment process, we should not isolate people who use drugs from society (the community, family and working places) because society plays an important role in fostering and supporting people who use drugs (Social work trainer).

In addition, some interviewees highlighted the need for engaging family in the treatment process. For example:

Medicines are not enough, importantly we should have psychological interventions and family support, especially the trust of family is a very important element (International worker 3).

We provide patients' family with consultation, and information about their family members ... if family trust that patients are able to make changes, they will feel happy and continue to improve and follow treatment (Consultant). 
Notably, some participants noted the importance of giving people who use drugs rights to decide what they need for their treatment rather than forcing them to follow service providers' requirements:

We can help people who use drugs by allowing them to decide and build treatment plans ... it will be hard for them to follow our treatment programmes ... we should be just supporters (MOLISA official 5).

We should consider people who use drugs as the key component in the treatment process. Vietnam needs to place people who use drugs at the centre of the treatment (International official 2).

These views on respecting the rights of people who use drugs in treatment are remarkable within a Vietnamese context in which people who use drugs are seen as a high-risk group. The voice of people who use drugs is generally underestimated because, as noted previously, they are blamed for having low moral standards and contributing nothing to society. Therefore, giving people who use drugs the right to decide their treatment is an important step for developing authentic harm reduction in Vietnam.

The survey also shows this positive perspective on the user's role in addiction treatment. A large proportion of survey participants $(68 \%, n=170)$ agreed or strongly agreed that treatment plans and goals should be set by people who use drugs (see Figure 8.10). 
Figure 8.7: People who use drugs should play a central role in deciding their treatment goals

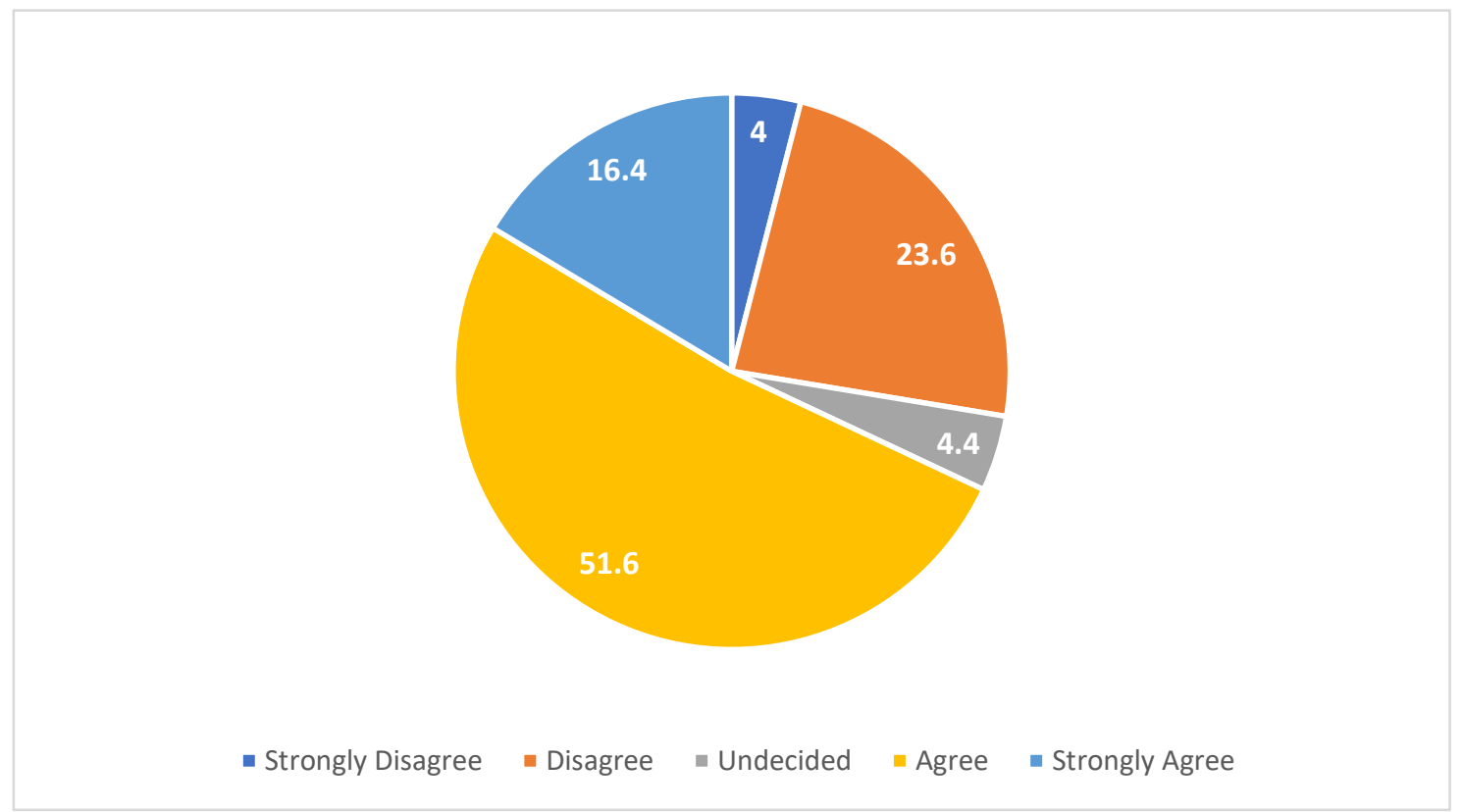

Interestingly, although the majority of participants asserted that addiction is a chronic disease, many of them emphasised the value of a comprehensive approach, involving medication-based therapies, and psychological and social therapies. For them, treatment needs to address all problems related to people who use drugs, including biological, psychological and social functions. For example, some interviewees stated:

Relapse is normal ... using medication is not enough, we need to use psychological and social measures (Government official 1).

We need to develop a comprehensive method for treatment. We should provide people who use drugs with social supports, such as training, offering jobs, peer education or psychological support (Social worker trainer).

These ideas are close to authentic harm reduction principles, which consider different types of support for people who use drugs in order to reduce harm caused by drug use.

The quantitative data also confirm these arguments, highlighting a wide-ranging approach to drug treatment. Nearly $50 \%(n=123)$ of survey respondents disagreed or strongly disagreed that 'abstaining from drugs is the only effective treatment for people who use drugs' (see Figure 8.11). 
Figure 8.8: Abstaining from drugs is the only effective treatment for people who use drugs

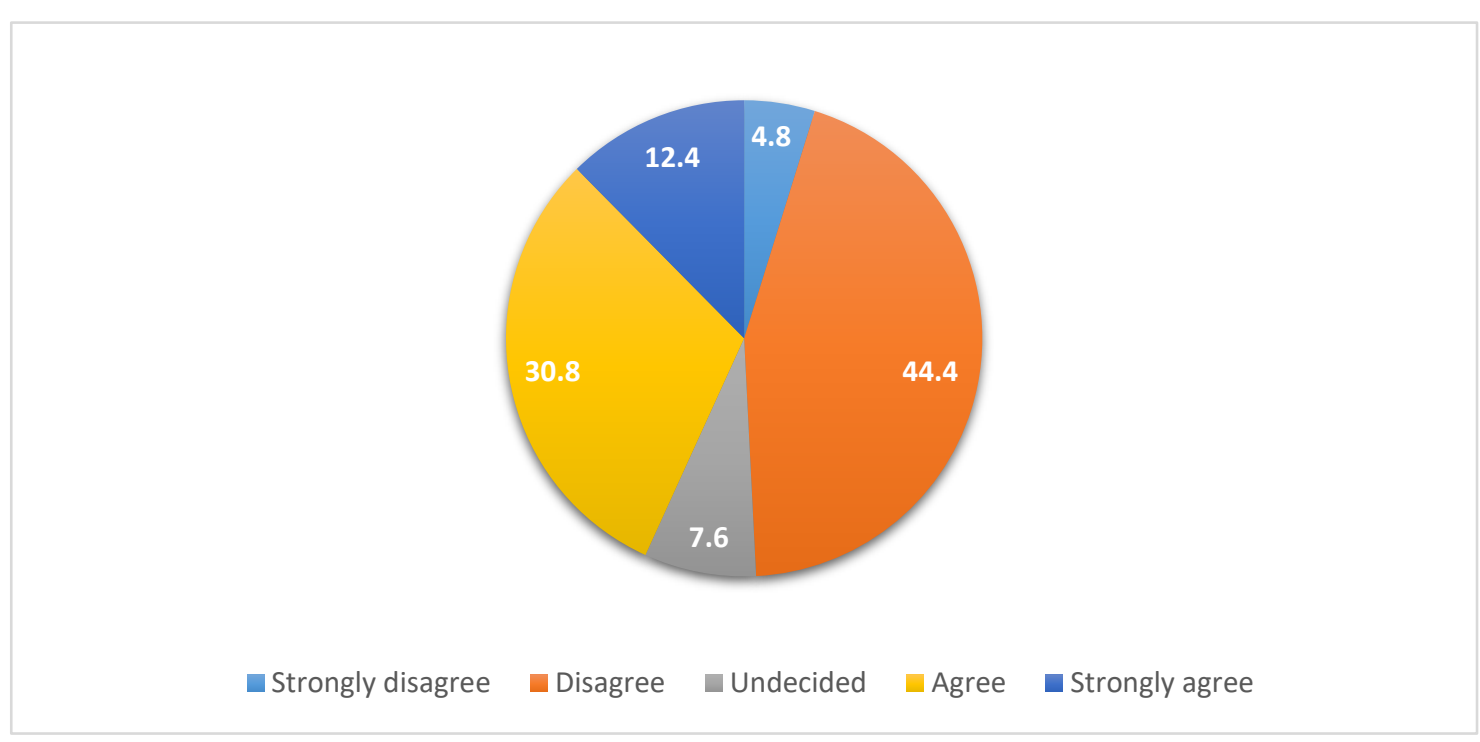

These results are interesting, given the fact that most recommended abstinence. As noted in Chapter Seven, almost all interviewees highlighted social and psychological factors to explain why people take drugs, so these factors likely affected their perception of why abstaining from drugs is not the only effective treatment for people who use drugs.

In short, some interviewees considered psychological and social factors as being significant to treatment processes. As argued above, addiction is a psychological and social problem rather than a medical one, so addressing these concerns is vital. While, in practice, Vietnam's treatment processes follow a one-size-fits-all approach, most study participants agreed that people who use drugs should be allowed to decide their treatment. Many interviewees highlighted the importance of providing people who use drugs with various supports, including medication, psychological and social therapies. These perspectives are significant to facilitating treatment based on authentic harm reduction principles in Vietnam.

\section{Conclusion}

The majority of interview and survey respondents believed that reducing harm means eradicating the supply and increasing punishments for those possessing, trading, and smuggling illicit drugs or producing precursors. Participants tended to think that controlling and punishing people who use drugs is necessary to reduce harm to society, and the best way, according to many informants, is to send people who use drugs to forced treatment. 
Regarding treatment, some emphasised re-education, and 'detoxification' or 'abstinencebased approaches', while others deemed that medication-assisted therapies are needed. Also, due to the prevalence of abstinence-based treatment, many interview participants (especially police and MOLISA professionals) thought that compulsory treatment centres are an effective method for control and treatment. Importantly, they argued that the centres contribute to reducing the crime rate and ensuring social order and safety while protecting the wider community from people who use drugs. Nevertheless, there were many criticisms associated with the existence of the 06 system. Many interviewees blamed the centres for being ineffective and costly, with high relapse rates, poor clinical care and minimal treatment services. Vocational training offered was deemed irrelevant to the labour market. For some interviewees, the centres are maintained because of the wellbeing of centre staff and economic incentives for centre leaders.

This chapter further cements the fact that responses to drug use, addiction and people who use drugs tend to emphasise individual medical solutions, generally ignoring the complexities of drug use behaviours and the intersections between drug use and social or environmental factors. Similar to global abstinence-based responses to drug use, Vietnam has sought to reduce harm by promoting supply and demand interventions and by controlling specific social groups rather than finding rational and effective solutions for drug use problems. Nevertheless, not everyone agreed with these perspectives and practices. Some interviewees considered psychological and social factors in treatment. They also disagreed with a one-sizefits-all approach that isolates people who use drugs from society. Importantly, many agreed with allowing people who use drugs to decide their treatment. Although these perspectives are in the minority, they are significant, as they can pave the way for promoting authentic harm reduction strategies in Vietnam. The next chapter considers how harm reduction is perceived and implemented in Vietnam. It further confirms that fundamental misperceptions in the interpretation of harm reduction has led to a series of misleading interventions in practice. 


\section{Chapter Nine: Findings on Harm Reduction in Vietnam}

\section{Introduction}

In the previous chapter, some study participants engaged with progressive harm reduction principles. This chapter further considers how harm reduction is defined and perceived in Vietnam in order to answer the key research questioned highlighted in Chapter One. Generally, most international and public health workers regarded drug use as a health issue while others (mainly police and MOLISA professionals) viewed it as a social evil. These views impact on how harm reduction interventions are seen. Most participants supported medical interventions (for problematic perceived people who use drugs), such as substitution treatment, sterile needles, syringe distribution and naloxone (overdose prevention medicine). However, other global harm reduction interventions like providing clean injectable drugs or opening drug consumption rooms encountered considerable opposition.

The findings demonstrate that professionals' understanding about harm reduction is narrow because most simply perceived harm reduction as a means to reduce HIV transmission and to treat addiction. As abstinence approaches remain strongly influential, many professionals believed that harm reduction just means reducing supply and demand. Police officers, in particular, were confused as to what harm reduction comprises and many thought that it involves reducing supply. Some local authorities, police and social workers remained sceptical of the benefits of harm reduction and viewed it as fostering and condoning drug use. They also often viewed that sending people who use drugs to compulsory treatment centres is an effective harm reduction method. These perceptions generally show that Vietnam has implemented 'pseudo' harm reduction or harm reduction through an abstinence paradigm.

This chapter records considerable barriers to an 'authentic' harm reduction approach, including insufficient finance, limited understandings about harm reduction, legal discrepancies, and social-cultural factors including stigma and discrimination. Many respondents thought that these barriers can be overcome with government action. Some subsequently recommended possible solutions, including scaling down compulsory treatment centres and allocating centre funding to harm reduction initiatives. Some suggested that Vietnam needs to enhance collaboration among key agencies, improve human 
resources and, importantly, consider people who use drugs' rights, including addressing their social-economic disadvantages. These barriers and suggestions contribute to answering the secondary research questions noted in Chapter One.

\section{Myths about Harm Reduction}

Study participants often misunderstood the harm reduction philosophy. Harm reduction principles, highlighted in Chapter Three, do not demand the reduction of drug use, and are not focused on supply reduction measures. While there was a common belief that harm reduction encourages drug use, there has been evidence to show no increase in drug use (Collins et al., 2012; Marlatt \& Witkiewitz, 2010). Although Vietnam has implemented some harm reduction interventions, like needle exchanges or methadone substitutions since the 2000s, they are a narrow version of harm reduction. In many ways, the difficulties of expanding authentic harm reduction have resulted from a misrepresentation of harm reduction principles in Vietnam. Myths not only came from police and social workers, but also existed among doctors, governmental officials, and even harm reduction officials.

The first common myth among interviewees was the thought that reducing drug intake or demand reduction are harm reduction. Some police even confused preventing drug supply with harm reduction. For them, if supply is controlled, the harm associated with drug use can be mitigated. As previously discussed, harm reduction does not necessarily mean reducing drug use. Yet some interviewees, even doctors and harm reduction workers, believed that reducing the use and the supply of drugs is harm reduction:

Harm reduction means that people who use drugs reduce their drug intake on [a] daily basis or reduce their demand for drugs (Doctor 6).

Harm reduction is that we guide people who use drugs on how to reduce their doses, it is the best if they can give up drugs (Harm reduction worker 1).

Drugs are harmful substances ... harm reduction is to rule out all places that produce and provide drugs (Police 4).

Another common myth was that many interviewees thought that sending people who use drugs to 06 centres is a form of harm reduction. Forcibly sending people who use drugs to 
compulsory treatment goes against an authentic harm reduction philosophy, which establishes that users should seek and receive treatment on a voluntary basis, not through coercion. Yet some interviewees argued that these centres bring benefits not only to people who use drugs, but to society. They said that crime would reduce and social order would be ensured with 06 centres:

06 centres reduce harm and potential risks from people who use drugs to the community, who often break laws (Social worker 1 ).

Sending people who use drugs to the centres would gradually reduce harm for social order and security ... when people who use drugs are sent to the centre, crimes can reduce in society (Government official 2).

Another mythical perception was that harm reduction promotes drug use. As previously discussed, harm reduction is designed to reduce the risks and harms caused by drug use, and importantly it helps users to take drugs in a safer way. It does not foster drug consumption. Yet participants expressed fear that harm reduction measures send the wrong message and cause undesirable consequences:

The locals think these harm reduction programmes foster drug use. Provided needles generally create an offensive image that affects our city reputation ... More importantly, we admit and accept drug use behaviour ... Especially, local people and old people tend to be against harm reduction because they think harm reduction is to 'foster drug use behaviour' and 'the authority is a pander to people who use drugs' (Local police 1).

It [harm reduction] can enable drug addicts to publicly use drugs and use more drugs, and lure more people into drug use and addiction ... it can reduce harm to addicts in the short-term, but negatively affect their families and citizens (MOLISA official 3).

Notably, a few police argued that harm reduction is seen as 'an amulet' that allows people who use drugs to take drugs without worrying about being arrested: 
People who use drugs think that joining methadone programmes will be an 'amulet' or 'Kim Bai Mien $\mathrm{Tu}^{\text {'76 }}$ that permits them to use drugs without receiving punishment ... if we are not strictly controlling them, they will use drugs again (Police 3).

When they have [a] methadone card they will not be sent to treatment centres, it means they can still use drugs ... when we arrest them they are going to show the card ... so we have to release them (Police trainer).

Overall, abstinence was mistakenly considered a component of harm reduction by many participants, especially police, MOLISA professionals and local leaders. Harm reduction, for them, refers to any measure that can reduce drug use and drug crimes. These misleading perceptions are not only due to a lack of understanding about harm reduction, but more to perceiving the term 'harm' within a Vietnamese context. As noted previously, from a Vietnamese perspective, harms caused by drug use are solely attributed to drugs and people who use drugs, so harm reduction refers to any intervention that reduces drug use (Vuong et al., 2016). These misperceptions have influenced participants' perceptions and attitudes towards harm reduction philosophy and interventions.

\section{Understanding Harm Reduction}

Almost all participants were able to provide their own definition of harm reduction. While the majority of interviewees viewed harm reduction from a narrow public health perspective, a few also engaged with wider social perspectives (considering social, environmental, and psychological factors).

\section{Public-health Perspectives}

Most participants defined harm reduction as a tool for HIV prevention and addiction treatment. As a result, for most people, harm reduction is the main method for addressing HIV and drug addiction for problematic drug users:

\footnotetext{
76 In Vietnamese culture and history, 'Kim Bai Mien Tu' is a card made from wood or pottery, meaning that if someone holds this card, they will not be subject to punishment for whatever they do. This card is issued by the king, so it is a powerful tool that everyone has to follow and obey.
} 
Harm reduction is to reduce harm caused by risky behaviour in order to mitigate HIV transmission ... it can help those who are in risky groups to have a better health and try to quit their risky behaviour ... by doing this it can contribute to reducing HIV infection (Local authority).

Harm reduction is a health-based intervention to reduce harm caused by drugs ... harm reduction is the most powerful tool in preventing HIV ... harm reduction is to treat addiction or relapse (Doctor 7).

The majority of participants had a positive view about harm reduction from a health perspective. For example, some interviewees explained:

It helps people who use drugs to improve their health and quality of life ... contributing to precluding HIV transmission and blood-borne diseases like Hepatitis B and C or other social diseases because patients no longer share needles and syringes (Government official 1).

Taking methadone, they [people who use drugs] don't have demand for other drugs, overdose problems are therefore mitigated, especially among drug injecting groups ... further, in harm reduction clinics, they are not only provided with medicines, but they also get psychological consultations (Consultant).

It can be seen that harm reduction remains firmly rooted in a narrow public health perspective among interviewees. This is understandable because, as noted previously, Vietnam has widely implemented harm reduction programmes related to addressing HIV infection and drug addiction. Nevertheless, some respondents took a wider social perspective of harm reduction, which bodes well for the development of harm reduction in Vietnam.

\section{Social Perspectives}

Harm reduction was highlighted, by some, as an effective approach to ensure social and public order. Interviewees explained how harm reduction helps to ensure public safety and reduce crime:

... The cops realised they're not stealing things any more, they're being good members of the community, I don't have to worry about that guy any more ... The family's happy 
because they're [users] getting their medicine and they're not doing all the bad things they used to do before in their mind (International worker 1).

They [people who use drugs] don't need to get money by committing crimes like robbery or theft because they can use methadone instead ... social and public order also get better as a result (Harm reduction worker 2).

Some interviewees saw that harm reduction connected people who use drugs with their families, society and supportive services. That is, harm reduction strategies operate as a bridge that allows people who use drugs to access support services from society and the government:

It increases social cohesion between people who use drugs and society, we can see them every day and encourage them to come to medical clinics for treatment ... we can manage them better and have closer connections with their families. We also have opportunities to offer them vocational training, jobs, and programmes of community re-integration (Doctor 4).

Their [drug user's] relationship with family and other people improves ... they no longer antagonise others because of their deviant behaviour, their family also less suffers the displeasure caused by people who use drugs ... their relationships with police and local authorities are also better (Harm reduction worker 1).

Notably, about a third of interview participants ( $n=$ ?), police included, highlighted the benefits of harm reduction to help people who use drugs to integrate into their community and prevent stigma and discrimination towards users. For example:

Harm reduction helps minimise stigma and discrimination. I don't necessarily think, I know it doesn't promote drug use. And, in fact, it may help to promote drug treatment (International worker 2).

Harm reduction helps to increase people's knowledge and understanding of addiction and promotes reducing stigma and discrimination (MOLISA trainer). 
People who use drugs will not be discriminated [against] because, for methadone users, only police, doctors and MOLISA staff know ... they [methadone patients] still work and live like a normal person. Harm reduction does not isolate people from their community ... they are not a burden on society (Police 6).

In general, many interviewees perceived harm reduction as an effective method to ensure public safety, connect people who use drugs with health and social services, and contribute to preventing stigma and discrimination. These perspectives are significant because they demonstrate space for authentic harm reduction in a Vietnamese context that currently prioritises punitive-based approaches. While interviewees often appreciated harm reduction, none mentioned the importance of promoting people who use drugs' rights, which is an essential component of harm reduction philosophy. Also, no interviewees challenged the deficiencies of prohibition and abstinence approaches, an issue that will hamper the development of authentic harm reduction in a Vietnamese context.

\section{Attitudes towards Practical Interventions of Harm Reduction}

Vietnam has pursued harm reduction interventions since the $2000 \mathrm{~s}$, including the provision of substitute substances (methadone and buprenorphine), sterile needles and syringes, and naloxone (integrated into methadone clinics). However, other harm reduction interventions, such as clean injectable drugs or drug consumption rooms, have not been available. Asking study participants about these unavailable harm reduction approaches is important to understand whether or not harm reduction can go further in Vietnam. There were diverse attitudes towards these practical harm reduction interventions.

\section{Substitution Treatment}

The overwhelming number of interviewees $(n=25 / 26)$ agreed that substitution treatment (Methadone) should be promoted. Although, as noted previously, methadone is highly addictive, the majority thought that methadone is less harmful than other drugs, can treat addiction and contribute to ensuring social order and security: 
Methadone is an effective method in addressing drug addiction, it is a substitute substance that is less harmful than other drugs like heroin. This drug can help people who use drugs for a long period of time (Police 4).

Similarly, the majority of survey respondents (over $69 \%, n=177$ ) believed that methadone is effective treatment for people who use drugs (see Figure 9.1).

Figure 9.1: Oral methadone is effective treatment for people who use drugs

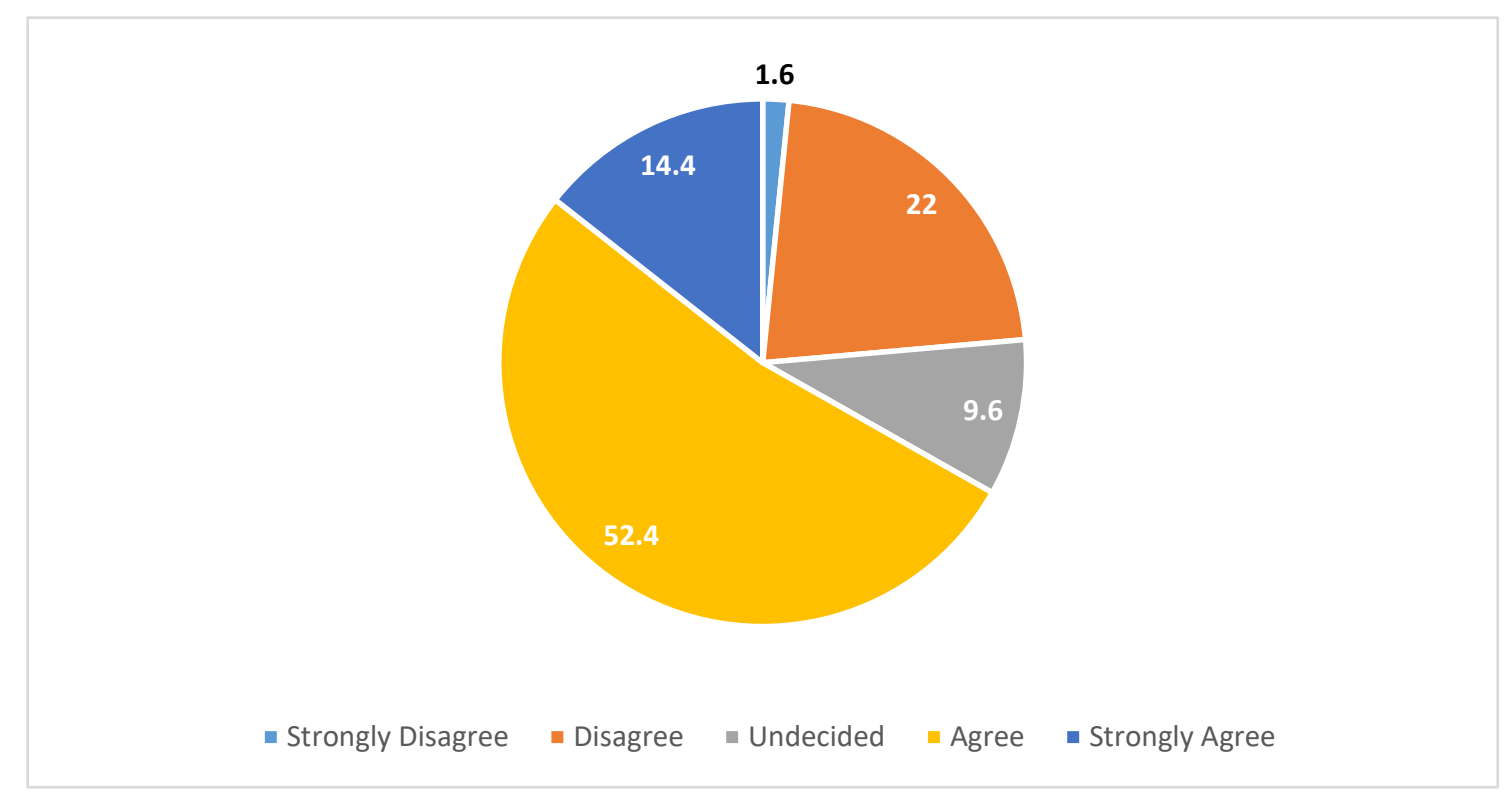

Interestingly, one interviewee who works within the social work sector criticised methadone, arguing that people tend to overestimate its value:

We are overestimating methadone, and seeing it as a golden method to address addiction. We should know that many countries do not allow methadone, like Indonesia ... I don't think we should provide people who use drugs, who are young and just used drugs, with methadone ... they need treatment rather than methadone ... because they can be addicted to methadone (Social worker 3 ).

His arguments are similar to previously presented concerns about methadone (it is addictive and has severe side-effects, leading to users' deaths in some cases) (Corkery, Schifano, Ghodse, \& Oyefeso, 2004). Nevertheless, methadone was supported by the majority because, as noted in Chapter Five, Vietnam regards methadone alongside needle distribution programmes as one of the two fundamental harm reduction interventions in the country 
(Vuong et al., 2016). The effectiveness of methadone maintenance in reducing illicit opioid use, morbidity and death from overdose, and decreasing people who use drugs' involvement in crime as well as HIV risk behaviours, is evident in many countries (Ducan et al., 2014; Kermode et al., 2011; Wodak, 2011), including Vietnam (Hoang et al., 2015; Reid \& Higgs, 2011; Tran, Ohinmaa, Duong, Do, Nguyen, Nguyen, et al., 2012; VAAC, 2011). Yet acknowledging its potential risks and drawbacks is also essential for its proper implementation.

\section{Sterile needles and Syringes}

The majority of interview participants $(n=22 / 26)$ favoured providing sterile needles and syringes to people who use drugs to prevent blood-borne diseases. For example:

Needle and syringe programmes prevent the transmission of HIV, Hepatitis C ... At the same time, if it's an exchange programme, then the dirty needle is actually collected and canned at hand, which means it's not lying around in the neighbourhood and the community for someone else to pick it up and hurt themselves with (International worker 2).

Similarly, the majority ( $n=211 / 250$ ) of survey respondents believed that people who use drugs should be provided with sterile needles and syringes (see Figure 9.2).

Figure 9.2: People who use drugs should be provided with sterile needles and syringes

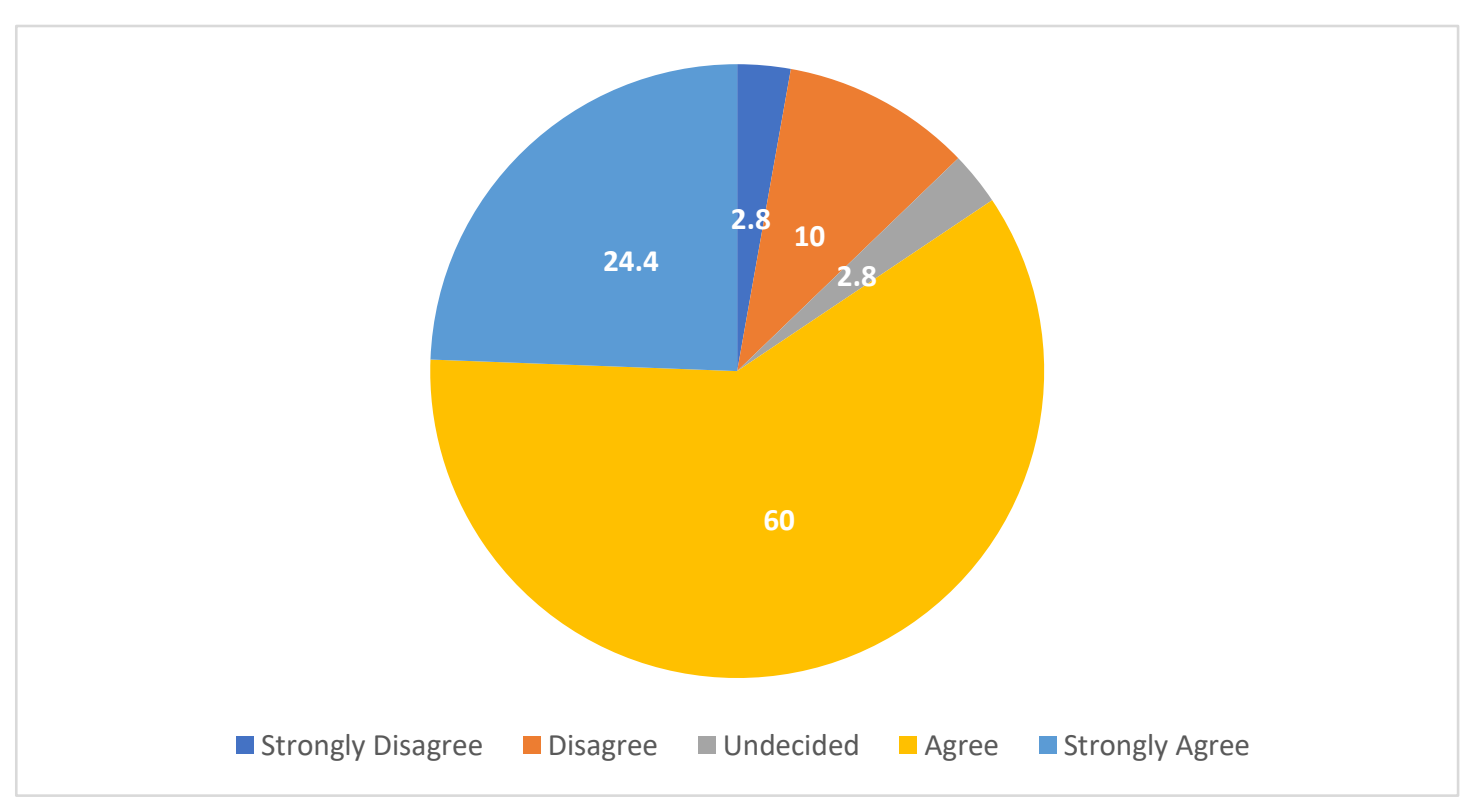


However, some police had quite negative views on needle exchange programmes, viewing them as legitimising drug use. Officers thought that the programmes fail to condemn drug use, and that they actually foster drug use:

We are preventing drug use ... so distributing sterile needles and syringes is like we are accepting drug use behaviour (Police 5).

In short, an overwhelming number of respondents supported needle and syringe exchanges. Yet some police were sceptical because they thought the programmes encourage drug use. These results reflect previous studies (See Edington \& Bayer, 2013; Hammett et al., 2008; Khoi Do, Victor Minichiello, \& Hussain, 2012; Khuat et al., 2012). Therefore, scaling up needle and syringe exchanges is still challenging.

\section{Naloxone}

Most study participants supported providing people who use drugs with naloxone to counteract the effect of overdose and save their lives. The survey data show that over $50 \%$ of respondents ( $n=138 / 250)$ favoured naloxone (see Figure 9.3).

Figure 9.3: People who use drugs should be provided with naloxone (an opioid antidote) to reduce fatal overdoses

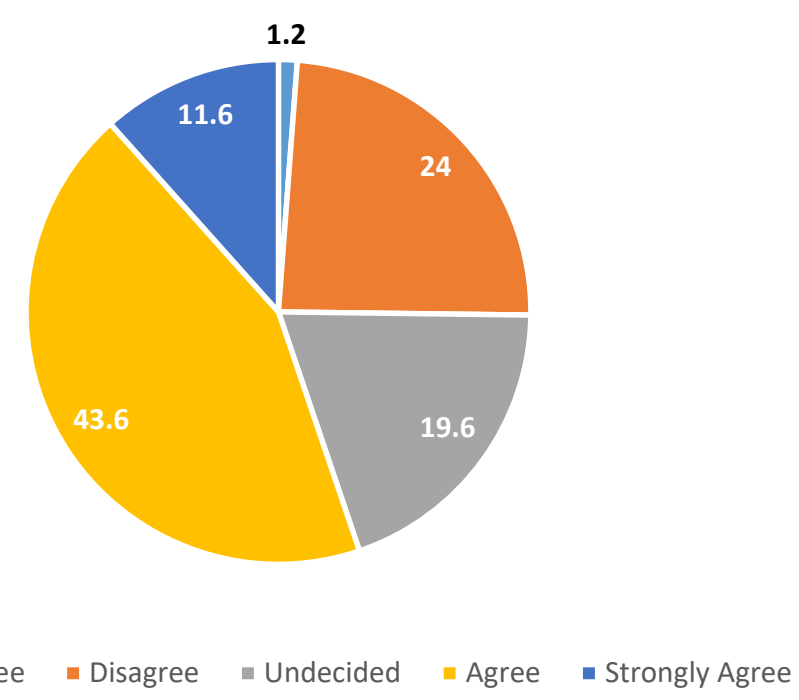


Meanwhile, except for a few police and social workers, almost all interview participants supported this medication for people who use drugs. Those against it argued that if naloxone is provided, people who use drugs will increase their drug intake:

People who use drugs will subjectively think that 'it doesn't matter because we have medicine available if they overdose', so they continue to use drugs (Social worker 3).

Providing naloxone to addicts will encourage them to increase their daily dose, which is more detrimental (Police 2).

However, many interviewees asserted that naloxone is necessary to save people who use drugs' lives. They suggested that the drug should be provided not only to doctors, but to others such as police, political and social organisations, social workers or even sold in pharmacies. For them, saving lives is vital:

When they [people who use drugs] face overdose, we can provide this drug to them or we can sell it or we can inform them about this drug, so that they can come over to buy it (Social worker 5).

Another harm reduction strategy that often isn't really thought about is the dissemination or use of naloxone [drug] and reducing overdose deaths. That's also a benefit. In my mind there isn't any detriment or negative side to training peers or training other professionals including the police how to administer naloxone should they come across someone (International worker 2).

Although naloxone has gained support in the public health sectors, some participants misunderstood its benefits. They thought that naloxone is used to treat addiction and to reduce drug use. This misunderstanding is apparent through all work sectors, including harm reduction officials. For instance:

Naloxone is a solution to give up drugs, and it is abstinence based. To take naloxone, a drug user needs to quit drugs or they need to gradually reduce their doses ... If they use naloxone, they can face overdose. Naloxone is also a supplementary substance to treat addiction (Social worker trainer). 
This drug is an opioid antagonist ... when people who use drugs take it they will forget drugs ... because it helps to detoxify heroin (Harm reduction worker 2 ).

Similarly, 49 survey respondents had no idea about naloxone, accounting for nearly $20 \%$ of the survey sample. As noted in Chapter Five, naloxone is quite new to Vietnam but some overdose prevention programmes (using naloxone) have recently been integrated into methadone clinics across the country (Ministry of Health, 2016b).

In summary, naloxone received strong support from the majority of both survey and interview respondents, despite some misunderstanding about its uses. As reported by Family Health International $(\mathrm{FHI})$, insufficient training is a key reason that hampers the development of overdose prevention programmes in the country (Hoang et al., 2015). Therefore, the lack of knowledge and perceptions of naloxone is understandable. Yet Vietnam has adopted naloxone as a nationwide harm reduction intervention, and it has become an important part of public health work to assist people who use drugs. Furthermore, providing clean injectable drugs for heavily addicted users and opening drug consumption sites still faces many challenges. 


\section{Clean Injectable Drugs}

Interviewees and survey respondents had similar views on whether doctors should be permitted to prescribe injectable opioids (mainly heroin) for those with heavy drug dependence. Just over $28 \%(n=71)$ of the survey respondents agreed or strongly agreed with this approach, while only a few interviewees have considered it. The reasons for not providing clean injectable drugs to people who use drugs were that 'all drugs are harmful', such drugs can 'promote drug use', or they imply 'accepting drug use':

We should not provide such drugs to addicts for sure because if provided drugs are cheaper than drugs from black markets it will promote and foster drug use. Besides, I don't think we have clean drugs because all drugs are harmful and make users become dependent on them ... by providing this drug we admit and accept drug use behaviour, which is banned in the drug and criminal law (Police 1).

The more you take drugs the more harm you will suffer ... they cannot quit drugs, doses of using drugs will increase ... they will no longer have a future ... they will go mad or it will lead to suicide or cause harm to their family and society (Doctor 6).

It seems if we accept drug use, people will take drugs publicly and widely ... it will lure more people into drug addiction ... social disorder will be affected ... it can be less harmful to drug addicts, but it will have negative impacts on their family and their neighbours (MOLISA official 3).

Ironically, methadone is also a drug but it is accepted in Vietnam. As noted previously, inspired by the UN conventions on drugs, Vietnam considers some drugs legal, while it views others as illegal drugs. The Vietnamese drug classification is based on social and political constructions rather than scientific evidence. The views of those participants generally ignore the motivations for drug use and the myriad reasons why people use drugs problematically. Their views are understandable, given that myths about the nature of drugs, drug use and addiction are prevalent in Vietnam, as discussed in Chapter Seven. 
Other participants, mainly those working for international sectors or harm reduction sectors, expressed that such drugs could be possibly used, but they need time to be accepted because of Vietnamese culture, custom and legal frameworks. For instance, some remarked:

If the state can produce and regulate such drugs, this will be fine ... or if our government views those drugs as methadone, it will be ok (Doctor 1).

I think it probably could work, but I think it would be 70 years before the systems were actually in place and the attitude was changed. Because, as you know, morphine is given medically. It's done the same, it's a derivative of the same base, opium. So it's just when they cook or refine morphine more you get heroin. So it's possible and plausible (International 1).

It is evident that providing or prescribing injectable opioids to treat people with drug dependence is still far from being endorsed, even though the Swiss model has proven the effectiveness of providing clean injectable drugs to heavily addicted users. It would be a challenge, given drug use is seen as a social evil, and Vietnam wants to be a drug-free country. Therefore, convincing Vietnam's government that illicit drugs like heroin, opium or cannabis can be legalised for medical and recreational purposes (like morphine or methadone) would require some time.

\section{Drug-consumption Rooms}

Over three-quarters of interview participants did not favour opening drug-consumption rooms. They mainly thought that such rooms would be a hub where people who use drugs gather, and it would thereby foster drug use and undermine addiction treatment. They explained:

I don't like this room, because I see it as very offensive, drug addicts come to the room and gather and inject drugs, and then lie on and sleep on the floor ... they are careless and reckless, just inject drugs and throw away needles and syringes everywhere (Doctor 2). 
This room is not a long-term solution because our goal is to help addicts to quit drugs. Now, if we provide such a room where they can come to take drugs, their addiction will remain unchanged (Police 3).

Their views reflect myths about consumption rooms, which are demonstrated as being effective and providing people who use drugs with many benefits (this will be further discussed later).

The survey finding is interesting. About $43 \%(n=109)$ of the survey respondents supported drug-consumption rooms, compared to around $46 \%(n=110)$ of those did not (see Figure 9.4). This finding is optimistic, given the prevalence of punitive approaches in Vietnamese drug policy.

Figure 9.4: Medically supervised rooms (drug-consumption rooms) should be available

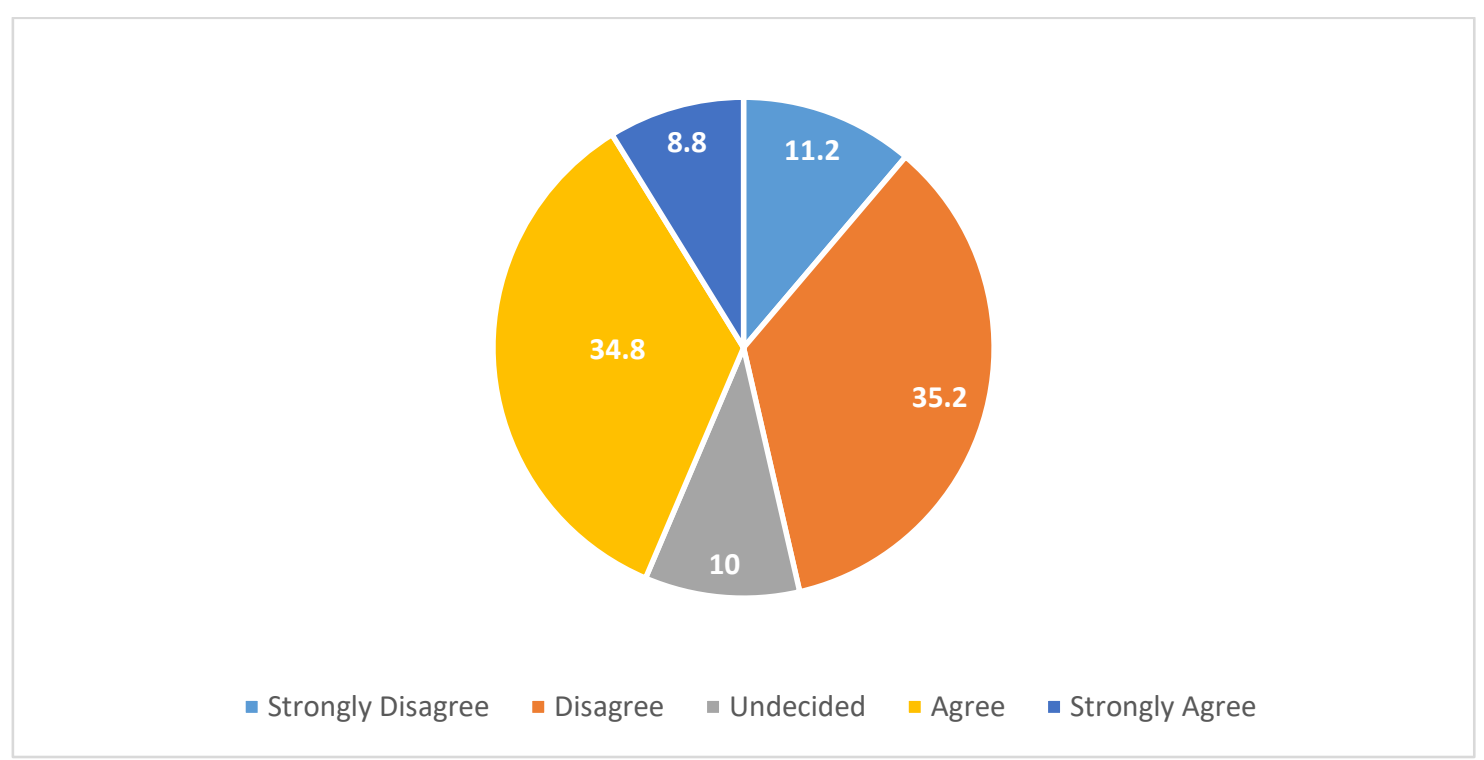

In addition, some international interviewees held optimistic views on drug-consumption rooms because they believed that people who use drugs would have a safer place to consume drugs. This would prevent overdoses, but also create a bridge to connect people who use drugs with supportive services:

We will provide people who use drugs with sterile needles and syringes or first aid ... they don't need to conceal themselves ... they can have access to safe injecting equipment (International worker 6). 
I would want a safe injecting room in which it could be used also as a point of contact with the system ... So it's a place where you can go and safely inject ... but it's also a place where you can get connected, if you want, to other services, not necessarily provided at that location, but referred to testing, counselling, or referred to a methadone programme (International worker 1).

In general, the majority of interviewees (over three quarters) did not support opening consumption rooms and felt that the rooms would face strong opposition from the local community. To date, there are no drug-consumption rooms in Asia (International Harm Reduction Association, 2016). Nevertheless, 88 drug-consumption rooms (DCRs) have been implemented in ten countries, mainly in Europe (International Harm Reduction Association, 2016). These facilities allow people who use drugs to bring injectable drugs and to legally inject or inhale drugs in a user-friendly, medically supervised space. Importantly these have been very effective in reducing overdoses, and increasing use of health and social services, as well as minimising risks associated with drug use (Wood et al., 2006). Although drugconsumption rooms have many demonstrated benefits, they would face resistance in Vietnam, given contemporary mind-sets towards drug use. There remain significant barriers to authentic harm reduction in Vietnam.

\section{Barriers to Implementing 'Authentic' Harm Reduction}

The findings show that there was a considerable diversity of opinion in relation to harm reduction related barriers, contributing to answer the research question on how problematic perceptions impact on harm reduction in Vietnam. The main barriers identified by interviewees include: (i) insufficient financial resources, (ii) limited understanding of harm reduction, (iii) social and cultural factors, (iv) discrepancies in relevant laws, (v) administrative barriers, and (vi) stigma and discrimination.

\section{Insufficient Financial Resources}

About two-thirds of interviewees identified the lack of financial resources as a key barrier to facilitate harm reduction interventions in Vietnam. As noted previously, Vietnam's emergence as a middle-income country in $\mathbf{2 0 1 0}$ has led to a significant decline in international 
donor funding towards Vietnam's harm reduction efforts. Many participants expressed that continued underfunding is a major impediment:

The biggest difficulty for Vietnam is money ... In fact, we are so poor, if we open services, we need staff ... where we will get money to pay for managing harm reduction services? (Governmental official 1).

At the moment, the biggest barrier is not legal aspects, obstructions of police or limited understanding of citizens, actually it is money. Beforehand we used international aid to buy needles, syringes and methadone, but now external funding is significantly cut ... when the government balances the national budget, they tend to prioritise medicines to treat HIV (International worker 3).

As a result of underfunding for harm reduction, a few participants expressed their concern over limited human resources. The lack of skilled practitioners at local levels is seen as a stumbling block for scaling up harm reduction networks:

Human resources are insufficient and unskilled. For example, as a consultant, I find myself incapable of meeting over 300 patients' needs ... so it is hard to ensure the quality of consultation (Consultant).

Vietnam set up the target with over 80,000 addicts using methadone services, but our human resources are not enough. Many addicts abandon methadone, and many use other drugs while they are in methadone treatment ... because methadone is not really accessible and does not bring a good feeling like other drugs (Social worker trainer).

Given underfunding, many interviewees acknowledged that Vietnam has failed to expand harm reduction interventions to local levels. They argued that many services are far away from residential areas, and coverage is patchy at local levels. People who use drugs have to commute long distances to access clinics, which significantly affects their work and lives:

There is a system from the central to local levels, but it is not widespread enough ... each district has one methadone unit ... many people who use drugs have to commute 
around $20 \mathrm{~km}$ just to get one dose of methadone and go to work afterwards ... it is hard for them to maintain and follow the treatment (MOLISA official 6).

Quotas on the number of people who use drugs who can have access to harm reduction are very low compared to demand ... many people wanted to join ... for example I live in Binh Thanh District, so I go to Binh Thanh District Clinic, they said the clinic is full, then I go to another district, but they said why you not get methadone at Binh Thanh district ... where should I go? (International worker 4).

Financial issues were also raised by most interviewees as a key barrier to developing harm reduction services in Vietnam. Insufficient funding has led to a lack of resources to train and develop workers in harm reduction measures or to expand harm reduction services to local levels. This generally hampers the engagement of people who use drugs with the service. Yet as previously argued, underfunding is not the only barrier for promoting harm reduction, as limited knowledge also hinders its development.

\section{Limited Understanding of 'Authentic' Harm Reduction}

Interviewees frequently highlighted that harm reduction does not work well at local levels because it is not properly understood. They stressed that locals think that harm reduction fosters drug use:

Elderly people at local levels who have a socially strong influence are opposed to harm reduction ... they said it's like 'showing the path for the deer to run away' (Harm reduction official 2).

It's a general population lack of understanding of harm reduction, lack of understanding of drug use, lack of understanding of drug treatment and what is addiction ... And the reason why I say a lack of all that, is because when they don't have that education, or that understanding, they like to stick with what they know. And what they know hasn't worked, and what they know isn't evidence-based ... So they don't see it as a public health issue, they see it as a moral problem (International worker 1). 
In Vietnam, harm reduction is seen as promoting drug use and therefore people tend to have negative attitudes toward it (Edington \& Bayer, 2013). Some participants shared that harm reduction knowledge is still generally provided to professionals rather than local people and authorities, making it hard to implement at local levels, and also hard to help local/ordinary people understand authentic harm reduction. Judgemental attitudes towards drug use and people who use drugs make it harder to implement harm reduction. This challenge can be explained by some typical social and cultural factors within Vietnamese society.

\section{Social and Cultural Factors}

Some interviewees argued that social-cultural factors like the 'cult of results' (emphasising reputation and appearance) hamper the development of authentic harm reduction. For example:

Our barrier is 'cult of results'. When local authorities announce that 'my jurisdiction doesn't have drugs' ... people who use drugs want to attend harm reduction services, so they come to register ... but police and local governments say ' $\mathrm{No}^{\prime}$, because they already registered for 'No people who use drugs' at their wards (Doctor 1).

Another interviewee explained that Vietnam still follows an approach based on 'cure rather than prevention'. Therefore, professionals mainly deal with urgent problems rather than longterm solutions:

A Vietnamese ideology is that 'we don't need firefighting until the house burns' or 'don't lock the stable door after the horse has been stolen'. So now we don't have money to buy needles and syringes, these things are not prioritised by the local budget ... harm reduction does not have money, doing treatment is easier to receive funding. For example, if we give medicines to patients, and they then get better, we have a better reputation than distributing needles and syringes to prevent HIV, which will not draw much attention (International worker 2).

In line with these approaches, it is not easy to reach a consensus when implementing harm reduction at local levels. Many interviewees expressed their concern that the imperial power bends to suit rural customs' in Vietnamese culture. This means that, although harm reduction 
has been adopted and regulated in the 2006 HIV Prevention Law, it is still not straightforward to implement at local levels:

It is easy to convince leaders from the central levels, but it is extremely hard to do so at local levels ... because when a local leader has an opposite opinion, the programmes are impossible to implement (Harm reduction official 3).

We need to achieve a consensus between local authorities and the community, because many people said that 'imperial power bends to suit rural customs' ... although we have legal framework, the local authorities announce that their jurisdiction don't have drugs, so distributing needles and syringes is not allowed ... we also rely upon the local budget to operate harm reduction ... if they don't advocate for it, harm reduction will be hard to implement (Harm reduction official 1).

These social-cultural factors add a new dimension relating to barriers to harm reduction in Vietnam. Reputation concerns are significant in Vietnamese culture. Vietnam has a social movement called 'Khu Pho Van Hoa' or 'Khu Dan Cu Van Hoa'. ${ }^{77}$ These social movements explain why local authorities do not want to have harm reduction services in their jurisdiction, because this means that their area has people who use drugs. Also, local authorities are unwilling to spend money on harm reduction, because helping people who use drugs is not as urgent and important as treating other diseases. Although Vietnam has some legal frameworks for implementing some medical interventions in the name of harm reduction, social-cultural factors like the dominance of local leaders reduce the possibilities for authentic harm reduction at local levels.

\section{Discrepancies in Relevant Laws}

Another barrier, as previously discussed, is the contradiction in carrying out the HIV law, the drug control law, the administrative law, and other decrees regulating drug treatment. The

\footnotetext{
77 A major social movement in Vietnam at local levels, imposing many requirements related to infrastructure, education, poverty levels, or no law violations. No local members involved in drug use is a key criterion.
} 
rigidity in law enforcement generally results in blocks to implement harm reduction in practice:

There are three legal handcuffs that are on addicts' necks. We are conducting harm reduction according to the HIV law ... addicts have to attend compulsory treatment regulated by the drug control law and administrative law ... there are no clear regulations regarding who will go to compulsory treatment centres and who will participate in methadone programmes or substitution treatment (Harm reduction official 5).

There is a disconnect between the drug law of Vietnam and the HIV law of Vietnam. And that's a problem, because while one says one thing, the other one says something else. And you listen as a policeman to the drug law, not necessarily the HIV law ... the policies currently in place prohibit people from coming forward, people from accessing care ... confidentiality and privacy don't exist in Vietnam (International worker 2).

The contradictions between relevant laws have also led to conflict regarding the implementation of harm reduction in practice, according to some interviewees:

I see complexities between law enforcement sectors and public health sectors when it comes to drug testing ... in many cases, relevant agencies already allowed people who use drugs to attend treatment, but police still ask them for medical testing (Consultant).

Public health sectors distribute free sterile needles and syringes, but police give chase and confiscate them ... today we distribute needles and syringes to 10 persons, but the day after all of them are arrested, so they no longer believe in service providers ... today clinic open, tomorrow police come and ask for test results and patients are arrested, so no one is willing to come [to] clinic anymore (International worker 3).

The inconsistencies in law and policy have resulted in significant confusion between practitioners, especially among police (Ahmed et al., 2014). Importantly, these inconsistencies in law and policy mean that people who use drugs distrust service providers, 
because of the high risk of being arrested and being sent to compulsory treatment centres. Further, even when a drug user is willing to join services, which ask for many requirements to be a member, they face administrative barriers. These administrative barriers go against a low-threshold approach of authentic harm reduction measures.

\section{Administrative Barriers}

Interviewees highlighted administrative barriers that hamper people who use drugs' access to harm reduction services. People who use drugs have to disclose themselves, and they need to come to local authorities and ask for a confirmation/introduction letter to participate in services:

The community had to know that I was an addict ... But let's say I wanted to go to the programme. You would have to have a letter from the local district people, local authority, to say okay ... Then I get on the master list of people, then I have to get medically cleared. After I'm medically cleared, then I have to go then this council, I call it a council, sits to decide, of this list of 20 , who gets to go (International worker 1 ).

In order to get into services, they need the confirmation from local governments, this by and large will rule them out of the services because they are scared to see police, but they need to see police to get the confirmation. That is a big barrier (International worker 4).

If those who wanted to join programmes, they would need to get through many procedures for considering and approving ... undergoing many steps with the involvement of police and local authorities (Government official 1).

Another dilemma is that local harm reduction clinics are mainly served and allocated to local people who use drugs, meaning that if you are from another locality you will generally not be allowed to use the services:

As for patients who are from other districts, they are hard to engage in treatment, because district clinics prioritise patients who live in their jurisdiction. I am not sure this is due to orders of district leaders or not, but it restricts people who use drugs from joining services (Consultant). 
Administrative requirements have made it harder for people who use drugs to access harm reduction services, and they face high levels of social stigma and discrimination in doing so. This conflicts with harm reduction principles that make few demands upon users. Harm reduction needs to be user-friendly, accessible, and open. It requires a high level of flexibility and tolerance to accommodate users (Buchanan, 2015b; Collins et al., 2012). Instead, Vietnamese people who use drugs encounter intense social stigma and discrimination, as well as administrative and bureaucratic barriers.

\section{Stigma and Discrimination}

The majority of participants identified that stigma and discrimination against those attending harm reduction services are widespread. People who use drugs are labelled as persons who fail to quit drugs, who have lost control and have moral problems or a disability. This partly explains why many people who use drugs feel unwilling to engage in harm reduction interventions. For example, some explained:

People don't care about moral status and health status of patients, if anyone comes to the clinic to get methadone, they will be subjected to be stigmatisation (Doctor 6).

They are not willing to disclose themselves and they will not appear in harm reduction clinics ... they are always fearful of being discriminated against, and they are scared to be arrested as well (Social worker 1 ).

Stigma and discrimination related to those infected with HIV/AIDS are serious. This makes many people who use drugs unwilling to access HIV testing, sterile needles and syringes or methadone ... this limits the efficiency of harm reduction (Local authority).

I go to the programmes, then maybe I become on someone's list, and they know about me, and the next thing you know, the local person's coming to my house to see what's going on ... and if I get known I'm subject to stigma and discrimination (International worker 1).

The high levels of stigma and discrimination within Vietnamese culture have served as a major hindrance for people who use drugs to access harm reduction services. People who use drugs are widely perceived as those who undermine Vietnamese moral traditions, they are seen to 
have pathological failings, and they are blamed for their drug-use behaviours. These judgemental attitudes make it difficult to implement authentic harm reduction measures in Vietnam.

In summary, a wide range of barriers hinders the expansion and development of harm reduction, especially in engaging more people who use drugs into the services. Underfunding means that services and training for workers are significantly limited. However, the key problems are related to the legal framework and common perceptions towards harm reduction and people who use drugs. Both criminal justice and social sanctions have put people who use drugs under pressure and they face stigma and discrimination when they want to join harm reduction interventions. The next section illustrates the solutions proposed by research participants to address drug use related problems and to promote authentic harm reduction in Vietnam.

\section{Moving Forward}

After identifying the main problems of Vietnam's drug policy and the barriers to harm reduction, participants were asked how Vietnam should develop drug policy, services and programmes. They gave different recommendations to improve the current drug policy, proposing social, legal and political solutions, including: (i) the closure of compulsory

treatment centres, (ii) enhanced cooperation between relevant agencies, (iii) improving human resources, (iv) the reformation of legal frameworks, (v) acknowledging people who use drugs' rights, and, (vi) addressing social and economic disadvantages. Their recommendations helped to answer the research question related to what should be done to facilitate improvements in harm reduction strategies in Vietnam.

\section{The Closure of Compulsory Treatment Centres}

In contrast to police and MOLISA professionals, international and public health interviewees called for the reduction and closure of 06 centres because they are ineffective. For instance, one interviewee stated:

MOLISA needs to change their attitude towards 06 centres, they believe the centres work, so they do not consider research from Ministry of Health ... we have to accept 
that all approaches to drug use need to be based on evidence ... we cannot think that the centres have existed for a long time, so we cannot shut them down ... we need to accept that if the centres are not effective, we have to close them down (International worker 2).

Other interviewees suggested that family and community-based treatment should be promoted in place of compulsory treatment. For example, one interviewee working for the government suggested:

It is better if people who use drugs live in their community ... the community should look after them, not government ... we need to promote the role of community and family ... we should not keep current approaches that prioritise sending people who use drugs to compulsory treatment centres ... treatment also needs to be individualised (Government official 2).

One interviewee explained that if the government closes down about half of the centres, Vietnam will have enough money to maintain and develop harm reduction. They took Ho Chi Minh City as a typical example:

We should reduce the funding allocated to compulsory treatment centres and spend that funding on buying Methadone and supporting harm reduction services ... I think if we scale down about $50 \%$ [of] the number of the centres, we have more than enough money to support harm reduction programmes like methadone treatment or needle exchange. Ho Chi Minh City, for instance, they have 13 centres, if we close half of the centres we will have more than enough money to cover harm reduction services in the city (Harm Reduction official 3).

These opinions on closing and scaling down 06 centres are generally consistent with previous studies (See Edington \& Bayer, 2013; Hoang et al., 2015; Khuat et al., 2012; Reid \& Higgs, 2011; Windle, 2015). These recommendations are not new, because 06 centres have been heavily criticised as being ineffective and having counterproductive effects (Human Rights Watch, 2011; UNAIDS, 2016). Therefore, transforming the current 06 centres is necessary. This could start with improved cooperation among key stakeholders. 


\section{Enhancing Collaboration}

While police and MOLISA professionals suggested enhancing collaboration among relevant sectors to prevent drug use, other interview respondents highlighted that improving collaboration among the sectors would lead to greater harmonisation for health services and harm reduction. For instance, one international professional argued:

Three main sectors, including public health, law enforcement and social work need to closely cooperate ... for example, police not only need to concern about reducing demand and supply, also they need to care about harm reduction because if harm reduction is promoted, supply and demand reduction measures would be supported ... MOLISA should collaborate with Ministry of Health $(\mathrm{MOH})$ and should trust studies of $\mathrm{MOH}$... what $\mathrm{MOH}$ demonstrates effectively should be applied so that people who use drugs have more opportunities to access health and treatment services (International worker 2).

Others believed responsibilities should not be the sole domain of the public health sector $/ \mathrm{MOH}$, and called for greater cooperation from other governmental sectors and private sectors. For instance, one interviewee elaborated:

We need to involve the participation of MOLISA in harm reduction programmes ... if only $\mathrm{MOH}$ implements harm reduction, it is not enough resources to expand services ... we also need to reach a consensus with police when doing harm reduction ... besides we should engage more private sectors and promote the role of society into harm reduction in order to address financial concerns (Harm reduction official 5).

Increased cooperation among relevant agencies is needed. As discussed previously, there have been conflicts and tensions between Police, MOLISA workers and others working for public health and international sectors when it comes to implementing harm reduction. As Vuong et al. (2012) explain, drug addiction treatment and rehabilitation is the responsibility of MOLISA with the support of police, but treatment and care related to HIV and harm reduction lies with $\mathrm{MOH}$. Therefore, inter-sectorial cooperation has been superficial $(\mathrm{Ha}$ et al., 2010), creating challenges for implementing supportive harm reduction programmes. To 
overcome tensions between the sectors, some recommend that Vietnam should reform their drug policy.

\section{Reforming Legal Frameworks}

Interviewees stated that the effectiveness of harm reduction depends largely on how Vietnam reforms current legal frameworks. Some emphasised the importance of having laws for treatment and harm reduction:

Like Thailand, Vietnam should have a law for addiction treatment ... addiction treatment needs to be separated from drug prevention laws. Making these two laws clearer (Doctor 5).

We should have a separate law for harm reduction interventions, especially for people who use drugs ... this law needs to be specific and all local authorities have to follow it ... I think this can create a harmonising environment for conducting harm reduction (Harm reduction official 2).

Others believed that administrative barriers need to be removed to make harm reduction more accessible to people who use drugs. For instance, one international worker recommended:

In the short term they need to get rid of some of these rules that prohibit access, like getting a letter ... if you have a problem, you come to the clinic, the clinic assesses you and medically decides this treatment is appropriate, and then sets you up into the programme (International worker 1).

This worker also suggested that in the long-term drugs should be regulated:

I think there can be some policies that are put in place, such as taxation, and rules about who can buy certain products ... I would say that if we tax the drug then the money should go back into the drug treatment side of the system and not go to buying weapons, or buying other things. 
As discussed in Chapter Five, the discrepancy among the Drug Control Law, HIV Prevention Law and the Administrative Code Violation Law is seen as one of the main stumbling blocks that stops harm reduction from scaling-up. Therefore, for some interviewees, having separate laws for addiction treatment and harm reduction is necessary. These laws would allow key stakeholders to take clearer responsibilities regarding treatment, harm reduction and helping people who use drugs. Moreover, in order to make harm reduction more accessible (a lowthreshold approach), administrative hurdles should be removed. Nevertheless, reforming legal frameworks is not enough; some interviewees highlighted the importance of improving human resources for harm reduction.

\section{Human Resources}

In addition to reforming current relevant laws, some interviewees emphasised the importance of developing human resources not only for research, but for work at local levels. They suggested:

In other countries, they have research institutes on addiction, people who use drugs and drug policy ... for example, while Vietnam only has 10 people working at the governmental policy-making institution, in Cambodia, they have 600-700 people, Thailand has thousands ... Vietnam really lacks an intensive research agency (Police 1).

We need to improve human resources at local levels such as social workers, and consultants because the local or community based-treatment and support is essential (Doctor 6).

As emphasised previously, the misleading perceptions of drugs, drug use, addiction, and harm among study participants have led to skewed understandings and interpretations of the meaning of 'harm reduction'. Therefore, developing research and education on drug use and drug policy is important. Also, in order to support people who use drugs and promote authentic harm reduction, it is essential to have better human resources at local levels. As some interviewees highlighted above, this may be hard to realise in the near future given the limited finance allocated to harm reduction at local levels in Vietnam. But, as noted previously, Vietnam still prioritises abstinence and law enforcement activities in order to build a drug-free society, so convincing government to spend funding to recruit and train staff to 
help people who use drugs could take time. Yet a focus on helping people who use drugs is important, as this highlights the responsibility of the state for the vulnerability of individuals and their risk of drug-related harm (Hathaway, 2001). Removing harm is a human rights issue and it is a pillar of an authentic harm reduction philosophy.

\section{Acknowledging People who use drugs' Rights}

Some interview participants emphasised the importance of helping people who use drugs in a way that prioritises their rights:

We can learn some good examples from European countries like the Netherlands, it tries to help people who use drugs, and users are facilitated to speak up and to raise their voice ... they have freedom, they have the right to access legal, health and employment assistance ... they are protected by laws ... we also should have antistigma and discrimination polices for people who use drugs (Doctor 1).

We should find ways to allow people who use drugs easier access to treatment ... we should have more clinics ... have more overdose prevention medicines ... importantly the majority of people who use drugs want to have a job, so we should have policies that give users employment opportunities ... once they have a job they are less likely to use drugs again (Harm reduction official 5).

These suggestions reflect authentic harm reduction goals. They not only address medical issues, but, more importantly, promote people who use drugs' rights in ways that work against harmful abstinence-based policies. Acknowledging the benefits of people who use drugs' rights is an important step to developing harm reduction in Vietnam, as people who use drugs are often perceived as bad people who undermine moral traditions, have pathological failings, and threaten society. Considering people who use drugs' rights is necessary because, as Levy (2014) and the International Harm Reduction Association (2016) both argue, when people who use drugs are at risk from and suffer human rights abuses, harm reduction services can never properly function, and drug-related harms (like HIV infection and other blood-borne diseases) will be exacerbated. Similarly, researchers like Buchanan (2015b), Fedotov (2010), or Elliott et al. (2005) highlight that if people who use drugs' rights to health, education, or employment are jeopardised, this will further isolate users from 
normative society, and reduce their opportunities for education, outreach or work. All of this would exacerbate harm to people who use drugs, and others in the wider community. Notably, some research interviewees emphasised a long-term solution that focuses on addressing social and economic inequality.

\section{Addressing Social and Economic Disadvantages}

Many interviewees had a long-term vision for rational measures to address drug use and promote harm reduction. For example, one interviewee highlighted the importance of addressing poverty and developing education rather than focusing on demand and supply reduction:

The main problems here are not demand or supply reduction measures, we need to address poverty ... because social negativity is derived from poverty ... also we should raise awareness and improve education ... because we must accept that from historical perspectives it is impossible to have a drug-free society ... Vietnam should prioritise overcoming poverty as addressing drug use problems not supply or demand reduction (Harm reduction official 3).

Similarly, one government worker stressed the role of economic and social development as a long-term solution:

Having said that a drug-free society is impossible ... all over the world we have to live with drugs ... nothing is better than the economic and social development ... once the economy grows, education would be better and the standard of living would be also improved ... these are satisfactory answers for addressing drug use. People who use drugs want to have a decent job, they want to have a stable income ... if they have these things they can overcome any challenges (Government official 2).

Ideally, having a better standard of life can be an answer for many social problems, but, given Vietnam is a developing country, this may be hard. As noted previously, the problems that people who use drugs face emerge from social inequality, but also from prohibition. Overly punitive laws have arguably caused stigma and discrimination towards people who use drugs rather than providing help (GCDP, 2014; Trace, Somogyi, Hallam, \& Bewley-Taylor, 2007). 
In short, there were different suggestions on how Vietnam should move forward and make changes. Many stressed systematic reforms in law and policies, and collaborative work between government agencies. A few also recognised the importance of acknowledging the rights of people who use drugs and the need to address long-term social and economic problems. These progressive suggestions are significant because they are close to harm reduction goals that not only address medical issues, but more importantly tackle social, economic and cultural problems that people who use drugs face. Yet the general findings show that Vietnam still requires significant changes to fully develop and implement authentic harm reduction.

\section{Conclusion}

Overall, with the exception of some police and MOLISA professionals, the majority of research participants favoured harm reduction. Advocates believed that harm reduction is an effective measure to mitigate the harms of drug use and to promote the relationship between people who use drugs with community and services. However, it is evident that harm reduction is still perceived in narrow health terms - just a few respondents saw harm reduction in terms of evidence-based effective practice with people who use drugs, anti-discrimination or the promotion of human rights. Further, while some medical interventions like methadone, needle exchanges or naloxone received strong support, other harm reduction initiatives, such as providing injectable drugs or opening drug-consumption sites, faced stronger opposition. Those who disagreed with harm reduction argued that it fosters drug use, and they proposed that abstinence-based approaches (like supply and demand reduction measures) can reduce drug use and then reduce harm. Many study participants perceived that harms can only be limited through reductions in the demand and supply of drugs.

Alongside these misunderstandings, study participants highlighted many other financial, legal, administrative, and sociocultural barriers to harm reduction. Nonetheless, they recommended solutions to facilitate harm reduction. Emphasising the importance of closing down the 06 system and reforming current drug policy, they also suggested that the government should improve worker capacity, and develop cooperation between key stakeholders. Notably, some recognised the importance of acknowledging people who use drugs' rights and addressing long-term social and economic problems, the key pillar of 
authentic harm reduction. These ideas are essential to developing authentic harm reduction in the right way. However, to do this, Vietnam has a significant task to develop knowledge and change the contemporary understanding of harm reduction. 


\section{Chapter Ten: Discussion, Implications and Conclusion}

\section{Introduction}

This thesis has shown that Vietnamese policy-makers and government officials hold flawed perceptions of harm reduction philosophy. These perceptions have underpinned Vietnam's implementation of punitive policies and ineffective prohibition-based strategies towards people who use drugs. This chapter further explores how harm reduction is perceived and conducted in Vietnam, which is to answer the key research questions (noted in Chapter One). Drawing on previous studies, it argues that Vietnam has implemented 'pseudo' harm reduction, in which harm reduction is used to control people who use drugs. This so-called harm reduction is designed to protect wider society and to serve political purposes rather than minimise harm to people who use drugs. The chapter asserts that understanding 'authentic' harm reduction, and following its key principles, is vital to conduct meaningful harm reduction. It further highlights and consolidates the key principles of authentic harm reduction, in relation to: (i) how drug use is conceptualised, (ii) how it is responded to, and, (iii) how those who use drugs are treated. In doing so, the chapter raises some successful lessons of authentic harm reduction from other countries that Vietnam can follow and apply in practice. Finally, following scientific evidence, it develops suggestions on what the Vietnamese government should consider for future drug policy reforms. As a result, the study research questions were addressed in terms of what should be done to facilitate improvements in harm reduction strategies in Vietnam.

\section{'Pseudo' Harm Reduction in Vietnam}

In response to the drug use and HIV/AIDS epidemic during the 1990s, Vietnam introduced some medical initiatives (including peer education and outreach, methadone and needle exchange programmes) in the name of harm reduction. After nearly three decades, these initiatives have been developed nationwide. Over time, Vietnam's narrow version of harm reduction has often been used for political purposes (seen most clearly in the introduction of 06 Centres). Further, the shift in representing drug use and responding to it (from a social evil to a health matter and harm reduction) has been slow in practice. Thus, this thesis has an 
evident answer for the research question. That is, Vietnam has conducted pseudo harm reduction, which follows abstinence principles and is designed to protect wider society rather than people who use drugs. Vietnam's drug policy generally fails to adhere to authentic harm reduction principles.

Based on previous studies, drug use is still widely perceived as a serious social problem in society (Edington, 2016; Edington \& Bayer, 2013). As noted previously, drug use was regarded by Vietnamese authorities as a leftover of old regimes and a form of external contamination from the West and capitalism. Although the country has opened its economic policy to integrate global market liberalisation, state agencies still conduct nationalistic campaigns in a bid to counter the 'poisonous forces' of Western capitalism. Preventing drug use is seen as an important part of these campaigns. As a result of the HIV epidemic, and the ongoing prevalence of illicit drugs, drug use is also constructed as a social evil within Vietnam. Vietnamese leaders argue that drug use causes the degradation of young people, as it leads to addiction and crime (Edington, 2016). As a result, those who use drugs are detained in compulsory treatment centres in the name of rehabilitation and treatment. Those detained are used as free labour, and 'treatment' is diminished.

This study confirms the dominance of abstinence-based mind-sets among professionals in Vietnam. Except for interviewees working for public health and international sectors, the majority (Police, MOLISA workers, local authorities) thought that drug prevention is needed to protect the wider community and reduce harm. As noted in Chapter Four, under current conditions of the global capitalist economy, the Vietnamese state is less able to secure the economic wellbeing of its citizens. In turn, Vietnam has sought to secure its standing by emphasising that it can protect citizens from crime or other threats. This approach has contributed to the development of increasingly repressive discourses and practices to those seen as harming society, including people who use drugs. Vietnamese researchers, media, and government continually exaggerate the connection between drug use and addiction and crime, as well as the dangers of drug use. As a result, and as noted in Chapter Five, Vietnam has developed complex, often contradictory, approaches to drug use and people who use drugs, based on moral and brain disease models of addiction. Aiming to build a 'drug-free 
society', Vietnam's ensuing strategies have been ineffective and had limited impacts on drug demand and supply.

As a result, people who use drugs suffer from the controversial nature of Vietnamese drug policy, and they have been treated harshly. People who use drugs are widely perceived as having low levels of education, being unemployed and unemployable, causing anti-social problems, and going against conventional values. People who use drugs are also widely labelled as having deviant or criminal behaviours, so all people who use drugs are expected to be sent to treatment centres for crime prevention. These assumptions are not new and they are repeated worldwide. Yet as mentioned previously, those who use legal drugs like alcohol and tobacco cause more harms than illicit people who use drugs, but this fact is continually overlooked. Furthermore, much criminal behaviour related to drug use today is actually a direct result of prohibition; even under prohibition regimes, the vast majority of people with problematic drug use do not commit any crimes other than contravening drug laws (Global Commission on Drug Policy, 2017). Despite this, Vietnam still stereotypes all people who use drugs as problematic and potential criminals and it fails to recognise that most people use drugs recreationally.

The failure to acknowledge the complex nature of drug use and people who use drugs has resulted in misleading perceptions. In Vietnam, drugs, drug use and people who use drugs are generally regarded as the sources of harm caused by drug use. The findings consolidate this point. Most interviewees reflected on the 'addictive' and detrimental effects of drugs, and the criminal or anti-social behaviours of people who use drugs. As a result, Vietnamese professionals perceived that reducing harm means preventing illegal drugs and drug use, and controlling people who use drugs. They represented a form of pseudo harm reduction. These misleading perceptions are far removed from those found in authentic harm reduction philosophies. Authentic approaches do not seek to prevent drug use or control people who use drugs. Harm reductionists accept drug use as normal behaviour, they promote decriminalisation and help people who use drugs to make well-informed choices without any judgements. Meanwhile, in Vietnam understanding about harm reduction and implementing it in practice generally contradicts these principles. 
The following primary findings further illustrate and assert how the perceptions of Vietnamese key stakeholders are diametrically opposed to authentic harm reduction principles. Firstly, the general myth held by the study participants is that reducing harm means ceasing drug use, through punishment if necessary. This misunderstanding has resulted in a common thought that preventing drug supply and controlling demand equals harm reduction. As discussed above, supply and demand reduction is not authentic harm reduction because their aims differ from each other. Yet many interviewees, especially the police, believed that preventing drug supply is a form of harm reduction because (in their opinion) if supply is controlled, the harm associated with drug use can be mitigated. Another common myth is that sending people who use drugs to compulsory treatment centres is a form of harm reduction. These myths reconfirm the prevalence of abstinence-based perceptions among key stakeholders and demonstrate that the Vietnamese version of harm reduction is designed to reduce harm to society, not people who use drugs. These myths explain why the Vietnamese government continues to increase ineffective, counterproductive punishments and incarceration in the name of treatment and crime prevention.

Secondly, legal frameworks have also resulted in misleading harm reduction strategies. As explained previously, there are contradictions between the 2006 HIV Prevention Law, the Administrative Violation Law 2012 and the 2006 Drug Control Law. These contradictions make practitioners, especially police, confused about what harm reduction is. Most police (who follow the 2006 Drug Control Law) thought that preventing and combating drug use, and sending people who use drugs to 06 centres, are forms of harm reduction. This dilemma remains unchanged, even though Vietnam has legal frameworks for harm reduction (the 2006 HIV Prevention Law). Many interviewees believed that these inconsistencies in law and policy should not exist because they increase distrust among people who use drugs towards harm reduction services. Another contradictory issue relates to the 2012 Administrative Violation Law. On paper, Vietnam decriminalised illicit drug use in 2009 but, in reality, people who use drugs can be fined and detained in coercive centres if they use illicit drugs according to that law. Further, people who use drugs continue to be regarded as having moral and disease problems. They are widely judged and stigmatised, and suffer discrimination. These policies make it more difficult for people who use drugs to engage with harm reduction in practice. 
Thirdly, the findings reaffirm that judgemental attitudes, causing stigma and discrimination, are a long-term hurdle for authentic harm reduction in Vietnam. People who use drugs who participate in harm reduction services are still widely perceived as having pathological failings and being 'high risk'. This partly explains why people who use drugs' rights are politically ignored, as promoting their rights is believed to oppose the rights of the wider community. Instead, harm reduction is used as a mechanism to manage and control people who use drugs. For example, when people who use drugs seek to join harm reduction initiatives, they have to ask for a confirmation letter from local authorities first. This practice is far removed from an authentic harm reduction approach, which takes a low-threshold on access and places people who use drugs at the centre of services.

In general, the key research question has been answered. That is, Vietnam conducts pseudo harm reduction. Where this harm reduction is implemented, it is highly medicalised (from a narrow public health perspective) and designed to protect the wider community (e.g. from HIV) rather than assisting people who use drugs, or promoting their rights. The Vietnamese model of harm reduction is barely linked to the social support, such as employment or community re-integration. Also, the problems of Vietnamese prohibitions and drug policies seem to be overlooked. For example, Vietnam still receives financial funding from international donors to implement harm reduction, but it still supports the existence and even the expansion of the 06 centres. The centres, which are arguably the greatest barriers for authentic harm reduction, continue to be supported by Vietnamese politicians (mainly, it appears, as they are seen to offer work for centre leaders and staff). To assume that Vietnam's drug policy has transformed from punitive control measures to authentic harm reduction approaches is erroneous.

\section{'Authentic' Harm Reduction}

Globally, authentic harm reduction aims to reduce the adverse health, social and economic and other consequences of illegal drug use. It does not demand a reduction in drug consumption (Riley et al., 1999). Harm reduction is seen as an effective approach to address the complexity of drug use behaviours (the drug, set, and setting) and to meet the needs of people who use drugs (Ritter \& Cameron, 2006). As noted in Chapter Three, there are

different definitions around harm reduction philosophies, its implementation and its 
application by harm reductionists (Collins et al., 2012; Roe, 2005). Yet the key components of harm reduction include: (i) how to conceptualise drug use, (ii) how to respond to it, and (iii) how to treat people who use drugs.

Firstly, drug use is a reality of human nature, so it is irrational to legalise some drugs (like alcohol and tobacco) while prohibiting others (listed as illicit drugs) (Hofschulte, 2012; McNeece, 2003; Stevens et al., 2010). Whether governments accept drugs or not, drugs still exist and continue to be an important part of human lives (Gossop, 2012). Consuming drugs has historically been an almost universal phenomenon and the reasons for taking both legal and illegal drugs are generally similar. The use of drugs to relieve suffering, or for recreational and medical purposes is recorded throughout human history. Yet the Single Convention on Narcotic Drugs 1961, Psychotropic Substances 1971 and the Convention against the Illicit Traffic in Narcotic Drugs and Psychotropic Substances 1988 have changed how drugs are managed and controlled (Taylor et al., 2016). These UN Conventions list 'illegal' narcotic and psychotropic substances that require strict control due to their exacerbated harmful effects to human health and society, while other drugs, like alcohol and tobacco, which cause even more harm, are legally accepted within the UN agendas and across other countries, including Vietnam (Roberts et al., 2004).

From a harm reductionist perspective, the harms caused by illicit drug use are socially and politically constructed. As discussed previously, the UN, national politicians and the media mistakenly construct illicit drug use as an 'evil' that destroys families and young lives, and fuels a wide range of criminal activity (Buchanan, 2009). In turn, these perceived harms are used as reasons for prohibition and punishment. Yet as demonstrated previously, only a minority of illegal people who use drugs develop serious drug problems, like health or crime issues, and actually the vast majority of people who use drugs consume drugs recreationally. Therefore, no distinctions should be made between legal drugs, pharmaceuticals and illicit drugs because all drugs have both positive and negative attributes, and all drugs are potentially dangerous even though some are less harmful than others (Goldberg, 1999; Hunt et al., 2003). Drug use should, then, be viewed neutrally as drug use itself does not constitute a problem (Tammi \& Hurme, 2007). Holding a non-judgemental attitude towards drug use is a vital step to implement authentic harm reduction in practice. 
Secondly, from the standpoint of authentic harm reduction, drug use behaviour should not be prosecuted or punished (Harris \& McElrath, 2012). Within the harm reduction framework, acknowledging drug use as a high-risk behaviour is a product of a given time and culture; it is based on political factors rather than scientific evidence (Denning, 2001). As Hutton (2017) argues, abstentionists and governments have employed punitive drug policies due to a culture of fear towards people who use drugs who are seen as problematic. Although drug use can be harmful and people can be harmed by using drugs, most harm is caused by prohibitive and punitive drug policies (Buchanan, 2016a). In Vietnam, for instance, compulsory treatment centres (driven by punitive prohibition) isolate users from society, leading to the reduction in their opportunities for education, outreach, and employment, as well as increasing their stigma and discrimination (Global Commission on Drug Policy, 2014). Acknowledging these counterproductive effects of prohibition, harm reductionists promote the decriminalisation of drug use and essentially state that people who use drugs should not be punished or detained in the name of treatment or harm prevention. Anti-discrimination is an essential component of authentic harm reduction.

Furthermore, authentic harm reduction provides people who use drugs with pragmatic measures that help them to reduce the harm caused by drug use. This means that service providers should seek the best possible approaches to suit the needs of people who use drugs, whether they want to continue drug use or want abstinence. Authentic harm reduction is practical and pragmatic. It is a client-centred approach that maximises positive outcomes and minimises negative consequences. Pragmatism means a requirement for evidence-based practices, not dogmatic and coercive actions. Vietnam has not yet used scientific and empirical knowledge as the foundation for implementing harm reduction. Instead, as stated previously, it uses pseudo harm reduction to serve its political and economic incentives. Therefore, the rights of people who use drugs, a vital component of authentic harm reduction, have generally been overlooked in Vietnam.

Thirdly, authentic harm reduction is a humanistic and non-judgemental approach that respects the dignity and self-determination of people who use drugs and how they want to address their drug use problems (Riley \& O'Hare, 2000). Drug consumption is seen as a personal choice, so people who use drugs should be given the opportunities to make well- 
informed choices about how they want to resolve their problems (Goldberg, 1999, 2004). For instance, people who use drugs will decide how best to address addiction, alongside the support of service providers (Fernandez, 2012). In situations where people who use drugs become addicted and continue to use drugs and relapse, this should be seen as a natural, expected occurrence and a learning experience (MacMaster, 2004; Marlatt, 2000). Allowing people who use drugs the rights of self-determination will generally facilitate the treatment process. This is rarely the case in practice. When I joined an international conference on drug policy in Denmark, ${ }^{78}$ most researchers and practitioners highlighted the role of respecting the rights of people who use drugs when it comes to implementing authentic harm reduction. They emphasised that harm reduction is never successful when human rights are not centralised. Unfortunately, Vietnam has not yet taken human rights issues into serious consideration when implementing interventions in the name of harm reduction. This indifference has resulted in pseudo harm reduction.

Furthermore, authentic harm reduction is heavily based on a low threshold approach in which minimal requirements are imposed upon people who use drugs if they want to participate in harm reduction interventions (Blalock-Wiker, 2014; Denning \& Little, 2011; Hilton et al., 2001). This approach is essential. As noted previously, in Vietnam, most people who use drugs do not seek treatment because of stigma and discrimination and the risk of long-term detention in compulsory treatment centres (Vuong et al., 2016). From an authentic approach, the role of treatment centres and service providers should be to create a friendly and nonjudgemental environment in which people who use drugs can discuss and make decisions about their treatment (Fernandez, 2012). Given the diversity of reasons behind how or why people take drugs, and become addicted to drugs, authentic harm reduction interventions should ideally be tailored on an individual level. Placing people who use drugs at the centre of supportive services is indispensable to implement authentic harm reduction. I participated in a conference on harm reduction in Canada, ${ }^{79}$ and most researchers and practitioners stated

\footnotetext{
78 I attended the $11^{\text {th }}$ conference of the International Society for the Study of Drug Policy, held at the Centre for Alcohol and Drug Research, Aarhus University, Denmark. The conference took place between 17 and 19 May 2017.

${ }^{79} \mathrm{I}$ joined and presented at the 2018 Conference on Harm and Harm Reduction in the Criminal Justice System, the $8^{\text {th }}$ National Conference on Critical Perspectives: Criminology and Social
} 
the importance of taking non-judgemental and respectful attitudes, and to make assessments that consider the context of a client's life. Further, harm reduction practitioners need to understand the reasons for a client's behaviour or decision, to accept the client's decision, and to give advice and recommendations based on that evidence and context. By doing these things, authentic harm reduction can reach people who use drugs and deliver care whenever needed.

In short, influencing drug policy and responses in a way that is more humane and meets people who use drugs' needs (social and psychological supports) is essential for authentic harm reduction. If authorities fail to ensure the human rights of people who use drugs or to offer necessary assistance, people who use drugs will more likely experience stigmatisation and discrimination, which causes harms at individual and community levels (Ezard, 2001; Hathaway, 2001). Reforming punitive and abstinence-based drug policies towards authentic harm reduction means opening people who use drugs' access to their legitimate human rights, including rights to health, education, or employment. For Vietnam, this requires a significant reformation of drug and social policies.

\section{Implications of the Study}

In Vietnam, pseudo harm reduction is prevalent. Where harm reduction measures are in operation, they are implemented from abstinence frameworks, not authentic harm reduction principles. As demonstrated previously, despite having some critiques (discussed in Chapter Three), authentic harm reduction has been generally proven as an effective method to address drug use worldwide. Therefore, it should be expanded, promoted, and conducted properly in Vietnam. However, given the Vietnamese context (in which drug use is historically and politically regarded as a social evil), it is important to find ways to implement authentic harm reduction initiatives that suit the country's culture and politics. Many Vietnamese key stakeholders hold misperceptions of drugs, drug use, people who use drugs, and policy responses that are contradictory to authentic harm reduction principles. Therefore, many changes should be made to pave the way for authentic harm reduction in Vietnam.

Justice. This conference was held on 21 and 22 June at Stain's Marry University, Halifax, Nova Scotia, Canada. 
This section starts with some successful lessons from international contexts that could be applicable in Vietnam. The case study of Portugal demonstrates that decriminalisation has been working in practice as it has contributed to reducing problematic drug use and the stigmatisation of and discrimination against people who use drugs, which is widespread in Vietnam. The second lesson comes from the reclassification of cannabis in the UK. This reclassification has reduced the prevalence of drug use in the UK. Cannabis was classified as one of the most dangerous drugs in Vietnam, despite no recorded deaths from cannabis. Therefore, reducing sanctions for illicit drug use like cannabis is necessary to reduce harm caused by prohibition. The third lesson in Switzerland is related to prescribing pharmaceutical grade heroin to heavily addicted heroin users. This lesson is important because heroin is the most popular drug in Vietnam and many Vietnamese people who use drugs are addicted to this drug. The Swiss model may take time to adopt in Vietnam, but it is worth considering, given the prevalence of heroin-related problems in the country. If Vietnam can apply this model, this will be a great move to authentic harm reduction. These international lessons may give Vietnam confidence to expand harm reduction initiatives. From here, the recommendations to enhance authentic harm reduction in Vietnam include: (i) complete decriminalisation of drug use, (ii) gradually closing the system of compulsory treatment centres, (iii) removing legal framework barriers, (iv) enhancing the Vietnamese perception of authentic harm reduction, (v) improving human resources, and, (vi) ensuring people who use drugs' legitimate rights.

\section{Lessons from Drug Reform Changes}

\section{Decriminalisation in Portugal}

Following a difficult time with high levels of problematic drug use, Portugal became the first European country to decriminalise all personal possession of drugs in 2001. Notably, after removing criminalisation and punishment, the country has not seen any significant differences in levels of illicit drug use compared to previous times and other European countries. This fact reaffirms that prohibition has limited impacts on drug use. Since decriminalisation, there has been a remarkable decrease in the stigmatisation of and discrimination against people who use drugs (Global Commission on Drug Policy, 2017). As a result, problematic drug use has reduced and the numbers of people who use drugs joining 
in harm reduction and drug treatment services have increased. This outcome has resulted in considerable decreases in rates of mortality due to overdose, and new incidences of bloodborne diseases (Hughes \& Stevens, 2010). In addition, the number of new people who use drugs who are diagnosed with HIV has declined by $17 \%$ and there has been an overall drop of 90\% in drug-related HIV infection since the implementation of decriminalisation (Hughes \& Stevens, 2010). Also, the drug policy's reform has increased the health response to drugs and treatment approaches for problematic/addicted users in the country (Greenwald, 2009). Portugal's case demonstrates that decriminalising possession and use of all illicit drugs does not lead to an increase in drug use or drug-related harm. Clearly, less punitive responses result in more effective outcomes.

\section{Re-categorising Illicit Drug Schedules in the United Kingdom}

Based on reports of the Advisory Council on the Misuse of Drugs (2002), the UK government rescheduled cannabis from class $B$ to class $C$ in 2004 . There were considerable media debates between 2002 to 2004 regarding whether or not decriminalising cannabis would lead to an increase in drug use among young people, or bring addiction and mental health problems (Buchanan, 2015b). Yet under political pressure rather than scientific evidence, the UK government reclassified cannabis from class C to class B in 2008 (Nutt et al., 2010). The most noticeable fact is that the level of cannabis use decreased when cannabis was class C (20042008). The British Crime Survey revealed that from 1996 to 2002 the use of cannabis remained stable at 17\%, while between 2003 and 2008 the figures for cannabis use dropped from $14.1 \%$ to only 9.7 \% (Buchanan, 2015b). Notably, when cannabis was categorised into Class B again, cannabis use increased, ranging between 11-15\% between 2008 and 2015 (European Monitoring Centre for Drugs and Drug Addiction, 2017). This evidence indicates that lowering the sanctions for drug possession generally did not lead to increases in drug consumption in the UK. In general, prohibition has no effect on levels of drug use. Therefore, as discussed previously, reducing and moving away punishments for people who use drugs helps them to mitigate harm caused by prohibition, and increase their opportunities to stabilise their lives and access harm reduction services. 
While there may be a willingness to accommodate and legalise cannabis, some people consider that illicit drugs like heroin are extremely dangerous and result in heavy addiction and death if consumed frequently or heavily. Nevertheless, heroin-assisted treatment programmes in Switzerland have demonstrated that this is not necessarily the case (Stevens et al., 2010). In response to the heroin crisis in the 1980s, the Swiss government shifted its approach to drug use from a punitive-based approach to a public health-based one (Csete, 2010). Although speculative public concern that the programme would lead to serious problems, such as increased drug use, prolonged addiction and drug uptakes among young people, the programmes have succeeded immensely (Uchtenhagen, 2010). For instance, between 1991 and 2009, deaths caused by heroin overdose were reduced by 50\% and heroin use decreased by around $80 \%$ (Csete $\&$ Grob, 2012). Importantly, the Swiss model of harm reduction contributed to significantly improving the health and wellbeing of people who use drugs and connecting them with health and social services. As a result, criminal activities like acquisitive crime showed a major reduction (Strang, Groshkova, \& Metrebian, 2012). The Swiss model showed that authentic harm reduction contributed to positive and social outcomes without causing harm to people who use drugs and wider society.

These case studies of authentic harm reduction from Portugal, the UK, Switzerland and also from other countries discussed previously (drug consumption rooms in Australia and Canada; cannabis decriminalisation in the Netherlands) illustrate the ineffectiveness of prohibition and the positive outcomes of authentic harm reduction approaches. Importantly, these successful examples reaffirm that the wider shift from a criminal justice approach to a health and human rights oriented model of harm reduction is feasible and workable in practice. These examples give confidence for countries, including Vietnam, to step away from punitive responses to illicit drug use and to reform drug policy in authentic harm reduction ways.

\section{Policy Recommendations for Vietnam}

The international strategies to implement authentic harm reduction demonstrate how remarkable and immediate positive changes can be implemented. Such international harm reduction models cannot be entirely replicated in Vietnam, as Vietnam is socially, politically, and economically different from those Western countries. However, it remains possible to 
introduce effective reforms to develop harm reduction. These reforms include: (i) decriminalising drug use, (ii) gradually closing the system of compulsory treatment centres, (iii) removing legal framework barriers for implementing authentic harm reduction, (iv) enhancing the Vietnamese perception of authentic harm reduction, (v) improving human resources, and, (vi) ensuring people who use drugs' legitimate rights.

\section{Decriminalisation of Drug Use}

If Vietnam accepts drug use is a normal behaviour, and commits to treating people who use drugs as patients not criminals, then it should not punish users. Therefore, drug possession for personal use needs to be completely decriminalised. This means that the 2012 Administrative Violation Law would require amendment to remove sanctions for those who use illicit drugs. In addition, the 2015 Criminal Law should remove Article 249, which regulates that those who have already been sanctioned for administrative violations would be imprisoned for between one and five years. It is apparent that the 2012 Administrative Violation Law and the 2015 Criminal Law still put PWUD at risk of criminalisation and imprisonment. If Vietnam has committed to decriminalising drug use with the United Nations, they should fulfil their commitments and apply other alternative approaches, like 'authentic' harm reduction, for responding to drug related harms.

The decriminalisation of drug use would protect people who use drugs (particularly the poor, homeless and street people) who are targeted by police crackdowns, drug testing and drugrelated arrests, which lead them to coercive detention and incarceration. People who use drugs would avoid a drug conviction that can ruin their lives, such as stopping them from overseas travel, working or finding employment. The lessons from decriminalisation approaches in Portugal, Netherlands or Canada are useful to reflect upon, as they demonstrate that decriminalising the possession and use of illicit drugs has not led to increases in drug use or drug-related harm. Decriminalisation would help the Vietnamese government to reduce the wastefulness of the police pursuit of minor offenders and would allow the government to allocate more funding for health and social services to address drugrelated harm or problematic drug use as needed. Evidently, less punitive responses lead to more positive outcomes. 


\section{Scaling Down Compulsory Treatment Centres}

Compulsory treatment centres have been heavily criticised as ineffective and counterproductive. Once labelled as a centre detainee, a drug user encounters high levels of stigma and discrimination; this is a significant barrier for them to engage with harm reduction services. Retaining the centres is costly, while the treatment is ineffective, and even increases the rate of HIV transmission among people who use drugs. Scaling down the number of centres is necessary, not only for gradually removing barriers for effective harm reduction, but to free up the funding allocated for it. International workers and harm reduction officials estimate that if Vietnam closes down about half of the centres, it will have enough money to develop authentic harm reduction services. Yet, it must be acknowledged that these centres are entrenched in political and policing mind-sets. Therefore, expecting an immediate closure is almost impossible, but scaling down gradually and changing the functions of the centres are feasible.

As demonstrated previously, harm reduction programmes have resulted in a decrease in drug related crimes and an increase in the process of reintegration of PWUD into society. They should be closely integrated into all of the 06 centres. For instance, in the first phase, the centres should develop harm reduction, like needle and syringe programmes, to reduce the transmission of HIV among PWUD, and they should promote the wider distribution of methadone and buprenorphine. Next, the centres should provide other physical and mental health assistance and social services in the way that alleviates adverse health, social and economic consequences caused by drug use without requiring abstinence. The 06 centres also need to have more nurses, doctors, peer support workers, counsellors, and mental health workers. In general, if Vietnam cannot be closed entirely the centres, it needs to change the centres' focus and philosophy from punishment to authentic harm reduction.

Furthermore, Vietnam should close centres that are too far from communities and less accessible to PWUD, and importantly promote more community-based voluntary addiction treatments, like methadone. The study conducted by Vuong et al. (2016), which compares the effectiveness and cost-effectiveness of compulsory treatment centres and communitybased voluntary methadone treatment in Hai Phong City, Vietnam from 2012 to 2014, is worth considering. This study shows that voluntary methadone treatment outperformed 
compulsory treatment, as it was both less expensive and more effective. As a result, more voluntary methadone treatment programmes have been implemented in the country. In fact, most studies related to 06 centres highly recommend the reduction and closure of these centres, to pave the way for authentic harm reduction located within communities.

\section{Removing Legal Framework Barriers for Implementing 'Authentic' Harm Reduction}

Vietnam's current legal frameworks for harm reduction are unclear and even contradictory. Authentic harm reduction is a low threshold approach based on non-judgemental attitudes, so any legal foundations need to facilitate these approaches within service provisions. For example, administrative barriers need to be removed to make harm reduction more accessible to people who use drugs. Asking people who use drugs for too many administrative requirements to join harm reduction (such as personal application, an introduction letter from family and a local authority) is contradictory to a low threshold approach. These procedures discourage people who use drugs from joining harm reduction services. From a harm reductionist perspective, people who use drugs should not be judged when participating in harm reduction and should have easy pathways into services.

As noted in Chapter Nine, study participants recommended that Vietnam should separate out laws for addiction treatment and harm reduction. These laws would allow key stakeholders to take clearer responsibilities regarding treatment, harm reduction and helping people who use drugs. A harm reduction law should make clear that those who take drugs can take part in harm reduction services no matter who they are, where they come from, or which types of drugs they use. These legal foundations will allow practitioners to connect PWUD with services. Further, PWUD should not be monitored or arrested by police once they join harm reduction services. In fact, harm reduction should be provided for any drug users who identify a need for the service, not just for those meeting administrative requirements.

\section{Enhancing the Vietnamese Perceptions of 'Authentic' Harm Reduction}

The limited and mythical understandings about authentic harm reduction are major challenges for its implementation in practice. Changing perceptions takes time and needs education and training on many levels. Given Vietnam's context in which the centralisation of power is dominant, harm reduction related education and training should start with key 
stakeholders and top leaders. Developing their professional understandings will have a strong impact on how harm reduction is implemented in practice. Next, local leaders and authorities should be provided with sound knowledge pertaining to drugs, drug use, and authentic harm reduction. After this training, it should be expanded to those who work at local levels. Harm reduction training courses, based on scientific evidence, should be also integrated into police and MOLISA schools. Further, practitioners and the media should spread evidence-based knowledge of authentic harm reduction to the general public. By doing so, harm reduction may be properly understood across multiple professional and social sectors.

\section{Improving Human Resources}

Many study interviewees emphasised the importance of developing human resources for harm reduction interventions. Expanding human resources for harm reduction is difficult at the moment because of chronic underfunding. Nonetheless, redistributing current human resources is easier to carry out. For example, those who work for MOLISA and law enforcement agencies can and should be trained to be important resources for harm reduction and, once trained (in police and MOLISA schools), can add to the numbers of current harm reduction practitioners. Also, if systematic and lengthy training is provided (in all police, MOLISA and $\mathrm{MOH}$ schools), they will become active and long-serving proponents of harm reduction. By doing so, the Vietnamese government does not have to spend more money on recruiting harm reduction practitioners. Furthermore, as a police trainer, I will propose and carry out some new ways of police training. For example, my students can be trained and provided with sound knowledge and scientific evidence that will help them to have nuanced views on, and comprehensive understandings about drug use, PWUD, drug policy and harm reduction - not only in Vietnam, but in the wider global context. They (my students) may then become important resources of harm reduction practitioners in the future.

Ensuring People who use drugs' Legitimate Rights.

People who use drugs' rights are an essential element of authentic harm reduction. Yet human rights issues in general and the rights of people who use drugs, in particular, are a politically sensitive matter in Vietnam, so using the term 'human rights' may encounter non- 
cooperation and opposition from the Vietnamese government and local authorities. Given my role working as a police trainer, I think the best way to convince Vietnamese authorities is to avoid the term 'human rights', instead replacing it with another alternative like 'People who use drugs' legitimate interests' ${ }^{\prime}{ }^{80}$ It is important to persuade the government that considering people who use drugs' interests is necessary because, when people who use drugs are at risk from and face social and political disadvantages, harm reduction services can never properly function, and drug-related harms (like HIV infection and other blood-borne diseases) are exacerbated. In other words, if people who use drugs' legitimate interests to health, education or employment are jeopardised, this will exacerbate harm to them and others in wider society. Promoting human rights, the code of harm reduction, is going to take time, but it is possible if the current perceptions of drug use, people who use drugs, and harm reduction are changed.

Also, it is vital to help Vietnamese leaders to realise that helping users and ensuring their legitimate rights is going to help society in the long-term. Vietnam wants to protect the wider community through the existing version of harm reduction, but policy-makers and government fail to recognise that social safety is strongly linked with ensuring people who use drugs' interests. Authentic harm reduction aims to help people who use drugs to stabilise their lives by supporting their rights to health, education, accommodation, employment, and other social supports. Successful examples from the Netherlands or Canada (where people who use drugs are provided with health care, housing, employment, addiction treatment, and other supportive services) are good models for Vietnam to follow. Some study interviewees suggested that Vietnam should address the social and economic disadvantages associated with people who use drugs as a long-term solution, rather than focusing on demand and supply reduction. Their progressive thinking is significant for the development of authentic harm reduction in Vietnam. The country should change and expand the goals of harm reduction in order to address wider social harms, not just the medical harms associated with drug use. In fact, authentic harm reduction is possible and feasible for Vietnam if key

\footnotetext{
${ }^{80}$ From a Vietnamese government perspective, human rights are widely used by the USA and other external forces as means to force Vietnam to change their political system. People who use drugs' rights are part of political pressure that Vietnam has to cope with, so it is very difficult to convince and work with Vietnamese authorities if human rights issues are mentioned.
} 
stakeholders understand that authentic harm reduction benefits both people who use drugs and the wider society in the long run.

\section{Thesis Conclusion}

Since the beginning of the $20^{\text {th }}$ century, global responses to drug use have tended to engage with abstinence-based perspectives. Abstinence is preferred by most governments and policy-makers because it is thought to reduce drug consumption and crimes, and mitigate the harms caused by drugs. In turn, punishments and coercive drug treatments are prioritised as methods to minimise the harms caused by drugs and addiction. Yet there is little scientific evidence to demonstrate that forced abstinence-based approaches have yielded positive outcomes (Global Commission on Drug Policy, 2014). The evidence indicates that such forced approaches cause more harm and offer limited benefits to either people who use drugs and others (Taylor et al., 2016). The current responses to drugs are driven by myths, flawed assumptions and political populism, not necessarily evidence, science and rationality (Hutton, 2017; Taylor et al., 2016). Therefore, abstinence approaches fail to tackle fundamental drug use problems.

Authentic harm reduction, an approach that emerged from the 1980s when the HIV/AIDS epidemic became a serious problem, is seen as an effective approach to address the complexity of drug use behaviours and meet the needs of people who use drugs (Ritter \& Cameron, 2006). Harm reduction has shifted the way people and governments react to drug addiction and drug use problems (Harris, 2012). With many benefits, such as reducing HIV infection rates, promoting human rights, reducing stigma or crimes, authentic harm reduction has been seen as an alternative paradigm of dealing with both problematic and recreational drug use.

Authentic harm reduction engages with the diversity of human experiences and behaviours in relation to drug use. The key principles of authentic harm reduction reflect how and why drug use should be perceived and how people who use drugs should be treated in order to address not only their problems but also, more broadly, community and societal problems associated with drug use. Harm reductionists try to change attitudes and behaviours among users, the public, government workers, and international organisations to develop more 
advanced laws, policies, and practices to live with drugs (legal and illegal) and to minimise their harm. Throughout its history, harm reduction has mainly focused on public health concerns (addressing HIV infection, other blood-borne diseases and addiction), but this is no longer the sole focus (Harris \& McElrath, 2012). Over the course of the last decade, authentic harm reduction has expanded its scope to address the multiplicity of harms associated with drug use at the individual, community, and societal level (Marlatt \& Witkiewitz, 2010). In fact, many harm reductionists have increasingly used 'human rights' as the discursive framework for harm reduction (Stimson, 2007). Some harm reductionists even go further than public health and human rights, and they promote drug law and policy reform that emphasise decriminalisation and aim to reduce all harms caused by prohibitionist drug policy.

While Vietnam may outwardly express a commitment to harm reduction, it does so within a broader philosophy that expects lifelong abstinence. This derives from the history of drug use in Vietnam, which is complex and multifaceted. Throughout Vietnamese history, drug use has been negatively viewed. It was first seen as a national enemy of the state and it was then cast as a poisonous force and a social evil in Vietnam. The increasing numbers of people who use drugs and the development of new popular drugs and the increasing HIV infection rates among people who use drugs have further consolidated negative attitudes towards drug use in Vietnam. Accordingly, the Vietnamese government has tended to produce narrow and misleading representations of the harms associated with drug use, and exaggerated these harms. These perceptions have overlooked the complexity of drug use (including the dominance of recreational use) and the role of wider social, environmental and legal factors in creating harms. Vietnamese perceptions of drug use are influenced by political and sociocultural factors rather than evidence (Edington, 2016). Stigmatising and discriminatory representations of drug use and people who use drugs have been developed by the media and Vietnamese leaders. Further, the effects of market liberalisation on national identity have tightened Vietnam's controls of drug use (Wilcox, 2000), placing it as an immoral act that represents negative Western values (Rydstrøm, 2006).

Vietnam's drug policy is now considered to be among the most punitive in the world. While engaging in some medical interventions in the name of harm reduction, current responses to drug use remain deeply punitive and abstinence-focused (Vuong, 2016). Vietnam has focused 
on deterrence by prioritising abstinence-based approaches that include the suppression of drug supply and demand. A wide range of reduction measures have been implemented, but their effectiveness is limited and even counterproductive, resulting in negative consequences, including human rights violations and ineffective, costly treatments. Since the 2000s, Vietnam has implemented some so-called harm reduction measures but little attention is paid to address the social, psychological, and environmental disadvantages that hamper people who use drugs' treatment and community re-integration. Current repressive law enforcement, including the compulsory detention of people who use drugs, has combined with a range of barriers (such as insufficient funding, social stigma, and political factors) to authentic harm reduction. Vietnam's current version of harm reduction is designed to protect the wider community from HIV rather than assisting people who use drugs, or promoting their rights and benefits.

This study has confirmed that Vietnam has embraced a pseudo form of harm reduction. That is, only a few informants believed that people can take drugs recreationally, and the majority thought that all illicit people who use drugs have to struggle with addiction. The most common myth among Vietnamese people is that drug use causes crime. This perception has resulted in the prevalence of 06 centres as a crime prevention measure. These common myths have strong impacts on how Vietnam approaches the harms caused by drug use. For example, no interviewee mentioned the harms caused by current drug policy. In fact, most respondents still believed that reducing harm means eradicating the supply and increasing punishments for those possessing, trading, and smuggling illicit drugs or producing precursors. They also tended to think that controlling and punishing people who use drugs is necessary to reduce harm, and the best way is to send people who use drugs to forced treatment. Police and MOLISA professionals frequently argued that the centres contribute to reducing the crime rate and protecting the wider community and, as such, were a form of harm reduction.

Nevertheless, there were a number of criticisms associated with compulsory treatment centres. Many interviewees, particularly public health and international workers blamed the centres for being ineffective and costly, with high relapse rates, poor clinical care, and minimal treatment services. The vocational training on offer is deemed irrelevant to the labour market. Some international interviewees saw that the centres are maintained for the 
economic wellbeing of centre staff and leaders. These are new findings. Such findings cement the fact that responses to drug use, addiction, and people who use drugs tend to ignore the complexities of drug use behaviours and the intersections between drug use and political, social, or environmental factors. Also, they confirm that Vietnamese responses are not based on evidence and rationale. They are, instead, a form of pseudo harm reduction.

This study also shows that, with the exception of some police and MOLISA professionals, the majority of research participants favoured current versions of harm reduction. Those who disagree with harm reduction argued that it fosters drug use, and that abstinence-based approaches can reduce drug use and thereby reduce harm. Study participants frequently perceived that harms can only be limited through reductions in the demand and supply of drugs. Meanwhile, advocates believed that harm reduction is an effective measure to mitigate the harms of drug use and to promote better relationships between people who use drugs, community, and services. However, they retain perceptions based in narrow health terms. Few see harm reduction in terms of evidence-based effective practice with people who use drugs, anti-discrimination, or promoting human rights. Further, while some medical interventions like methadone, needle exchange or naloxone received strong support, other harm reduction initiatives, such as providing clean drugs or opening drug consumption sites, faced strong opposition.

These perspectives have hampered authentic forms of harm reduction from taking place. Harm reduction has also been undermined by other barriers such as financial, legal, administrative, and sociocultural obstacles, including stigma towards people who use drugs. Study participants were often well aware of these barriers and they suggested numerous changes, which addresses the study research questions in terms of how problematic perceptions impact on harm reduction and what should be done to facilitate improvements in harm reduction strategies in Vietnam. They noted that the 06 system needs to be scaled down and then closed, and the current drug policy should be reformed. Also, they recommended that the Vietnamese government should improve human resources and cooperation between key stakeholders. Some recognised the importance of acknowledging people who use drugs' rights and addressing long-term social and economic problems related to people who use drugs. These findings show that Vietnam still needs significant change, 
especially in understanding the nature and potential of authentic harm reduction, to develop it properly. Without changing current approaches, Vietnam continues to conduct pseudo harm reduction, which causes harm instead of reducing it.

\section{The Strengths of the Thesis}

There has been a lack of critical and systematic exploration of how professionals perceive drugs, drug use, those taking drugs and drug policy in relation to harm reduction strategies in Vietnam. For this fundamental reason, this thesis significantly adds value to the theoretical and practical understanding of harm reduction both in Vietnam and also in the wider global context (especially South East Asia). In addition, harm reduction has developed in western countries, so country specific research of this kind in a non-Western context is extremely valuable to see whether or not harm reduction can be applicable for different countries with different political systems.

This thesis is the first piece that explores how professionals understand and implement drug policy in conjunction with harm reduction philosophies from a Vietnam perspective. The results of the study expose both the lip service paid to harm reduction by the Government while pursuing an abstinence-based approach. Accordingly, the thesis concludes that Vietnam has embraced pseudo harm reduction. The study also contributes a resource of knowledge to those who are seeking to inform and shape drug policy in Vietnam. Importantly, it provides the foundations for the necessary changes for implementing authentic harm reduction in Vietnam.

\section{Research Limitations and Future Research}

While this study has made important contributions to the literature, some limitations were identified. As noted in Chapter Six, there was a form of bias, as there were more respondents working for law enforcement and social work sectors compared with those working in other sectors who participated in the survey and interviews. This over-representation reflects the dominant role of law enforcement and social workers in addressing drug use related problems within Vietnam. Importantly, as shown by the study findings, police and MOLISA officials generally opposed and misperceived harm reduction strategies, so future researchers may try to focus on these groups and help them to engage in authentic harm reduction more actively. By doing so, scaling up authentic harm reduction in Vietnam may be more achievable. 
Another limitation is that this thesis has not yet deeply unpacked the survey results, as the study heavily relied on the qualitative data. The study has a lack of engagement in multivariate analysis with further statistical significance testing. Some of the other demographic information (e.g. age, gender, setting, qualification, or years' experience in the field) might have influenced responses. The data is ordinal, so consideration might be given to factor analyse the questions, group them, and then engage in relevant multivariate analysis (e.g. ANOVAs, regression analysis). Although the key focus of the study is qualitative data, further exploring the multivariate statistical analysis of survey questions will be interesting and valuable. Therefore, future peer reviewed publications should consider quantitative data analyses.

Finally, this thesis is limited in scope as it only explores the perspectives of people who work in the drug field and harm reduction, and not drug users or their families. As noted previously, from the standpoint of harm reduction, drug users and their families play a key role in addressing their problems. Other research could be extended from this study by collecting data from drug users and their family members to produce richer and more diverse results. In other words, even one study about how Vietnamese people who use drugs and their families perceive harm reduction would significantly add to new knowledge about authentic harm reduction.

\section{Personal Reflection}

This has been an amazing four years of my life. I have experienced a huge shift in my understanding about social sciences, particularly my perceptions of harm reduction, during my PhD study. At first, I found it difficult to adapt to a new culture and new people in New Zealand. Honestly, I struggled to understand the Kiwi accent during my initial six months. In addition, I encountered challenges in intensive English reading and writing, which is not my first language. Over time, I have improved my English and importantly my understanding about academic knowledge, work and the concept of harm reduction. Given my role as a police officer in Vietnam, I usually followed the party-line approach, so I did not really have critical perspectives in looking at social issues. In other words, most of my knowledge was generally regulated and based on the instructions of the MPS. The research process has remarkably changed my perceptions of the social world and allowed me to assess a reality 
(harm reduction in Vietnam) that exists apart from my own perceptions. This research has allowed me to separate my previous thinking and to develop better and wider understandings about how people perceive this reality.

The interpretivist approach, in particular, has allowed me to see that social reality is differently experienced and perceived by different people. As noted previously, asking public health and international workers about 06 centres was straightforward, while those working for MOLISA were more difficult as they were so cautious. I now understand that different people view and understand the same 'reality' from different angles. This is a great change in my thinking. When I was in Vietnam and working for MPS, I frequently followed and believed in what was written in Vietnamese government documents or books for police. Therefore, a narrow and even short-sighted view was inevitable. It means that if these documents say that drug use is extremely harmful and has no positive implications, I would not challenge or argue these assumptions. Nevertheless, during my PhD journey, I have realised that Vietnam's government has exacerbated the risks and dangers of illicit drugs and people who use drugs, which are based on political factors rather than evidence. By interviewing professionals and also working with my supervisors, it becomes clear to me that what I know is only the tip of the iceberg. The drug use phenomenon is a complex and multifaceted issue that needs to be considered from different perspectives. Drug use can be harmful, but most harms are caused by punitive Vietnamese drug policies. I did not realise this fact when I was working as a police officer, tasked with preventing drug use at any cost. Thanks to doing this PhD and working with different professionals, researchers, and my supervisors, I have become much more objective and developed my critical thinking. Yet taking this stance, and developing this evidence related to drug use, people who use drugs, abstinence and harm reduction, may make things challenging when I go back to work in Vietnam.

I can imagine that when I return to Vietnam to continue my job as a police and police trainer, it will be challenging if I say that drug use can be harmful, but most harms are caused by forced abstinence and drug policy. Julian, my former supervisor, once said that 'you are going to be in a very lonely position when you work in Vietnam because your progressive thinking is so different from the general mind-sets of your leaders and citizens'. Lizzy and Fiona, my current supervisors, have also realised this concern when it comes to my job security when I return to work in Vietnam. In fact, applying what I have learnt from my PhD is going to take 
time. Further, I expect that many people will think my study arguments go against the partyline and are influenced by western countries and culture. For example, considering the rights of people who use drugs, who are widely perceived as a high-risk group and a burden to society, is a folly. Nevertheless, Fiona, my supervisor, had a great idea that will help my research to be more easily accepted in Vietnam. That is, abstinence is an option for all people who use drugs and the role of harm reductionists is to help users to reach it by collective efforts and mutual discussions; yet enforced or imposed abstinence is problematic. I think this is a valuable argument that many Vietnamese people and leaders will agree with. Of course, 'easier said than done', but I have faith that my study could be accepted because it is based on scientific evidence and the perceptions of study participants, not my personal opinions. 


\section{References}

Advisory Council on the Misuse of Drugs. (2002). The classification of cannabis under the Misuse of Drugs Act 1971. Retrieved from Home Office, London, UK: https://assets.publishing.service.gov.uk/government/uploads/system/uploads/attac hment data/file/119126/cannabis-class-misuse-drugs-act.pdf

Ahmed, T., Long, N. T., Huong, P. T. T., \& Stewart, D. E. (2014). HIV and injecting drug users in Vietnam: An overview of policies and responses. World Medical \& Health Policy, 6(4), 395-418.

Akers, R. L. (1991). Addiction: The troublesome concept. Journal of Drug Issues, 21(4), 777793.

Alexander, B. K. (2010). A change of venue for addiction: From medicine to social science. Retrieved 17/12/2017 http://www.brucekalexander.com/articles-speeches/176change-of-venue-1

Alexandrescu, L. (2014). Mephedrone, assassin of youth: The rhetoric of fear in contemporary drug scares. Crime, Media, Culture, 10(1), 23-37.

Allen, C. (2005). The link between heroin, crack cocaine and crime: Where doses street crime fit in? British Journal of Criminology, 45(3), 355-372.

Allman, D., Myers, T., Schellenberg, J., Strike, C., Cockerill, R., \& Cavalieri, W. (2007). Improving health and social care relationships for harm reduction. International Journal of Drug Policy, 18(3), 194-203.

Amon, J., Pearshouse, R., Cohen, J., \& Schleifer, R. (2013). Compulsory drug detention centers in China, Cambodia, Vietnam, and Laos: Health and human rights abuses. Health Human Rights, 15(2), 124-137.

Amon, J. J., Pearshouse, R., Cohen, J. E., \& Schleifer, R. (2014). Compulsory drug detention in East and Southeast Asia: Evolving government, UN and donor responses. International Journal of Drug Policy, 25(1), 13-20.

Andersen, D., \& Jarvinen, M. (2007). Harm reduction- ideals and paradoxes. Nordic Studies on Alcohol and Drugs, 24(3), 235-252.

ASEAN Inter-Parliamentary Assembly. (2015). Country Progress Report - Prevention and combat against drugs and drug-related crimes. Retrieved from ASEAN InterParliamentary Assembly (AIPA): http://www.na.gov.la/files/aifocom11/Doc for AIFOCOM/EXCUTIVE SUMMARY/AIFOCOM11_Country Report.Vietnam_summary 1_pdf 
Ashton, M. (2008). 'The new abstenionists'. Retrieved 11/10/2018, from Druglink Special Insert

http://www.canadianharmreduction.com/sites/default/files/CrawlerResourceServer .pdf

Bachman, R., \& Schutt, R. K. (2012). Fundamentals of research in criminology and criminal justice (3rd ed.). Thousand Oaks, California, USA: Sage.

Bachman, R. D., \& Schutt, R. K. (2017). Fundamentals of research in criminology and criminal justice (4th Ed.). Thousand Oaks, California, USA: Sage.

Bancroft, A. (2009). Drugs, intoxication and society. Cambridge, UK: Polity.

Bankoff, G., \& McCoy, A. W. (2007). The politics of heroin: CIA complicity in the global drug trade. New York, New York, USA: Lawrence Hill Books.

Bauer, R., Eppler, N., \& Wolf, J. (2007). Implications of understandings of addiction: An introductory overview. Bioethics, 7(1), 29-32.

Bayoumi, A. M., \& Zaric, G. S. (2008). The cost-effectiveness of Vancouver's supervised injection facility. Canadian Medical Association Journal, 179(11), 1143-1151. doi:10.1503/cmaj.080808

Bean, P. (2014). Drugs and crime. New York, New York, USA: Routledge.

Becoña, E. (2018). Brain disease or biopsychosocial model in addiction? Remembering the Vietnam Veteran Study. Psicothema, 30(3), 270-275.

Bennett, T. (1998). Drugs and crime: The results of research on drug testing and interviewing arrestees. London, UK: Home Office.

Bennett, T., \& Holloway, K. (2005). Understanding drugs, alcohol, and crime. London, UK: McGraw-Hill Education.

Bennett, T., \& Holloway, K. (2007). Drug-crime connections. Cambridge, UK: Cambridge University Press.

Beresford, M. (2008). Doi Moi in review: The challenges of building market socialism in Vietnam. Journal of Contemporary Asia, 38(2), 221-243. doi:https://doi.org/10.1080/00472330701822314

Bergenstrom, A., Andreeva, V., \& Reddy, A. (2013). Overview of epidemiology of injection drug use and HIV in Asia. Retrieved from Bangkok, Thailand, UNODC: 
https://www.unodc.org/documents/southeastasiaandpacific//poster/Regional over view of IDU and HIV in Asia final 3 Jun 2013.pdf

Berridge, V. (1999). Histories of harm reduction: Illicit drugs, tobacco, and nicotine. Substance Use \& Misuse, 34(1), 35-47.

Berridge, V. (2009). Heroin prescription and history. New England Journal of Medicine, 361(8), 820-821.

Bewley-Taylor, D., \& Jelsma, M. (2012). Regime change: Re-visiting the 1961 Single Convention on Narcotic Drugs. International Journal of Drug Policy, 23(1), 72-81.

Birgin, R. (2013). Arguments against the compulsory treatment of opioid dependence. Bulletin of the World Health Organization, 91(4), 239-239a.

Blalock-Wiker, C. P. (2014). Over the counter care: Service provider perspectives on the application of harm reduction in a syringe exchange program. Long Beach, California, USA: California State University.

Boland, P. (2008). British drugs policy : Problematizing the distinction between legal and illegal drugs and the definition of the ' drug problem'. Probation Journal, 55 (2), 171187.

Borkman, T., Kaskutas, L. A., \& Owen, P. (2007). Contrasting and converging philosophies of three models of alcohol/other drugs treatment: Minnesota model, social model, and addiction therapeutic communities. Alcoholism Treatment Quarterly, 25(3), 21-38.

Borsos, D. (2009). Biopsychosocial model of addiction. In Gary L. Fisher \& N. A. Roget (Eds.), Encyclopedia of substance abuse prevention, treatment, and recovery (1st ed., pp. 27). London, UK: Sage.

Bottoms, A. (1995). The philosophy and politics of punishment and sentencing. In C. Clarkson \& R. Morgan (Eds.), The politics of sentencing reform (1st ed., pp. 17-49). Oxford, UK: Clarendon Press.

Bound, M. (2011). Qualitative method of research: Grounded theory research. Retrieved 20/10/2017, from Nove Southeastern University https://www.academia.edu/1526814/Qualitative Research Grounded Theory Met hodology

Boyatzis, R. E. (1998). Transforming qualitative information: Thematic analysis and code development. Thousand Oaks, California, USA: Sage. 
Boyce, C., \& Neale, P. (2006). Conducting in-depth interviews: A guide for designing and conducting in-depth interviews for evaluation input. Retrieved 20/11/2017, from Pathfinder International https://dmeforpeace.org/sites/default/files/Boyce In Depth $\underline{\text { Interviews.pdf }}$

Bradley, M. (2004). Becoming 'Van Minh': Civilizational discourse and visions of the self in twentieth-century Vietnam. Journal of World History, 15(1), 65-83.

Brady, H. E., \& Collier, D. (2010). Rethinking social inquiry: Diverse tools, shared standards. Plymouth, UK: Rowman \& Littlefield Publishers.

Braun, V., \& Clarke, V. (2006). Using thematic analysis in psychology. Qualitative Research in Psychology, 3(2), 77-101.

Brink, E., Dellve, L., Hallberg, U., Abrahamsson, K. H., Klingberg, G., \& Wentz, K. (2006). Constructing grounded theory. A practical guide through qualitative analysis. International Journal of Qualitative Studies on Health and Well-being, 1(3), 188-192.

Brunt, T. (2017). Drug checking as a harm reduction tool for recreational drug users: Opportunities and challenges. Retrieved from European Monitoring Centre for Drugs and Drug Addictio (EMCDDA):

http://www.emcdda.europa.eu/system/files/attachments/6339/EuropeanResponse sGuide2017 BackgroundPaper-Drug-checking-harm-reduction 0.pdf

Buchanan, J. (2004a). Missing links? Problem drug use and social exclusion. Probation Journal, 51(4), 387-397. doi:10.1177/0264550504048246

Buchanan, J. (2004b). Tackling problem drug use: A new conceptual framework. Social Work in Mental Health, 2(2-3), 117-138.

Buchanan, J. (2006). Understanding problematic drug use: A medical matter or a social issue. British Journal of Community Justice, 4(2), 387-397.

Buchanan, J. (2008). Understanding and engaging with problematic substance use. In Simon Green, Elizabeth Lancaster, \& S. Feasey (Eds.), Addressing offending behaviour Context, practice, values (pp. 246-264). Exeter, Devon: William Publishing.

Buchanan, J. (2009). Understanding and misunderstanding problem drug use: Working together. In Ros Carnwell \& J. Buchanan (Eds.), Effective practice in health, social care \& criminal justice: A partnership approach (pp. 111-128). Reading, Berkshire, UK: Open University Press.

Buchanan, J. (2015a). Drugs and crime. Retrieved 25/05/2018, from Prezi.com https://prezi.com/5du_oyu7x5gy/crim-212-drugs-and-crime-2014/ 
Buchanan, J. (2015b). Ending drug prohibition with a hangover. British Journal of Community Justice, 13(1), 55-74.

Buchanan, J. (2015c). Vested interest is the driver of prohibition. Retrieved 21/10/2017, from https://julianbuchanan.wordpress.com/ https://julianbuchanan.wordpress.com/2015/04/02/15-benefits-from-the-war-ondrugs/

Buchanan, J. (2016a). 70 drug policy fallacies. Retrieved 14/03/2017, from https://julianbuchanan.wordpress.com/

Buchanan, J. (2016b). Are reformers seeking to abolish prohibition or tweak it? Retrieved 17/10/2018, from https://julianbuchanan.wordpress.com/ https://julianbuchanan.wordpress.com/2016/07/08/what-will-drug-reform-deliver/

Buchanan, J., \& Young, L. (2000). The war on drugs or a war on drug users? Drugs: Education, Prevention and Policy, 7(4), 409-422.

Burger, C. A. (2004). Drug abuse, crime, abandoned by bank. Yet Van city was undeterred. Credit Union Journal, 8(28), 43-44.

Carr, E. S. (2010). Scripting addiction: The politics of therapeutic talk and American sobriety. Princeton, New Jersey, USA: Princeton University Press.

Carter, H. R. (1990). The opium war in China: An analysis of Great Britain's use of war as an element of power. Retrieved 31/10/2018 http://www.dtic.mil/dtic/tr/fulltext/u2/a223325.pdf

Caulfield, L., \& Hill, J. (2014). Criminological researchfor beginners - A student's guide. New York, New York, USA: Routledge.

Cavalieri, W., \& Riley, D. (2012). Harm reduction in Canada: The many faces of regression. Retrieved 11/22/2016 https://canadianharmreduction.com/sites/default/files/Harm $\underline{\text { Reduction in Canada.pdf }}$

Chamberlain, J. M. (2013). Understanding criminological research: A guide to data analysis. London, UK: Sage.

Chan, A., Kerkvliet, B. J., \& Unger, J. (1999). Transforming asian socialism: China and Vietnam compared (Vol. 40). Sydney, Australia: Rowman \& Littlefield.

Chang, K. C., \& Lin, C. Y. (2015). Effects of publicly funded and quality of life on attendance rate among methadone maintenance treatment patients in Taiwan: An 18-month follow-up study. Harm Reduction Journal, 12(1), 1-7. doi:10.1186/s12954-015-0076-8 
Charmaz, K. (2006). Constructing grounded theory: A practical guide through qualitative analysis (Introducing qualitative methods series). London, UK: Sage.

Charmaz, K. (2008). Constructionism and the grounded theory method. In J. A. Holstein \& J. F. Gubrium (Eds.), Handbook of constructionist research (pp. 397-412). New York, New York, USA: The Guilford Press.

Charmaz, K. (2011). Grounded theory methods in social justice research. In Norman K. Denzin \& Y. S. Lincoln (Eds.), The Sage handbook of qualitative research (4th ed., Vol. 4, pp. 359-380). New York, New York, USA: Sage.

Charmaz, K. (2014). Constructing grounded theory. New York, New York, USA: Sage.

Chermak, S. (1997). The presentation of drugs in the news media: The news sources involved in the construction of social problems. Justice Quarterly, 14(4), 687-718.

Chomsky, N. (1998). The drug war industrial complex: Noam Chomsky interviewed by John Veit. Retrieved 13/10/2018, from High Times https://chomsky.info/199804 -2/

Choy, L. T. (2014). The strengths and weaknesses of research methodology: Comparison and complimentary between qualitative and quantitative approaches. Journal of Humanities and Social Science, 19(4), 99-104.

Clark, M. (2011). Conceptualising addiction: How useful is the construct? International Journal of Humanities \& Social Science, 1(13), 55-64.

Cohen, J. (2003). HIV and heroin: A deadly international affair: Vietnam. Science, 301(5640), 1657-1658.

Collins, S. E., Clifasefi, S. L., Logan, D. E., Sample, L. S., Somers, J. M., \& Marlatt, G. A. (2012). Current status, historical hightlights, and basic principles of harm reduction. In G. Alan Marlatt, Mary E. Larimer, \& K. Witkiewitz (Eds.), Harm Reduction - Pragmatic strategies for managing highrisk behaviors (2nd ed., pp. 3-36). New York, New York, USA: The Guilford Press.

Cook, C., Bridge, J., McLean, S., Phelan, M., \& Barrett, D. (2014). The funding crisis for harm reduction: Donor retreat, government neglect and the way forward. Retrieved from London, UK, the International Harm Reduction Association:

Coomber, R. (1998). The control of drugs and drug users: Reason or reaction? (Vol. 1). Amsterdam, The Netherlands: CRC Press. 
Coomber, R., Hunt, G., \& Milhet, M. (2016). Social fear, drug related beliefs and drug policy. In Geoffrey Hunt, Maitena Milhet, \& H. Bergeron (Eds.), Drugs and culture: Knowledge, consumption and policy (pp. 15-33). London, UK: Routledge.

Coomber, R., McElrath, K., Measham, F., \& Moore, K. (2013). Key concepts in drugs and society. London, UK: Sage.

Corkery, J. M., Schifano, F., Ghodse, A. H., \& Oyefeso, A. (2004). The effects of methadone and its role in fatalities. Human Psychopharmacology: Clinical and Experimental, 19(8), 565-576.

Costley, C., Elliott, G., \& Gibbs, P. (2010). Doing work based research: Approaches to enquiry for insider-researchers. London, UK: Sage.

Count the Costs. (2010). The war on drugs: Promoting stigma and discrimination. Retrieved from Count the Costs Organisation: http://www.countthecosts.org/sites/default/files/Stigma-briefing.pdf

Creswell, J. W. (2007). Qualitative inquiry \& research design: Choosing among five approaches, . Thousand Oaks,California, USA: Sage.

Creswell, J. W. (2012). Qualitative inquiry and research design: Choosing among five approaches. Thousand Oaks, California, USA: Sage.

Crofts, N., \& Azim, T. (2015). Harm reduction in Asia and the Pacific: an evolving public health response. Harm Reduct J, 12(1), 47. doi:10.1186/s12954-015-0074-x

Csete, J. (2010). From the mountaintops: What the world can learn from drug policy change in Switzerland. Retrieved from The Open Society Foundations, Columbia University: http://pdc.ceu.hu/archive/00006247/01/drugpolicych.pdf

Csete, J., \& Grob, P. J. (2012). Switzerland, HIV and the power of pragmatism: Lessons for drug policy development. International Journal of Drug Policy, 23(1), 82-86. doi:10.1016/j.drugpo.2011.07.011

Csete, J., Kamarulzaman, A., Kazatchkine, M., Altice, F., Balicki, M., Buxton, J., . . . Goulão, J. (2016). Public health and international drug policy. The Lancet, 387(10026), 14271480.

Dao, A., Hirsch, J., Giang, L. M., \& Parker, R. G. (2013). Social science research of HIV in Vietnam: A critical review and future directions. Global Public Health, 8(1), 7-29. doi:10.1080/17441692.2013.811532

Davies, J. B. (2013). Myth of addiction. New York, New York, USA: Routledge. 
Della-Porta, D., \& Keating, M. (2008). Approaches and methodologies in the social sciences: A pluralist perspective. Cambridge, UK: Cambridge University Press.

Denning, P. (2001). Strategies for implementation of harm reduction in treatment settings. Journal of Psychoactive Drugs, 31(1), 23-26.

Denning, P., \& Little, J. (2011). Practicing harm reduction psychotherapy: An alternative approach to addictions. New York, New York, USA: Guilford Press.

Devaney, M., Reid, G., \& Baldwin, S. (2006). Situational analysis of illicit drug issues and responses in the Asia-Pacific region. Retrieved from Australian National Council on Drugs: https://www.burnet.edu.au/system/publication/file/795/ANCD Report Situational analysis of illicit drug issues and responses in the AsiaPacific region.pdf

Dinh, P., \& Duc, T. (2014). As crime, drug use rise, addicts run loose in Ho Chi Minh City. Retrieved 17/10/2017, from Thanh Nien News http://www.thanhniennews.com/society/as-crime-drug-use-rise-addicts-run-loosein-ho-chi-minh-city-32152.html

Doherty, M. C., Junge, B., Rathouz, P., Garfein, R. S., Riley, E., \& Vlahov, D. (2000). The effect of a needle exchange program on numbers of discarded needles: A 2-year follow-up. American Journal of Public Health, 90(6), 936-939.

Dolan, K., Larney, S., \& Wodak, A. (2007). The integration of harm reduction into abstinencebased therapeutic communities: A case study of we help ourselves. Asian Journal Counsel, 14, 1-19.

Domanico, A., \& Malta, M. (2012). Implementation of harm reduction toward crack users in Brazil: Barriers and achievements. Substance Use \& Misuse, 47(5), 535-546.

Duc, N. M. (2012). Phòng chống hành vi vi phạm pháp luật và tệ nạn xã hội với trẻ vị thành niên (English translation: Preventing law breaking behaviours and drug evils among adolescence). Hanoi, Vietnam: People's Police Academy.

Ducan, D. F., Nicholson, T., White, J. B., \& Ellis-Griffith, G. (2014). A brief history of probihition and treatment solution for substance abusers. International Journal of Criminology and Sociology, 3, 186-199.

Duff, C. (2015). Governing drug use otherwise: For an ethics of care. Journal of Sociology, 51(1), 81-96.

Durrant, R., \& Thakker, J. (2003). Substance use and abuse: Cultural and historical perspectives. Thousand Oaks, California, USA: Sage. 
Edington, C. (2016). Drug detention and human rights in post-Doimoi Vietnnam. In J. G. Singh \& D. D. Kim (Eds.), The Postcolonial World (pp. 325-335). London, UK: Routledge.

Edington, C., \& Bayer, R. (2013). When grammars collide: Harm reduction, drug detention and the challenges of international policy reform efforts in Vietnam. Global Public Health, 8(sup1), 75-91.

Eldred-Grigg, S. (1984). Pleasures of the flesh: Sex and drugs in colonial New Zealand, 18401915. Auckland, New Zealand: Reed.

Eliaeson, S. (2002). Max Weber's methodologies: Interpretation and critique. Cambridge, UK: Polity.

Elliott, R., Csete, J., Wood, E., \& Kerr, T. (2005). Harm reduction, HIV/AIDS, and the human rights challenge to global drug control policy. Health and Human Rights, 8(2), 104138.

Engward, H. (2013). Understanding grounded theory. Nursing Standard, 28(7), 37-41.

Epstein, E. J. (1990). Agency of fear: Opiates and political power in America. New York, New York, USA: Verso.

Erceg-Hurn, D. M. (2008). Drugs, money, and graphic ads: A critical review of the Montana Meth Project. Prevention Science, 9(4), 256-263.

Erickson, P. G. (1999). Introduction: The three phases of harm reduction - An examination of emerging concepts, methodologies, and critiques. Substance Use \& Misuse, 34(1), 17.

Erickson, P. G., Riley, D. M., Cheung, Y. W., \& O'Hare, P. A. (1997). Harm reduction: A new direction for drug policies and programs. Toronto, Ontario, Canada: University of Toronto Press.

European Monitoring Centre for Drugs and Drug Addiction. (2017). United Kingdom country drug report 2017. Retrieved from Luxembourg, Publications Office of the European Union:

http://www.emcdda.europa.eu/system/files/publications/4529/TD0116925ENN.pdf

Ezard, N. (2001). Public health, human rights and the harm reduction paradigm: From risk reduction to vulnerability reduction. International Journal of Drug Policy, 12(3), 207219. 
Farisco, M., Evers, K., \& Changeux, J.-P. (2018). Drug Addiction: From Neuroscience to Ethics. Front Psychiatry, 9(595). doi:10.3389/fpsyt.2018.00595

Fedotov, Y. (2010). The unintended negative consequences of the ' war on drugs;: Mass criminalisation and punitive sentencing policies. Retrieved from Penal Reform International Organisation: http://www.penalreform.org/wpcontent/uploads/2013/05/PRI war-on-drugs-briefing March-2013.pdf

Fergusson, D., \& Boden, J. (2011). Cannabis use in adolescence. Retrieved 20/10/2017, from Christchurch Health and Development Study, University of Otago, Christchurch, New Zealand https://www.otago.ac.nz/christchurch/otago018744.pdf

Fernandez, J. (2012). Reducing negative consequences associated with substance misuse within a harm reduction psychotherapy model. (Unpublished PhD thesis), Alliant International University, Los Angeles, California, USA.

Frey, B. S. (1997). Drugs, economics and policy. Economic Policy, 12(25), 388-398.

Gabor, M. (2010). In the realm of hungry ghosts: Close encounters with addiction. Berkeley, California, USA: North Atlantic Books.

Gelkopf, M., Levitt, S., \& Bleich, A. (2002). An integration of three approaches to addiction and methadone maintenance treatment: The self-medication hypothesis, the disease model and social criticism. The Israel journal of psychiatry and related sciences, 39(2), 140.

Gibson, D. R., Brand, R., Anderson, K., Kahn, J. G., Perales, D., \& Guydish, J. (2002). Two-to sixfold decreased odds of HIV risk behavior associated with use of syringe exchange. Journal of Acquired Immune Deficiency Syndromes, 31(2), 237-242.

Giddings, L. S., \& Grant, B. M. (2006). Mixed methods research for the novice researcher. Contemporary Nurse, 23(1), 3-11.

Global Commission on Drug Policy. (2014). Taking control: Pathways to drug policies that work. Retrieved from The Global Commission on Drug Policy:

http://www.globalcommissionondrugs.org/wpcontent/uploads/2016/03/GCDP 2014 taking-control EN.pdf

Global Commission on Drug Policy. (2017). The world drug perception problem: Countering prejudice about people who use drugs. Retrieved from The Global Commission on Drug Policy: http://www.globalcommissionondrugs.org/wpcontent/uploads/2018/01/GCDP-Report-2017 Perceptions-ENGLISH.pdf

Goldberg, T. (1999). Demystifying drugs: A psychosocial perspective. London, UK: Macmillan Press. 
Goldberg, T. (2004). The evolution of Swedish drug policy. The Journal of Drug Issues, 34(3), 551-576.

Goldstein, P. J. (1985). The drugs/violence nexus: A tripartite conceptual framework. Journal of Drug Issues, 15(4), 493-506.

Gossop, M. (2012). Living with drugs (7th ed.). Kingston upon Thames, Surrey, UK: Ashgate.

Gray, D. E. (2009). Doing Research in the Real World. London, UK: Sage.

Gray, D. E. (2013). Doing research in the real world. London, UK: Sage.

Greenwald, G. (2009). Drug decriminalization in Portugal: Lessons for creating fair and successful drug policies. from Cato Institute Whitepaper Series, available at SSRN: https://ssrn.com/abstract=1464837 or http://dx.doi.org/10.2139/ssrn.1464837

Grella, C. E., Anglin, M. D., Wugalter, S. E., Rawson, R., \& Hasson, A. (1994). Reasons for discharge from methadone maintenance for addicts at high risk of HIV infection or transmission. Journal of Psychoactive Drugs, 26(2), 223-232.

Gurstein, P., \& Small, D. (2005). From housing to home: Reflexive management for those deemed hard to house. Housing Studies, 20(5), 717-735.

doi:10.1080/02673030500213953

Gurule, J. (1998). 1988 UN Convention against illicit traffic in narcotic drugs and psychotropic substances -A ten year perspective: Is international cooperation merely illusory? Fordham International Law Journal, 22(1), 74-121.

Ha, P. N., Pharris, A., Huong, N. T., Chuc, N. T. K., Brugha, R., \& Thorson, A. (2010). The evolution of HIV policy in Vietnam: From punitive control measures to a more rightsbased approach. Global Health Action, 3, 1-10.

Hall, W., Carter, A., \& Forlini, C. (2015). The brain disease model of addiction: is it supported by the evidence and has it delivered on its promises? The Lancet Psychiatry, 2(1), 105-110. doi:10.1016/s2215-0366(14)00126-6

Hallberg, L. R. (2006). The "core category" of grounded theory: Making constant comparisons. International Journal of Qualitative Studies on Health and Well-being, 1(3), 141-148.

Hammer, R., Dingel, M., Ostergren, J., Partridge, B., McCormick, J., \& Koenig, B. A. (2013). Addiction: Current criticism of the brain disease paradigm. AJOB Neurosci, 4(3), 2732. doi:10.1080/21507740.2013.796328 
Hammersley, R. (2008). Drugs and crime: Theories and practices (Vol. 2). Cambridge, UK: Polity.

Hammett, T. M., Des Jarlais, D., Johnston, P., Kling, R., Ngu, D., Liu, W., ... Donghua, M. (2007). HIV prevention for injection drug users in China and Vietnam: Policy and research considerations. Global Public Health, 2(2), 125-139.

Hammett, T. M., Wu, Z., Duc, T. T., Stephens, D., Sullivan, S., Liu, W., . . Des Jarlais, D. C. (2008). 'Social evils' and harm reduction: The evolving policy environment for human immunodeficiency virus prevention among injection drug users in China and Vietnam. Addiction, 103(1), 137-145.

Harding, G. (1986). Constructing addiction as a moral failing. Sociology of Health \& IIlness, $8(1), 75-85$.

Hargraves, J. (2015). We live with it almost every day of our lives: An AIVL report into experiences of stigma and discrimination. Retrieved from Australian Injecting and Illicit Drug Users League (AIVL): http://www.aivl.org.au/wpcontent/uploads/2018/06/AIVL-Discrimination-Survey-Results-Report-to-December2015.pdf

Harm Reduction International. (2016). The global state of harm reduction 2016. Retrieved from London, UK: Harm Reduction International: https://www.hri.global/files/2016/11/14/GSHR2016 14nov.pdf

Harris, J., \& McElrath, K. (2012). Methadone as social control institutionalized stigma and the prospect of recovery. Qualitative Health Research, 22(6), 810-824.

Harris, S. (2012). Out of harm's way: The politics and practice of harm reduction in Argentina. (Unpublished PhD Thesis), University of California, San Francisco, California, USA.

Harrison, L. D., Backenheimer, M., \& Inciardi, J. A. (1995). Cannabis use in the United States: Implications for policy. Retrieved from Center for Drug and Alcohol Studies, University of Delaware, USA:

http://citeseerx.ist.psu.edu/viewdoc/download?doi=10.1.1.737.1516\&rep=rep1\&typ $\underline{e=p d f}$

Hart, C. (2013). High price: A neuroscientist's journey of self-discovery that challenges everything you know about drugs and society. New York, New York, USA: Harper Collins.

Hart, C. L. (2017). Viewing addiction as a brain disease promotes social injustice. Retrieved 13/10/2018, from Nature Human Behaviour https://www.nature.com/articles/s41562-017-0055 
Hartney, E. (2019). DSM 5 Criteria for Substance Use Disorders. Retrieved 23/04/2019, from www.verywellmind.com https://www.verywellmind.com/dsm-5-criteria-forsubstance-use-disorders-21926

Hathaway, A. D. (2001). Shortcomings of harm reduction: Toward a morally invested drug reform strategy. International Journal of Drug Policy, 12(2), 125-137.

Hayes-Larson, E., Grau, L. E., Khoshnood, K., Barbour, R., Khuat, O. T. H., \& Heimer, R. (2013). Drug users in Hanoi, Vietnam: Factors associated with membership in community-based drug user groups. Harm Reduction Journal, 10(33), 1-11.

Hendin, H. (1980). Psychosocial theory of drug abuse: A psychodynamic approach. In D. Lettieri , M. Sayers, \& H. Pearson (Eds.), Theories on drug abuse (Vol. 30, pp. 195203). Rockville, Maryland, USA: National Institute on Drug Abuse.

Heyman, G. M. (2009). Addiction: A disorder of choice: Harvard University Press.

Heyman, G. M. (2013). Addiction and choice: Theory and new data. Front Psychiatry, 4, 31. doi:10.3389/fpsyt.2013.00031

Hilal, A. H., \& Alabri, S. S. (2013). Using Nvivo for data analysis in qualitative research. International Interdisciplinary Journal of Education, 2(2), 181-186.

Hill, D. A. (2005). A case study approach to the leadership ascension and the paths to power in Liberia. (Unpublished PhD Thesis), University of Phoenix, Phoenix, Arizona, USA.

Hilton, B., Thompson, R., Moore-Dempsey, L., \& Janzen, R. G. (2001). Harm reduction theories and strategies for control of human immunodeficiency virus: A review of the literature. Journal of Advanced Nursing, 33(3), 357-370.

Hoang, T. V., Ha, T. T., Hoang, T. M., Nhu, N. T., Quoc, N. C., Tam, N. T., \& Mills, S. (2015). Impact of a methadone maintenance therapy pilot in Vietnam and its role in a scaled-up response. Harm Reduction Journal, 12(1), 39. doi:10.1186/s12954-0150075-9

Hoe, H. D. (2013). Yếu tố tác động đến các chương trình Methadone ở thành phố Hồ Chí Minh (English translation: Impacting Factors on Methadone Programmes in Ho Chi Minh City). (Unpublished MBA Thesis), University of Social Science and Humanities, Ho Chi Minh, Vietnam.

Hofschulte, R. (2012). Clinical social workers' beliefs towards harm reduction when working with substance using clients. (Unpublished MBA Thesis), St. Catherine University, Minnesota, USA. Retrieved from http://sophia.stkate.edu/msw papers/37 
Holloway, K., Bennett, T., \& Farrington, D. P. (2005). The effectiveness of criminal justice and treatment programmes in reducing drug-related crime: A systematic review (1844736385). Retrieved from Research Development and Statistics Directorate, Home Office, London, UK:

https://pdfs.semanticscholar.org/c102/f6dc524df3539eb69b03f1263481bacf3efa.pd $\underline{f}$

Hong, K. T., Nguyen, A. T. V., \& Ogden, J. (2004). "Because this is the disease of the century": Understanding HIV and AIDS-related stigma and discrimination in Vietnam. Retrieved from International center for research on women (ICRW), Hanoi, Vietnam:

https://www.icrw.org/wp-content/uploads/2016/10/Understanding-HIV-and-AIDSrelated-Stigma-and-Discrimination-in-Vietnam.pdf

Hong, K. T., Van Anh, N. T., Oanh, K. T. H., Kidd, R., Brady, L., Nyblade, L., \& Stangl, A. (2011). Understanding and challenging stigma toward injecting drug users and HIV in Vietnam. Toolkit for action. Retrieved from Institute for Social Development Studies and International Center for Research on Women, Hanoi, Vietnam:

https://www.icrw.org/wp-content/uploads/2016/10/Toolkit-for-action---Reducingstigma-toward-drug-addiction-and-HIV-in-Vietnam.PDF

Horvath, M. A., \& LeBoutillier, N. (2014). Alcohol use and crime. (1118517385). Retrieved 04/10/2017, from The Encyclopedia of Criminology and Criminal Justice https://search.credoreference.com/content/entry/wileycacj/alcohol use and crime $\angle 0$

Hughes, C. E., \& Stevens, A. (2010). What can we learn from the Portuguese decriminalization of illicit drugs? British Journal of Criminology, 50(6), 999-1022.

Human Rights Watch. (2011). The rehab archipelago - Forced labor and other abuses in drug detention centers in Southern Vietnam. Retrieved from Human Rights Watch: http://www.hrw.org/sites/default/files/reports/vietnam0911ToPost.pdf

Human Rights Watch. (2012). Tortue in the name of treatment - Human right abuses in Vietnam, China, Cambodia and Laos. Retrieved from Human Rights Watch: https://www.hrw.org/report/2012/07/24/torture-name-treatment/human-rightsabuses-vietnam-china-cambodia-and-lao-pdr

Hunt, N., Ashton, M., Lenton, S., Mitcheson, L., Nelles, B., \& Stimson, G. (2003). A review of the evidence-base for harm reduction approaches to drug use. Retrieved 10/10/2015, from Neil Hunt Organisation http://www.forward-thinking-ondrugs.org/review2-print.html

Huo, D., Bailey, S. L., Hershow, R. C., \& Ouellet, L. (2005). Drug use and HIV risk practices of secondary and primary needle exchange users. Aids Education \& Prevention, 17(2), 170-184. 
Huong, N. V. T. (2002). Governing sex: Medicine and governmental intervention in prostitution. In J. Werner \& D. Belanger (Eds.), Gender, Household, State: Doi Moi in Vietnam (Vol. 3). New York, New York, USA: Cornell University.

Hutton, F. (2016). Legal highs and their use in New Zealand: A critical analysis of New Zealand drug policy. Prison Service Journal, 227(10), 29-37.

Hutton, F. (2017). BZP-'Party pills', populism and prohibition: Exploring global debates in a New Zealand context. Australian \& New Zealand Journal of Criminology, 50(2), 282306.

Inciardi, J. A. (1991). The drug legalization debate. Newbury Park, California, USA: Sage.

Inciardi, J. A., \& Harrison, L. D. (1999). Harm reduction: National and international perspectives. London, UK: Sage.

International Harm Reduction Association. (2016). The global state of harm reduction 2016. Retrieved from The International Harm Reduction Association: https://www.hri.global/files/2016/11/14/GSHR2016 14nov.pdf

International Labour Rights Forum. (2014). Vietnam forced labor centers. Retrieved from International Labour Rights Forum: https://laborrights.org/sites/default/files/publications/VN Forced Labor Centers P RINT.pdf

International Network of People Who Use Drugs. (2014). Drug user peace initiative stigmatising people who use drugs. Retrieved from International Network of People Who Use Drugs:

https://www.unodc.org/documents/ungass2016/Contributions/Civil/INPUD/DUPIStigmatising People who Use Drugs-Web.pdf

Johnson, B. (1993). Freedom and interpretation. Oxford, UK: Basic Books.

Johnson, S. (2014). Advantages \& disadvantages of positivism. Retrieved 6/9/2018 https://classroom.synonym.com/advantages-disadvantages-positivism$\underline{12088541 . h t m l}$

Judger, N. (2016). The thematic analysis of interview data: An approach used to examine the influence of the market on curricular provision in Mongolian higher education institutions. Retrieved 20/11/2016, from University of Leeds, Leeds, UK http://hpp.education.leeds.ac.uk/wp-content/uploads/sites/131/2016/02/HPP20163-Jugder.pdf 
Kamarulzaman, A., \& McBrayer, J. L. (2015). Compulsory drug detention centers in East and Southeast Asia. International Journal of Drug Policy, 26, Supplement 1, S33-S37. doi:http://dx.doi.org/10.1016/j.drugpo.2014.11.011

Kamarulzaman, A., \& Saifuddeen, S. (2010). Islam and harm reduction. International Journal of Drug Policy, 21(2), 115-118.

KCBA. (2005). Drugs and the drug laws: Historical and cultural contexts. Retrieved from King County Bar Association Drug Policy Project (KCBA), Seattle, USA: https://www.kcba.org/druglaw/pdf/report hc.pdf

Keane, H. (2003). Critiques of harm reduction, morality and the promise of human rights. International Journal of Drug Policy, 14(3), 227-232. doi:10.1016/s09553959(02)00151-2

Kellehear, A. (1993). The unobtrusive researcher: A guide to methods. Sydney, New South Wales, Australia: Allen \& Unwin.

Kermode, M., Crofts, N., Kumar, M. S., \& Dorabjee, J. (2011). Opioid substitution therapy in resource-poor settings. Bulletin of the World Health Organization, 89(4), 243-243.

Kerr, T., Small, W., \& Wood, E. (2005). The public health and social impacts of drug market enforcement: A review of the evidence. International Journal of Drug Policy, 16(4), 210-220.

Khoi Do, Victor Minichiello, \& Hussain, R. (2012). HIV risks among injecting drug users in Vietnam: A review of the research evidence. Current HIV Research, 10(6), 497-486.

Khuat, T. H., Nguyen, V. A. T., Jardine, M., Moore, T., Bui, T. H., \& Crofts, N. (2012). Harm reduction and "clean" community: Can Vietnam have both? Harm Reduction Journal, 9(25), 1-10.

Kincaid, H., \& Sullivan, J. A. (2010). 13 medical models of addiction. In Don Ross, Harold Kimcaid, David Superett, \& P. Collins (Eds.), What is addiction? (pp. 353-376). Boston, Massachusetts, USA: A Bradford Book.

Kleiman, M. A. R., Caulkins, J. P., \& Hawken, A. (2011). Drugs and drug policy. Oxford, UK: Oxford University Press.

Koop, C. (2003). Drug addiction in America: Challenges and opportunities. Military medicine, 168(5), viii-xvi.

Kuzmarov, J. (2013). The myth of the'addicted army': Drug use in Vietnam in historical perspective. War \& Society, 26(2), 121-141. 
Kyser, N. M. (2010). Counselor attitudes toward the harm reduction approach in substance abuse treatment. (Unpublished PhD Thesis), Old Dominion University, Virginia, USA.

Lancaster, K., Hughes, C., Spicer, B., Matthew-Simmons, F., \& Dillon, P. (2011). Curiosity killed the M-cat: An examination of illicit drugs and media. Paper presented the Australian and New Zealand Critical Criminology Conference 2010, Sydney, Australia. https://ses.library.usyd.edu.au//bitstream/2123/7382/1/Lancaster et al ANZCCC2010.pdf

Lanier, M. M., \& Briggs, L. T. (2014). Research methods in criminal justice and criminology. Oxford, UK: Oxford University Press.

Lau, H. V. (1993). Lịch sử phòng chống ma túy ở Việt Nam (English translation: History of drug prevention in Vietnam). Hanoi, Vietnam: Sunday People.

Le, M. G., Ngoc, L. B., Hoang, V. H., Mulvey, K., \& Rawson, R. A. (2013). Substance use disorders and HIV in Vietnam since Doi Moi (Renovation): An overview. Journal of Food and Drug Analysis, 21(4), S42-S45.

Lenton, S., \& Single, E. (1998). The definition of harm reduction. Drug and Alcohol Review, $17(2), 213-219$.

Leshner, A. I. (1997). Addiction is a brain disease, and it matters. Science, 278(5335), 45-47.

Levi, R., \& Appel, J. (2003). Collateral consequences: Denial of basic social services based upon drug use. Berkeley, California, USA: Office of Legal Affairs, Drug Policy Alliance.

Levine, H. G. (2002). The secret of worldwide drug prohibition: The varieties and uses of drug prohibition. The independent review, 7(2), 165-180.

Levine, H. G. (2009). Review of "the globalisation of addiction: A Study in poverty of the spirit" by Bruce K. Alexander. Harm Reduction Journal, 6, 12-16.

Levy, J. (2014). The harms of drug use: Criminalisation, misinformation, and stigma. Retrieved from Internationa Network of People Who Use Drug: http://www.inpud.net/The Harms of Drug Use JayLevy2014 INPUD YouthRISE.p $\underline{\mathrm{df}}$

Lewis, M. (2015). The biology of desire: Why addiction is not a disease. New York, New York, USA: Public Affairs Books.

Lindesmith, A. R. (1965). The addict and the law. Indianapolis, Indiana, USA: Indiana University Press. 
Lishchynski, M. A. (2004). Harm reduction from the perspective of the illicit drug user: $A$ study. (Unpublished MBA Thesis), The Department of Criminology, University of Ottawa, Ottawa, Canada. Retrieved from https://www.ruor.uottawa.ca/bitstream/10393/26958/1/MR11328.PDF

Luong, H. T. (2017). Transnational Narcotics Trafficking across Vietnam Borderland and Lao People's Democratic Republic. München, Germany: GRIN Verlag.

Lynch, M., \& Bogen, D. (1997). Sociology's asociological" core": An examination of textbook sociology in light of the sociology of scientific knowledge. American Sociological Review, 62(3), 481-493.

MacBride, K. (2017). This 38-year-old study is still spreading bad ideas about addiction. Retrieved 24/04/2019, from theoutline.com https://theoutline.com/post/2205/this38-year-old-study-is-still-spreading-bad-ideas-about-addiction

MacCoun, R. J., \& Reuter, P. (2001). Drug war heresies: Learning from other vices, times, and places. Cambridge, UK: Cambridge University Press.

MacMaster, S. A. (2004). Harm reduction: A new perspective on substance abuse services. Social Work, 49(3), 356-363. doi:10.1093/sw/49.3.353

Maher, L., Coupland, H., \& Musson, R. (2007). Scaling up HIV treatment, care and support for injecting drug users in Vietnam. International Journal of Drug Policy, 18(4), 296305.

Maltby, L. (2001). Drug testing: A bad investment. Business Ethics: The Magazine of Corporate Responsibility, 15 (2), 7-7.

Marlatt, G. A. (2000). Harm reduction: Basic principles and strategies. The Prevention Researcher, 7(2), 1-4.

Marlatt, G. A., Marry, E. L., \& Witkiewitz, K. (2012). Harm reduction - Pragmatic strategies for managing high-risk behaviors. New York, New York, USA: Guilford Press.

Marlatt, G. A., \& Witkiewitz, K. (2010). Update on harm-reduction policy and intervention research. Annual Review of Clinical Psychology, 6, 591-606.

Marshall, Z., Dechman, M. K., Minichiello, A., Alcock, L., \& Harris, G. E. (2015). Peering into the literature: A systematic review of the roles of people who inject drugs in harm reduction initiatives. Drug Alcohol Dependence, 151, 1-14.

doi:10.1016/j.drugalcdep.2015.03.002 
Martin, C. S., Chung, T., \& Langenbucher, J. W. (2008). How should we revise diagnostic criteria for substance use disorders in the DSM-V? Journal of Abnormal Psychology, $117(3), 561$.

Maxwell, J. A., \& Mittapalli, K. (2010). Realism as a stance for mixed methods research. In A. Tashakori \& C. Teddie (Eds.), Handbook of mixed methods in social and behavioral research (2nd ed., pp. 145-168). London, UK: Sage.

McCoy, A. W., Read, C. B., \& Adams, L. P. (1972). The politics of heroin in Southeast Asia. New York, New York, USA: Harper Colophon Books.

McKeganey, N. (2011). Abstinence and harm reduction: Can they work together? International Journal of Drug Policy, 22(3), 194-195.

McKenna, T. (1999). Food of the gods: The search for the original tree of knowledge: $A$ radical history of plants, drugs, and human evolution. Auckland, New Zealand: Random House.

McLellan, A. T., Lewis, D. C., O'Brien, C. P., \& Kleber, H. D. (2000). Drug dependence, a chronic medical illness: Implications for treatment, insurance, and outcomes evaluation. Jama, 284(13), 1689-1695.

McNeece, A. C. (2003). After the war on drugs is over: Implications for social work education. Journal of Social Work Education, 39(2), 193-212.

Menninger, R. W., \& Nemiah, J. C. (2008). American psychiatry after World War II (19441994). Washington, DC, USA: American Psychiatric.

Miller, P. G. (2001). A critical review of the harm minimization ideology in Australia. Critical Public Health, 11(2), 167-178.

Miller, W. R. (2002). Motivational interviewing : Preparing people for change. New York, New York, USA: Guilford Press.

Miller, W. R., \& Rollnick, S. (2012). Motivational interviewing: Helping people change. New York, New York, USA: Guilford Press.

Ministry of Health. (2006). HIV/AIDS in Vietnam: The current situation, the national response, the emerging challenges. Retrieved from Ministry of Health, Hanoi, Vietnam: https://assets.prb.org/pdf07/VietnamHIVChartbook.pdf

Ministry of Health. (2016a). Báo cáo: Công tác phòng, chống HIV/AIDS năm 2015 và các nhiệm vụ trọng tâm năm 2016 (English Translation: The report on HIV/AIDS prevention in 2015 and the main targets for 2016). Retrieved from Ministry of 
Health, Hanoi, Vietnam:

http://vaac.gov.vn/Cms Data/Contents/Vaac/Folders/Solieubaocao/Solieu/ content

s/BCG2DGP6NQ77KBCX/Bao-cao-HIV AIDS-nam-2015-va-nhiem-vu-trong-tam-nam-

2016 final.pdf

Ministry of Health. (2016b). Hỏi và đáp: Phòng chống tác hại của rượi bia (English translation: Questions and answers: Preventing the danger and harm of alcohol). Hanoi, Vietnam: Medical Publisher.

Ministry of Health. (2018a). Báo cáo về đánh giá tác động của chính sách pháp luật trong phòng chống tác bại của rượi, bia và thuốc lá (English translation: Report on evaluating effects of legall frameworks on preventing harm caused by alcohol and tobacco). Retrieved from Ministry of Health, Vietnam: http://duthaoonline.quochoi.vn/DuThao/Lists/DT TAILIEU COBAN/Attachments/32 10/6. Bao cao danh gia tac dong.pdf

Ministry of Health. (2018b). Công tác phòng, chống HIV/AIDS năm 2017 và các nhiệm vụ trọng tâm năm 2018 (English translation: HIV prevention situation in 2017 and targets for 2018). Retrieved from Department of HIV/AIDS Prevention, Ministry of Health, Vietnam: http://vaac.gov.vn/solieu/Detail/Bao-cao-cong-tac-phong-chongHIV-AIDS-nam-2017-va-nhiem-vu-trong-tam-nam-2018

MOLISA. (2015). Tổng quan về nghiện ma túy (English translation: An overiew on drug addiction). Hanoi, Vietnam: Ministry of Labour, Invalids and Social Affairs.

Monaghan, G., \& Bewley-Taylor, D. (2013). Police support for harm reduction policies and practices towards people who inject drugs. Retrieved from London, UK: International Drug Policy Consortium: https://www.grea.ch/sites/default/files/mdle-report1 police-support-for-harm-reduction.pdf

Morales, J. A. (2013). The 'middle-income effect' on HIV/AIDS programs in Vietnam. Retrieved 12/10/2016 https://www.devex.com/news/the-middle-income-effect-onhiv-aids-programs-in-vietnam-81491

MPS. (2015). Báo cáo về công tác phòng chống ma túy năm 2015 và mục tiêu năm 2016 (English translation: The report on drug use prevention in 2015 and the targets for 2016). Retrieved from Hanoi, Vietnam: Ministry of Public Security:

Mueller, S. R., Calcaterra, S. L., Glanz, J. M., \& Binswanger, I. A. (2015). A review of opioid overdose prevention and naloxone prescribing: Implications for translating community programming into clinical practice. Substance abuse, 36(2), 240-253.

Muncie, J. (2010). Labelling, social reaction and social constructionism. In E. McLaughlin \& T. Newburn (Eds.), The Sage handbook of criminological theory (pp. 139-152). London, UK: Sage. 
Ngo, V. (2008). Addiction: According to moral, disease, and learning Models. Retrieved 10/12/2016, from Simon Fraser University, British Columbia, Canada http://www.sfu.ca/ palys/321OralHistory-NgoAddictionAccordingToThreeModels.pdf

Nguyen, T. A., Pauline Oosterhoff, Yen Pham Ngoc, Pamela Wright, \& Hardon, A. (2009). Self-help groups can improve utilization of postnatal care by HIV-infected mothers. Journal of the Association of Nurses in AIDS Care, 20(2), 141-152. doi:10.1016/j.jana.2008.10.006

Nguyen, T. H., \& Wolffers, I. (1994). HIV infection in Vietnam. The Lancet, 343(8894), 367428.

Nguyen, T. P. (2002). Giáo trình về phòng chống nghiện ma túy (English translation: Textbook on drug addiction prevention). Hanoi, Vietnam: The People's Police Academy.

Nguyen, T. P. H. (2007). Legislative implementation by Vietnam of its obligations under the United Nations drug control conventions. (Unpublished PhD Thesis), The University of Wollongong, Wollongong, New South Wales, Australia.

Nguyen, T. V. (2008). Drug abuse among young adults in Vietnam: The influence of family, peers, and society. (Unpublished PhD Thesis), The University of Texas, Arlington, Texas, USA.

Nguyen, T. V., \& Scannapieco, M. (2008). Drug abuse in Vietnam: A critical review of the literature and implications for future research. Addiction, 103(4), 535-543.

Nutt, D. (2012). Drugs - Without the hot air. London: UIT Cambridge Ltd.

Nutt, D., King, L. A., \& Philips, L. D. (2010). Drug harm in the UK: a multicriteria decision analysis. 376, 1558-1565.

Nutt, D., King, L. A., Saulsbury, W., \& Blakemore, C. (2007). Development of a rational scale to assess the harm of drugs of potential misuse. The Lancet, 369(9566), 1047-1053.

O'Hare, Newcombe, R., \& Matthews, A. (1992). The reduction of drug-related harm: A conceptual framework for theory, practice and research. London, UK: Routledge.

O'Malley, P., \& Valverde, M. (2004). Pleasure, freedom and drugs: The uses of 'pleasure'in liberal governance of drug and alcohol consumption. Sociology, 38(1), 25-42.

Orford, J. (2001). Excessive appetites: A psychological view of addictions (2nd Ed.). Chichester, West Sussex, UK: John Wiley \& Sons. 
Parkin, S. (2014). An applied visual sociology: Picturing harm reduction. London, UK: Ashgate Publishing Limited.

Peele, S. (2000). What addiction is and is not: The impact of mistaken notions of addiction. Addiction Research, 8(6), 599-607.

Peele, S. (2012a). AA and abstinence as prevention techniques. Drugs: Education, Prevention and Policy, 19(4), 284-290. doi:10.3109/09687637.2012.671861

Peele, S. (2012b). Addiction myths. RSA Journal, 158(5549), 21-24.

Petersen, A., \& Lupton, D. (1996). The new public health: Health and self in the age of risk. London, UK: Sage.

Pettus, A. (2004). Between sacrifice and desire: National identity and the governing of femininity in Vietnam. New York, New York, USA: Routledge.

Pham, N. H., Pharris, A., Huong, N. T., Chuc, N. T. K., Brugha, R., \& Thorson, A. (2010). The evolution of HIV policy in Vietnam: From punitive control measures to a more rightsbased approach. Global Health Action, 3, 1-10.

Phinney, H. M. (2008). "Rice is essential but tiresome; you should get some noodles": Doi Moi and the political economy of men's extramarital sexual relations and marital HIV risk in Hanoi, Vietnam. American Journal of Public Health, 94(4), 650-660. doi:http://doi.org/10.2105/AJPH.2007.111534

Quang, N. M. (2009). Buôn lậu ma túy ở Việt Nam trước năm 1975 (English translation: Drug trafficking in Vietnam before 30th April 1975). Ho Chi Minh, Vietnam: Giao Hoi La Ma-Vatican.

Race, K. (2008). The use of pleasure in harm reduction: Perspectives from the history of sexuality. International Journal of Drug Policy, 19(5), 417-423.

Ramaswamy, A. (2013). The law of unintended consequences - The Harrison Act: Turning point in America's relationship with drugs. Retrieved 05/04/ 2017, from Aditi's Blog http://thehistoryhacker.com/2013/08/12/the-law-of-unintended-consequences-theharrison-act-turning-point-in-americas-relationship-with-drugs/

Rapin, A.-J., Dao, H. K., \& Pham, H. T. (2003). Ethnic minorities, drug use and harm in the highlands of Northern Vietnam: A contextual analysis of the situation in six communes from Son La, Lai Chau, and Lao Cai. Retrieved from Hanoi, Vietnam, UNODC:

https://www.unodc.org/pdf/vietnam/vietnam ethnic minorities report.pdf 
Rastegar, D. A., \& Fingerhood, M. I. (2005). Addiction medicine: An evidence-based handbook. Philadelphia, Pennsylvania, USA: Lippincott Williams \& Wilkins.

Reid, G., \& Higgs, P. (2011). Vietnam moves forward with harm reduction: An assessment of progress. Global Public Health, 6(2), 168-180.

Rhodes, T., \& Hedrich, D. (2010). EMCDDA monographs - Harm reduction: Evidence, impacts and challenges. Retrieved from The European Monitoring Centre for Drugs and Drug Addiction (EMCDDA):

http://www.emcdda.europa.eu/system/files/publications/555/EMCDDAmonograph10-harm reduction final 205049.pdf

Richard, P., \& Riley, D. (2012). Harm reduction in substabce use and high-risk behaviour international policy and practice. London, UK: Blackwell Publishing.

Richard, T. (2013). Qualitative versus quantitative methods: Understanding why qualitative methods are superior for criminology and criminal justice. Journal of Theoretical and Philosophical Criminology, 1(1), 38-58.

Riley, D., \& O'Hare, P. (2000). Harm reduction: History, definition, and practice. In I. James A (Ed.), Harm reduction: National and international perspectives (pp. 1-26). London, UK: Sage.

Riley, D., Sawka, E., Conley, P., Hewitt, D., Mitic, W., Poulin, C., .. Topp, J. (1999). Harm reduction: Concepts and practice. A policy discussion paper. Substance Use \& Misuse, 34(1), 9-24.

Riordan, K. (2017). The connection between drug use and crime in Western Australia. (Unpublished PhD Thesis), Edith Cowan University, Perth, Australia. Retrieved from http://ro.ecu.edu.au/cgi/viewcontent.cgi?article=2975\&context=theses

Ritter, A., \& Cameron, J. (2006). A review of the efficacy and effectiveness of harm reduction strategies for alcohol, tobacco and illicit drugs. Drug and Alcohol Review, 25(6), 611624.

Ritter, A., Lancaster, K., Grech, K., \& Reuter, P. (2011). An assessment of illicit drug policy in Australia (1985 to 2010): Themes and trends (0733429912). Retrieved from National Drug and Alcohol Research Centre, Sydney, Australia: https://ndarc.med.unsw.edu.au/sites/default/files/ndarc/resources/21 An assessment of illicit drug policy in Australia.pdf

Roberts, M., Klein, A., \& Trace, M. (2004). Towards a review of global policies on illegal drugs. Retrieved from Beckley Foundation Drug Policy Programme: http://archive.beckleyfoundation.org/pdf/report globalpolicy.pdf 
Roe, G. (2005). Harm reduction as paradigm: Is better than bad good enough? The origins of harm reduction. Critical Public Health, 15(3), 243-250.

Rolles, S. (2012). The alternative world drug report: Counting the costs of the war on drugs (0955642833). Retrieved from Count the Costs: http://countthecosts.org/sites/default/files/AWDR.pdf

Roy, A., \& Buchanan, J. (2015). The paradoxes of recovery policy: Exploring the impact of austerity and responsibilisation for the citizenship claims of people with drug problems. Social Policy \& Administration, 50(3), 1-16. doi:10.1111/spol.12139

Rudgley, R. (1994). Essential substances: A cultural history of intoxicants in society. London, UK: Arktos Media.

Rydstrøm, H. (2006). Sexual desires and 'social evils': young women in rural Vietnam. Gender, Place \& Culture, 13(3), 283-301.

Satcher, D. (2000). Evidence-based findings on the efficacy of syringe exchange programs: an analysis of the scientific research completed since April 1998. Retrieved from Department of Health and Human Services - Washington, DC, USA: https://harmreduction.org/wpcontent/uploads/2012/01/EvidenceBasedFindingsOnEfficacyofSEPs.pdf

Satel, S. (1999). Drug treatment: The case for coercion. Washington, DC, USA: AEI Press.

Satel, S., \& Lilienfeld, S. O. (2010). Singing the brain disease blues. AJOB Neurosci, 1(1), 4647. doi:10.1080/21507740903504368

Satel, S., \& Lilienfeld, S. O. (2013). Addiction and the brain-disease fallacy. Front Psychiatry, $4,141$.

Saunders, J. B. (2017). Substance use and addictive disorders in DSM-5 and ICD 10 and the draft ICD 11. Current Opinion in Psychiatry, 30(4), 227-237.

Schaler, J. A. (1991). Drugs and free will. Society, 28(6), 42-49.

Schatz, E., \& Nougier, M. (2012). Drug consumption rooms: Evidence and practice. Retrieved from International Drug Policy Consortium, London, UK: http://www.drugsandalcohol.ie/17898/1/IDPC-Briefing-Paper Drug-consumptionrooms.pdf

Schutt, R. K. (2012). Investigating the social world: The process and practice of research. Thousand Oaks, California, USA: Sage. 
Seddon, T. (2007). Coerced drug treatment in the criminal justice system: Conceptual, ethical and criminological issues. Criminology and Criminal Justice, 7(3), 269-286.

Seddon, T., Ralphs, R., \& Williams, L. (2008). Risk, security and the 'criminalization'of British drug policy. British Journal of Criminology, 48(6), 818-834.

Shelly, S. (2016). The harm reduction movement needs to rediscover its soul. Retrieved 09/12/2017, from Theinfluence.org http://theinfluence.org/the-harm-reductionmovement-needs-to-rediscover-its-soul/ - disqus thread

Shewan, D., \& Dalgarno, P. (2005). Evidence for controlled heroin use? Low levels of negative health and social outcomes among non-treatment heroin users in Glasgow (Scotland). British Journal of Health Psychology, 10(1), 33-48.

Siegler, M., \& Osmond, H. (1968). Models of drug addiction. International Journal of the Addictions, 3(1), 3-24.

Small, D., Palepu, A., \& Tyndall, M. W. (2006). The establishment of North America's first state sanctioned supervised injection facility: A case study in culture change. International Journal of Drug Policy, 17(2), 73-82. doi:10.1016/j.drugpo.2005.08.004

Smith, J. A. (2015). Qualitative psychology: A practical guide to research methods. London, UK: Sage.

Stafford, N. (2007). Using words: The harm reduction conception of drug use and drug users. International Journal of Drug Policy, 18(2), 88-91.

Stevens, A. (2007). When two dark figures collide: Evidence and discourse on drug-related crime. Critical Social Policy, 27(1), 77-99.

Stevens, A. (2010). Drugs, crime and public health: The political economy of drug policy. New York, New York, USA: Routledge.

Stevens, A. (2012). The ethics and effectiveness of coerced treatment of people who use drugs. Human Rights and Drugs, 2, 7-16.

Stevens, A., Bewley-Taylor, D., \& Dreyfus, P. (2009). Drug markets and urban violence: Can tackling one reduce the other? Retrieved from The beckley Foundation Drug Policy Programme: http://beckleyfoundation.org/wpcontent/uploads/2016/04/BF Report 15.pdf

Stevens, A., \& Measham, F. (2014). The 'drug policy ratchet': Why do sanctions for new psychoactive drugs typically only go up? Addiction, 109(8), 1226-1232. 
Stevens, A., Stöver, H., \& Brentari, C. (2010). Criminal justice approaches to harm reduction in Europe. In T. Rhodes \& D. Hedrich (Eds.), Harm reduction: Evidence, impacts and challenges (pp. 379-402). Lisbon, Portugal: Centre for Drugs and Drug Addiction (EMCDDA).

Stimson, G. V. (2007). "Harm Reduction-Coming of Age": A local movement with global impact. International Journal of Drug Policy, 18(2), 67-69.

Strang, J., Groshkova, T., \& Metrebian, N. (2012). New heroin-assisted treatment: Recent evidence and current practices of supervised injectable heroin treatment in Europe and beyond. Luxembourg: Publications Office of the European Union.

Strathdee, S. A., Patrick, D. M., Currie, S. L., Cornelisse, P. G., Rekart, M. L., Montaner, J. S., . . . O'Shaughnessy, M. V. (1997). Needle exchange is not enough: Lessons from the Vancouver injecting drug use study. Rapid Science Publishers, 11(8), 59-65.

Tammi, T., \& Hurme, T. (2007). How the harm reduction movement contrasts itself against punitive prohibition. International Journal of Drug Policy, 18(2), 84-87.

Tatarsky, A. (2007). Harm reduction psychotherapy: A new treatment for drug and alcohol problems. New York, New York, USA: Jason Aronson.

Taylor, S. (2008). Outside the outsiders: Media representations of drug use. Probation Journal, 55(4), 369-387.

Taylor, S., Buchanan, J., \& Ayres, T. (2016). Prohibition, privilege and the drug apartheid: The failure of drug policy reform to address the underlying fallacies of drug prohibition. Criminology \& Criminal Justice, 16(4), 452-469.

Teddlie, C., \& Tashakkori, A. (2010). Overview of contemporary issues in mixed methods research. In A. Tashakkori \& C. Teddlie (Eds.), Handbook of mixed methods in social and behavioral research (pp. 1-41). Thousand Oaks, California, USA: Sage.

Tettey, J., Wong, Y., Levissianos, S., Eichinger, N., Soe, T., Kelley, S., ... Umapornsakula, A. (2013). Patterns and trends of amphetamine-type stimulants and other drugs: Challenges for Asia and the Pacific. Retrieved from Tthe Global SMART Programme, UNODC:

https://www.unodc.org/documents/scientific/2013 Regional ATS Report web.pdf

The National Committee on AIDS Drugs and Prostitution Prevention and Control. (2011). Posters for Drug Use and HIV/AIDS Prevention. Retrieved 14/07/2016, from The National Committee on AIDS Drugs and Prostitution Prevention and Control (Vietnam) http://tiengchuong.vn/gallery/100/Tranh-co-dong-phong-chongHIVAIDS/album83.vgp 
Thien, P. N. (2002). Textbook on preventing drug addiction. Hanoi, Vietnam: The People Police Academy

Thomas, A. (2002). Survival of the druggies-Taking narcotics may be part of our evolutionary inheritance. New scientist, 173(2336), 11.

Thompson, G. (2007). Abstinence approaches to addiction treatment. Retrieved 02/08/2017, from International Network on Personal Meaning https://www.meaning.ca/archives/archive/art ED col may 06 abstinenceaddition-treatment $\mathrm{G}$ Thompson.htm

Thomson, N. (2013). Harm reduction history, response, and current trends in Asia. Journal of Food and Drug Analysis, 21(4), S113-S116.

Timmermans, S., \& Tavory, I. (2012). Theory construction in qualitative research: From grounded theory to abductive analysis. Sociological Theory, 30(3), 167-186.

Tkatchenko-Schmidt, E., Renton, A., Gevorgyan, R., Davydenko, L., \& Atun, R. (2008). Prevention of HIV/AIDS among injecting drug users in Russia: Opportunities and barriers to scaling-up of harm reduction programmes. Health Policy, 85(2), 162-171.

Tomori, C., Go, V. F., Huong, N. M., Binh, N. T., Zelaya, C. E., Celentano, D. D., \& Quan, V. M. (2014). "In their perception we are addicts": Social vulnerabilities and sources of support for men released from drug treatment centers in Vietnam. International Journal of Drug Policy, 25(5), 897-904.

Trace, M., Somogyi, G. L., Hallam, C., \& Bewley-Taylor, D. (2007). Report of the third Beckley international drug policy seminar. Retrieved from The Beckley Foundation Drug Policy Programme: http://reformdrugpolicy.com/wpcontent/uploads/2011/10/paper 11.pdf

Tran, B. X., Ohinmaa, A., Duong, A. T., Do, N. T., Nguyen, L. T., Mills, S., . . Jacobs, P. (2012). Cost-effectiveness of methadone maintenance treatment for HIV-positive drug users in Vietnam. AIDS Care, 24(3), 283-290.

Tran, B. X., Ohinmaa, A., Duong, A. T., Do, N. T., Nguyen, L. T., Nguyen, Q. C., ... Houston, S. (2012). Changes in drug use are associated with health-related quality of life improvements among methadone maintenance patients with HIV/AIDS. Quality of Life Research, 21(4), 613-623. doi:10.1007/s11136-011-9963-y

Trujols, J. (2015). The brain disease model of addiction: Challenging or reinforcing stigma? The Lancet Psychiatry, 2(4), 292. doi:10.1016/s2215-0366(15)00050-4

TuoitreNews. (2014). Collecting junkies helps improve crime situation in Vietnam metropolis. Retrieved 21/02/2017, from TuoitreNews 
http://tuoitrenews.vn/society/25063/collecting-junkies-helps-improve-crimesituation-in-vietnam-metropolis

TuoitreNews. (2016). Vietnam police arrest prime suspect in killings of four family members. Retrieved 21/02/2017, from TuoitreNews http://tuoitrenews.vn/society/37263/vietnam-police-arrest-suspect-in-killings-offour-family-members

Uchtenhagen, A. (2010). Heroin-assisted treatment in Switzerland: A case study in policy change. Addiction, 105(1), 29-37. doi:10.1111/j.1360-0443.2009.02741.x

UNAIDS. (2012). Joint statement: Compulsory drug detention and rehabilitation centres. Retrieved from United Nations, The Joint United Nations Programme on HIV/AIDS: http://www.who.int/hiv/topics/idu/joint statement 20120308.pdf

UNAIDS. (2016). Do not harm: Health, human rights and people who use drugs. Retrieved from Joint United Nations Programme on HIV/AIDS: http://www.unaids.org/sites/default/files/media asset/Do no harm slides.pdf

United Nations. (1961). Single convention on narcotic drugs. New York, New York, USA, United Nations: UNODC Retrieved from https://www.unodc.org/pdf/convention 1961 en.pdf.

United Nations. (1988). Convention against the illicit traffic in narcotic drugs and psychotropic substances 1988. New York, New York, USA: United Nations: UNODC Retrieved from https://www.unodc.org/unodc/en/commissions/CND/conventions.html.

UNODC. (2007). Anual report 2007. Retrieved from The United Nation Office on Drugs and Crime: https://www.unodc.org/pdf/annual report 2007/AR06 fullreport.pdf

UNODC. (2010). Country report of Vietnam: Drug situation in Vietnam in 2010. Retrieved from Vietnam, The United Nation Office on Drugs and Crime: https://www.unodc.org/pdf/vietnam/strategic programme framework vietnam.pd $\underline{f}$

UNODC. (2015). World drug report 2015. Retrieved from The United Nation Office on Drugs and Crime:

https://www.unodc.org/documents/wdr2015/World Drug Report 2015.pdf

UNODC. (2016a). Vietnam country programme. Retrieved from Vietnam, United Nations Office on Drugs and Crime: https://www.unodc.org/documents/southeastasiaandpacific/vietnam/publication/C $\underline{P \text { final E.pdf }}$ 
UNODC. (2016b). World drug report. Retrieved from Vienna, Austria, the United Nation Office on Drugs and Crime:

https://www.unodc.org/doc/wdr2016/WORLD DRUG REPORT 2016 web.pdf

UNODC. (2017). The challenge of synthetic drugs in East and South-East Asia: Trends and patterns of amphetamine-type stimulants and new psychoactive substances.

Retrieved from Global SMART Programme, United Nations Office on Drugs and Crime:

https://www.unodc.org/documents/scientific/Trends and Patterns of ATS and N PS 2017.pdf

Urquhart, C. (2012). Grounded theory for qualitative research: A practical guide. London, UK: Sage.

USAID. (2011). Understanding and challenging stigma towards injecting drug users and HIV in Vietnam - Toolkit for action. Retrieved from Hanoi, Vietnam, Institute for Social Development Studies (ISDS) and InternationalCenter for Research on Women (ICRW): http://www.icrw.org/files/publications/Toolkit for action - Reducing stigma toward drug addiction and HIV in Vietnam.PDF

VAAC. (2011). Evaluation of the epidemiological impact of harm reduction programs on HIV in Vietnam. Retrieved from Vietnam Administration for HIV/AIDS Prevention and Control (VAAC)- Ministry of Health:

http://www.aidsdatahub.org/sites/default/files/documents/Vietnam Impact Evalua tion Report 2011.pdf

Van Dorp, E. L., Yassen, A., \& Dahan, A. (2007). Naloxone treatment in opioid addiction: The risks and benefits. Expert opinion on drug safety, 6(2), 125-132.

Vietnam Steering Commitee on Smoking and Health. (2016). Hỏi và đáp: Phòng chống tác hại của thuốc lá (English translation: Questions and answers: Preventing the danger and harm of tobacco). Hanoi, Vietnam: Medical Publisher.

Vietnamese Government. (2014). Vietnam global AIDS response congress report: Following up the 2011 political declaration on HIV/AIDS. Retrieved from National Committee for AIDS, Drugs, Prostitution Prevention and Control - Vietnam:

http://www.aidsdatahub.org/Vietnam-Global-AIDS-Response-Progress-Report-2014

Volkow, N. D. (2007). Drugs, brains, and behavior: The science of addiction. Retrieved from US Department of Health and Human Services, National Institute on Drug Abuse.: https://www.nlm.nih.gov/medlineplus/magazine/issues/spring07/articles/spring07p g14-17.html

Volkow, N. D., Koob, G. F., \& McLellan, A. T. (2016). Neurobiologic advances from the brain disease model of addiction. New England Journal of Medicine, 374(4), 363-371. 
Vrecko, S. (2010). Birth of a brain disease: Science, the state and addiction neuropolitics. History of the Human Sciences, 23(4), 52-67.

VTV. (2013). Rice exports in 2012 reached a record. Retrieved 01/03/2017, from Vietnam Televison http://vtv.vn/kinh-te/xuat-khau-gao-nam-2012-dat-ky-luc-68736.htm

Vu, V. H. (2000). Chứng cứ khoa học cho nâng cao hiệu quả phòng chống tội phạm ma túy (English translation: Scientific evidence for enhancing the effectiveness of preventing and combating drug crimes). Hanoi, Vietnam: Ministry of Public Security.

Vu, V. H. (2007). Phòng chống ma túy trận chiến cáp bách của toàn xã hội (English translation: Preventing drugs - A desperate battle of the whole society. Hanoi, Vietnam: Labour Publisher.

Vuong, T. (2016). Economic evaluation comparing the cost-effectiveness of center-based compulsory rehabilitation (CCT) and voluntary community-based methadone maintenance treatment (MMT) in Hai Phong City, Vietnam. (Unpublished PhD thesis), University of New South Wales, Sydney, Australia.

Vuong, T., Ali, R., Baldwin, S., \& Mills, S. (2012). Drug policy in Vietnam: A decade of change? International Journal of Drug Policy, 23(4), 319-326.

Vuong, T., Nguyen, N., Le, G., Shanahan, M., Ali, R., \& Ritter, A. (2017). The political and scientific challenges in evaluating compulsory drug treatment centers in Southeast Asia. Harm Reduction Journal, 14(1), 2.

Vuong, T., Shanahan, M., Nguyen, N., Le, G., Ali, R., Pham, K., . . Ritter, A. (2016). Costeffectiveness of center-based compulsory rehabilitation compared to communitybased voluntary methadone maintenance treatment in Hai Phong City, Vietnam. Drug Alcohol Depend, 168, 147-155.

Walters, G. D. (1994). Drugs and crime in lifestyle perspective. London, UK: Sage.

Weatherburn, D. (2009). Dilemmas in harm minimization. Addiction, 104(3), 335-339.

Weatherburn, D., Jones, C., Snowball, L., \& Hua , J. (2008). NSW drug court: A re-evaluation of its effectiveness. Retrieved from Sydney, NSW, Bureau of Crime Statistics and Research: https://www.bocsar.nsw.gov.au/Documents/CJB/cjb121.pdf

Webster, C. (2007). Drug treatment. In M. Simpson, T. Shildrick, \& R. Macdonald (Eds.), Drugs in Britain: Supply, consumption and control (pp. 141-159). New York, New York, USA: Palgrave Macmillan. 
Werb, D., Kamarulzaman, A., Meacham, M., Rafful, C., Fischer, B., Strathdee, S., \& Wood, E. (2016). The effectiveness of compulsory drug treatment: A systematic review. International Journal of Drug Policy, 28, 1-9.

Werb, D., Rowell, G., Guyatt, G., Kerr, T., Montaner, J., \& Wood, E. (2011). Effect of drug law enforcement on drug market violence: A systematic review. International Journal of Drug Policy, 22(2), 87-94.

West, R. (2001). Theories of addiction. Addiction, 96(1), 3-13.

West, R., \& Brown, J. (2013). Theory of addiction. Chichester, West Sussex, UK: John Wiley \& Sons.

Wheeldon, J. (2010). Mapping mixed methods research: Methods, measures, and meaning. Journal of Mixed Methods Research, 4(2), 87-102.

Wheeldon, J., \& Ahlberg, M. (2012). Mapping mixed-methods research: Theories, models, and measures Visualizing social science research: Maps, methods, \& meaning (pp. 113-148). London, UK: Sage.

Wheeler, E., Davidson, P. J., Jones, T. S., \& Irwin, K. S. (2012). Community-based opioid overdose prevention programs providing naloxone-United States, 2010. MMWR. Morbidity and mortality weekly report, 61(6), 101.

White, B., Dore, G. J., Lloyd, A. R., Rawlinson, W. D., \& Maher, L. (2014). Opioid substitution therapy protects against hepatitis $C$ virus acquisition in people who inject drugs: The HITS-c study. The Medical Journal of Australia, 201, 326-329.

WHO. (2003). Neuroscience of psychoactive substance use and dependence. Retrieved from World Health Organization:

http://www.who.int/substance_abuse/publications/en/Neuroscience.pdf

WHO. (2009). Assessment of compulsory treatment of people who use drugs in Cambodia, China, Malaysia and Vietnam: An application of selected human rights principles (929061417X). Retrieved from World Health Organization:

http://www.wpro.who.int/publications/docs/FINALforWeb_Mar17_Compulsory Tre atment.pdf

Wilbanks, W. (1989). The danger in viewing addicts as victims: A critique of the disease model of addiction. Criminal Justice Policy Review, 3(4), 407-422.

Wilcox, W. (2000). In their image: The Vietnamese Communist Party, the" West" and the social evils campaign of 1996. Bulletin of Concerned Asian Scholars, 32(4), 15-24. 
Wilkins, C., Prasad, J., Parker, K., Barnes, H. M., Asiasiga, L., \& Rychert, M. (2015). New Zealand arrestee drug use monitoring (NZ-ADUM), 2010-2014. Retrieved from Shore \& Whariki Research Centre, Massey University, Wellington, New Zealand: http://www.police.govt.nz/sites/default/files/publications/2010-2014-nz-adumreport.pdf

Windle, J. (2012). The suppression of illicit opium production in Vietnam: An introductory narrative. Crime, Law and Social Change, 57(4), 425-439.

Windle, J. (2015). A slow march from social evil to harm reduction: Drugs and drug policy in Vietnam. Journal of Drug Policy Analysis, 10(2), 1-16.

Windle, J. (2016). Security trumps drug control: How securitization explains drug policy paradoxes in Thailand and Vietnam. Drugs: Education, Prevention and Policy, 23(4), 344-354.

Winkelmayer, W. C., Weinstein, M. C., Mittleman, M. A., Glynn, R. J., \& Pliskin, J. S. (2002). Health economic evaluations: The special case of end-stage renal disease treatment. Medical Decision Making, 22(5), 417-430.

Wodak, A. (1998). Health, HIV infection, human rights, and injecting drug use. Health and Human Rights, 24-41.

Wodak, A. (2011). Demand reduction and harm reduction. Retrieved from Global Commission on Drug Policies: http://www.globalcommissionondrugs.org/wpcontent/themes/gcdp v1/pdf/Global Com Alex Wodak.pdf

Wodak, A. (2014). The abject failure of drug prohibition. Journal of Criminology, 47(2), 190201.

Wood, E., Tyndall, M. W., Montaner, J. S., \& Kerr, T. (2006). Summary of findings from the evaluation of a pilot medically supervised safer injecting facility. Canadian Medical Association Journal, 175(11), 1399-1404.

Wood, W. R. (2014). Punitive populism. Retrieved 14/06/2017, from The Encyclopedia of Theoretical Criminology https://www.researchgate.net/profile/William Wood27/publication/263162318 Pu nitive Populism An Entry to the Encyclopedia of Theoretical Criminology/links/ 5a6fe84c458515015e61ea49/Punitive-Populism-An-Entry-to-the-Encyclopedia-ofTheoretical-Criminology.pdf

Wu, Z. (2013). Arguments in favour of compulsory treatment of opioid dependence. Retrieved from Bulletin of the World Health Organization, World Health Organization: 
https://search.proquest.com/docview/1321922517/fulltextPDF/92F130B33BB423AP $\underline{\mathrm{Q} / 1 \text { ? accountid }=14782}$

Yeboah, D. A. (2008). Research methodologies in criminology. New York, New York, USA: Nova Science.

Zinberg, N. E. (1986). Drug, set, and setting: The basis for controlled intoxicant use. New Haven, Connecticut, USA: Yale University Press. 


\section{Appendices}

\section{Appendix One: The Differences Between Abstinence Based and Based Harm Reduction Approaches \\ (Summarised from Chapter Two \& Three)}

\begin{tabular}{|c|c|c|}
\hline & Harm Reduction & Abstinence \\
\hline \multirow{8}{*}{$\begin{array}{l}\text { Views on } \\
\text { Drug Use }\end{array}$} & a normal human behaviour & never acceptable or permissible \\
\hline & $\begin{array}{l}\text { all drugs can be used in recreational } \\
\text { and problematic ways }\end{array}$ & $\begin{array}{l}\text { all illegal drug use is problematic and } \\
\text { all possession should be a crime }\end{array}$ \\
\hline & $\begin{array}{l}\text { some less harmful drugs can be } \\
\text { legalised }\end{array}$ & $\begin{array}{l}\text { all illegal drug use should be } \\
\text { eliminated }\end{array}$ \\
\hline & $\begin{array}{l}\text { no distinctions made between legal } \\
\text { drugs, pharmaceuticals or illicit } \\
\text { drugs. }\end{array}$ & $\begin{array}{l}\text { distinguishing between legal and } \\
\text { illegal drugs }\end{array}$ \\
\hline & $\begin{array}{l}\text { all users are different, given their } \\
\text { preferred drug use and personal } \\
\text { circumstances. They may use drugs in } \\
\text { recreational and problematic ways }\end{array}$ & all users are the same \\
\hline & $\begin{array}{l}\text { a minority of users will struggle with } \\
\text { addiction, but most users will not }\end{array}$ & $\begin{array}{l}\text { all users struggle with addiction, and } \\
\text { as such pose a threat to themselves } \\
\text { and society }\end{array}$ \\
\hline & $\begin{array}{l}\text { users are individuals with sovereign } \\
\text { rights over their body, and deserve } \\
\text { respect }\end{array}$ & $\begin{array}{l}\text { users become 'addicts' who cannot be } \\
\text { trusted. They are criminals and } \\
\text { diseased persons in need of } \\
\text { rehabilitation }\end{array}$ \\
\hline & $\begin{array}{l}\text { reasons for using illicit drugs are not } \\
\text { different to the motivations for legal } \\
\text { drugs }\end{array}$ & $\begin{array}{l}\text { people who use drugs have low moral } \\
\text { standards and make bad choices }\end{array}$ \\
\hline $\begin{array}{l}\text { Official } \\
\text { Responses }\end{array}$ & $\begin{array}{l}\text { should minimise any negative } \\
\text { consequences of drug use, as the } \\
\text { ongoing use of drugs is not an issue } \\
\text { per se }\end{array}$ & $\begin{array}{l}\text { should not accommodate ongoing } \\
\text { drug use }\end{array}$ \\
\hline
\end{tabular}




\begin{tabular}{|c|c|c|}
\hline & Harm Reduction & Abstinence \\
\hline \multirow{4}{*}{$\begin{array}{l}\text { Official } \\
\text { Responses }\end{array}$} & $\begin{array}{l}\text { people who use drugs play a central } \\
\text { role in resolving any drug problems }\end{array}$ & $\begin{array}{l}\text { clinicians play an 'expert' role in } \\
\text { resolving problems by telling users } \\
\text { what to do }\end{array}$ \\
\hline & $\begin{array}{l}\text { consumption and personal } \\
\text { possession of drugs should not be } \\
\text { policed, prosecuted or punished }\end{array}$ & $\begin{array}{l}\text { 'drug' use should be prohibited and } \\
\text { punished, to reduce supply and } \\
\text { demand }\end{array}$ \\
\hline & $\begin{array}{l}\text { any 'relapse' following interventions } \\
\text { is a natural, expected occurrence and } \\
\text { should be viewed as a learning } \\
\text { experience }\end{array}$ & $\begin{array}{l}\text { any 'relapse' following interventions is } \\
\text { a sign of individual failure to conform } \\
\text { to 'treatment' }\end{array}$ \\
\hline & $\begin{array}{l}\text { compulsory treatment threatens } \\
\text { human rights }\end{array}$ & $\begin{array}{l}\text { compulsory treatment is the only } \\
\text { option for the majority of people who } \\
\text { use drugs }\end{array}$ \\
\hline
\end{tabular}




\section{Appendix Two: Vietnamese Illicit Drug Schedules}

Because the Vietnamese Illicit Drugs Classification, which includes four schedules, is lengthy, I just provide the link to access this classification.

https://drive.google.com/file/d/1fx4qFn2MYf9o3FMEm 71 fVTA-

5YfQQV/view?usp=sharing 


\section{Appendix Three: Survey Questions}

\section{Survey questions}

\section{Demographic survey}

Direction: Please place a ' $X$ ' next to the term that best describes you

\begin{tabular}{|c|c|c|c|}
\hline 1. Where are you working now? & \multicolumn{2}{|c|}{ a Ho Chi Minh City $\quad$ a Ha Nou City } & $\square$ Another ........ \\
\hline 2. Gender: & a Male & a Female & \\
\hline 3. How old are you? & a Under 20 & $\begin{array}{l}\square 20 \text { to } 25 \text { years old } \\
\square 31 \text { to } 40 \text { years old }\end{array}$ & $\begin{array}{l}\square 26 \text { to } 30 \text { years old } \\
\square \text { Above } 40 \text { years old }\end{array}$ \\
\hline $\begin{array}{l}\text { 4. How many years have you been in } \\
\text { practice related to drug use? }\end{array}$ & $\square 3$ years $\alpha$ less & $\square 4$ to 9 years & - Over 10 years \\
\hline $\begin{array}{l}\text { 5. What is the highest qualification } \\
\text { you hold? }\end{array}$ & $\begin{array}{l}\square \text { None } \\
\square \text { Bachelor } \\
\text { ¿ Others (Please }\end{array}$ & $\begin{array}{l}\text { 口 Certificate } \\
\text { q Master } \\
\text { snecify }\end{array}$ & $\begin{array}{l}\text { 口 College degree } \\
\text { × Doctorate }\end{array}$ \\
\hline 6. I view myself professionally as: & $\begin{array}{l}\text { a Cinician } \\
\text { a Police } \\
\text { a Educator } \\
\text { a Local community } \\
\text { a Manager } \\
\text { a Others (Please }\end{array}$ & $\begin{array}{l}\text { 口 Social worker } \\
\text { a Harm reduction of } \\
\text { a Local leader } \\
\text { y } \\
\text { a Drug addiction co } \\
\text { snecify] }\end{array}$ & icer \\
\hline $\begin{array}{l}\text { 7. What is the setting in which you } \\
\text { work }\end{array}$ & $\begin{array}{l}\text { a Health sector } \\
\text { a Law enforcemen } \\
\text { a International sec } \\
\text { a Local authority }\end{array}$ & $\begin{array}{l}\text { a Social sector } \\
\text { tt sector } \\
\text { ctor }\end{array}$ & \\
\hline $\begin{array}{l}\text { 8. Have you received any professional } \\
\text { training on drug issues? }\end{array}$ & $\begin{array}{l}\text { a Yes } \\
\text { If yes please tick a } \\
\text { a Drug control } \\
\text { a Drug treatment } \\
\text { a HIV prevention } \\
\text { 口 Ofhers (please s }\end{array}$ & $\begin{array}{l}\square \text { No } \\
\text { all that apply } \\
\square \text { Drug addiction } \\
\square \text { Drug prevention } \\
\square \text { Harm reduction }\end{array}$ & \\
\hline
\end{tabular}




\section{Perceptions of Drug Use, Drug Users and Official Responses}

Direction: Please place ' $X$ ' in the space that best corresponds to your personal attitude How far do you agree/disagree with the following statements?

\begin{tabular}{|c|c|c|c|c|c|}
\hline & $\begin{array}{c}\text { Strongly } \\
\text { agree }\end{array}$ & Agree & $\begin{array}{c}\text { Not } \\
\text { decided }\end{array}$ & Disagree & $\begin{array}{l}\text { Strongly } \\
\text { Disagree }\end{array}$ \\
\hline \multicolumn{6}{|l|}{ Drug Use } \\
\hline \multicolumn{6}{|l|}{ Drug use is a public health issue } \\
\hline \multicolumn{6}{|l|}{ Drug use is a crime problem } \\
\hline \multicolumn{6}{|l|}{ People use illegal drugs for similar reasons that people use alcohol } \\
\hline \multicolumn{6}{|l|}{ Drug use is a normal human behaviour } \\
\hline \multicolumn{6}{|l|}{ Some illegal drugs are less harmful than alcohol and tobacco } \\
\hline \multicolumn{6}{|l|}{ Cannabis overdose deaths are a problem in Vietnam } \\
\hline \multicolumn{6}{|l|}{ Taking drugs will inevitably cause drug dependence } \\
\hline \multicolumn{6}{|l|}{ Drug Users } \\
\hline \multicolumn{6}{|l|}{ Most people take drugs because they have got problems } \\
\hline \multicolumn{6}{|l|}{ Drug users are suffering from a disease } \\
\hline \multicolumn{6}{|l|}{ Drug users should not be allowed in society } \\
\hline \multicolumn{6}{|l|}{ Drug users cannot be trusted } \\
\hline \multicolumn{6}{|l|}{ Drug users need to quit all drugs and stay drug free } \\
\hline \multicolumn{6}{|l|}{ Drug relapse is caused by personal failure } \\
\hline \multicolumn{6}{|l|}{ Most people who take drugs do so recreationally and rationally } \\
\hline \multicolumn{6}{|c|}{ Only a minority of people who use drugs struggle with drug dependence } \\
\hline \multicolumn{6}{|c|}{ People should be allowed to use drugs so long as they don't harm others } \\
\hline \multicolumn{6}{|l|}{ Drug relapse should be viewed as a learning experience } \\
\hline \multicolumn{6}{|c|}{ Drug users should play a central role in deciding their treatment goals } \\
\hline \multicolumn{6}{|l|}{ Official Responses } \\
\hline \multicolumn{6}{|l|}{ We should create a society free from drugs } \\
\hline \multicolumn{6}{|l|}{ We can protect society by eradicating the supply of drugs } \\
\hline \multicolumn{6}{|l|}{ We can protect society by punishing drug possession } \\
\hline \multicolumn{6}{|l|}{ Drug users need to be forced to get treatment } \\
\hline \multicolumn{6}{|l|}{ Abstaining from drugs is the only effective treatment for drug users } \\
\hline Compulsory treatment is effective in preventing drug relapse & & & & & \\
\hline
\end{tabular}




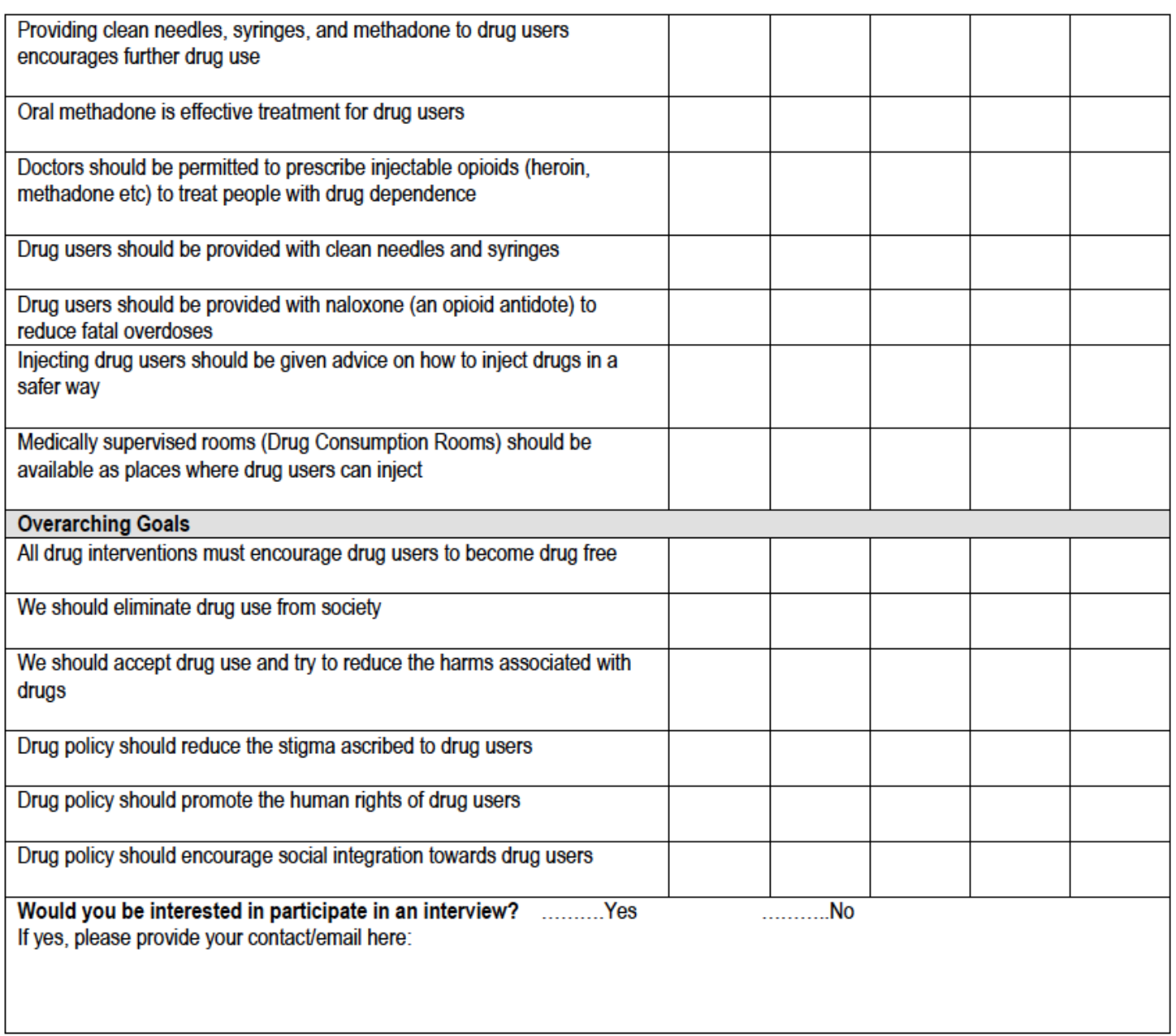

Would you like to share more thoughts about drug use, drug users or responses to drug use? If yes, please do so here: 


\section{Appendix Four: Surveyed Units}

\begin{tabular}{|c|c|c|c|}
\hline No. & Surveyed Sectors & Total & Location \\
\hline 1 & $\begin{array}{l}\text { Faculty of drug prevention and combat - Police } \\
\text { College }\end{array}$ & 13 & Ho Chi Minh City \\
\hline 2 & $\begin{array}{l}\text { Local Social Vices Prevention and Combat Unit } \\
\text { (1) }\end{array}$ & 16 & Ho Chi Minh City \\
\hline 3 & Police Stations (2) & 29 & Ho Chi Minh City \\
\hline 4 & Department of Drug Law Enforcement & 20 & Ho Chi Minh City \\
\hline 5 & Harm reduction clinics (2) & 19 & Ho Chi Minh City \\
\hline 6 & Harm Reduction Office & 13 & Ho Chi Minh City \\
\hline 7 & $\begin{array}{l}\text { The Department of Social Vices Prevention and } \\
\text { Combat - MOLISA }\end{array}$ & 22 & Ho Chi Minh City \\
\hline 8 & Drug Treatment Centre & 21 & Ho Chi Minh City \\
\hline 9 & French Expertise Organisation & 10 & Ho Chi Minh City \\
\hline 10 & $\begin{array}{l}\text { Faculty of Drug Crime Prevention - Police } \\
\text { academy }\end{array}$ & 20 & Hanoi City \\
\hline 11 & The Drug Law Enforcement Office - MPS & 19 & Hanoi City \\
\hline 12 & $\begin{array}{l}\text { Hanoi Social Vices Prevention and Combat - } \\
\text { MOLISA }\end{array}$ & 17 & Hanoi City \\
\hline 13 & Harm Reduction Office $-\mathrm{MOH}$ & 13 & Hanoi City \\
\hline 14 & UNAIDS Vietnam & 4 & Hanoi City \\
\hline 15 & Family Health International (FHI) Vietnam & 3 & Hanoi City \\
\hline 16 & UNODC Vietnam & 5 & Hanoi City \\
\hline 17 & $\begin{array}{l}\text { Substance Abuse \& Mental Health Service } \\
\text { Administration (SHAMSHA) Vietnam }\end{array}$ & 4 & Hanoi City \\
\hline 18 & $\begin{array}{l}\text { The National Committee for AIDS, Drugs, and } \\
\text { Prostitution Prevention }\end{array}$ & 1 & Hanoi City \\
\hline 19 & The Judicial Committee of Vietnam Congress & 1 & Hanoi City \\
\hline
\end{tabular}




\title{
Appendix Five: Interview Questions
}

\author{
Interview Schedule
}

Note: The nature and purposes of the research will be explained in detail before commencing interviews. All participants will be informed about their rights and that their participation is voluntary, and no names will be documented in the study. The interviews will be conducted in a private room or a negotiated place, to avoid intrusions and interference from other people. The interviews will be conducted in approximately sixty minutes. All participants can leave the interview at any time without needing to give a reason. After the interview, each participant will be given a gift for their participation.

\section{Interview questions}

1. Can you tell me about your professional background/history?

2. What is your current work? Do you work on drug issue and/or with drug users? If yes, in what way?

3. What do you see as the main drug problems in your city? And, in Vietnam?

- Has drug use changed in recent years? If so, how?

- What harms/problems emerge from drug use?

- What do you think causes or drives these harms?

4. How do you view drug use?

- Would you regard it as a crime, health or psychosocial issue ...or something else?

5. Could you tell me about people who use drugs in Vietnam?

- How would you describe them?

- What do you think about how drug users are represented in media, political or professional debate?

6. How would you describe drug addiction?

- What differences (if any) are there between drug use and drug addiction?

- Do you think that using drugs will always lead to drug addiction?

- What is the best way to treat addiction? Should it be through abstinence?

- What do you think about drug users who relapse?

- Why do you think they relapse?

- What should be done when drug users relapse?

7. What are your thoughts on the effectiveness of coercive treatment?

- In terms of approach how would you describe the approach of 06 centres?

- To what extent are 06 centres effective?

- What do you are the strengths and weaknesses of 06 centres?

8. What is your understanding of the term 'harm reduction'?

- Have you ever been trained on harm reduction?

- Can you outline some of the strengths and weaknesses of harm reduction?

- Can you provide examples on how Vietnam supports a harm reduction approach?

9. Are harm reduction strategies operated in your sphere of work? If so, how?

10. What are your thoughts on the following approaches to drugs?
a. clean needles and syringes
b. oral methadone maintenance
c. clean injectable drugs
d. drug consumption rooms
e. naloxone 
f. decriminalisation of personal possession

- Do you think they encourage ongoing drug problems, or do they minimise drug problems, how, why?

- What barriers do you think might prevent these harm reduction approaches from going ahead in Vietnam?

- What might facilitate these harm reduction approaches in Vietnam?

11. To what extent do you think drug dependency is exacerbated and sustained by struggles to do with education, employment, housing, social skills, social exclusion and human rights?

- In what way (if any) are they connected?

- To what extent do you think these issues might make it more difficult for people to move away/ or recover from drug dependency?

- What (if anything) do you think could or should be done in Vietnam to address these factors?

12. What country/countries do you think Vietnam follows in term of drug policy?

- Are there any other countries that you think Vietnam should follow in terms of drug policy? Why, what specific services/policies do you agree with, why?

13. Do you have any further comments on how Vietnam should develop drug policy, services and programmes? 


\section{Appendix Six: Ethics Approval}

MEMORANDUM

Phone $\quad 0-4-4635480$

Email susan.corbett@vuw.ac.nz

\begin{tabular}{l|l}
\hline TO & Truong Tuan Dung \\
\hline COPY TO & Julian Buchanan \\
\hline FROM & AProf Susan Corbett, Convener, Human Ethics Committee \\
\hline
\end{tabular}

\begin{tabular}{l|l}
\hline DATE & 18 December 2015 \\
\hline PAGES & 1
\end{tabular}

SUBJECT $\quad$ Ethics Approval: 22558

Perceptions of Drug Use and Drug Policy in Vietnam

Thank you for your application for ethical approval, which has now been considered by the Standing Committee of the Human Ethics Committee.

Your application has been approved from the above date and this approval continues until 1 February 2018. If your data collection is not completed by this date you should apply to the Human Ethics Committee for an extension to this approval.

Best wishes with the research.

Kind regards

Susan Corbett

Convener, Victoria University Human Ethics Committee 


\section{Appendix Seven: Consent Form}

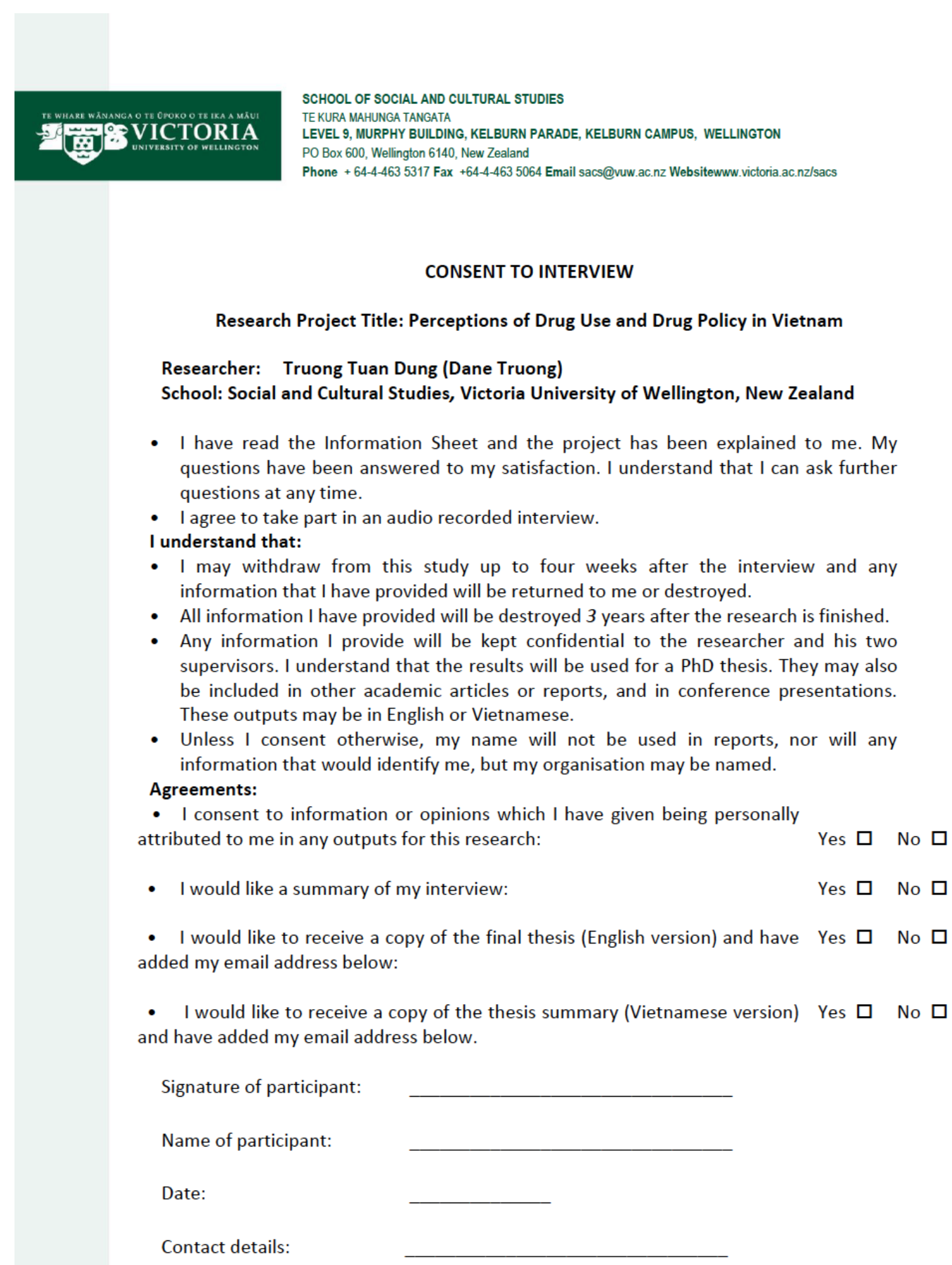




\section{Appendix Eight: Information Sheet (Interview \& Survey)}

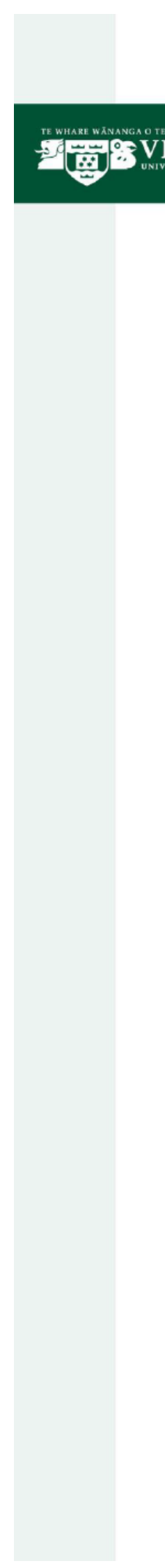

SCHOOL OF SOCIAL AND CULTURAL STUDIES

TE KURA MAHUNGA TANGATA

LEVEL 9, MURPHY BUILDING, KELBURN PARADE, KELBURN CAMPUS, WELLINGTON

PO Box 600, Wellington 6140, New Zealand

Phone + 64-4-463 5317 Fax +64-4-463 5064 Email sacs@vuw.ac.nz Websitewww.victoria.ac.nz/sacs

INFORMATION SHEET FOR INTERVIEWEES (INTERNATIONAL)

Research Project Title: Perceptions of Drug Use and Drug Policy in Vietnam

Thank you for your interest in this project. Please read this information before deciding whether or not to take part. If you decide to participate, thank you. If you decide not to take part, thank you for considering my request.

My name is Truong Tuan Dung (Dane Truong) and I am a Doctoral student in Criminology at Victoria University of Wellington, New Zealand. My thesis explores how key stakeholders perceive drug use, drug policy and drug treatment in Vietnam. Specifically, I will explore differences, conflicts, gaps, and commonalities in perceptions, laws, policies and practices. I hope it will pave the way for drug policy and practice improvements in forthcoming years in Vietnam.

This research has been approved by the Victoria University of Wellington Human Ethics Committee [provide approval number].

If you agree to take part I will interview you in a negotiated place. I will ask you questions about drug use, drug users, addiction, and responses to drug use. The interview will take approximately 60 minutes. I will digitally record the interview. You can stop the interview at any time, without giving a reason. You can withdraw from the study up to four weeks after the interview. If you withdraw, the information you provided will be destroyed.

This research is confidential. I will not name you in the final thesis or related outputs, and I will not include any information that would identify you. Your organisation may be named. Only my supervisors and I will read the notes or transcript of the interview. Some of your words may be translated into Vietnamese after I completed my study, not for the thesis, but for wider outputs. All recordings and transcripts will be kept securely and destroyed 3 years after the research ends.

The information from this research will be used in my PhD thesis. I may also use the results for conference presentations, and academic articles or reports. I will always take care not to identify you in any presentation or written work.

You do not have to accept this invitation if you do not want to. If you do decide to participate, you have the right to:

- choose not to answer any question;

- $\quad$ ask for the recorder to be turned off at any time during the interview;

- withdraw from the study up until four weeks after your interview;

- ask any questions about the study at any time;

- $\quad$ receive a copy of your interview recording (if it is recorded); 
- $\quad$ read over and comment on a written summary of your interview;

- $\quad$ agree on another name for me to use rather than your real name;

- be able to read any reports of this research by emailing the researcher to request a copy.

If you have any questions, either now or in the future, please feel free to contact either:

\section{Name: Dane Truong}

University email address:

dane.truong@vuw.ac.nz

danetruong.mps@gmail.com

\section{Name: Julian Buchanan}

Role: Primary supervisor

School: Social and Cultural Studies Victoria University of Wellington

Email: julian.buchanan@vuw.ac.nz

\section{Human Ethics Committee information}

If you have any concerns about the ethical conduct of the research you may contact the Victoria University HEC Convener: Associate Professor Susan Corbett. Email susan.corbett@vuw.ac.nz or telephone+64-4-4635480.

Thank you for your time,

\section{Dane Truong}




\section{INFORMATION SHEET FOR SURVEY PARTICIPANTS}

\section{Research Project Title: Perceptions of Drug Use and Drug Policy in Vietnam}

Thank you for your interest in this project. Please read this information before deciding whether or not to take part in my survey. If you decide to participate, thank you. If you decide not to take part, thank you for considering my request.

My name is Truong Tuan Dung (Dane Truong) and I am a Doctoral student in Criminology at Victoria University of Wellington, New Zealand.

My thesis explores how key stakeholders perceive drugs and drug policy and how they apply it in Vietnam. Specifically, it will explore differences, conflicts, gaps, and commonalities in perceptions, current laws, policies and practices. I hope that it will pave the way for drug policy and practice improvements in forthcoming years in Vietnam.

This research has been approved by the Victoria University of Wellington Human Ethics Committee [provide approval number].

If you agree to take part, you can conduct survey on paper or online through 'vuw.qualtrics.com' Website. The survey asks for your thoughts on drug use, drug users, addiction, and responses to drug use. The survey will take approximately 10 minutes. You can stop the survey at any time, without giving a reason.

This is an anonymous survey. This means that no-one will be able to identify you. My supervisors and I will read the survey results, but we will not know who provided specific answers. I will collect the survey in person if you do a survey on paper. Your survey will be returned to me in an anonymous envelope. The survey results will be kept securely and then destroyed after the research ends.

The information from this research will be used in my PhD thesis. I may also use the results for conference presentations, and other academic outputs, such as reports or journal articles.

If you have any questions, either now or in the future, please feel free to contact either:

Name: Dane Truong

University email address:

dane.truong@vuw.ac.nz

Danetruong.mps@gmail.com
Name: Julian Buchanan

Role: Primary supervisor

School: Social and Cultural Studies - Victoria University of Wellington, New Zealand

Email: julian.buchanan@vuw.ac.nz

\section{Human Ethics Committee information}

If you have any concerns about the ethical conduct of the research you may contact the Victoria University HEC Convener: Associate Professor Susan Corbett. Email susan.corbett@vuw.ac.nz or telephone +64-4-463 5480. 
Appendix Nine: Demographic Information of the Survey Participants $(n=250)$

\begin{tabular}{|c|c|c|}
\hline Characteristics & Frequency & Percent \\
\hline \multicolumn{3}{|l|}{ Location } \\
\hline Ho Chi Minh City & 182 & 72.8 \\
\hline Ha Noi City & 68 & 27.2 \\
\hline \multicolumn{3}{|l|}{ Gender } \\
\hline Male & 163 & 65.2 \\
\hline Female & 87 & 34.8 \\
\hline \multicolumn{3}{|l|}{ Ages } \\
\hline Under 20 & 0 & 0 \\
\hline $20-25$ & 7 & 2.8 \\
\hline $26-30$ & 51 & 20.4 \\
\hline $31-40$ & 106 & 42.4 \\
\hline Over 40 & 86 & 34.4 \\
\hline \multicolumn{3}{|l|}{ Work experience } \\
\hline Under 3 years & 56 & 22.4 \\
\hline 4-9 years & 93 & 37.2 \\
\hline Over 20 years & 101 & 40.4 \\
\hline \multicolumn{3}{|l|}{ Qualification } \\
\hline None & 2 & 0.8 \\
\hline Certificate & 53 & 21.2 \\
\hline College's degree & 5 & 2.0 \\
\hline Bachelor & 130 & 52.0 \\
\hline MBA & 41 & 16.4 \\
\hline PhD & 7 & 2.8 \\
\hline Others & 12 & 4.8 \\
\hline \multicolumn{3}{|l|}{ Working role } \\
\hline Clinician & 15 & 6.0 \\
\hline Social worker & 27 & 10.8 \\
\hline Police & 110 & 44 \\
\hline Harm reduction officer & 23 & 9.2 \\
\hline Local leader & 3 & 1.2 \\
\hline Educator & 3 & 1.2 \\
\hline Local community & 3 & 1.2 \\
\hline Drug addiction counsellor & 28 & 11.2 \\
\hline Manager & 37 & 11.8 \\
\hline Others & 29 & 11.6 \\
\hline
\end{tabular}




\begin{tabular}{lcc}
\hline Characteristics & Frequency & Percent \\
\hline & & \\
Work setting & 37 & \\
Health sector & 36 & 14.8 \\
Social sector & 109 & 14.4 \\
Law enforcement sector & 29 & 43.6 \\
Local authority & 24 & 11.6 \\
International sector & 15 & 9.6 \\
Others & & 6 \\
& & \\
Professional training & 205 & \\
Yes & 45 & 82 \\
No & 63 & 18 \\
Drug control & 83 & 25.2 \\
Drug addiction & 74 & 33.2 \\
Drug treatment & 99 & 29.6 \\
Drug prevention & 82 & 39.6 \\
HIV prevention & 62 & 32.8 \\
Harm reduction & 21 & 24.8 \\
Others & & 8.4 \\
& & \\
\hline
\end{tabular}




\section{Appendix Ten: The Survey Results ( $\mathrm{n}=\mathbf{2 5 0}$ )}

(Both The numbers of Survey Participants and Percentages Are Presented)

\begin{tabular}{|c|c|c|c|c|c|}
\hline & \multicolumn{5}{|c|}{ Level of Agreement: Frequency (\%) } \\
\hline & $\begin{array}{l}\text { Strongly } \\
\text { Disagree }\end{array}$ & Disagree & $\begin{array}{c}\text { Not } \\
\text { decided }\end{array}$ & Agree & $\begin{array}{c}\text { Strongly } \\
\text { Agree }\end{array}$ \\
\hline \multicolumn{6}{|l|}{ Drug Use } \\
\hline \multirow[t]{2}{*}{ Drug use is a public health issue } & 34 & 33 & 6 & 110 & 67 \\
\hline & $(13.6)$ & $(13.2)$ & $(2.4)$ & $(44)$ & $(26.8)$ \\
\hline \multirow[t]{2}{*}{ Drug use is a crime problem } & 24 & 86 & 15 & 89 & 36 \\
\hline & $(9.6)$ & $(34.4)$ & $(6.0)$ & $(35.6)$ & $(14.4)$ \\
\hline \multirow{2}{*}{$\begin{array}{l}\text { People use illegal drugs for similar reasons that people } \\
\text { use alcohol }\end{array}$} & 50 & 123 & 17 & 49 & 11 \\
\hline & (20) & $(49.2)$ & $(6.8)$ & $(19.6)$ & (4.4) \\
\hline \multirow[t]{2}{*}{ Drug use is a normal human behaviour } & 40 & 125 & 17 & 60 & 8 \\
\hline & (16) & $(50)$ & $(6.8)$ & (24) & (3.2) \\
\hline \multirow{2}{*}{$\begin{array}{l}\text { Some illegal drugs are less harmful than alcohol and } \\
\text { tobacco }\end{array}$} & 69 & 129 & 17 & 28 & 7 \\
\hline & $(27.6)$ & $(51.6)$ & $(6.8)$ & $(11.2)$ & $(2.8)$ \\
\hline \multirow[t]{2}{*}{ Cannabis overdose deaths are a problem in Vietnam } & 19 & 104 & 53 & 58 & 16 \\
\hline & $(7.6)$ & $(41.6)$ & $(21.2)$ & $(23.2)$ & (6.4) \\
\hline \multirow[t]{2}{*}{ Taking drugs will inevitably cause drug dependence } & 8 & 29 & 13 & 106 & 94 \\
\hline & $(3.2)$ & $(11.6)$ & $(5.2)$ & $(42.4)$ & $(37.6)$ \\
\hline
\end{tabular}




\section{Drug Users}

Most people take drugs because they have got

problems

Drug users are suffering from a disease

Drug users should not be allowed in society

Drug users cannot be trusted

Drug users need to quit all drugs and stay drug free

Drug relapse is caused by personal failure

Most people who take drugs do so recreationally and rationally

Only a minority of people who use drugs struggle with drug dependence

$\begin{array}{lllll}8 & 29 & 13 & 107 & 19\end{array}$

$(3.2) \quad(11.6) \quad(5.2) \quad(42.8) \quad(7.6)$

$\begin{array}{lllll}10 & 61 & 25 & 124 & 30\end{array}$

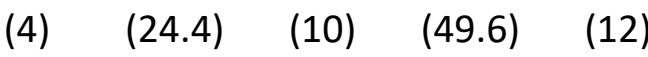

$\begin{array}{lllll}73 & 137 & 9 & 22 & 9\end{array}$

$(29.2) \quad(54.8) \quad(3.6) \quad(8.8) \quad(3.6)$

$\begin{array}{lllll}30 & 141 & 19 & 50 & 10\end{array}$

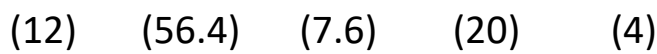

$\begin{array}{lllll}18 & 48 & 8 & 109 & 67\end{array}$

$(7.2) \quad(19.2) \quad(3.2) \quad(43.6) \quad(26.8)$

$\begin{array}{lllll}15 & 101 & 12 & 105 & 17\end{array}$

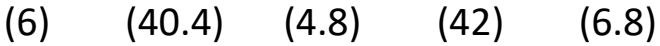

$\begin{array}{lllll}63 & 150 & 12 & 24 & 1\end{array}$

$\begin{array}{lllll}(25.2) & (60) & (4.8) & (9.6) & (0.4)\end{array}$

$\begin{array}{ccccc}35 & 126 & 14 & 67 & 8 \\ (14) & (50.4) & (5.6) & (26.8) & (3.2)\end{array}$




\begin{tabular}{l|c|c|c|c|c|}
\cline { 3 - 6 } & \multicolumn{5}{|c|}{ Level of Agreement: Frequency (\%) } \\
\cline { 3 - 6 } & $\begin{array}{c}\text { Strongly } \\
\text { Disagree }\end{array}$ & Disagree & $\begin{array}{c}\text { Not } \\
\text { decided }\end{array}$ & Agree & $\begin{array}{c}\text { Strongly } \\
\text { Agree }\end{array}$ \\
\hline People should be allowed to use drugs so long as they & 136 & 91 & 4 & 14 & 5 \\
don't harm others & $(54.4)$ & $(36.4)$ & (1.6) & (5.6) & 2
\end{tabular}

$\begin{array}{lccccccc}\text { Drug relapse should be viewed as a learning experience } & 76 & 101 & 17 & 47 & 9 \\ & (30.4) & (40.4) & (6.8) & (18.8) & (3.6) \\ & & & & & & \\ & & & & & & \\ \text { Drug users should play a central role in deciding their } & 10 & 59 & 11 & 129 & 41 \\ \text { treatment goals } & (4) & (23.6) & \text { (4.4) } & \text { (51.6) } & \text { (16.4) }\end{array}$




\begin{tabular}{|c|c|c|c|c|c|}
\hline \multicolumn{6}{|l|}{ Official Responses } \\
\hline \multirow[t]{2}{*}{ We should create a society free from drugs } & 3 & 13 & 7 & 139 & 88 \\
\hline & $(1.2)$ & $(5.2)$ & $(2.8)$ & (55.6) & (35.2) \\
\hline \multirow{2}{*}{$\begin{array}{l}\text { We can protect society by eradicating the supply of } \\
\text { drugs }\end{array}$} & 1 & 25 & 5 & 129 & 90 \\
\hline & (0.4) & (10) & (2) & (51.6) & (36) \\
\hline \multirow[t]{2}{*}{ We can protect society by punishing drug possession } & 5 & 36 & 14 & 136 & 59 \\
\hline & (2) & (14.4) & $(5.6)$ & (54.4) & (23.6) \\
\hline \multirow[t]{2}{*}{ Drug users need to be forced to get treatment } & 10 & 56 & 6 & 122 & 56 \\
\hline & (4) & (24.4) & (2.4) & $(48.8)$ & (22.4) \\
\hline \multirow{2}{*}{$\begin{array}{l}\text { Abstaining from drugs is the only effective treatment } \\
\text { for drug users }\end{array}$} & 12 & 111 & 19 & 77 & 31 \\
\hline & (4.8) & $(44.4)$ & $(7.6)$ & $(30.8)$ & (12.4) \\
\hline \multirow{2}{*}{$\begin{array}{l}\text { Compulsory treatment is effective in preventing drug } \\
\text { relapse }\end{array}$} & 10 & 93 & 30 & 98 & 19 \\
\hline & (4) & $(37.2)$ & (12) & $(39.2)$ & (7.6) \\
\hline \multirow{2}{*}{$\begin{array}{l}\text { Providing clean needles, syringes, and methadone to } \\
\text { drug users encourages further drug use }\end{array}$} & 35 & 160 & 15 & 35 & 5 \\
\hline & (14) & (64) & (6) & (14) & (2) \\
\hline \multirow[t]{2}{*}{ Oral methadone is effective treatment for drug users } & 4 & 55 & 24 & 131 & 36 \\
\hline & (1.6) & (22) & (9.6) & $(52.4)$ & $(14.4)$ \\
\hline
\end{tabular}




\begin{tabular}{l|cc|c|c|c|}
\cline { 2 - 6 } & \multicolumn{4}{c}{ Level of Agreement: Frequency (\%) } \\
\cline { 2 - 6 } & $\begin{array}{l}\text { Strongly } \\
\text { Disagree }\end{array}$ & Disagree & $\begin{array}{c}\text { Not } \\
\text { decided }\end{array}$ & Agree & $\begin{array}{c}\text { Strongly } \\
\text { Agree }\end{array}$ \\
\hline $\begin{array}{l}\text { Doctors should be permitted to prescribe injectable } \\
\text { opioids (heroin, methadone etc) to treat people with }\end{array}$ & 28 & 119 & 32 & 58 & 13 \\
$\begin{array}{l}\text { drug dependence } \\
\text { drung }\end{array}$ & $(11.2)$ & $(47.6)$ & $(12.8)$ & $(23.2)$ & (5.2)
\end{tabular}

Drug users should be provided with clean needles and syringes

$\begin{array}{ccccc}7 & 25 & 7 & 150 & 61 \\ (2.8) & (10) & (2.8) & (60) & (24.4)\end{array}$

Drug users should be provided with naloxone (an

$\begin{array}{lllll}3 & 60 & 49 & 109 & 29\end{array}$

opioid antidote) to reduce fatal overdoses

$(1.2) \quad(24) \quad(19.6) \quad(43.6) \quad(11.6)$

Injecting drug users should be given advice on how to

$\begin{array}{lllll}3 & 40 & 10 & 137 & 60\end{array}$

inject drugs in a safer way

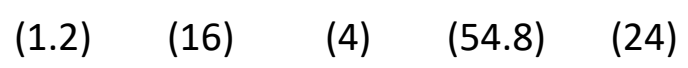

Medically supervised rooms (Drug Consumption

$\begin{array}{lllll}28 & 88 & 25 & 87 & 22\end{array}$

Rooms) should be available as places where drug users can inject

$(11.2) \quad(35.2) \quad(10) \quad(34.8) \quad(8.8)$




\section{Overarching Goals}

All drug interventions must encourage drug users to

4

7

7

8

145

86

become drug free

$(2.8)$

(3.2)

(58)

(34.4)

We should eliminate drug use from society

$\begin{array}{ccccc}5 & 9 & 12 & 124 & 100 \\ (2) & (3.6) & (4.8) & (49.6) & (40)\end{array}$

We should accept drug use and try to reduce the harms

44

$84 \quad 11$

$(17.6) \quad(33.6) \quad(4.4) \quad(31.6) \quad(12.8)$

associated with drugs

Drug policy should reduce the stigma ascribed to drug users

$\begin{array}{ccccc}4 & 16 & 13 & 171 & 46 \\ (1.6) & (6.4) & (5.2) & (68.4) & (18.4)\end{array}$

Drug policy should promote the human rights of drug

$\begin{array}{lllll}7 & 70 & 25 & 113 & 35\end{array}$
users

\begin{tabular}{|c|c|c|}
\hline (2.8 & (28) & (10) \\
\hline
\end{tabular}

Drug policy should encourage social integration towards drug users

$\begin{array}{ccccc}3 & 9 & 8 & 157 & 73 \\ (1.2) & (3.6) & (3.2) & (62.8) & (29.2)\end{array}$

